\title{
Development of a Flexible Design for Irradiation of Miniature Tensile and Charpy Test Specimens in the High Flux Isotope Reactor
}

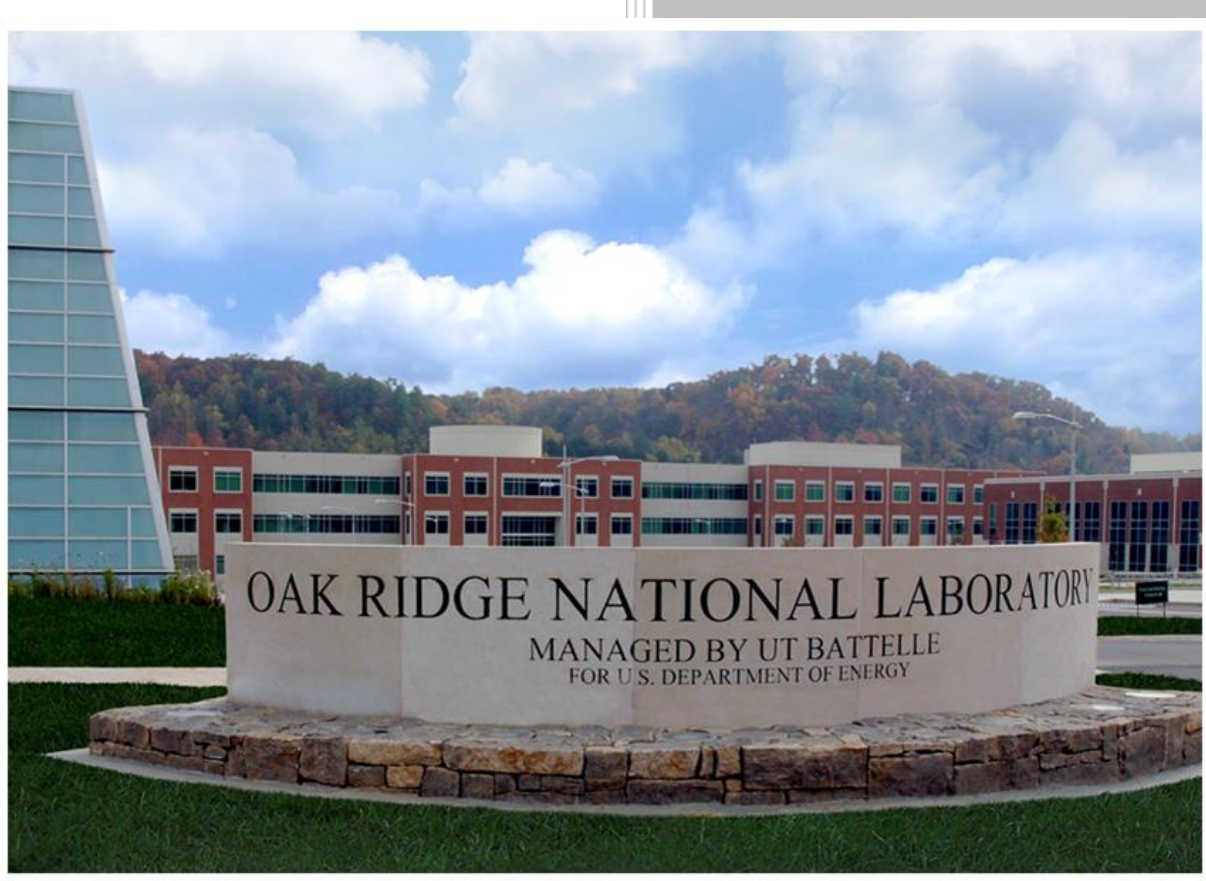

Approved for public release. Distribution is unlimited.

Richard H. Howard Kurt R. Smith

Date: July 2018 


\section{DOCUMENT AVAILABILITY}

Reports produced after January 1, 1996, are generally available free via US Department of Energy (DOE) SciTech Connect.

Website http://www.osti.gov/

Reports produced before January 1, 1996, may be purchased by members of the public from the following source:

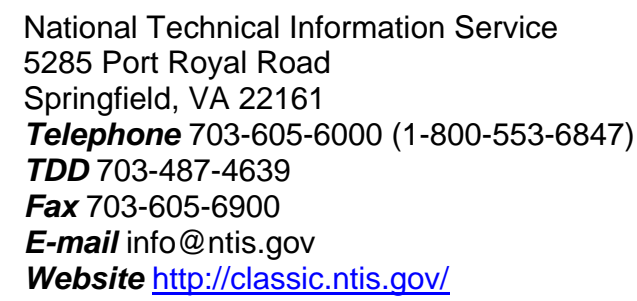

Reports are available to DOE employees, DOE contractors, Energy Technology Data Exchange representatives, and International Nuclear Information System representatives from the following source:

Office of Scientific and Technical Information

PO Box 62

Oak Ridge, TN 37831

Telephone 865-576-8401

Fax 865-576-5728

E-mail reports@osti.gov

Website http://www.osti.gov/contact.html

This report was prepared as an account of work sponsored by an agency of the United States Government. Neither the United States Government nor any agency thereof, nor any of their employees, makes any warranty, express or implied, or assumes any legal liability or responsibility for the accuracy, completeness, or usefulness of any information, apparatus, product, or process disclosed, or represents that its use would not infringe privately owned rights. Reference herein to any specific commercial product, process, or service by trade name, trademark, manufacturer, or otherwise, does not necessarily constitute or imply its endorsement, recommendation, or favoring by the United States Government or any agency thereof. The views and opinions of authors expressed herein do not necessarily state or reflect those of the United States Government or any agency thereof. 
Reactor and Nuclear Systems Division

\title{
Development of a Flexible Design for Irradiation of Miniature Tensile and Charpy Test Specimens in the High Flux Isotope Reactor
}

\author{
Richard H. Howard \\ Kurt R. Smith
}

Date Published: July 2018

Prepared by

\author{
OAK RIDGE NATIONAL LABORATORY \\ Oak Ridge, TN 37831-6283 \\ managed by \\ UT-BATTELLE, LLC \\ for the \\ US DEPARTMENT OF ENERGY \\ under contract DE-AC05-00OR22725
}





\section{CONTENTS}

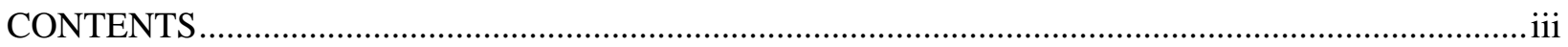

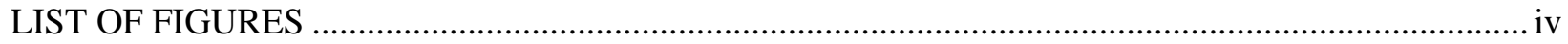

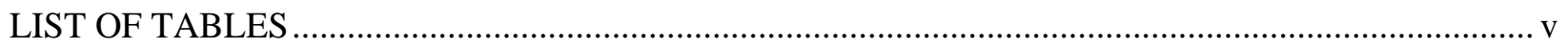

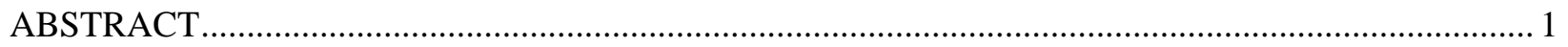

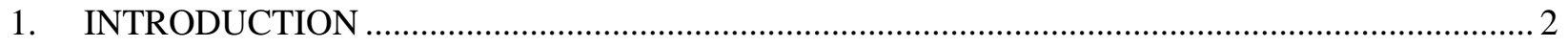

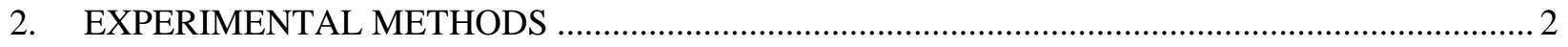

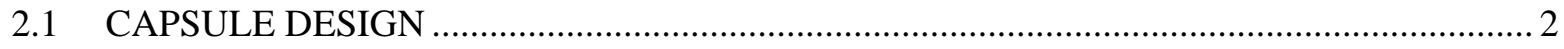

2.1.1 HFIR Irradiation and Experiment Design Concept ..................................................... 2

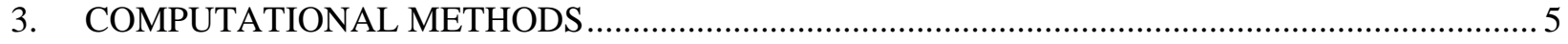

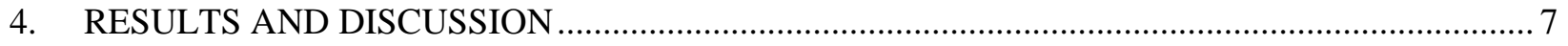

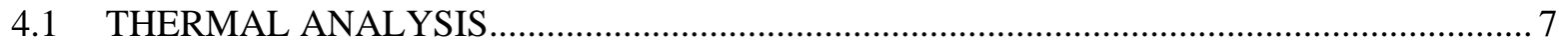

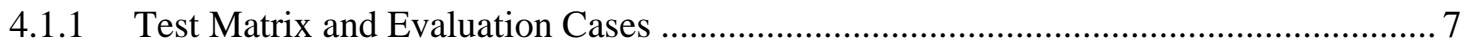

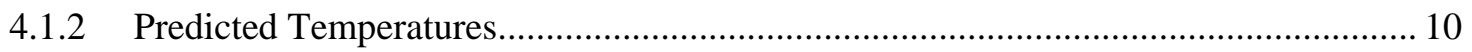

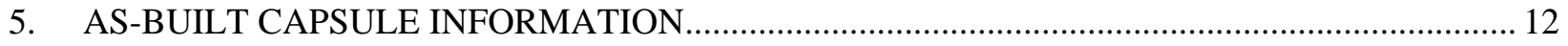

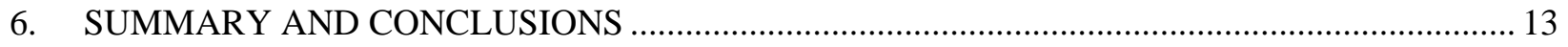

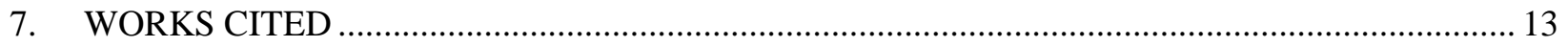

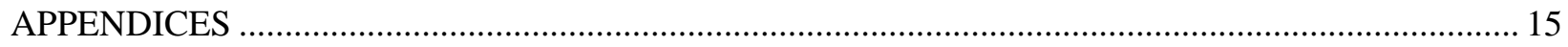

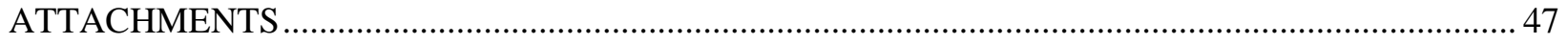




\section{LIST OF FIGURES}

Figure 1. Schematic showing transverse section view of HFIR core, reflector, and experimental positions (not to scale) [6]

Figure 2. Section view of the single-specimen-J3 (SSJ3)/MPC coupon capsule.................................... 4

Figure 3. Section view of the M4CVN bend bar capsule showing the design concept. ............................ 4

Figure 4. Meshed 3D thermal finite element model ....................................................................... 7

Figure 5. Temperature contours $\left({ }^{\circ} \mathrm{C}\right)$ for the $300^{\circ} \mathrm{C} \mathrm{SSJ} *$ case (asymmetric loading within the................ 10

Figure 6. Temperature contours for the $300^{\circ} \mathrm{C}$ bend bar case (target isotherms outlined in black)........... 11 


\section{LIST OF TABLES}

Table 1 . Summary of the experiment assembly and part detail drawings............................................. 5

Table 2. Experiment materials and material property references........................................................ 5

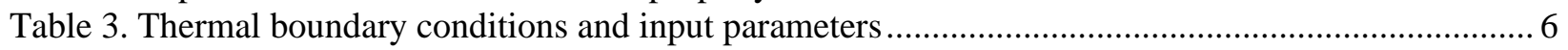

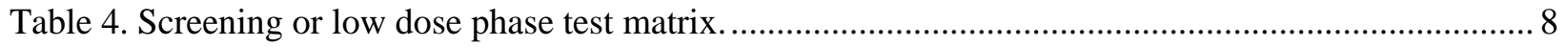

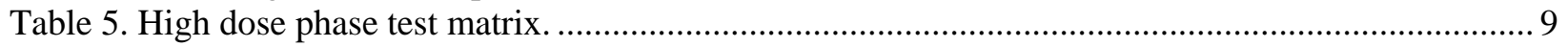

Table 6. Predicted temperatures for the tensile specimens and passive thermometry for each SSJ*/MPC specimen capsule case ........................................................................................ 11

Table 7. Predicted temperatures for the bend bar specimens and passive thermometry for each

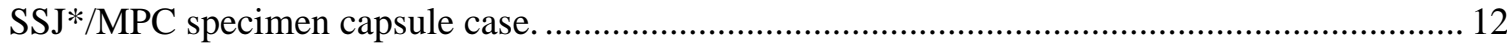




\begin{abstract}
Fusion promises a sustainable, clean, safe source of energy that could play a major role in solving the great challenge of meeting the world's growing power needs. Much of fusion technology in its current state requires further maturation to support a usable fusion reactor platform. Given that the environment in and around a sustained fusion reaction is very harsh, selecting the best materials for such a reactor is crucial. The European fusion materials community spent many years developing one such material, a lowactivation martensitic structural steel named Eurofer that is intended primarily for use in the fusion blanket modules. Although this material has reached technological maturity, nuclear performance data are required to qualify Eurofer for these applications. Karlsruhe Institute of Technology (KIT), a member of the EUROfusion consortium, has sponsored an irradiation program to establish irradiation performance data for various forms of the Eurofer alloy family. This document describes the design and development of flexible irradiation capsule designs that can be used to irradiate specimens to temperatures ranging from $\sim 200-400^{\circ} \mathrm{C}$. This report also details as-built data for the screening and high dose phases of the KIT irradiation campaign of capsules that were submitted for irradiation in the High Flux Isotope Reactor in FY18.
\end{abstract}




\section{INTRODUCTION}

Fusion promises a sustainable, clean, and safe source of energy that could play a major role in solving the great challenge of meeting the world's growing power needs. As such, much of the technology in its current state requires further maturation to support a usable fusion reactor platform. Given that the environment in and around a sustain fusion reaction is very harsh, selecting the right materials for such a reactor is crucial. The European fusion materials community spent many years developing one such material; a low activation martensitic structural steel, dubbed Eurofer, that is primarily intended for use in the fusion blanket modules [1]. Although this material has reached technological maturity, nuclear performance data is required to qualify Eurofer for such applications [2] [3]. Karlsruhe Institute of Technology (KIT), a member of the EUROfusion consortium, has sponsored an irradiation program to establish such irradiation performance data for various forms of the Eurofer alloy family. This work describes the design and development of a set of flexible irradiation capsule designs that can be used to irradiate specimens to temperatures that range from $\sim 200^{\circ} \mathrm{C}-400^{\circ} \mathrm{C}$. Furthermore, this report details "asbuilt" data for both the "screening" and "high dose" phases of the KIT irradiation campaign; capsules that were submitted for irradiation in the High Flux Isotope Reactor (HFIR) in FY18.

\section{EXPERIMENTAL METHODS}

\subsection{CAPSULE DESIGN}

\subsubsection{HFIR Irradiation and Experiment Design Concept}

\subsubsection{HFIR Irradiation}

HFIR is a beryllium-reflected, pressurized, light water-cooled and moderated flux trap-type reactor located at Oak Ridge National Laboratory (ORNL). HFIR's core consists of aluminum-clad involute-fuel plates which currently use highly enriched ${ }^{235} \mathrm{U}$ fuel to maintain steady state at a power level of $85 \mathrm{MW}$ [4]. Most of experiments are conducted in the flux trap; typically in small, uninstrumented rabbit capsules. As many as 7-9 rabbits can be stacked axially inside a single peripheral target position (PTP) holder, a target rod rabbit holder (TRRH), or the hydraulic tube (see Figure 1). The target rod and peripheral target holders have orifices that establish capsule heat transfer boundary conditions. Positions are numbered in increasing order from the bottom to the top of a PTP or TRRH. Positions TRRH-4 and PTP-5 are closest to the reactor's midplane.

The goal of this effort was to design a flexible experiment format to irradiate iron-chrome and high nickel content alloys in the flux trap. There are two general capsule formats that accept either (1) miniature single-specimen-J (SSJ2 or SSJ3) tensile specimens and other coupon-style specimens, or (2) miniature (M4CVN) Charpy or bend bar specimens. These two designs are intended for reaching temperatures between $\sim 200-400^{\circ} \mathrm{C}$ and achieve cumulative irradiation doses up to tens of displacements per atom (dpa).

Neutron and gamma radiation from HFIR fuel cause heating of experiment materials. This heating is accurately determined using neutronics models of the HFIR core and is used as an input to thermal analyses that predict component temperatures during irradiation. As mentioned above, experiments in the flux trap are almost always uninstrumented. Passive silicon carbide (SiC) temperature monitors, or thermometry, can be used to determine the irradiation temperature post irradiation [5]. However, detailed neutronic and thermal analyses are required to ensure that design temperatures are achieved. Experiment designs typically use a small insulating gas gap between the capsule internal components and external housing in contact with reactor coolant. The size of the gap and the choice of fill gas (typically helium, 
neon, or argon) inside the experiment are chosen so that the heat generated in the experimental components passes through the gas gap and gives the desired temperature drop across the gap. The temperature drop is a function of the heat flux through the gap, thermal conductivity of the fill gas, and size of the gas gap. Each of these parameters is carefully selected and modeled to achieve design temperature in an experiment.

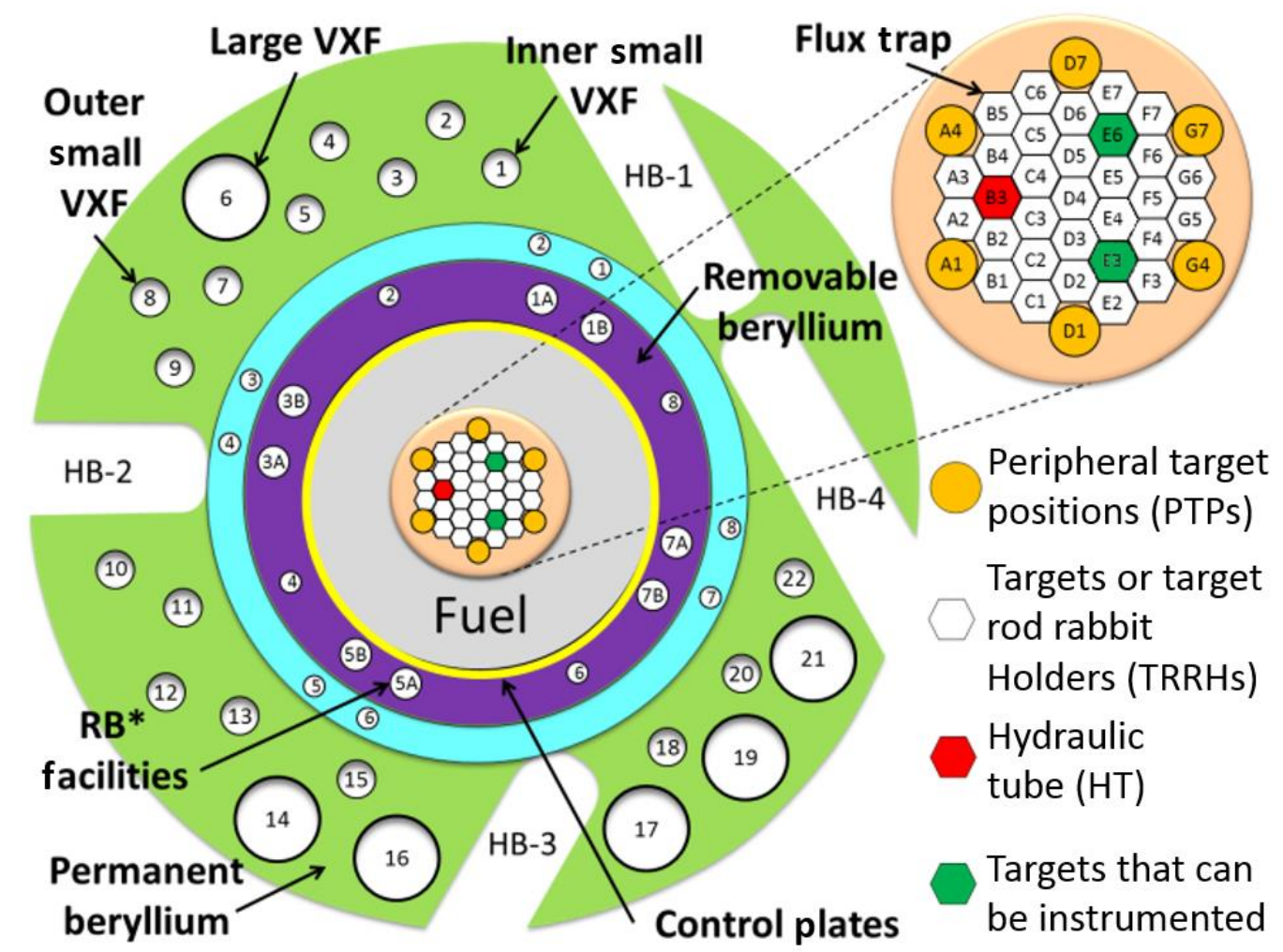

Figure 1. Schematic showing transverse section view of HFIR core, reflector, and experimental positions (not to scale) [6].

\subsubsection{Experiment Design Concept}

This report details two capsule designs (1) the miniature SSJ2 or SSJ3 (generally referred to as "SSJ*" throughout the rest of the document) tensile specimen/MPC coupon shown in Figure 2, and (2) the M4CVN bend bar shown in Figure 3. Design drawings of the experiment assembly and part details are summarized in Table 1. The outer containment for the irradiation experiment is the rabbit capsule housing, which is directly cooled on the outer surface by HFIR's primary coolant. The specimens are placed in aluminum holders with outer diameters (ODs) optimized to create gas gaps to control temperature performance. Centering tabs with a slightly larger diameter are machined into the holders to keep the assemblies centered inside the housing and to maintain a constant gas gap between the holder and the housing. Stainless steel spring pins are used in the tensile specimen design to hold the internal specimen in contact with the holder's inner walls. The chevrons are used as filler pieces that form a rectangular "coupon" shape to produce a uniform thermal load. For the bend bar design, molybdenum wires are used to ensure that the specimens remain captured in the holder. The SiC spring ensures that the specimens remain in contact with the holder wall and maintain constant heat transfer. Stainless steel liners are used to mildly insulate the F82H specimens from the high thermally conductive holder. This feature is meant to reduce thermal gradients in the specimen. Stainless steel wave springs are placed on the ends of the internal assembly between the housing and the holders to minimize axial heat loss. Grafoil insulators are used in both assemblies and are stacked on either end of the holders to reduce axial heat losses. 


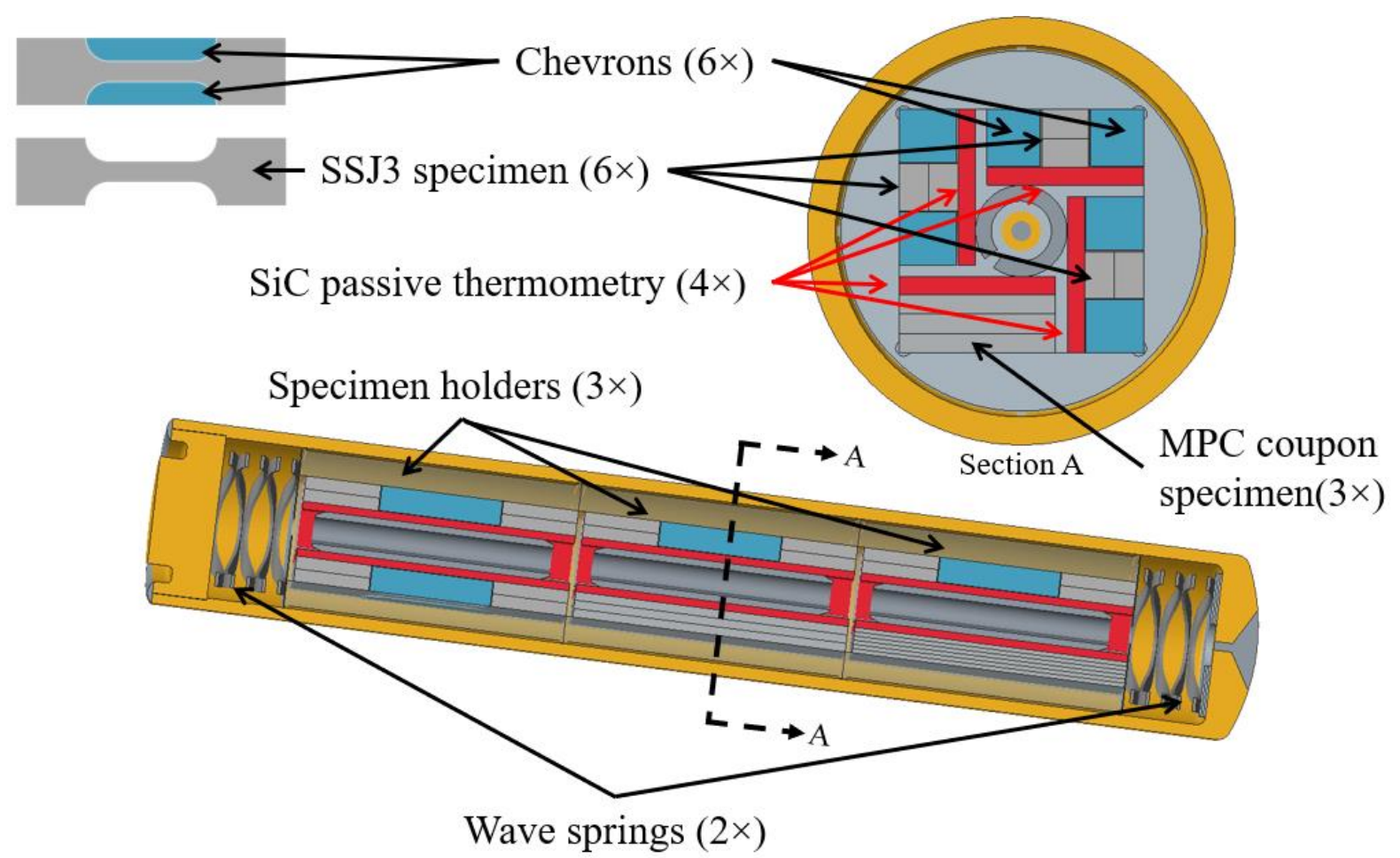

Figure 2. Section view of the single-specimen-J3 (SSJ3)/MPC coupon capsule
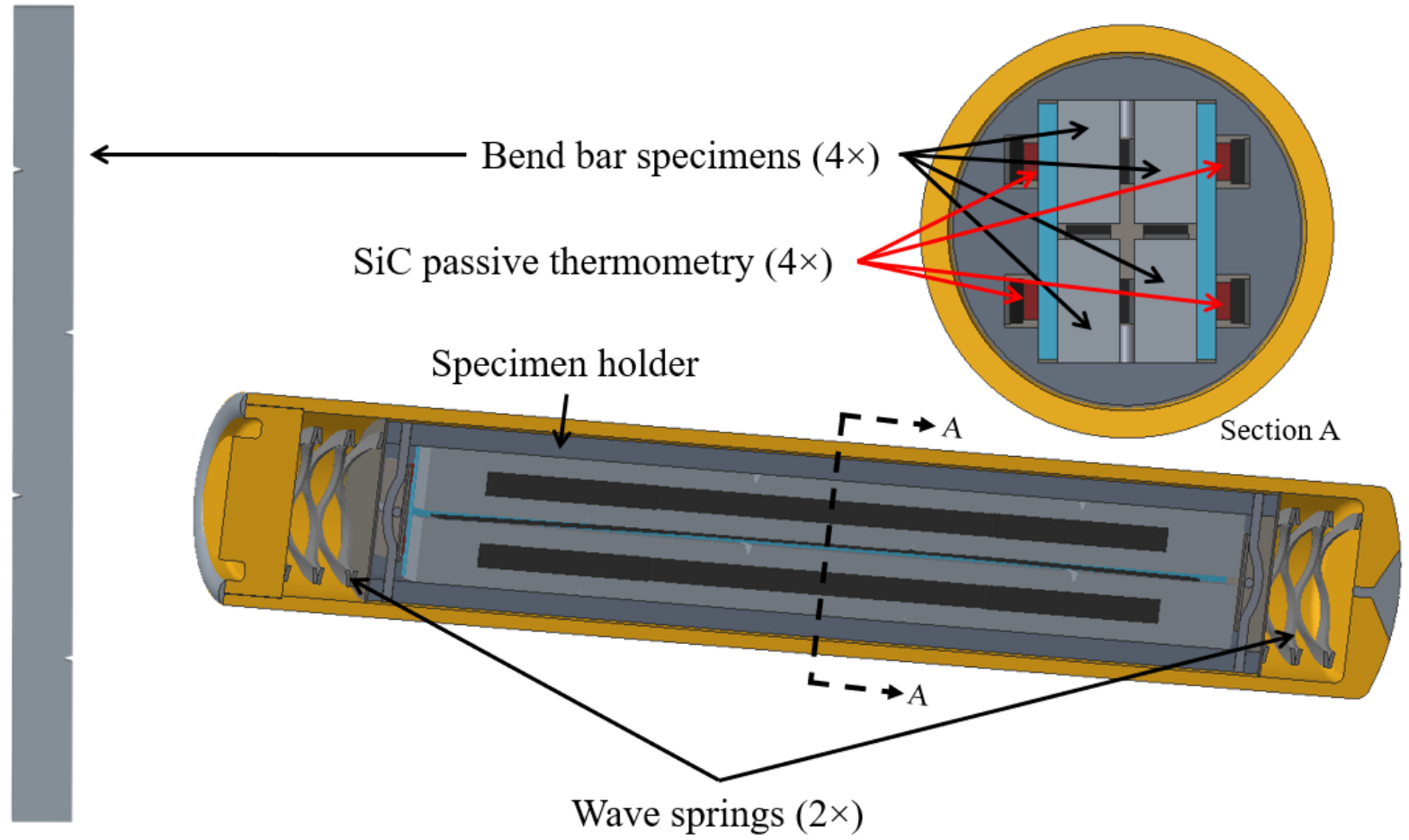

Figure 3. Section view of the M4CVN bend bar capsule showing the design concept. 
Table 1. Summary of the experiment assembly and part detail drawings

\begin{tabular}{lc}
\hline \multicolumn{1}{c}{ Drawing } & Title \\
\hline X3E020977A633, Rev. 0 & Drawings common to both designs \\
X3E020977A634, Rev. A & Target Capsule Housing Assembly [7] \\
S16-18-FUSSAM01, Rev.1 & Target Capsule Housing/End Cap Detail [8] \\
\hline \multicolumn{2}{c}{ Drawings that describe the SSJ*/MPC capsule design } \\
\hline S16-20-ESTEEL, Rev. 1 & Rabbit Capsule Assembly [10] \\
S16-21-ESTEEL, Rev. 2 & Rabbit Capsule Holder Assembly [11] \\
S16-22-ESTEEL, Rev. 1 & Rabbit Capsule Part Details [12] \\
\hline \multicolumn{2}{c}{ Drawings that describe the M4CVN capsule design } \\
\hline S16-23-ESTEELB, Rev.1 & Rabbit Capsule Assembly [13] \\
S16-24-ESTEELB, Rev.1 & Rabbit Capsule Part Details [14] \\
\hline
\end{tabular}

\section{COMPUTATIONAL METHODS}

ANSYS finite element analysis software was used to predict temperature distributions inside the experiments. These analyses use material-dependent heat generation rates (heat per unit mass), which were calculated from previously determined neutronics analyses, as inputs. Custom user-defined macros have been incorporated into ANSYS to determine thermal contact conductance between components either in contact or separated by small gas gaps that expand or contract due to thermal expansion [15]. In this way, gas gaps are not directly meshed, which significantly reduces computational time. Computer aided design (CAD) models are imported into ANSYS and are then meshed. Thermal contacts are defined to allow heat transferred between multiple bodies. Gas gap heat transfer is assumed to only include conduction, as there is very little space available for natural convection to occur. Gaps are typically on the order of microns to a few millimeters in size, and the total internal length of the capsule is less than 60 $\mathrm{mm}$. The solver accounts for thermal expansion using temperature-dependent thermal expansion data and the temperatures of contact and target surface nodes.

The ORNL Nuclear Experiments and Irradiation Testing group (formerly the Thermal Hydraulics and Irradiation Engineering group) maintains a database of design and analysis calculations (DACs) that includes temperature-dependent and some radiation dose-dependent thermophysical material properties used in thermal analyses. Properties are primarily obtained from CINDAS [16], MatWeb [17], and various literature sources. Material thermophysical properties for this calculation are included in the DACs shown in Table 2, which are available upon request.

Table 2. Experiment materials and material property references.

\begin{tabular}{lll}
\hline Part & Material & Reference \\
\hline Housing, end cap, holders & Aluminum & DAC-10-03-PROP_AL6061 [18] \\
Specimens & F82H steel & DAC-16-02-PROP_F82H [19] \\
Liners & Stainless steel & DAC-10-16-PROP_SS304 [20] \\
Insulators & Grafoil & DAC-11-16-PROP_GRAFOIL [21] \\
Thermometry & Silicon carbide & DAC-10-06-PROP_SIC(IRR) [22] \\
Wires & Molybdenum & DAC-10-11-PROP_MOLY [23] \\
Fill gas & Helium & DAC-10-02-PROP_HELIUM [24] \\
\hline
\end{tabular}

F82H thermophysical properties are used to represent the Eurofer specimen material. Mergia and Boukos show that the F82H and Eurofer 97 have significant overlap in specific heat capacity and relatively good agreement in thermal conductivity in the temperatures of interest [25]. Moreover, Klueh, et. al. show that these two version of reduced-activation ferritic/martensitic steels have virtually the same chemical compositions, with a difference of roughly $1 \%$ in chromium and some difference in the minoring alloy, 
but these two steels have similar mechanical properties [26]. As such, substituting F82H thermophysical properties for the Eurofer samples to estimate capsule thermal performance is valid for this work.

Convection boundary conditions are applied to the outer surface of the housing. Details of the calculation of the convective heat transfer coefficients and bulk coolant temperatures are summarized in DAC-11-01RAB03 [27]. These parameters were calculated using turbulent flow correlations and the axial power profile (due to neutron and gamma heat generation in the coolant) specific to target rod rabbit holders in the HFIR flux trap. The temperatures calculated in the thermal analyses are not sensitive to the convection heat transfer coefficient, as the housing surface temperatures are typically only $\sim 10^{\circ} \mathrm{C}$ warmer than the bulk coolant temperature. The heat generation rates vary in each irradiation location and as a function of axial distance from the reactor core midplane. As shown in Figure 1, there are multiple irradiation facilities designation in the HFIR flux trap. The designs discussed in this work focus on the target rod rabbit holder (TRRH) facility. Peak heat generation rates at the core midplane, parameters for determining the axial profile, and convection parameters are summarized in Table 3. Neutronics calculations performed previously for experiments with similar materials to the EUROfusion capsules [28] provided peak heat generation rates for thermal analyses, and are also reported in Table 3.

Table 3. Thermal boundary conditions and input parameters

\begin{tabular}{lc}
\hline Parameter & Value in TRRH \\
\hline Heat transfer coefficient & $47.1 \mathrm{~kW} \mathrm{~m}^{-2} \mathrm{~K}^{-1}$ \\
Bulk coolant temperature & $52^{\circ} \mathrm{C}$ \\
Peak heat generation rate for aluminum & $31.3 \mathrm{~W} / \mathrm{g}$ \\
Peak heat generation rate for F82H steel & $38.1 \mathrm{~W} / \mathrm{g}$ \\
Peak heat generation rate for Stainless Steel & $38.1 \mathrm{~W} / \mathrm{g}$ \\
Peak heat generation rate for Molybdenum & $42.0 \mathrm{~W} / \mathrm{g}$ \\
Peak heat generation rate for SiC & $31.7 \mathrm{~W} / \mathrm{g}$ \\
Peak heat generation rate for grafoil & $32.5 \mathrm{~W} / \mathrm{g}$ \\
Correlating parameter $(\sigma)$ & $30.07 \mathrm{~cm}$ \\
\hline Note: TRRH = target rod rabbit holder
\end{tabular}

The local heat generation rate for each element in the irradiation capsule is estimated with the following profile:

$$
\mathrm{q}(\text { material, } \mathrm{z})=\mathrm{q}_{\mathrm{peak}}(\text { material }) \cdot \exp \left[-\left(\frac{\mathrm{z}}{\sigma}\right)^{2}\right]
$$

where:

$\mathrm{q}=$ local heat generation rate as a function of the material and axial location

$\mathrm{q}_{\text {peak }}=$ heat generation rate at the HFIR midplane as a function of material

$\mathrm{z}=$ axial location $(\mathrm{cm})$ in the HFIR, where the midplane is at $\mathrm{z}=0$

$\sigma=$ correlating parameter $(\mathrm{cm})$

Figure 4 shows an example of a fully meshed 3D finite element model for the bend bar design. The model uses $1 / 4$ symmetry to reduce computational time. The wave springs were not modeled, and the model assumes that the ends of the holder assembly are isolated from the housing. Most of the internal components were meshed using 20-node hexagonal elements, and the model mesh size was $0.6 \mathrm{~mm}$. 


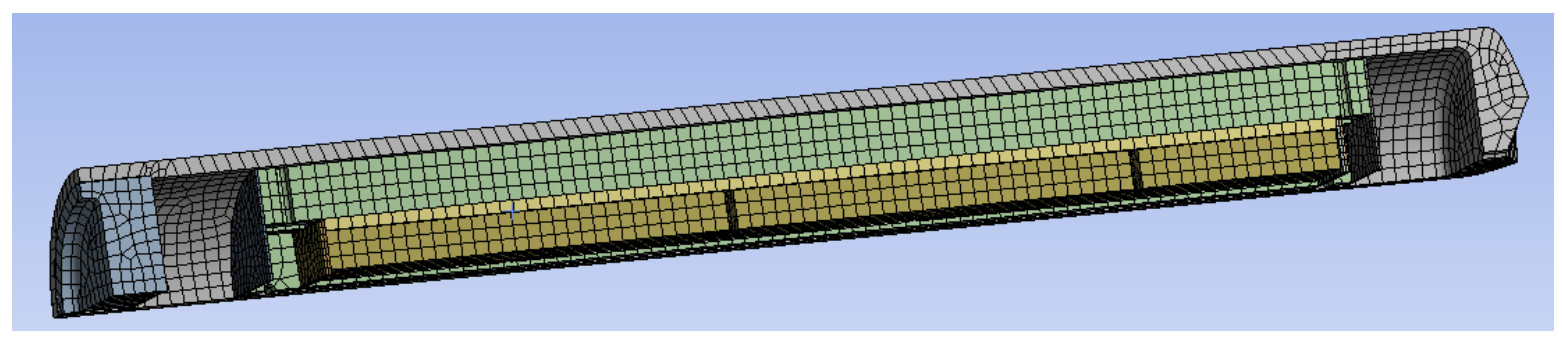

Figure 4. Meshed 3D thermal finite element model for the bend bar design with $1 / 4$ symmetry.

Similar mesh sizing was produced for the SSJ*/MPC design, but the model was radially and axially asymmetric, so these simplifications could not be made. However, the internal holder configurations are modular and are essentially equivalent. A specimen set (i.e., MPC coupons, SSJ specimen, and Eurofer chevron sets) can be considered spatially and thermally equivalent. This design flexibility exploits the similar shape footprints shared by the tensile specimens and MPC coupons. The tensile specimen temperatures are roughly equivalent to their MPC* counterparts, but bulk temperature ranges of the MPCs increase due to increased thermal contact resistances caused by stacking multiple parts. SSJ2 specimens are nominally $0.50 \mathrm{~mm}$ thick and equivalent to the MPC2 specimens, which allows for a stack of three specimens to be in a holder quadrant. Therefore, these specimen sets will have similar average temperatures and temperature spans. In contrast, the SSJ3 is $0.75 \mathrm{~mm}$ thick, and the MPC1 specimen is $0.25 \mathrm{~mm}$ thick. This allows space for 2 tensile and 6 MPC1 specimens to fit in a holder quadrant. Given that there are nearly 3 times the number of contact resistances in the MPC1 specimen stack, the average set temperature will be roughly equivalent to the SSJ counter parts, but the span of individual specimen average temperatures will be greater. It is estimated that MPC* average specimen temperature spans will range between $25-45^{\circ} \mathrm{C}$, where the SSJ* average specimen temperature spans will be $15-25^{\circ} \mathrm{C}$.

The operating temperatures of a single holder subassembly remain relatively consistent, despite specimen configuration. Optimization of the holders' outer diameters can be performed for a single subassembly configuration to establish design target temperatures. This assumption is valid because all holders are contained in a single capsule, are operating at the same temperatures, and are thermally isolated from the axial ends of the capsule. Some uncertainty on specific specimen temperatures may exist given the conditions described in the previous paragraph, but the passive thermometry will provide actual performance data for each quadrant of the as-built capsules. It is unfeasible to establish unique holder diameters for all specimen permutations that may be created from the various specimens available in this design. Therefore, using the single holder module to produce design diameters is the most practical approach to establishing design diameters.

\section{RESULTS AND DISCUSSION}

\subsection{THERMAL ANALYSIS}

\subsubsection{Test Matrix and Evaluation Cases}

Thermal analyses were performed for each capsule configuration requested by the program sponsor to target specific design temperatures (see Table 4 and Table 5). These analyses determine temperature distributions within the experiment using nominal housing and specimen dimensions and the desired fill gas, helium, to produce a specific gas gap to target an average design temperature. The primary sensitivity parameter is the gas gap itself, given that the thermophysical properties and heat generation rates of the solid materials are not greatly affected by small perturbations in temperature. Therefore, once a gas gap is established, the housing's inner diameter and corresponding holder diameter can deviate somewhat 
from the nominal dimension as long as the target gas gap is maintained. All capsule configurations were designed for positions TRRH 3 and TRRH 5 in the HFIR flux trap to ensure most favorable conditions with the widest availability. The TRRH $3 / 5$ positions are equivalent to the peripheral target position PTP 4 irradiation site. These positions are symmetrically centered around the TRRH center position, which provides the second flattest power profile (91-99\% centerline power over the length of the position) and have twice the number of available locations (32) compared to TRRH center positions.

Table 4. Screening or low dose phase test matrix.

\begin{tabular}{|c|c|c|c|c|c|c|c|}
\hline $\begin{array}{c}\text { Capsule } \\
\text { ID }\end{array}$ & Configuration & $\begin{array}{c}\text { Target } \\
\text { irradiation } \\
\text { condition }\end{array}$ & $\begin{array}{c}\text { Specimen } \\
\text { dimensions } \\
(\text { Length } \times \text { width } \times \\
\text { thickness }) \text { mm }\end{array}$ & Qty. & Material & $\begin{array}{c}\text { Pre- } \\
\text { irradiation } \\
\text { tests }\end{array}$ & $\begin{array}{c}\text { Post-irradiation } \\
\text { tests }\end{array}$ \\
\hline ES21 & $\begin{array}{l}20 \times \mathrm{SS}-\mathrm{J} 3 \\
10 \times \mathrm{MPC} 1\end{array}$ & $\begin{array}{c}2.5 \pm .38 \mathrm{dpa} \\
285^{\circ} \mathrm{C} \pm 20\end{array}$ & $\begin{array}{l}16 \times 4 \times .75 \\
16 \times 4 \times .25\end{array}$ & $\begin{array}{l}2 \text { ea. } \\
1 \text { ea. }\end{array}$ & $\begin{array}{l}10 \text { alloy } \\
\text { variants }\end{array}$ & $\begin{array}{l}\text { Photography, } \\
\text { metrology }\end{array}$ & $\begin{array}{c}\text { Vickers hardness, } \\
\text { tensile test, } \\
\text { TEM* }\end{array}$ \\
\hline ES22 & $\begin{array}{l}20 \times \mathrm{SS}-\mathrm{J} 3 \\
10 \times \mathrm{MPC} 1\end{array}$ & $\begin{array}{c}2.5 \pm .38 \mathrm{dpa} \\
315^{\circ} \mathrm{C} \pm 20\end{array}$ & $\begin{array}{l}16 \times 4 \times .75 \\
16 \times 4 \times .25\end{array}$ & $\begin{array}{l}2 \text { ea. } \\
1 \text { ea. }\end{array}$ & $\begin{array}{l}10 \text { alloy } \\
\text { variants }\end{array}$ & $\begin{array}{l}\text { Photography, } \\
\text { metrology }\end{array}$ & $\begin{array}{l}\text { Vickers hardness, } \\
\text { tensile test, TEM }\end{array}$ \\
\hline ES31 & $4 \times \mathrm{M} 4 \mathrm{CVN}$ & $\begin{array}{c}2.5 \pm .38 \mathrm{dpa} \\
300^{\circ} \mathrm{C} \pm 20\end{array}$ & $45 \times 3.3 \times 1.65$ & 2 ea. & $\begin{array}{c}2 \text { alloy } \\
\text { variants }\end{array}$ & $\begin{array}{l}\text { Pre-cracking, } \\
\text { photography, } \\
\text { metrology }\end{array}$ & $\begin{array}{c}\text { Vickers hardness, } \\
\text { fracture } \\
\text { toughness }\end{array}$ \\
\hline ES32 & $4 \times \mathrm{M} 4 \mathrm{CVN}$ & $\begin{array}{c}2.5 \pm .38 \mathrm{dpa} \\
300^{\circ} \mathrm{C} \pm 20\end{array}$ & $45 \times 3.3 \times 1.65$ & 2 ea. & $\begin{array}{c}2 \text { alloy } \\
\text { variants }\end{array}$ & $\begin{array}{l}\text { Pre-cracking, } \\
\text { photography, } \\
\text { metrology }\end{array}$ & $\begin{array}{c}\text { Vickers hardness, } \\
\text { fracture } \\
\text { toughness }\end{array}$ \\
\hline ES33 & $4 \times \mathrm{M} 4 \mathrm{CVN}$ & $\begin{array}{c}2.5 \pm .38 \mathrm{dpa} \\
300^{\circ} \mathrm{C} \pm 20\end{array}$ & $45 \times 3.3 \times 1.65$ & 2 ea. & $\begin{array}{c}2 \text { alloy } \\
\text { variants }\end{array}$ & $\begin{array}{l}\text { Pre-cracking, } \\
\text { photography, } \\
\text { metrology }\end{array}$ & $\begin{array}{c}\text { Vickers hardness, } \\
\text { fracture } \\
\text { toughness }\end{array}$ \\
\hline ES34 & $4 \times \mathrm{M} 4 \mathrm{CVN}$ & $\begin{array}{c}2.5 \pm .38 \mathrm{dpa} \\
300^{\circ} \mathrm{C} \pm 20\end{array}$ & $45 \times 3.3 \times 1.65$ & 2 ea. & $\begin{array}{c}2 \text { alloy } \\
\text { variants }\end{array}$ & $\begin{array}{l}\text { Pre-cracking, } \\
\text { photography, } \\
\text { metrology }\end{array}$ & $\begin{array}{c}\text { Vickers hardness, } \\
\text { fracture } \\
\text { toughness }\end{array}$ \\
\hline ES35 & $4 \times \mathrm{M} 4 \mathrm{CVN}$ & $\begin{array}{c}2.5 \pm .38 \mathrm{dpa} \\
300^{\circ} \mathrm{C} \pm 20\end{array}$ & $45 \times 3.3 \times 1.65$ & 2 ea. & $\begin{array}{c}2 \text { alloy } \\
\text { variants }\end{array}$ & $\begin{array}{c}\text { Pre-cracking, } \\
\text { photography, } \\
\text { metrology }\end{array}$ & $\begin{array}{c}\text { Vickers hardness, } \\
\text { fracture } \\
\text { toughness }\end{array}$ \\
\hline
\end{tabular}


Table 5. High dose phase test matrix.

\begin{tabular}{|c|c|c|c|c|c|}
\hline Capsule ID & Configuration & $\begin{array}{c}\text { Target irradiation } \\
\text { condition }\end{array}$ & $\begin{array}{c}\text { Specimen dimensions } \\
(\text { Length } \times \text { width } \times \text { thickness }) \mathrm{mm}\end{array}$ & Qty. & Pre-irradiation tests \\
\hline ES01 & $\begin{array}{c}12 \times \mathrm{SS}-\mathrm{J} 3 ; \\
6 \times \mathrm{MPC} 1 ; 6 \times \mathrm{MPC} 2\end{array}$ & $\begin{array}{c}20 \pm 2 \mathrm{dpa} \\
220^{\circ} \mathrm{C} \pm 20\end{array}$ & $\begin{array}{c}16 \times 4 \times 0.75 \\
16 \times 4 \times 0.25,16 \times 4 \times 0.5\end{array}$ & $\begin{array}{c}12 \\
6,6\end{array}$ & Photography, metrology \\
\hline ESO2 & $\begin{array}{c}12 \times \mathrm{SS}-\mathrm{J} 3 ; \\
6 \times \mathrm{MPC} 1 ; 6 \times \mathrm{MPC} 2\end{array}$ & $\begin{array}{c}20 \pm 2 \mathrm{dpa} \\
240^{\circ} \mathrm{C} \pm 20\end{array}$ & $\begin{array}{c}16 \times 4 \times 0.75 \\
16 \times 4 \times 0.25,16 \times 4 \times 0.5\end{array}$ & $\begin{array}{c}12 \\
6,6\end{array}$ & Photography, metrology \\
\hline ES03 & $\begin{array}{c}12 \times \mathrm{SS}-\mathrm{J} 3 ; \\
6 \times \mathrm{MPC} 1 ; 6 \times \mathrm{MPC} 2\end{array}$ & $\begin{array}{c}20 \pm 2 \mathrm{dpa} \\
275^{\circ} \mathrm{C} \pm 20\end{array}$ & $\begin{array}{c}16 \times 4 \times 0.75 \\
16 \times 4 \times 0.25,16 \times 4 \times 0.5\end{array}$ & $\begin{array}{c}12 \\
6,6\end{array}$ & Photography, metrology \\
\hline ES04 & $\begin{array}{c}12 \times \mathrm{SS}-\mathrm{J} 3 ; \\
6 \times \mathrm{MPC} 1 ; 6 \times \mathrm{MPC} 2\end{array}$ & $\begin{array}{c}20 \pm 2 \mathrm{dpa} \\
300^{\circ} \mathrm{C} \pm 20\end{array}$ & $\begin{array}{c}16 \times 4 \times 0.75 \\
16 \times 4 \times 0.25,16 \times 4 \times 0.5\end{array}$ & $\begin{array}{c}12 \\
6,6\end{array}$ & Photography, metrology \\
\hline ES05 & $\begin{array}{c}12 \times \mathrm{SS}-\mathrm{J} 3 \\
6 \times \mathrm{MPC} 1 ; 6 \times \mathrm{MPC} 2\end{array}$ & $\begin{array}{c}20 \pm 2 \text { dpa } \\
325^{\circ} \mathrm{C} \pm 20\end{array}$ & $\begin{array}{c}16 \times 4 \times 0.75 \\
16 \times 4 \times 0.25,16 \times 4 \times 0.5\end{array}$ & $\begin{array}{l}12 \\
6,6\end{array}$ & Photography, metrology \\
\hline ES06 & $\begin{array}{c}12 \times \mathrm{SS}-\mathrm{J} 3 ; \\
6 \times \mathrm{MPC} 1 ; 6 \times \mathrm{MPC} 2\end{array}$ & $\begin{array}{l}20 \pm 2 \mathrm{dpa} \\
350^{\circ} \mathrm{C} \pm 20\end{array}$ & $\begin{array}{c}16 \times 4 \times .75 \\
16 \times 4 \times .25,16 \times 4 \times .5\end{array}$ & $\begin{array}{l}12 \\
6,6\end{array}$ & Photography, metrology \\
\hline ES07 & $\begin{array}{c}12 \times \mathrm{SS}-\mathrm{J} 3 ; \\
6 \times \mathrm{MPC} 1 ; 6 \times \mathrm{MPC} 2\end{array}$ & $\begin{array}{c}20 \pm 2 \mathrm{dpa} \\
375^{\circ} \mathrm{C} \pm 20\end{array}$ & $\begin{array}{c}16 \times 4 \times 0.75 \\
16 \times 4 \times 0.25,16 \times 4 \times 0.5\end{array}$ & $\begin{array}{c}12 \\
6,6\end{array}$ & Photography, metrology \\
\hline ES11 & $4 \times \mathrm{M} 4 \mathrm{CVN}$ & $\begin{array}{c}20 \pm 2 \mathrm{dpa} \\
220^{\circ} \mathrm{C} \pm 20\end{array}$ & $45 \times 3.3 \times 1.65$ & 4 & $\begin{array}{l}\text { Pre-cracking, photography } \\
\text { metrology }\end{array}$ \\
\hline ES12 & $4 \times \mathrm{M} 4 \mathrm{CVN}$ & $\begin{array}{c}20 \pm 2 \mathrm{dpa} \\
240^{\circ} \mathrm{C} \pm 20\end{array}$ & $45 \times 3.3 \times 1.65$ & 4 & $\begin{array}{l}\text { Pre-cracking, photography } \\
\text { metrology }\end{array}$ \\
\hline ES13 & $4 \times \mathrm{M} 4 \mathrm{CVN}$ & $\begin{array}{c}20 \pm 2 \mathrm{dpa} \\
275^{\circ} \mathrm{C} \pm 20\end{array}$ & $45 \times 3.3 \times 1.65$ & 4 & $\begin{array}{l}\text { Pre-cracking, photography } \\
\text { metrology }\end{array}$ \\
\hline ES14 & $4 \times \mathrm{M} 4 \mathrm{CVN}$ & $\begin{array}{c}20 \pm 2 \mathrm{dpa} \\
300^{\circ} \mathrm{C} \pm 20\end{array}$ & $45 \times 3.3 \times 1.65$ & 4 & $\begin{array}{c}\text { Pre-cracking, photography } \\
\text { metrology }\end{array}$ \\
\hline ES15 & $4 \times \mathrm{M} 4 \mathrm{CVN}$ & $\begin{array}{c}20 \pm 2 \mathrm{dpa} \\
325^{\circ} \mathrm{C} \pm 20\end{array}$ & $45 \times 3.3 \times 1.65$ & 4 & $\begin{array}{c}\text { Pre-cracking, photography } \\
\text { metrology }\end{array}$ \\
\hline ES16 & $4 \times \mathrm{M} 4 \mathrm{CVN}$ & $\begin{array}{l}20 \pm 2 \mathrm{dpa} \\
350^{\circ} \mathrm{C} \pm 20\end{array}$ & $45 \times 3.3 \times 1.65$ & 4 & $\begin{array}{l}\text { Pre-cracking, photography } \\
\text { metrology }\end{array}$ \\
\hline ES17 & $4 \times \mathrm{M} 4 \mathrm{CVN}$ & $\begin{array}{c}20 \pm 2 \mathrm{dpa} \\
375^{\circ} \mathrm{C} \pm 20\end{array}$ & $45 \times 3.3 \times 1.65$ & 4 & $\begin{array}{l}\text { Pre-cracking, photography } \\
\text { metrology }\end{array}$ \\
\hline
\end{tabular}




\subsubsection{Predicted Temperatures}

Figure 5 and Figure 6 show examples of temperature contours for both styles of the rabbit assemblies. The SSJ*/MPC specimen design used the gauge length temperatures of the tensile specimens as the figures of merit. A difference in contour color can be seen between the inner and outer specimens, which is a graphical representation of the contact resistance issue described earlier in Section 3. Otherwise, the calculated temperature of this portion of the specimens is more uniform. There is a slight gradient over the widths and depths of the gauges (observed from left to right and in and out of the page), which comes from the asymmetric loading of the specimens within the holder, as shown in the upper right-hand corner of the figure. This could be corrected by centering the specimens within the cutout, but that would lead to a reduction in the number of specimens loaded into this configuration. Moreover, the gradients are small and generally within the uncertainty of the calculations based on the uncertainties of the heat generation rates, convective heat transfer coefficient, and geometric variations of machined parts.

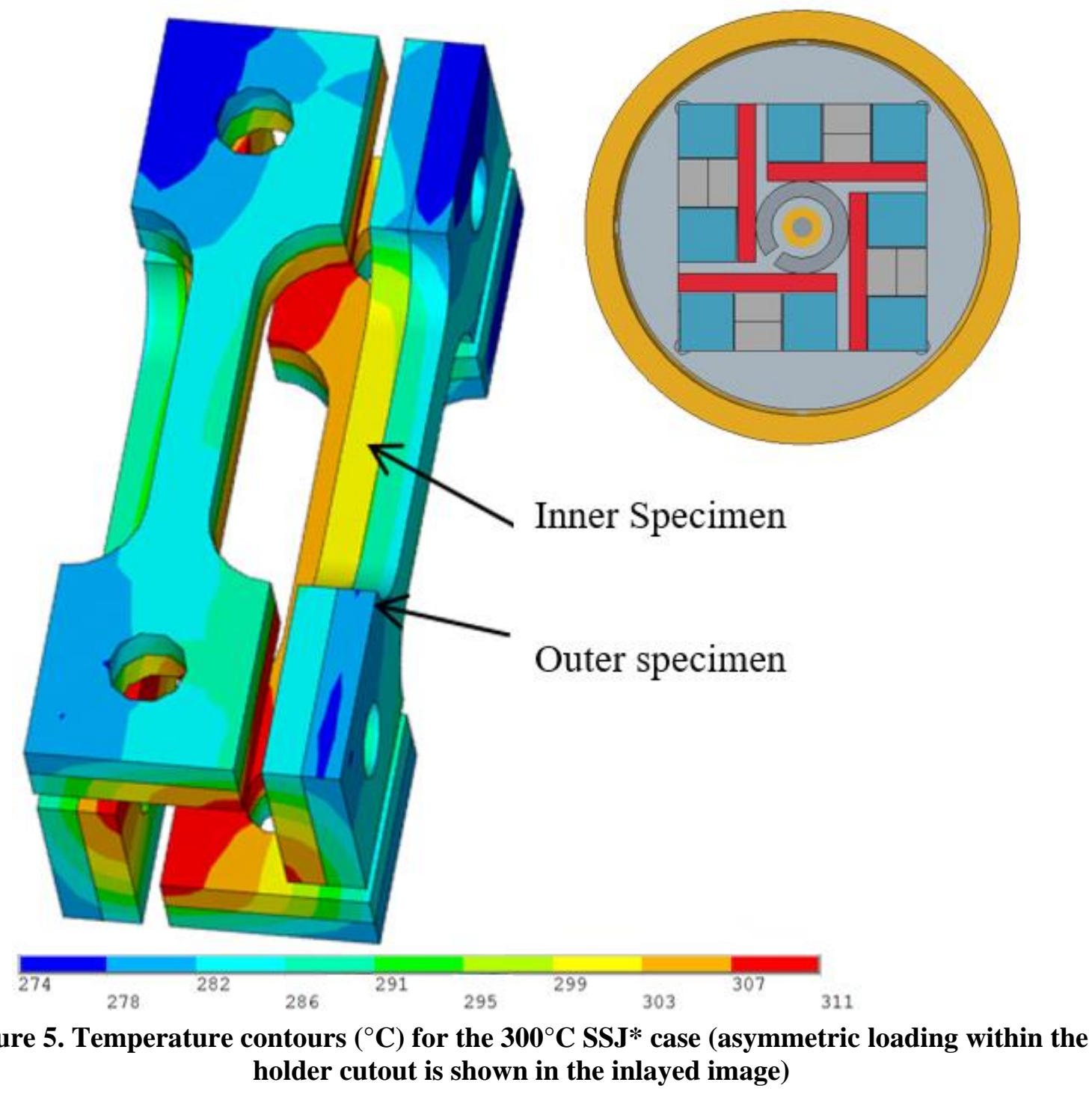



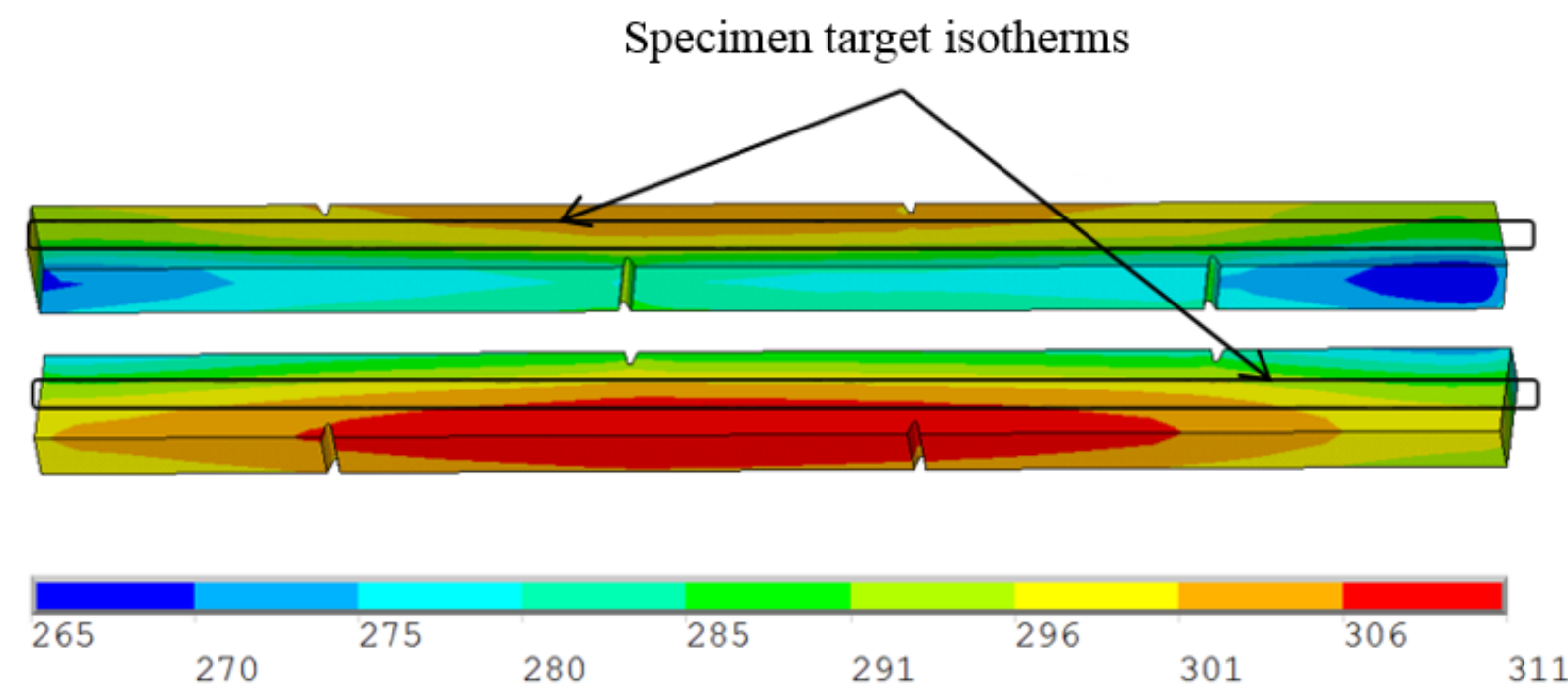

Figure 6. Temperature contours for the $300^{\circ} \mathrm{C}$ bend bar case (target isotherms outlined in black).

The average temperatures of the central volume located between the bend bar notches were used for the figure of merit for this design configuration (see outlined area in Figure 6). Given the nature of the Charpy test, crack propagation through this region dictates the performance of the specimen, so it was reasonable to select this region for use in identifying the design temperature. Moreover, it is a more central region of the specimen as shown by the reduced magnitude of thermal gradients shown in the thermal contour plots. Note that this calculation employed $90^{\circ}$ symmetry, so Figure 6 shows the four lateral sides for a single parallelepiped shape.

Table 6 summarizes average, minimum, and maximum temperatures for the specimens and thermometry of each SSJ style capsule configuration. Likewise, Table 7 summarizes average, minimum, and maximum temperatures for the M4CVN bend bar case. Thermometry data reported in these tables will be compared to temperatures determined post-irradiation using dilatometry on SiC thermometry specimens. More details are provided in the full ANSYS output files in APPENDIX A. Average specimen temperatures fall within the allowable ranges in Table 4 and Table 5. Given the high heat generation rates produced by HFIR and the proximity to the reactor's centerline, it can be problematic to reach lower temperatures. Therefore, the lower temperature cases $\left(200--240^{\circ} \mathrm{C}\right)$ were established on best effort.

Table 6. Predicted temperatures for the tensile specimens and passive thermometry for each SSJ*/MPC specimen capsule case

\begin{tabular}{|c|c|c|c|c|c|c|c|}
\hline \multirow{2}{*}{$\begin{array}{l}\text { Design diameter } \\
(\mathbf{m m})\end{array}$} & \multirow[b]{2}{*}{ Part name } & \multirow[b]{2}{*}{ Material } & \multirow[b]{2}{*}{ Fill gas } & \multirow{2}{*}{$\begin{array}{l}\text { Irradiation } \\
\text { location }\end{array}$} & \multicolumn{3}{|c|}{ Temperature $\left({ }^{\circ} \mathbf{C}\right)$} \\
\hline & & & & & $\begin{array}{c}\text { Average } \\
\left({ }^{\circ} \mathbf{C}\right)\end{array}$ & Minimum & Maximum \\
\hline \multirow{3}{*}{$\begin{array}{c}9.40 \\
\left(200^{\circ} \mathrm{C}\right)\end{array}$} & Inner SSJ tensile & Eurofer & \multirow{9}{*}{ Helium } & \multirow{9}{*}{ TRRH $3 / 5$} & 200 & 179 & 212 \\
\hline & Outer SSJ tensile & Eurofer & & & 186 & 174 & 195 \\
\hline & Thermometry & $\mathrm{SiC}$ & & & 205 & 169 & 230 \\
\hline \multirow{3}{*}{$\begin{array}{c}9.38 \\
\left(220^{\circ} \mathrm{C}\right)\end{array}$} & Inner SSJ tensile & Eurofer & & & 214 & 193 & 226 \\
\hline & Outer SSJ tensile & Eurofer & & & 200 & 188 & 208 \\
\hline & Thermometry & $\mathrm{SiC}$ & & & 219 & 184 & 244 \\
\hline \multirow{3}{*}{$\begin{array}{c}9.34 \\
\left(240^{\circ} \mathrm{C}\right)\end{array}$} & Inner SSJ tensile & Eurofer & & & 241 & 220 & 253 \\
\hline & Outer SSJ tensile & Eurofer & & & 227 & 215 & 236 \\
\hline & Thermometry & $\mathrm{SiC}$ & & & 246 & 210 & 271 \\
\hline
\end{tabular}




\begin{tabular}{|c|c|c|c|c|c|c|c|}
\hline \multirow{2}{*}{$\begin{array}{l}\text { Design diameter } \\
(\mathbf{m m})\end{array}$} & \multirow[b]{2}{*}{ Part name } & \multirow[b]{2}{*}{ Material } & \multirow[b]{2}{*}{ Fill gas } & \multirow{2}{*}{$\begin{array}{l}\text { Irradiation } \\
\text { location }\end{array}$} & \multicolumn{3}{|c|}{ Temperature $\left({ }^{\circ} \mathrm{C}\right)$} \\
\hline & & & & & $\begin{array}{c}\text { Average } \\
\left({ }^{\circ} \mathbf{C}\right)\end{array}$ & Minimum & Maximum \\
\hline \multirow{3}{*}{$\begin{array}{c}9.30 \\
\left(275^{\circ} \mathrm{C}\right)\end{array}$} & Inner SSJ tensile & Eurofer & \multirow{15}{*}{ Helium } & \multirow{15}{*}{ TRRH 3/5 } & 268 & 247 & 280 \\
\hline & Outer SSJ tensile & Eurofer & & & 254 & 242 & 263 \\
\hline & Thermometry & $\mathrm{SiC}$ & & & 272 & 235 & 298 \\
\hline \multirow{3}{*}{$\begin{array}{c}9.25 \\
\left(300^{\circ} \mathrm{C}\right)\end{array}$} & Inner SSJ tensile & Eurofer & & & 304 & 283 & 316 \\
\hline & Outer SSJ tensile & Eurofer & & & 290 & 279 & 298 \\
\hline & Thermometry & $\mathrm{SiC}$ & & & 309 & 274 & 335 \\
\hline \multirow{3}{*}{$\begin{array}{c}9.20 \\
\left(325^{\circ} \mathrm{C}\right)\end{array}$} & Inner SSJ tensile & Eurofer & & & 330 & 309 & 341 \\
\hline & Outer SSJ tensile & Eurofer & & & 316 & 305 & 324 \\
\hline & Thermometry & $\mathrm{SiC}$ & & & 334 & 300 & 359 \\
\hline \multirow{3}{*}{$\begin{array}{c}9.15 \\
\left(350^{\circ} \mathrm{C}\right)\end{array}$} & Inner SSJ tensile & Eurofer & & & 360 & 339 & 371 \\
\hline & Outer SSJ tensile & Eurofer & & & 346 & 334 & 355 \\
\hline & Thermometry & $\mathrm{SiC}$ & & & 364 & 329 & 389 \\
\hline \multirow{3}{*}{$\begin{array}{c}9.00 \\
\left(375^{\circ} \mathrm{C}\right)\end{array}$} & Inner SSJ tensile & Eurofer & & & 388 & 368 & 399 \\
\hline & Outer SSJ tensile & Eurofer & & & 374 & 363 & 382 \\
\hline & Thermometry & $\mathrm{SiC}$ & & & 392 & 356 & 417 \\
\hline
\end{tabular}

Table 7. Predicted temperatures for the bend bar specimens and passive thermometry for each SSJ*/MPC specimen capsule case.

\begin{tabular}{|c|c|c|c|c|c|c|c|}
\hline \multirow{2}{*}{$\begin{array}{l}\text { Design diameter } \\
(\mathbf{m m})\end{array}$} & \multirow[b]{2}{*}{ Part name } & \multirow[b]{2}{*}{ Material } & \multirow[b]{2}{*}{ Fill gas } & \multirow{2}{*}{$\begin{array}{l}\text { Irradiation } \\
\text { location }\end{array}$} & \multicolumn{3}{|c|}{ Temperature $\left({ }^{\circ} \mathbf{C}\right)$} \\
\hline & & & & & Average & Minimum & Maximum \\
\hline \multirow{2}{*}{$\begin{array}{c}9.44 \\
\left(200^{\circ} \mathrm{C}\right)\end{array}$} & M4CVN & Eurofer & \multirow{14}{*}{ Helium } & \multirow{14}{*}{ TRRH 3/5 } & 196 & 169 & 210 \\
\hline & Thermometry & $\mathrm{SiC}$ & & & 192 & 185 & 195 \\
\hline \multirow{2}{*}{$\begin{array}{c}9.40 \\
\left(240^{\circ} \mathrm{C}\right) \\
\end{array}$} & M4CVN & Eurofer & & & 236 & 207 & 251 \\
\hline & Thermometry & $\mathrm{SiC}$ & & & 232 & 223 & 236 \\
\hline \multirow{2}{*}{$\begin{array}{c}9.35 \\
\left(275^{\circ} \mathrm{C}\right)\end{array}$} & M4CVN & Eurofer & & & 268 & 237 & 282 \\
\hline & Thermometry & $\mathrm{SiC}$ & & & 263 & 253 & 268 \\
\hline \multirow{2}{*}{$\begin{array}{c}9.30 \\
\left(300^{\circ} \mathrm{C}\right)\end{array}$} & M4CVN & Eurofer & & & 296 & 265 & 311 \\
\hline & Thermometry & $\mathrm{SiC}$ & & & 291 & 280 & 297 \\
\hline \multirow{2}{*}{$\begin{array}{c}9.27 \\
\left(330^{\circ} \mathrm{C}\right) \\
\end{array}$} & M4CVN & Eurofer & & & 321 & 289 & 337 \\
\hline & Thermometry & $\mathrm{SiC}$ & & & 316 & 304 & 322 \\
\hline \multirow{2}{*}{$\begin{array}{c}9.23 \\
\left(350^{\circ} \mathrm{C}\right)\end{array}$} & M4CVN & Eurofer & & & 347 & 313 & 363 \\
\hline & Thermometry & $\mathrm{SiC}$ & & & 341 & 328 & 348 \\
\hline \multirow{2}{*}{$\begin{array}{c}9.19 \\
\left(375^{\circ} \mathrm{C}\right)\end{array}$} & M4CVN & Eurofer & & & 371 & 337 & 387 \\
\hline & Thermometry & $\mathrm{SiC}$ & & & 366 & 352 & 373 \\
\hline
\end{tabular}

\section{AS-BUILT CAPSULE INFORMATION}

To June 2018, 21 irradiation capsules have been assembled and inserted into HFIR for irradiation. Out of the total number of capsules, seven (ES21, ES22, ES31-ES35) pertain to the screening phase, and the remaining fourteen capsules (ES01-ES07, ES21-ES27) pertain to the high dose phase. As part of the fabrication process, detailed dimensional, mass, and configuration data were produced to ensure that each capsule met the intended design requirements, the primary metrics being the customer-supplied loading lists and capsule design gas gaps. This report includes essential documentation providing these details 
(see attachments). More in-depth data such as fabrication and material inspection reports can be found in the fabrication files produce for the individual capsule assembly campaigns. These records are maintained by the Nuclear Engineering and Irradiation Testing group and are available upon request.

\section{SUMMARY AND CONCLUSIONS}

This work summarizes the capsule design, thermal analyses, and as-built capsule data for the EUROfusion Eurofer irradiation program. This document describes two specific designs: a modular, subsized tensile specimen format and a subsized Charpy test specimen format. These two capsule designs were optimized to produce holder-housing gas gaps that allow a specimen to reach temperatures between $200-400^{\circ} \mathrm{C}$. Each capsule configuration contains passive SiC thermometry that will provide temperature performance data during post-irradiation dilatometry to verify individual capsule temperatures. The irradiation program was divided into two phases: (1) low dose screening, and (2) high dose qualification. The former phase targeted $\sim 300^{\circ} \mathrm{C}$ as the average specimen temperatures at $2.5 \mathrm{dpa}$, and the latter phase targeted the full temperature range established for the program, with a cumulative irradiation dose of 25 dpa. As of the third quarter of the 2018 fiscal year, all requested capsules have been assembled, qualified, and inserted into HFIR for irradiation. Furthermore, at this writing, the screening phase capsule irradiation schedule and preparation of post-irradiation examination are underway.

\section{WORKS CITED}

[1] H. Tanigawa, E. Gaganidze, T. Hirose, M. Ando, S. Zinkle, R. Lindau and E. Diegele, "Development of benchmark reduced activation ferritic/martensitic steels for fusion energy applications," Nuclear Fusion, vol. 57, no. 9, 2017.

[2] A. Tavassoli, E. Diegele, R. Lindau, N. Luzginova and H. Tanigawa, "Current status and recent research achievements in ferritic/martensitic steels," Journal of Nuclear Materials, vol. 455, no. 13, pp. 269-276, 2014.

[3] A. Tavassoli, "Eurofer Steel, Development to Full Code Qualification," Procedia Engineering, vol. 55, pp. 300-308, 2013.

[4] Oak Ridge National Laboratory, "High Flux Isotope Reactor Technical Parameters," [Online]. Available: http://neutrons.ornl.gov/hfir/parameters. [Accessed 27 July 2016].

[5] A. Campbell, W. Porter, Y. Katoh and L. Snead, "Method for analyzing passive silicon carbide thermometry with a continuous dilatometer to determine irradiation temperature," Nuclear Instruments and Methods in Physics Research B, vol. 370, pp. 49-58, 2016.

[6] Oak RIdge National Laboratory, "In-Vessel Irradiation Experiment Facilities at HFIR," [Online]. Available: https://neutrons.ornl.gov/hfir/in-vessel-irradiation\#tr. [Accessed 2 July 2018].

[7] Thermal Hydraulics and Irradiation Engineering Group, "Target Capsule Housing Assembly," Oak Ridge National Laboratory, X3E020977A633, Rev. 0, Oak Ridge, TN, 2013.

[8] Thermal Hydraulics and Irradiation Engineering Group, "Target Capsule Housing/End Cap Detail," Oak Ridge National Laboratory, X3E020977A634, Rev. A, Oak Ridge, TN, 2013.

[9] Thermal Hydraulics and Irradiation Engineering Group, "Chevron-SSJ-MPCCVN-MPCThermometry," Oak Ridge National Laboratory, S16-18-FUSSAM01, Rev.1, Oak Ridge, TN, 2017.

[10] Thermal Hydraulics and Irradiation Engineering Group, "Rabbit Capsule Assembly," Oak Ridge National Laboratory, S16-20-ESTEEL, Rev. 1, Oak Ridge, TN, 2017.

[11] Thermal Hydraulics and Irradiation Engineering Group, "Rabbit Capsule Holder Assembly," Oak Ridge National Laboratory, S16-21-ESTEEL, Rev. 2, Oak Ridge, TN, 2017. 
[12] Thermal Hydraulics and Irradiation Engineering Group, "Rabbit Capsule Part Details," Oak Ridge National Laboratory, S16-22-ESTEEL, Rev. 1, Oak Ridge, TN, 2017.

[13] Thermal Hydraulics and Irradiation Engineering Group, "Rabbit Capsule Assembly," Oak Ridge National Laboratory, S16-23-ESTEELB, Rev. 1, Oak Ridge, TN., 2017.

[14] Thermal Hydraulics and Irradiation Engineering Group, "Rabbit Capsule Part Details," Oak Ridge National Laboratory, S16-24-ESTEELB, Rev. 1, Oak Ridge, TN, 2017.

[15] J. L. McDuffee, "Solve Macros for ANSYS Finite Element Models with Contact Elements," Oak Ridge National Laboratory, Thermal Hydraulics and Irradiation Engineering Group, DAC-11-13ANSYS02, Rev. 6, Oak Ridge, TN, 2016.

[16] CINDAS, LLC, "CINDAS, LLC: Global Benchmark for Critically Evaluated Materials Properties Data," [Online]. Available: http://cindasdata.com. [Accessed June 2017].

[17] MatWeb, LLC, "MatWeb: Material Property Data," [Online]. Available: http://matweb.com/. [Accessed June 2018].

[18] J. McDuffee, "Thermophysical Properties for AL6061," Oak Ridge National Laboratory, Thermal Hydraulics and Irradiation Engineering Group, DAC-10-03-PROP_AL6061, Rev.2, Oak Ridge, TN, 2013.

[19] R. Howard, "Thermophysical Properties for F82H Steel," Oak Ridge National Laboratory, Thermal Hydraulics and Irradiation Engineering Group, DAC-10-10-PROP_F82H, Rev. 1, Oak Ridge, TN, 2016.

[20] J. McDuffee, "Thermophysical Properties for 304 Stainless Steel," Oak Ridge National Laboratory, DAC-10-16-PROP_SS304, Rev.1, Oak Ridge, TN, 2013.

[21] J. McDuffee, "Thermophysical Properties for Flexible Graphite," Oak Ridge National Laboratory, Thermal Hydraulics and Irradiation Engineering Group, DAC-11-16-PROP_GRAFOIL, Rev. 0, Oak Ridge, TN, 2013.

[22] J. McDuffee, "Thermophysical Properties for Irradiated SiC," Oak Ridge National Laboratory, Thermal Hydraulics and Irradiation Engineering Group, DAC-10-06-PROP_SIC(IRR), Rev. 2, Oak Ridge, TN, 2013.

[23] J. McDuffee, "Thermophysical Properties for Molybdenum," Oak Ridge National Laboratory, Thermal Hydraulics and Irradiation Engineering Group, DAC-10-11-PROP_MOLY, Rev. 1, Oak Ridge, TN, 2013.

[24] J. McDuffee, "Thermophysical Properties for Helium," Oak Ridge National Laboratory, Thermal Hydraulics and Irradiation Engineering Group, DAC-10-02-PROP_HELIUM, Rev. 0, Oak Ridge, TN, 2010.

[25] K. Mergia and N. Boukos, "Structural, thermal, electrical and magnetic properties of Eurofer 97 steel," Journal of Nuclear Materials, vol. 373, pp. 1-8, 2008.

[26] R. Klueh, D. Gelles, A. K. S. Jitsukawa and G. Odette, "Ferritic/martensitic steels - overview of recent results," Journal of Nuclear Materials, Vols. 307-311, pp. 455-465, 2002.

[27] J. McDuffee, "Heat Transfer Coefficients and Bulk Temperatures for HFIR Rabbit Facilities," Oak Ridge National Laboratory, Thermal Hydraulics and Irradiation Engineering Group, DAC-11-01RAB03, Rev. 0, Oak Ridge, TN, 2011.

[28] J. McDuffee, "Heat Generation Rates for Various Rabbit Materials in the Flux Trap of HFIR," Oak Ridge National Laboratory, Thermal Hydraulics and Irradiation Engineering Group, C-HFIR-2012035, Rev. 0, Oak Ridge, TN, 2012. 
APPENDICES 


\section{APPENDIX A. ANSYS DESIGN REPORTS}


$9.40 \mathrm{~mm}$ holder diameter (tensile design)

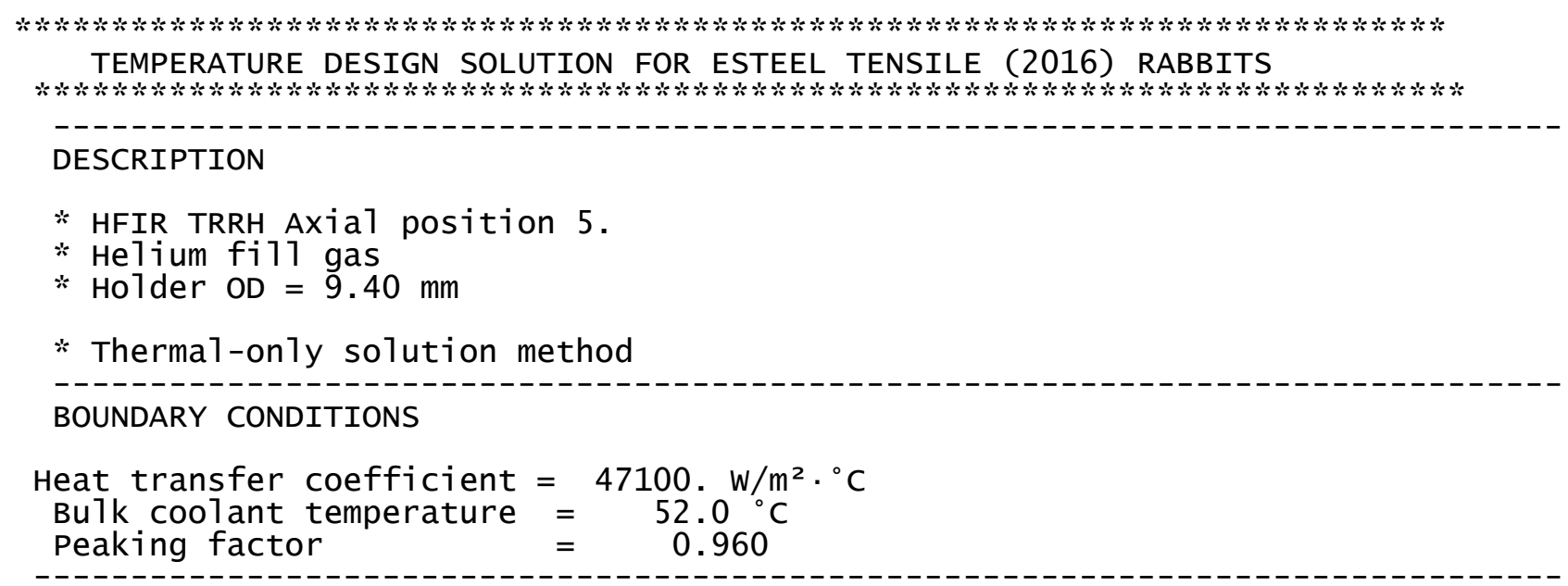

HEAT GENERATION

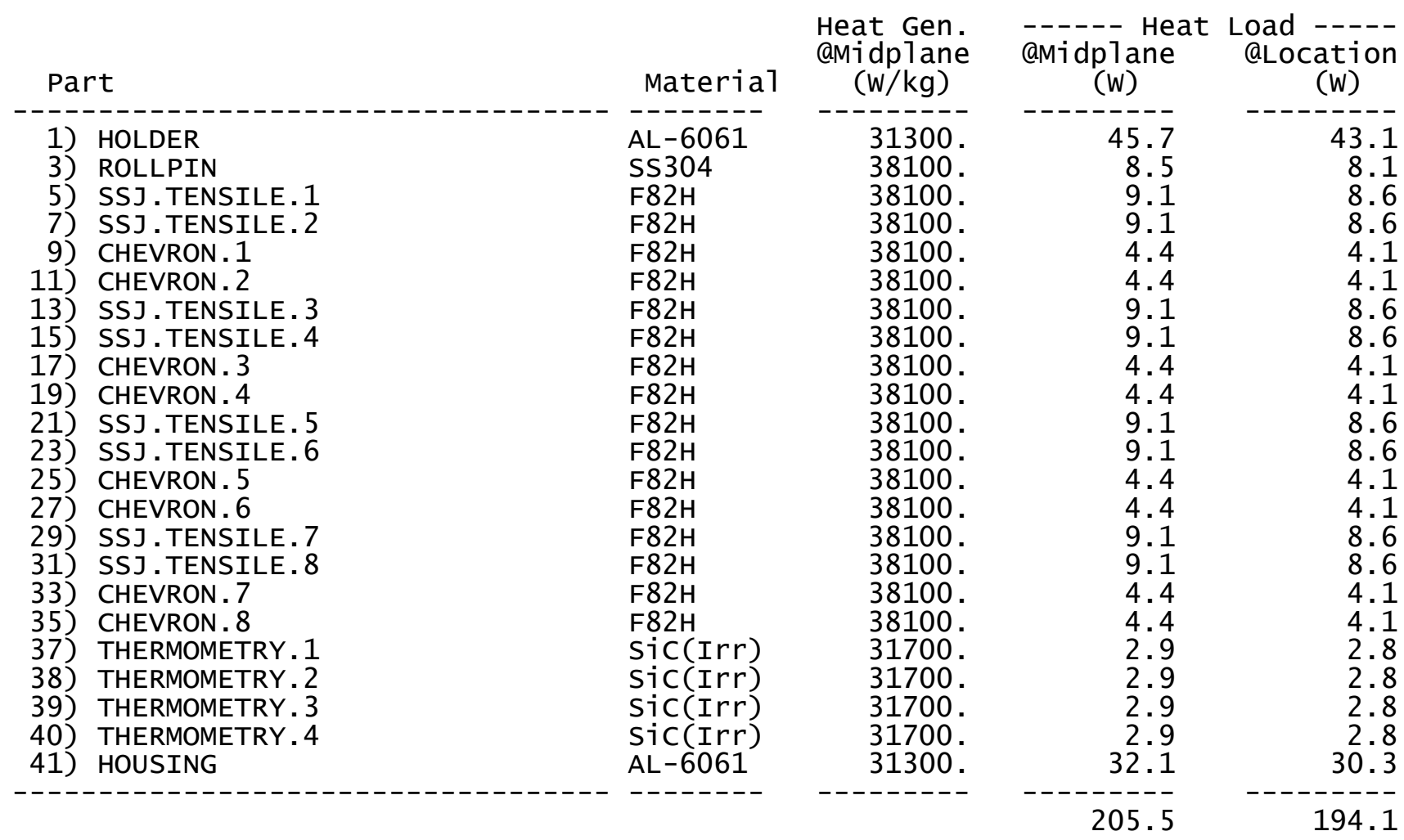

CAPSULE TEMPERATURE SUMMARY

\begin{tabular}{|c|c|c|c|c|c|c|c|}
\hline Name & & Material & Tavg & Tmin & Tmax & T. 025 & T. 975 \\
\hline $\begin{array}{r}1) \\
3) \\
5) \\
7) \\
9) \\
11) \\
13)\end{array}$ & $\begin{array}{l}\text { HOLDER } \\
\text { ROLLPIN } \\
\text { SSJ.TENSILE. } 1 \\
\text { SSJ.TENSILE. } 2 \\
\text { CHEVRON. } 1 \\
\text { CHEVRON. } 2 \\
\text { SSJ.TENSILE. } 3\end{array}$ & $\begin{array}{l}\text { AL-6061 } \\
\text { SS304 } \\
\text { F82H } \\
\text { F82H } \\
\text { F82H } \\
\text { F82H } \\
\text { F82H }\end{array}$ & $\begin{array}{l}158 . \\
254 \\
200 \\
186 \\
187 \\
201\end{array}$ & $\begin{array}{l}154 . \\
247 . \\
179 . \\
174 . \\
158 . \\
189 . \\
179 .\end{array}$ & $\begin{array}{l}166 . \\
264 . \\
212 . \\
195 . \\
195 . \\
208 . \\
212 .\end{array}$ & $\begin{array}{l}155 . \\
249 . \\
185 .\end{array}$ & $\begin{array}{l}161 . \\
262 . \\
210 . \\
192 . \\
193 . \\
206 . \\
210 .\end{array}$ \\
\hline
\end{tabular}


15) SSJ .TENSILE . 4

17) CHEVRON. 3

19) CHEVRON. 4

21) SSJ .TENSILE. 5

23) SSJ .TENSILE. 6

25) CHEVRON. 5

27) CHEVRON. 6

29) SSJ.TENSILE.7

31) SSJ .TENSILE. 8

33) CHEVRON.7

35) CHEVRON.8

37) THERMOMETRY.1

38) THERMOMETRY. 2

39) THERMOMETRY .3

40) THERMOMETRY.4

41) HOUSING
$\mathrm{F} 82 \mathrm{H}$

$\mathrm{F} 82 \mathrm{H}$

$\mathrm{F} 82 \mathrm{H}$

$\mathrm{F} 82 \mathrm{H}$

$\mathrm{F} 82 \mathrm{H}$

$\mathrm{F} 82 \mathrm{H}$

$\mathrm{F} 82 \mathrm{H}$

$\mathrm{F} 82 \mathrm{H}$

$\mathrm{F} 82 \mathrm{H}$

$\mathrm{F} 82 \mathrm{H}$

$\mathrm{F} 82 \mathrm{H}$

$\operatorname{SiC}(\operatorname{Ir} r)$

SiC(Irr)

SiC(Irr)

SiC(Irr)

AL-6061
186. 174. 194.

188. 180. 195. 184. 193.

200. 178. 212. 185. 210.

186. 175. 195. 178. 192 .

188. 181. 196. 184. 194.

200. 189. 207. 194. 205.

199. 178. 211. 184. 209.

185. 175. 193. 177. 191 .

182. 160. 190. 173. 188.

199. 187. 206. 193. 204.

203. 167. 230. 179. 220.

205. 169. 230. 180. 221.

205. 170. 234. 181. 222.

205. 169. 234. 181. 222.

PROPERTY SUMMARY AT THE AVERAGE PART TEMPERATURE

Name

1) HOLDER

3) ROLLPIN

5) SSJ.TENSILE.1

7) SSJ.TENSILE.2

9) CHEVRON. 1

11) CHEVRON. 2

13) SSJ.TENSILE. 3

15) SSJ.TENSILE. 4

17) CHEVRON. 3

19) CHEVRON. 4

21) SSJ.TENSILE. 5

23) SSJ.TENSILE. 6

25) CHEVRON. 5

27) CHEVRON. 6

29) SSJ .TENSILE. 7

31) SSJ.TENSILE.8

33) CHEVRON. 7

35) CHEVRON. 8

37) THERMOMETRY.1

38) THERMOMETRY.2

39) THERMOMETRY. 3

40) THERMOMETRY.4

41) HOUSING

\begin{tabular}{l} 
Material \\
\hline AL-6061 \\
SS304 \\
F82H \\
F82H \\
F82H \\
F82H \\
F82H \\
F82H \\
F82H \\
F82H \\
F82H \\
F82H \\
F82H \\
F82H \\
F82H \\
F82H \\
F82H \\
F82H \\
SiC(Irr) \\
SiC(Irr) \\
SiC(Irr) \\
SiC(Irr) \\
AL-6061
\end{tabular}

AL-6061
Thermat

$\left(\mathrm{W} / \mathrm{m} \cdot{ }^{\circ} \mathrm{C}\right)$ 176.000

18.772

33.231

33.148

33.155

33.232

33.231

33.149

33.162

33.231

33.230

33.148

33.163

33.230

33.223

33.142

33.126

33.224

6.101

6.101

6.100

6.101

166.661
Thermal

Exp. Coeff: Emis

$\left(\mu \mathrm{m} / \mathrm{m} \cdot{ }^{\circ} \mathrm{C}\right) \quad(---)$

$24.40 \quad 0.050$

$0.00 \quad 0.143$

$11.02 \quad 0.143$

$10.99 \quad 0.143$

$10.99 \quad 0.143$

$11.02 \quad 0.143$

$11.02 \quad 0.143$

$10.99 \quad 0.143$

$10.99 \quad 0.143$

$11.02 \quad 0.143$

$11.02 \quad 0.143$

$10.99 \quad 0.143$

$11.00 \quad 0.143$

$11.02 \quad 0.143$

$11.02 \quad 0.143$

$10.99 \quad 0.143$

$10.98 \quad 0.143$

$11.02 \quad 0.143$

$2.95 \quad 0.900$

$2.95 \quad 0.900$

$2.95 \quad 0.900$

$2.95 \quad 0.900$

STORED ENERGY SUMMARY AT THE AVERAGE PART TEMPERATURE

Name

1) HOLDER

3) ROLLPIN

5) SSJ.TENSILE.1

7) SSJ.TENSILE. 2

9) CHEVRON.1

11) CHEVRON. 2

13) SSJ.TENSILE. 3

15) SSJ.TENSILE. 4

\begin{tabular}{|c|c|c|c|c|}
\hline aterial & $\begin{array}{r}\text { Mass } \\
(\mathrm{g})\end{array}$ & $\begin{array}{l}\text { Tavg } \\
\left({ }^{\circ} \mathrm{C}\right)\end{array}$ & $\begin{array}{l}\text { Specific } \\
\text { Heat } \\
\left(\mathrm{J} / \mathrm{kg}^{\circ} \mathrm{C}\right)\end{array}$ & $\begin{array}{c}\text { Stored } \\
\text { Energy } \\
(\mathrm{J})\end{array}$ \\
\hline $\begin{array}{l}\text { SS3O4 } \\
\text { F82H } \\
\text { F82H } \\
\text { F82H } \\
\text { F82H } \\
\text { F82H } \\
\text { F82H }\end{array}$ & $\begin{array}{l}1.4 \\
0.2 \\
0.2 \\
0.2 \\
0.1 \\
0.1 \\
0.2 \\
0.2\end{array}$ & $\begin{array}{l}15 \\
25 \\
20 \\
18 \\
18 \\
20 \\
20 \\
18\end{array}$ & $\begin{array}{l}955 . \\
384 . \\
515 . \\
510 . \\
510 . \\
515 .\end{array}$ & $\begin{array}{r}193 . \\
20 . \\
22 . \\
20 .\end{array}$ \\
\hline
\end{tabular}


17) CHEVRON. 3

19) CHEVRON. 4

21) SSJ.TENSILE.5

23) SSJ .TENSILE.6

25) CHEVRON. 5

27) CHEVRON. 6

29) SSJ.TENSILE. 7

31) SSJ .TENSILE.8

33) CHEVRON. 7

35) CHEVRON .8

37) THERMOMETRY.1

38) THERMOMETRY.2

39) THERMOMETRY. 3

40) THERMOMETRY. 4

41) HOUSING

$\begin{array}{lrrrr}\text { F82H } & 0.115 & 188 . & 511 . & 10 . \\ \text { F82H } & 0.115 & 200 . & 515 . & 11 . \\ \text { F82H } & 0.238 & 200 . & 515 . & 22 . \\ \text { F82H } & 0.238 & 186 . & 510 . & 20 . \\ \text { F82H } & 0.115 & 188 . & 511 . & 10 . \\ \text { F82H } & 0.115 & 200 . & 515 . & 11 . \\ \text { F82H } & 0.238 & 199 . & 515 . & 22 . \\ \text { F82H } & 0.238 & 185 . & 509 . & 20 . \\ \text { F82H } & 0.115 & 182 . & 508 . & 9 . \\ \text { F82H } & 0.115 & 199 . & 515 . & 11 . \\ \text { SiC(Irr) } & 0.092 & 203 . & 946 . & 16 . \\ \text { SiC(Irr) } & 0.092 & 205 . & 947 . & 16 . \\ \text { SiC(Irr) } & 0.092 & 205 . & 948 . & 16 . \\ \text { SiC(Irr) } & 0.092 & 205 . & 948 . & 16 . \\ \text { AL-6061 } & 1.026 & 60 . & 885 . & 36 . \\ & ---1.05 & & & --1 \\ & 5.898 & & & 564 .\end{array}$

RADIAL DIMENSIONS AND GAP SUMMARY FOR THE CORE-HOUSING GAP

CONTACT SUMMARY FOR CONTACT ID 83: Frictionless - HOLDER TO HOUSING

Contact surface material:

Target surface material:

Interstitial gas:

Effective surface roughness:

Effective asperity slope:

Effective microhardness:

$$
\begin{aligned}
& \text { AL-6061 } \\
& \text { AL-6061 } \\
& \text { He1 1 um } \\
& 2.263 \mu \mathrm{m} \\
& 0.214 \mathrm{rad} \\
& 1.220 \mathrm{GPa}
\end{aligned}
$$

\begin{tabular}{|c|c|c|c|}
\hline & Average & Minimum & Maximum \\
\hline $\begin{array}{l}\text { direct results } \\
\text { Contact status } \\
\text { Contact temperature }\left({ }^{\circ} \mathrm{C}\right) \\
\text { Target temperature }\left({ }^{\circ} \mathrm{C}\right) \\
\text { Geometric gas gap }(\mu \mathrm{m}) \\
\text { Contact pressure }(\mathrm{MPa}) \\
\text { Gap conduction heat flux }\left(\mathrm{kW} / \mathrm{m}^{2}\right) \\
\text { Radiation heat flux }\left(\mathrm{kW} / \mathrm{m}^{2}\right) \\
\text { Contact conduction heat flux }\left(\mathrm{kW} / \mathrm{m}^{2}\right) \\
\text { Total heat flux }\left(\mathrm{kW} / \mathrm{m}^{2}\right) \\
\text { Thermal contact conductance }\left(\mathrm{W} / \mathrm{m}^{2} \cdot \mathrm{C}\right)\end{array}$ & $\begin{array}{r}1.000 \\
156.965 \\
60.729 \\
59.965 \\
0.000 \\
339.548 \\
0.000 \\
0.000 \\
339.548 \\
3528.169\end{array}$ & $\begin{array}{r}1.000 \\
154.363 \\
60.058 \\
59.689 \\
0.000 \\
327.342 \\
0.000 \\
0.000 \\
327.342 \\
3487.045\end{array}$ & $\begin{array}{r}1.000 \\
158.462 \\
60.906 \\
60.323 \\
0.000 \\
346.952 \\
0.000 \\
0.000 \\
346.952 \\
3557.529\end{array}$ \\
\hline $\begin{array}{l}\text { Effective gas gap }(\mu \mathrm{m}) \\
\text { Contact therma1 jump distance }(\mu \mathrm{m}) \\
\text { Target therma1 jump distance }(\mu \mathrm{m}) \\
\text { Effective contact pressure }(\mathrm{MPa}) \\
\text { Pressure index } \\
\text { Gas thermal conductivity }\left(\mathrm{W} / \mathrm{m} \cdot{ }^{\circ} \mathrm{C}\right) \\
\text { Solid spot conductance }\left(\mathrm{W} / \mathrm{m}^{2} \cdot \mathrm{C}\right) \\
\text { Gas gap conductance }\left(\mathrm{W} / \mathrm{m}^{2} \cdot \mathrm{C}\right)\end{array}$ & $\begin{array}{r}48.896 \\
1.079 \\
1.020 \\
0.000 \\
13.534 \\
0.180 \\
0.000 \\
3528.660\end{array}$ & $\begin{array}{r}48.498 \\
1.073 \\
1.016 \\
0.000 \\
13.534 \\
0.180 \\
0.000 \\
3488.720\end{array}$ & $\begin{array}{r}49.375 \\
1.082 \\
1.023 \\
0.000 \\
13.534 \\
0.180 \\
0.000 \\
3557.215\end{array}$ \\
\hline
\end{tabular}

Contact status codes:

$0=$ open/no heat transfer, $1=$ near-field contact $2=$ closed and sliding, $\quad 3=c l o s e d$ and sticking 
$9.38 \mathrm{~mm}$ holder diameter (tensile design)

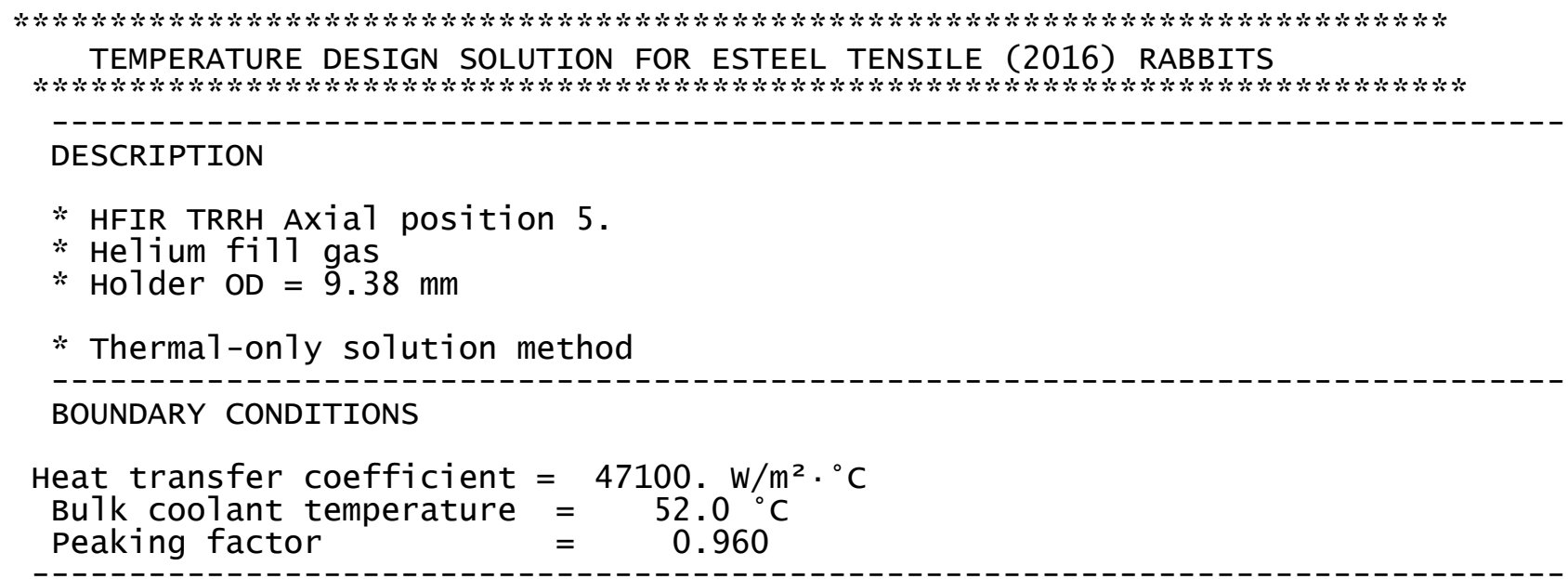

HEAT GENERATION

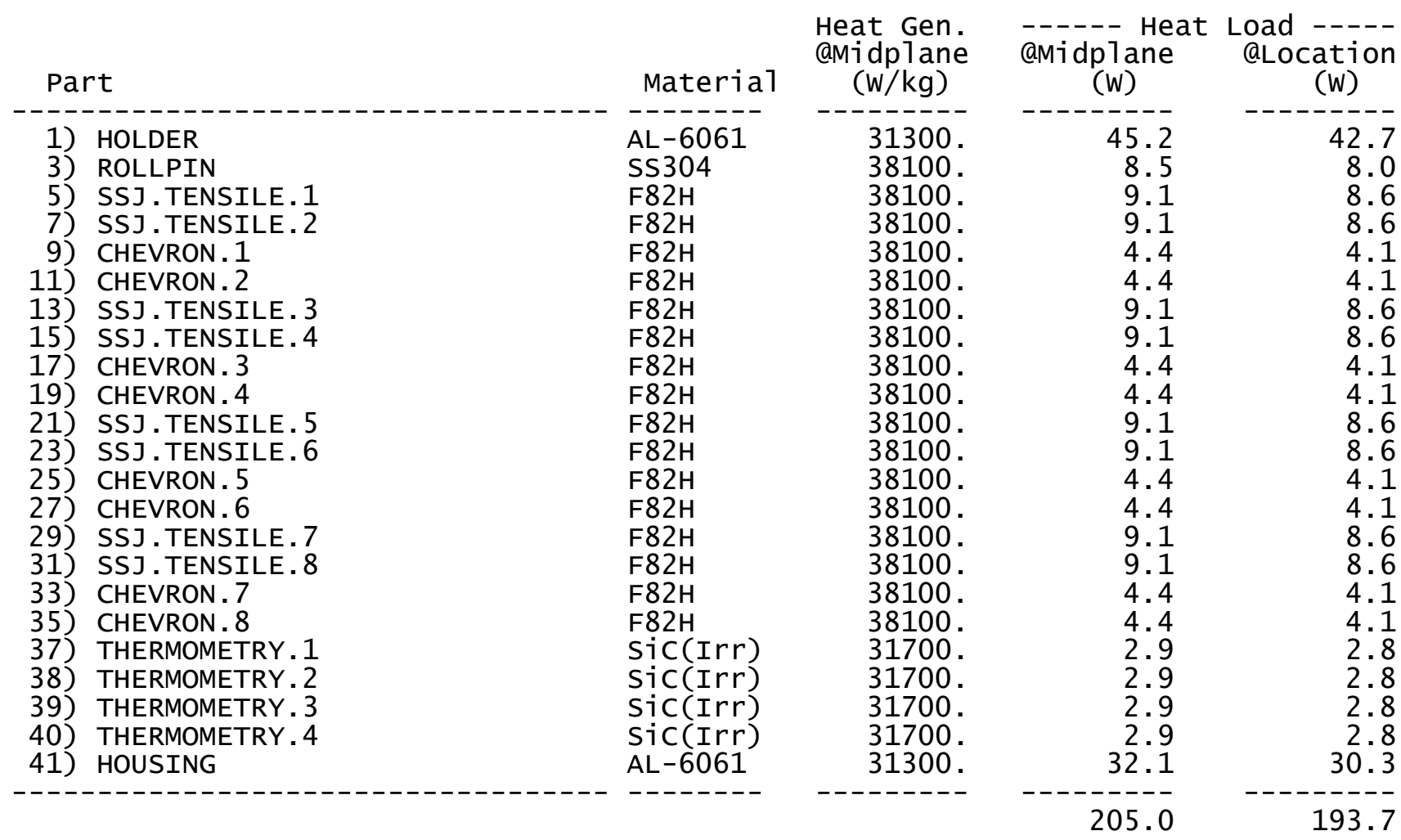

CAPSULE TEMPERATURE SUMMARY

\begin{tabular}{|c|c|c|c|c|c|c|c|}
\hline Name & & Material & Tavg & Tmin & Tmax & T. 025 & Т. 975 \\
\hline $\begin{array}{r}1) \\
3) \\
5) \\
7) \\
9) \\
11) \\
13)\end{array}$ & $\begin{array}{l}\text { HOLDER } \\
\text { ROLLPIN } \\
\text { SSJ.TENSILE. } 1 \\
\text { SSJ.TENSILE. } 2 \\
\text { CHEVRON. } 1 \\
\text { CHEVRON. } 2 \\
\text { SSJ.TENSILE. } 3\end{array}$ & $\begin{array}{l}\text { AL-6061 } \\
\text { SS304 } \\
\text { F82H } \\
\text { F82H } \\
\text { F82H } \\
\text { F82H } \\
\text { F82H }\end{array}$ & $\begin{array}{l}173 . \\
267 . \\
214 . \\
200 . \\
200 . \\
214 . \\
214 .\end{array}$ & $\begin{array}{l}169 . \\
261 . \\
193 \\
188 . \\
171 . \\
202 . \\
193\end{array}$ & $\begin{array}{l}182 . \\
278 \\
226 \\
208 \\
208 \\
221 \\
226\end{array}$ & $\begin{array}{l}170 . \\
263 . \\
198 . \\
192 . \\
193 . \\
208 . \\
199 .\end{array}$ & $\begin{array}{l}175 . \\
275 . \\
224 . \\
206 . \\
206 . \\
219 . \\
224 .\end{array}$ \\
\hline
\end{tabular}


15) SSJ .TENSILE . 4

17) CHEVRON. 3

19) CHEVRON. 4

21) SSJ .TENSILE. 5

23) SSJ .TENSILE. 6

25) CHEVRON. 5

27) CHEVRON. 6

29) SSJ.TENSILE.7

31) SSJ .TENSILE. 8

33) CHEVRON.7

35) CHEVRON.8

37) THERMOMETRY.1

38) THERMOMETRY. 2

39) THERMOMETRY .3

40) THERMOMETRY.4

41) HOUSING
F82H 200. 190. 209. 192. 206.

$\mathrm{F} 82 \mathrm{H} 202.2194 .209 .2198 .208$.

$\mathrm{F} 82 \mathrm{H} 215.203 .222 .208 .220$.

$\mathrm{F} 82 \mathrm{H} 214.2192 .226 .2199 .22$.

$\mathrm{F} 82 \mathrm{H} 200.2189 .209 .2192 .206$.

$\mathrm{F} 82 \mathrm{H} 203.2195 .210 .2198 .208$.

F82H 214. 203. 221. 208. 219.

$\mathrm{F} 82 \mathrm{H} 214.2193 .225 .2198 .224$.

$\mathrm{F} 82 \mathrm{H} 200.2189 .208 .2191 .206$.

F82H 202. 193. 209. 197. 207.

F82H 214. 199. 221. 208. 219.

SiC(Irr) 219. 182. 244. 194. 235.

SiC(Irr) 219. 184. 244. 194. 235.

Sic(Irr) 219. 184. 248. 195. 236.

SiC(Irr) 219. 183. 248. 195. 236.

PROPERTY SUMMARY AT THE AVERAGE PART TEMPERATURE

Name

1) HOLDER

3) ROLLPIN

5) SSJ.TENSILE.1

7) SSJ.TENSILE.2

9) CHEVRON.1

11) CHEVRON. 2

13) SSJ.TENSILE. 3

15) SSJ.TENSILE. 4

17) CHEVRON. 3

19) CHEVRON. 4

21) SSJ.TENSILE. 5

23) SSJ.TENSILE. 6

25) CHEVRON. 5

27) CHEVRON. 6

29) SSJ .TENSILE. 7

31) SSJ.TENSILE.8

33) CHEVRON.7

35) CHEVRON. 8

37) THERMOMETRY.1

38) THERMOMETRY.2

39) THERMOMETRY. 3

40) THERMOMETRY.4

41) HOUSING

\begin{tabular}{l} 
Material \\
\hline AL-6061 \\
SS304 \\
F82H \\
F82H \\
F82H \\
F82H \\
F82H \\
F82H \\
F82H \\
F82H \\
F82H \\
F82H \\
F82H \\
F82H \\
F82H \\
F82H \\
F82H \\
F82H \\
SiC(Irr) \\
SiC(Irr) \\
SiC(Irr) \\
SiC(Irr)
\end{tabular}

AL-6061
Therma1 cond.

$\left(\mathrm{W} / \mathrm{m} \cdot{ }^{\circ} \mathrm{C}\right)$ 176.000

18.963

33.304

33.226

33.230

33.304

33.306

33.229

33.241

33.308

33.305

33.228

33.243

33.305

33.303

33.226

33.238

33.304

6.095

6.095

6.095

6.095

166.659
Thermal

Exp. Coeff: Emis $\left(\mu \mathrm{m} / \mathrm{m} \cdot{ }^{\circ} \mathrm{C}\right)$

$24.49 \quad 0.051$

$0.00 \quad 0.143$

$11.04 \quad 0.143$

$11.02 \quad 0.143$

$11.02 \quad 0.143$

$11.04 \quad 0.143$

$11.04 \quad 0.143$

$11.02 \quad 0.143$

$11.02 \quad 0.143$

$11.05 \quad 0.143$

$11.04 \quad 0.143$

$11.02 \quad 0.143$

$11.02 \quad 0.143$

$11.04 \quad 0.143$

$11.04 \quad 0.143$

$11.02 \quad 0.143$

$11.02 \quad 0.143$

$11.04 \quad 0.143$

$3.00 \quad 0.900$

$3.00 \quad 0.900$

$3.00 \quad 0.900$

$3.00 \quad 0.900$

STORED ENERGY SUMMARY AT THE AVERAGE PART TEMPERATURE

Name

1) HOLDER

3) ROLLPIN

5) SSJ.TENSILE.1

7) SSJ.TENSILE. 2

9) CHEVRON.1

11) CHEVRON. 2

13) SSJ.TENSILE. 3

15) SSJ.TENSILE. 4

\begin{tabular}{|c|c|c|c|c|}
\hline aterial & $\begin{array}{r}\text { Mass } \\
(\mathrm{g})\end{array}$ & $\begin{array}{l}\text { Tavg } \\
\left({ }^{\circ} \mathrm{C}\right)\end{array}$ & $\begin{array}{l}\text { Specific } \\
\text { Heat } \\
\left(\mathrm{J} / \mathrm{kg}^{\circ} \mathrm{C}\right)\end{array}$ & $\begin{array}{c}\text { Stored } \\
\text { Energy } \\
(\mathrm{J})\end{array}$ \\
\hline $\begin{array}{l}\text { SS3O4 } \\
\text { F82H } \\
\text { F82H } \\
\text { F82H } \\
\text { F82H } \\
\text { F82H } \\
\text { F82H }\end{array}$ & $\begin{array}{l}1.445 \\
0.223 \\
0.238 \\
0.238 \\
0.115 \\
0.115 \\
0.238 \\
0.238\end{array}$ & $\begin{array}{l}173 . \\
267 . \\
214 . \\
200 . \\
200 . \\
214 . \\
214 . \\
200 .\end{array}$ & $\begin{array}{l}965 . \\
385 . \\
520 . \\
515 . \\
515 . \\
520 . \\
520 .\end{array}$ & $\begin{array}{r}213 . \\
21 . \\
24 . \\
22 . \\
11 .\end{array}$ \\
\hline
\end{tabular}


17) CHEVRON. 3

19) CHEVRON. 4

21) SSJ.TENSILE.5

23) SSJ .TENSILE.6

25) CHEVRON. 5

27) CHEVRON. 6

29) SSJ.TENSILE. 7

31) SSJ .TENSILE.8

33) CHEVRON. 7

35) CHEVRON .8

37) THERMOMETRY.1

38) THERMOMETRY.2

39) THERMOMETRY. 3

40) THERMOMETRY. 4

41) HOUSING

$\begin{array}{lrrrr}\text { F82H } & 0.115 & 202 . & 516 . & 11 . \\ \text { F82H } & 0.115 & 215 . & 520 . & 12 . \\ \text { F82H } & 0.238 & 214 . & 520 . & 24 . \\ \text { F82H } & 0.238 & 200 . & 515 . & 22 . \\ \text { F82H } & 0.115 & 203 . & 516 . & 11 . \\ \text { F82H } & 0.115 & 214 . & 520 . & 12 . \\ \text { F82H } & 0.238 & 214 . & 520 . & 24 . \\ \text { F82H } & 0.238 & 200 . & 515 . & 22 . \\ \text { F82H } & 0.115 & 202 . & 516 . & 11 . \\ \text { F82H } & 0.115 & 214 . & 520 . & 12 . \\ \text { SiC(Irr) } & 0.092 & 219 . & 958 . & 18 . \\ \text { SiC(Irr) } & 0.092 & 219 . & 958 . & 18 . \\ \text { SiC(Irr) } & 0.092 & 219 . & 958 . & 18 . \\ \text { SiC(Irr) } & 0.092 & 219 . & 958 . & 18 . \\ \text { AL-6061 } & 1.026 & 60 . & 884 . & 36 . \\ & ---1.0- & & & --14 .\end{array}$

RADIAL DIMENSIONS AND GAP SUMMARY FOR THE CORE-HOUSING GAP

CONTACT SUMMARY FOR CONTACT ID 83: Frictionless - HOLDER TO HOUSING

Contact surface material:

Target surface material:

Interstitial gas:

Effective surface roughness:

Effective asperity slope:

Effective microhardness:

$$
\begin{aligned}
& \text { AL-6061 } \\
& \text { AL-6061 } \\
& \text { He1jum } \\
& 2.263 \mu \mathrm{m} \\
& 0.214 \mathrm{rad} \\
& 1.220 \mathrm{GPa}
\end{aligned}
$$

\begin{tabular}{|c|c|c|c|}
\hline & Average & Minimum & Maximum \\
\hline$\sim \sim \sim \sim \sim \sim \sim \sim \sim \sim m$ direct results $\sim$ & 1000 & 1000 & 1000 \\
\hline Contact temperature $\left({ }^{\circ} \mathrm{C}\right)$ & 171.241 & 168.606 & 172.669 \\
\hline Target temperature $\left({ }^{\circ} \mathrm{C}\right)$ & 60.711 & 60.079 & 60.892 \\
\hline Geometric gas gap $(\mu \mathrm{m})$ & 69.965 & 69.383 & 70.295 \\
\hline Contact pressure (MPa) & 0.000 & 0.000 & 0.000 \\
\hline Gap conduction heat flux $\left(\mathrm{kW} / \mathrm{m}^{2}\right)$ & 339.447 & 328.766 & 346.527 \\
\hline Radiation heat flux $\left(\mathrm{kW} / \mathrm{m}^{2}\right)$ & 0.000 & 0.000 & 0.000 \\
\hline Contact conduction heat flux $\left(\mathrm{kW} / \mathrm{m}^{2}\right)$ & 0.000 & 0.000 & 0.000 \\
\hline Total heat flux $\left(\mathrm{kW} / \mathrm{m}^{2}\right)$ & 339.447 & 328.76 & 346.527 \\
\hline Therma1 contact conductance $\left(\mathrm{W} / \mathrm{m}^{2} \cdot \mathrm{c}\right)$ & 3071.021 & 3039.34 & 3109.63 \\
\hline Effective gas gap $(\mu \mathrm{m})$ & 57.221 & 56.530 & 57.70 \\
\hline Contact thermal jump distance $(\mu \mathrm{m})$ & & & 11 \\
\hline Target thermal jump distance $(\mu \mathrm{m})$ & 1.044 & 1.039 & 1 \\
\hline Effective contact pressure (MPa) & & 0 & 0.000 \\
\hline Pressure index & 13.534 & 13.534 & 13.5 \\
\hline thermat conduc & & 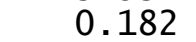 & \\
\hline Solid spot conducta & 0 & 0.00 & 0.00 \\
\hline Gas gap conductance & 3072.8 & 3041.380 & 111.320 \\
\hline
\end{tabular}

Contact status codes:

$0=$ open/no heat transfer, $1=$ near-field contact $2=$ closed and sliding, $\quad 3=$ closed and sticking 
$9.34 \mathrm{~mm}$ holder diameter (tensile design)

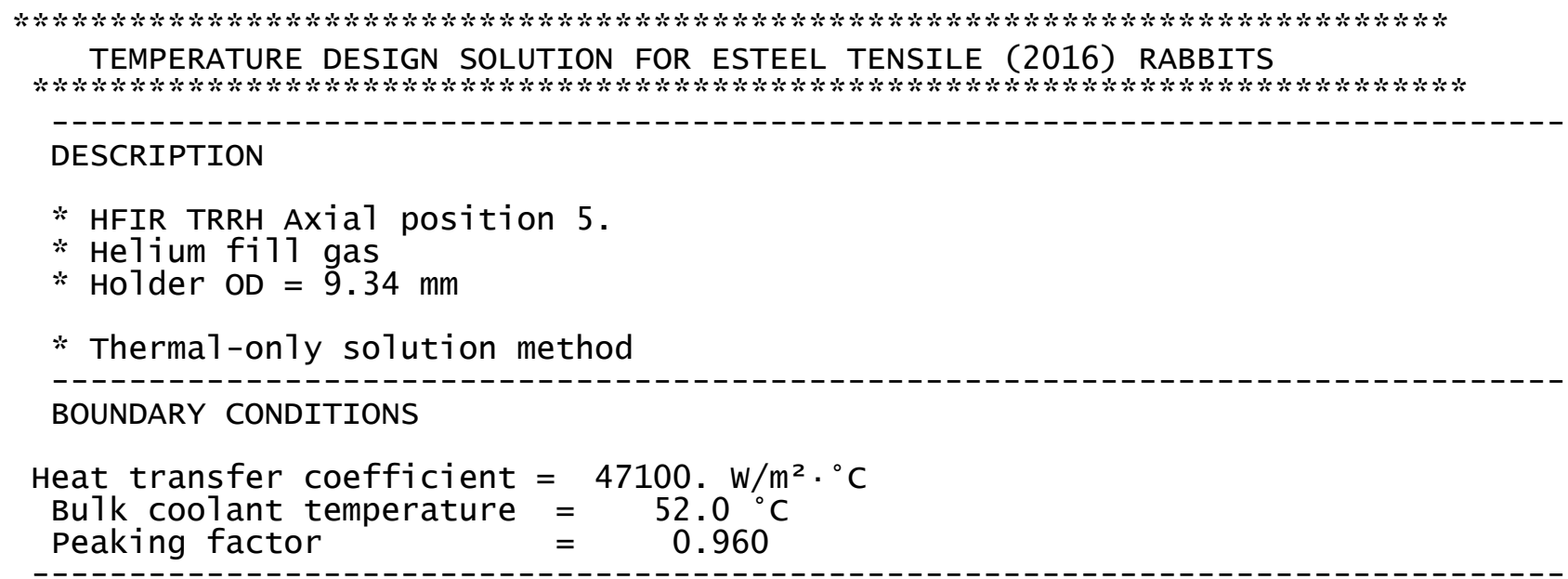

HEAT GENERATION

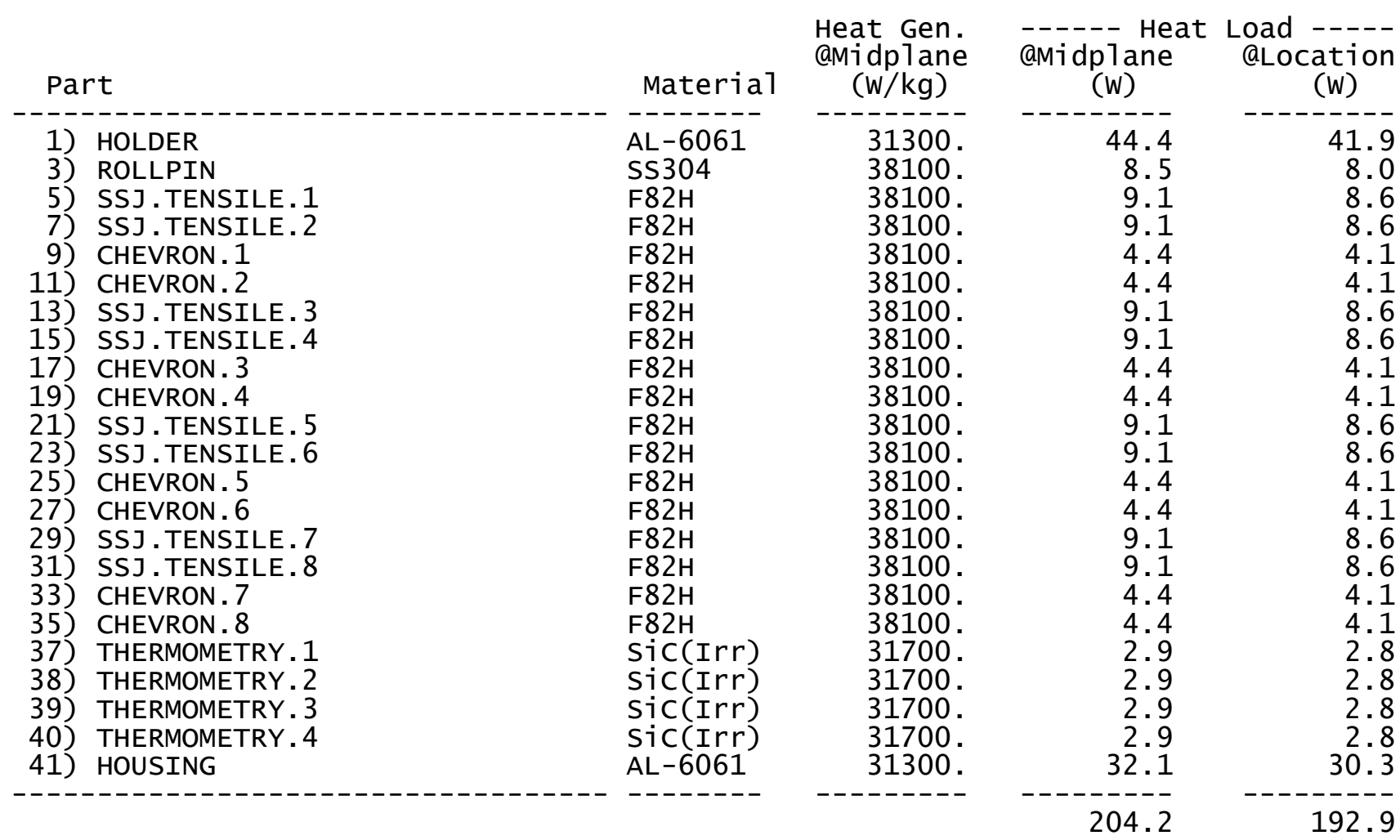

CAPSULE TEMPERATURE SUMMARY

\begin{tabular}{|c|c|c|c|c|c|c|c|}
\hline Name & & Material & Tavg & Tmin & Tmax & T. 025 & Т. 975 \\
\hline $\begin{array}{r}1) \\
3) \\
5) \\
7) \\
9) \\
11) \\
13)\end{array}$ & $\begin{array}{l}\text { HOLDER } \\
\text { ROLLPIN } \\
\text { SSJ.TENSILE. } 1 \\
\text { SSJ.TENSILE. } 2 \\
\text { CHEVRON. } 1 \\
\text { CHEVRON. } 2 \\
\text { SSJ.TENSILE. } 3\end{array}$ & $\begin{array}{l}\text { AL-6061 } \\
\text { SS304 } \\
\text { F82H } \\
\text { F82H } \\
\text { F82H } \\
\text { F82H } \\
\text { F82H }\end{array}$ & $\begin{array}{l}200 . \\
294 . \\
241 \\
227 \\
227 \\
241 \\
241\end{array}$ & $\begin{array}{l}196 . \\
288 . \\
220 . \\
215 . \\
199 . \\
229 . \\
220 .\end{array}$ & $\begin{array}{l}206 . \\
304 . \\
253 \\
236 \\
235 \\
248 \\
253\end{array}$ & $\begin{array}{l}197 . \\
289 . \\
226 . \\
219 . \\
220 .\end{array}$ & $\begin{array}{l}203 . \\
302 . \\
251 . \\
233 . \\
233 . \\
246 . \\
251 .\end{array}$ \\
\hline
\end{tabular}


15) SSJ .TENSILE . 4

17) CHEVRON. 3

19) CHEVRON. 4

21) SSJ .TENSILE. 5

23) SSJ .TENSILE. 6

25) CHEVRON. 5

27) CHEVRON. 6

29) SSJ.TENSILE.7

31) SSJ.TENSILE. 8

33) CHEVRON.7

35) CHEVRON.8

37) THERMOMETRY.1

38) THERMOMETRY. 2

39) THERMOMETRY .3

40) THERMOMETRY.4

41) HOUSING
$\mathrm{F} 82 \mathrm{H}$

$\mathrm{F} 82 \mathrm{H}$

$\mathrm{F} 82 \mathrm{H}$

$\mathrm{F} 82 \mathrm{H}$

$\mathrm{F} 82 \mathrm{H}$

$\mathrm{F} 82 \mathrm{H}$

$\mathrm{F} 82 \mathrm{H}$

$\mathrm{F} 82 \mathrm{H}$

$\mathrm{F} 82 \mathrm{H}$

$\mathrm{F} 82 \mathrm{H}$

$\mathrm{F} 82 \mathrm{H}$

$\operatorname{SiC}(\operatorname{Ir} r)$

SiC(Irr)

SiC(Irr)

SiC(Irr)
227. 215. 236. 219. 233.

229. 221. 236. 225. 235.

241. 229. 249. 235. 247.

241. 219. 252. 226. 251.

227. 217. 235. 219. 233.

230. 222. 237. 225. 235 .

242. 230. 249. 235. 247.

241. 221. 253. 226. 251 .

227. 216. 236. 219. 233.

230. 221. 237. 225. 235.

242. 230. 249. 235. 247 .

246. 211. 271. 222. 262.

246. 210. 271. 222. 262.

246. 209. 275. 222. 262 .

246. 210. 275. 222. 263.

60. 59. 61. 59. 61.

PROPERTY SUMMARY AT THE AVERAGE PART TEMPERATURE

Name

1) HOLDER

3) ROLLPIN

5) SSJ.TENSILE. 1

7) SSJ.TENSILE.2

9) CHEVRON.1

11) CHEVRON. 2

13) SSJ.TENSILE. 3

15) SSJ.TENSILE. 4

17) CHEVRON. 3

19) CHEVRON. 4

21) SSJ.TENSILE. 5

23) SSJ.TENSILE. 6

25) CHEVRON. 5

27) CHEVRON. 6

29) SSJ .TENSILE. 7

31) SSJ.TENSILE.8

33) CHEVRON.7

35) CHEVRON. 8

37) THERMOMETRY.1

38) THERMOMETRY.2

39) THERMOMETRY. 3

40) THERMOMETRY.4

41) HOUSING

\begin{tabular}{l} 
Material \\
\hline AL-6061 \\
SS304 \\
F82H \\
F82H \\
F82H \\
F82H \\
F82H \\
F82H \\
F82H \\
F82H \\
F82H \\
F82H \\
F82H \\
F82H \\
F82H \\
F82H \\
F82H \\
F82H \\
SiC(Irr) \\
SiC(Irr) \\
SiC(Irr) \\
SiC(Irr)
\end{tabular}

AL-6061

\section{Thermat \\ cond.}

$\left(\mathrm{W} / \mathrm{m} \cdot{ }^{\circ} \mathrm{C}\right)$ 176.000

19.332

33.448

33.372

33.374

33.447

33.448

33.373

33.385

33.449

33.449

33.373

33.387

33.450

33.449

33.372

33.387

33.450

6.085

6.085

6.085

6.085

166.655
Thermal

Exp. Coeff. Emis $\left(\mu \mathrm{m} / \mathrm{m} \cdot{ }^{\circ} \mathrm{C}\right)(---)$

$24.66 \quad 0.052$

$0.00 \quad 0.143$

$11.09 \quad 0.143$

$11.07 \quad 0.143$

$11.07 \quad 0.143$

$11.09 \quad 0.143$

$11.09 \quad 0.143$

$11.07 \quad 0.143$

$11.07 \quad 0.143$

$11.09 \quad 0.143$

$11.09 \quad 0.143$

$11.07 \quad 0.143$

$11.07 \quad 0.143$

$11.09 \quad 0.143$

$11.09 \quad 0.143$

$11.07 \quad 0.143$

$11.07 \quad 0.143$

$11.09 \quad 0.143$

$3.09 \quad 0.900$

$3.09 \quad 0.900$

$3.09 \quad 0.900$

$3.09 \quad 0.900$

STORED ENERGY SUMMARY AT THE AVERAGE PART TEMPERATURE

Name

1) HOLDER

3) ROLLPIN

5) SSJ.TENSILE.1

7) SSJ.TENSILE. 2

9) CHEVRON.1

11) CHEVRON. 2

13) SSJ.TENSILE. 3

15) SSJ.TENSILE. 4

$\begin{array}{lrrrr}\text { Material } & \begin{array}{c}\text { Mass } \\ (\mathrm{g})\end{array} & \begin{array}{c}\text { Tavg } \\ \left({ }^{\circ} \mathrm{C}\right)\end{array} & \begin{array}{c}\text { Specific } \\ \mathrm{Heat} \\ \left(\mathrm{J} / \mathrm{kg}^{\circ} \mathrm{C}\right)\end{array} & \begin{array}{c}\text { Stored } \\ \text { Energy } \\ (\mathrm{J})\end{array} \\ ------ & ---- & ---- & ----- & ----- \\ \text { AL-6061 } & 1.419 & 200 . & 983 . & 252 . \\ \text { SS304 } & 0.223 & 294 . & 387 . & 24 . \\ \text { F82H } & 0.238 & 241 . & 530 . & 28 . \\ \text { F82H } & 0.238 & 227 . & 525 . & 26 . \\ \text { F82H } & 0.115 & 227 . & 525 . & 12 . \\ \text { F82H } & 0.115 & 241 . & 530 . & 13 . \\ \text { F82H } & 0.238 & 241 . & 530 . & 28 . \\ \text { F82H } & 0.238 & 227 . & 525 . & 26 .\end{array}$


17) CHEVRON. 3

19) CHEVRON. 4

21) SSJ.TENSILE.5

23) SSJ .TENSILE.6

25) CHEVRON. 5

27) CHEVRON. 6

29) SSJ.TENSILE. 7

31) SSJ .TENSILE.8

33) CHEVRON. 7

35) CHEVRON .8

37) THERMOMETRY.1

38) THERMOMETRY.2

39) THERMOMETRY. 3

40) THERMOMETRY. 4

41) HOUSING

$\begin{array}{lrrrr}\text { F82H } & 0.115 & 229 . & 525 . & 13 . \\ \text { F82H } & 0.115 & 241 . & 530 . & 13 . \\ \text { F82H } & 0.238 & 241 . & 530 . & 28 . \\ \text { F82H } & 0.238 & 227 . & 525 . & 26 . \\ \text { F82H } & 0.115 & 230 . & 526 . & 13 . \\ \text { F82H } & 0.115 & 242 . & 530 . & 13 . \\ \text { F82H } & 0.238 & 241 . & 530 . & 28 . \\ \text { F82H } & 0.238 & 227 . & 525 . & 26 . \\ \text { F82H } & 0.115 & 230 . & 526 . & 13 . \\ \text { F82H } & 0.115 & 242 . & 530 . & 13 . \\ \text { SiC(Irr) } & 0.092 & 246 . & 978 . & 20 . \\ \text { SiC(Irr) } & 0.092 & 246 . & 978 . & 20 . \\ \text { SiC(Irr) } & 0.092 & 246 . & 978 . & 20 . \\ \text { SiC(Irr) } & 0.092 & 246 . & 978 . & 20 . \\ \text { AL-6061 } & 1.026 & 60 . & 884 . & 36 . \\ & ---1.0- & & & --- \\ & 5.858 & & & 712 .\end{array}$

RADIAL DIMENSIONS AND GAP SUMMARY FOR THE CORE-HOUSING GAP

CONTACT SUMMARY FOR CONTACT ID 83: Frictionless - HOLDER TO HOUSING

Contact surface material:

Target surface material:

Interstitial gas:

Effective surface roughness:

Effective asperity slope:

Effective microhardness:

\begin{tabular}{|c|c|c|c|}
\hline & Average & Minimum & Maximum \\
\hline \\
\hline $\begin{array}{l}\text { Contact status } \\
\text { Contact temperature }\left({ }^{\circ} \mathrm{C}\right)\end{array}$ & $\begin{array}{r}1.000 \\
198.948\end{array}$ & $\begin{array}{r}1.000 \\
196.174\end{array}$ & $\begin{array}{r}1.000 \\
200.405\end{array}$ \\
\hline Target temperature $\left({ }^{\circ} \mathrm{C}\right)$ & 60.681 & 60.077 & 60.826 \\
\hline Geometric gas gap $(\mu \mathrm{m})$ & 89.963 & 89.547 & 90.283 \\
\hline Contact pressure (MPa) & 0.000 & 0.000 & 0.000 \\
\hline Gap conduction heat flux $\left(\mathrm{kW} / \mathrm{m}^{2}\right)$ & 339.33 & 330.169 & 344.725 \\
\hline Radiation heat flux $\left(\mathrm{kW} / \mathrm{m}^{2}\right)$ & 0.0 & 0.000 & \\
\hline Contact conduction heat flux $\left(\mathrm{kW} / \mathrm{m}^{2}\right)$ & 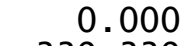 & 0.00 & 0 \\
\hline Total heat flux $\left(\mathrm{kW} / \mathrm{m}^{2}\right)$ & 339.33 & 330.169 & 344.725 \\
\hline Thermal contact conductance $\left(\mathrm{W} / \mathrm{m}^{2} \cdot \mathrm{C}\right)$ & 2454 & 2432.186 & 2473.72 \\
\hline Effective gas gap $(\mu \mathrm{m})$ & 73.95 & 12 & 74.2 \\
\hline Contact therma1 jump distance $(\mu \mathrm{m})$ & 1.176 & 1.17 & 1. \\
\hline Target thermal jump distance $(\mu \mathrm{m})$ & 1.089 & 1.084 & 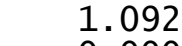 \\
\hline Effective contact pressure (MPa) & 0.0 & 0.000 & \\
\hline Pressure index & 13.5 & 13.534 & 13. \\
\hline $\begin{array}{l}\text { Gas thermal con } \\
\text { solid spot cond }\end{array}$ & 0.1 & 0.187 & 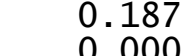 \\
\hline Gas gap conductanc & 2453 & 2432.189 & 2473.11 \\
\hline
\end{tabular}

Contact status codes:

$0=$ open/no heat transfer, $1=$ near-field contact

$2=$ closed and sliding, $\quad 3=$ closed and sticking 
$9.30 \mathrm{~mm}$ holder diameter (tensile design)

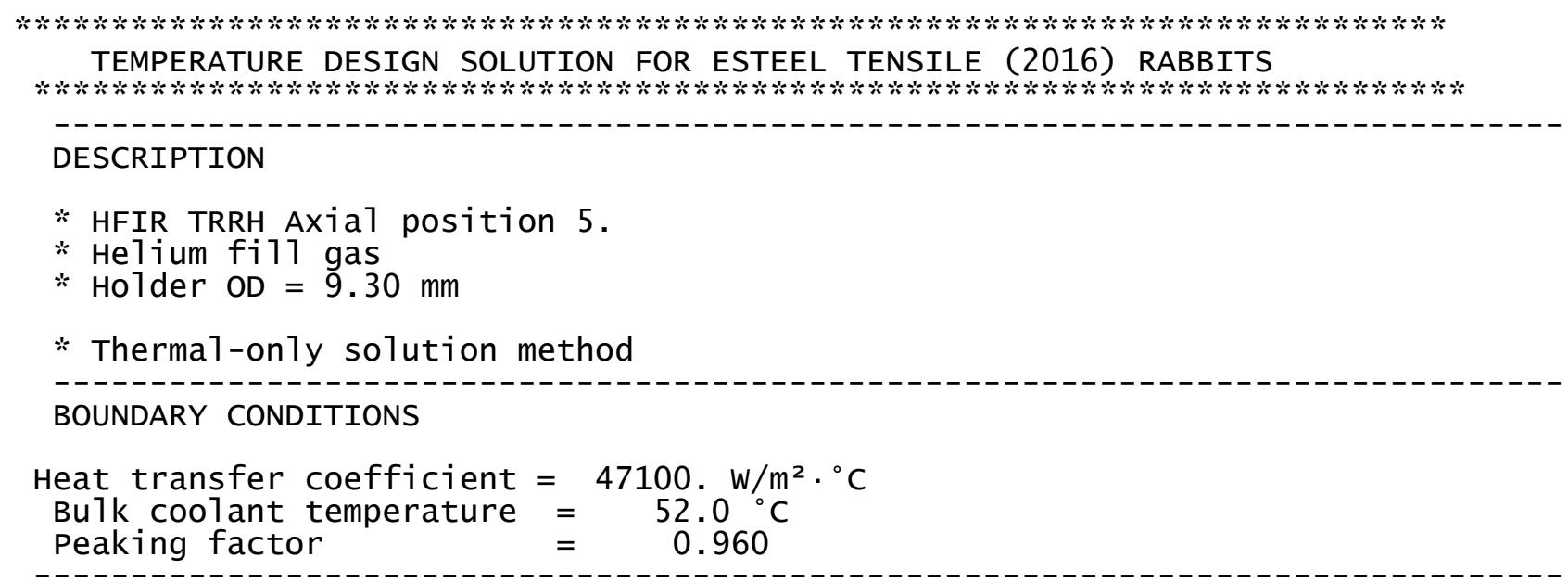

HEAT GENERATION

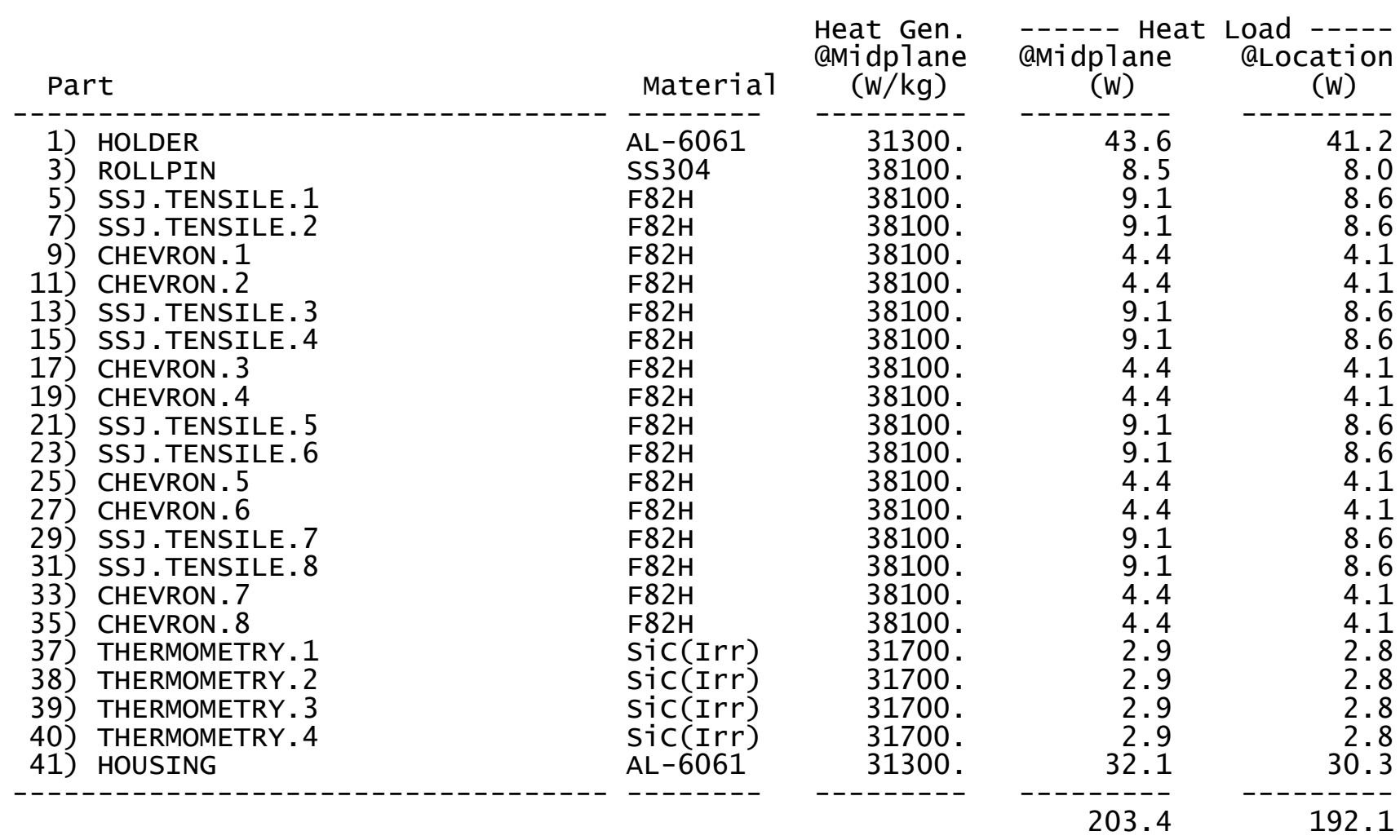

CAPSULE TEMPERATURE SUMMARY

\begin{tabular}{|c|c|c|c|c|c|c|c|}
\hline Name & & Material & Tavg & Tmin & Tmax & Т. 025 & T. 975 \\
\hline $\begin{array}{r}\text { 1) } \\
3 \text { ) } \\
5) \\
7) \\
9) \\
\text { 11) } \\
\text { 13) }\end{array}$ & $\begin{array}{l}\text { HOLDER } \\
\text { ROLLPIN } \\
\text { SSJ.TENSILE. } 1 \\
\text { SSJ.TENSILE. } 2 \\
\text { CHEVRON. } 1 \\
\text { CHEVRON. } 2 \\
\text { SSJ.TENSILE. } 3\end{array}$ & $\begin{array}{l}\text { AL-6061 } \\
\text { SS304 } \\
\text { F82H } \\
\text { F82H } \\
\text { F82H } \\
\text { F82H } \\
\text { F82H }\end{array}$ & $\begin{array}{l}227 . \\
319 . \\
268 . \\
254 . \\
256 . \\
267 . \\
268 .\end{array}$ & $\begin{array}{l}223 . \\
313 \\
247 \\
242 \\
248 \\
254 . \\
247 .\end{array}$ & $\begin{array}{l}231 . \\
330 . \\
280 . \\
263 . \\
263 . \\
275 . \\
279 .\end{array}$ & $\begin{array}{l}224 . \\
314 . \\
252 . \\
245 . \\
251 . \\
261 . \\
252 .\end{array}$ & $\begin{array}{l}230 . \\
327 . \\
278 . \\
260 . \\
261 . \\
273 . \\
277 .\end{array}$ \\
\hline
\end{tabular}


15) SSJ .TENSILE. 4

17) CHEVRON. 3

19) CHEVRON. 4

21) SSJ.TENSILE. 5

23) SSJ .TENSILE.6

25) CHEVRON. 5

27) CHEVRON. 6

29) SSJ.TENSILE.7

31) SSJ.TENSILE. 8

33) CHEVRON.7

35) CHEVRON. 8

37) THERMOMETRY.1

38) THERMOMETRY. 2

39) THERMOMETRY .3

40) THERMOMETRY.4

41) HOUSING
$\mathrm{F} 82 \mathrm{H}$

$\mathrm{F} 82 \mathrm{H}$

$\mathrm{F} 82 \mathrm{H}$

$\mathrm{F} 82 \mathrm{H}$

$\mathrm{F} 82 \mathrm{H}$

$\mathrm{F} 82 \mathrm{H}$

$\mathrm{F} 82 \mathrm{H}$

$\mathrm{F} 82 \mathrm{H}$

$\mathrm{F} 82 \mathrm{H}$

$\mathrm{F} 82 \mathrm{H}$

$\mathrm{F} 82 \mathrm{H}$

$\operatorname{SiC}(\operatorname{Ir} r)$

SiC(Irr)

SiC(Irr)

SiC(Irr)
253. 242. 262.

267.250 .263 .261 .261$.

268. 247. 279. 253. 278 .

254. 241. 262. 245. 260.

256. 248. 263. 251. 261.

267. 255. 274. 260. 272.

268. 247. 279. 252. 278 .

253. 243. 263. 245. 260 .

256. 247. 263. 251. 261.

266. 255. 274. 260. 272 .

272. 236. 298. 249. 288.

272. 235. 298. 248. 288.

273. 235. 301. 248. 289.

273. 237. 301. 248. 289.

PROPERTY SUMMARY AT THE AVERAGE PART TEMPERATURE

Name

1) HOLDER

3) ROLLPIN

5) SSJ.TENSILE.1

7) SSJ.TENSILE.2

9) CHEVRON. 1

11) CHEVRON. 2

13) SSJ.TENSILE. 3

15) SSJ.TENSILE.4

17) CHEVRON. 3

19) CHEVRON. 4

21) SSJ.TENSILE. 5

23) SSJ.TENSILE.6

25) CHEVRON. 5

27) CHEVRON. 6

29) SSJ .TENSILE. 7

31) SSJ.TENSILE.8

33) CHEVRON. 7

35) CHEVRON. 8

37) THERMOMETRY.1

38) THERMOMETRY.2

39) THERMOMETRY. 3

40) THERMOMETRY.4

41) HOUSING

\begin{tabular}{l} 
Material \\
\hline AL-6061 \\
SS304 \\
F82H \\
F82H \\
F82H \\
F82H \\
F82H \\
F82H \\
F82H \\
F82H \\
F82H \\
F82H \\
F82H \\
F82H \\
F82H \\
F82H \\
F82H \\
F82H \\
SiC(Irr) \\
SiC(Irr) \\
SiC(Irr) \\
SiC(Irr) \\
AL-6061
\end{tabular}

AL-6061
Thermat

$\left(\mathrm{W} / \mathrm{m} \cdot{ }^{\circ} \mathrm{C}\right)$ 176.000

19.684

33.589

33.513

33.525

33.585

33.588

33.513

33.525

33.582

33.590

33.514

33.526

33.583

33.588

33.512

33.526

33.580

6.074

6.074

6.074

6.074

166.651
Thermal

Exp. Coeff: Emis $\left(\mu \mathrm{m} / \mathrm{m} \cdot{ }^{\circ} \mathrm{C}\right) \quad(---)$ $24.83 \quad 0.053$ $0.00 \quad 0.143$

$11.14 \quad 0.143$

$11.11 \quad 0.143$

$11.12 \quad 0.143$

$11.13 \quad 0.143$

$11.14 \quad 0.143$

$11.11 \quad 0.143$

$11.12 \quad 0.143$

$11.13 \quad 0.143$

$11.14 \quad 0.143$

$11.11 \quad 0.143$

$11.12 \quad 0.143$

$11.13 \quad 0.143$

$11.14 \quad 0.143$

$11.11 \quad 0.143$

$11.12 \quad 0.143$

$11.13 \quad 0.143$

$\begin{array}{ll}3.18 & 0.900\end{array}$

$3.18 \quad 0.900$

$3.18 \quad 0.900$

$3.18 \quad 0.900$

STORED ENERGY SUMMARY AT THE AVERAGE PART TEMPERATURE

Name

1) HOLDER

3) ROLLPIN

5) SSJ.TENSILE.1

7) SSJ.TENSILE. 2

9) CHEVRON.1

11) CHEVRON. 2

13) SSJ.TENSILE. 3

15) SSJ.TENSILE. 4

\begin{tabular}{|c|c|c|c|c|}
\hline aterial & $\begin{array}{r}\text { Mass } \\
(\mathrm{g})\end{array}$ & $\begin{array}{l}\text { Tavg } \\
\left({ }^{\circ} \mathrm{C}\right)\end{array}$ & $\begin{array}{l}\text { Specific } \\
\text { Heat } \\
\left(\mathrm{J} / \mathrm{kg}^{\circ} \mathrm{C}\right)\end{array}$ & $\begin{array}{c}\text { Stored } \\
\text { Energy } \\
(\mathrm{J})\end{array}$ \\
\hline $\begin{array}{l}\text { AL-6061 } \\
\text { SS304 } \\
\text { F82H } \\
\text { F82H } \\
\text { F82H } \\
\text { F82H } \\
\text { F82H } \\
\text { F82H }\end{array}$ & $\begin{array}{l}1.393 \\
0.223 \\
0.238 \\
0.238 \\
0.115 \\
0.115 \\
0.238 \\
0.238\end{array}$ & $\begin{array}{l}22 \\
31 \\
26 \\
25 \\
25 \\
26 \\
26 \\
25\end{array}$ & $\begin{array}{l}996 . \\
389 . \\
537 . \\
533 . \\
534 . \\
537 . \\
537 .\end{array}$ & $\begin{array}{r}287 . \\
26 . \\
32 . \\
30 . \\
14 .\end{array}$ \\
\hline
\end{tabular}


17) CHEVRON. 3

19) CHEVRON. 4

21) SSJ.TENSILE.5

23) SSJ .TENSILE.6

25) CHEVRON. 5

27) CHEVRON. 6

29) SSJ.TENSILE.7

31) SSJ.TENSILE.8

33) CHEVRON.7

35) CHEVRON. 8

37) THERMOMETRY.1

38) THERMOMETRY.2

39) THERMOMETRY. 3

40) THERMOMETRY. 4

41) HOUSING

$\begin{array}{lrrrr}\text { F82H } & 0.115 & 256 . & 534 . & 14 . \\ \text { F82H } & 0.115 & 267 . & 537 . & 15 . \\ \text { F82H } & 0.238 & 268 . & 537 . & 32 . \\ \text { F82H } & 0.238 & 254 . & 533 . & 30 . \\ \text { F82H } & 0.115 & 256 . & 534 . & 14 . \\ \text { F82H } & 0.115 & 267 . & 537 . & 15 . \\ \text { F82H } & 0.238 & 268 . & 537 . & 32 . \\ \text { F82H } & 0.238 & 253 . & 533 . & 30 . \\ \text { F82H } & 0.115 & 256 . & 534 . & 14 . \\ \text { F82H } & 0.115 & 266 . & 537 . & 15 . \\ \text { SiC(Irr) } & 0.092 & 272 . & 998 . & 23 . \\ \text { SiC(Irr) } & 0.092 & 272 . & 998 . & 23 . \\ \text { SiC(Irr) } & 0.092 & 273 . & 998 . & 23 . \\ \text { SiC(Irr) } & 0.092 & 273 . & 998 . & 23 . \\ \text { AL-6061 } & 1.026 & 60 . & 884 . & 36 . \\ & ---1 .-0 & & & ---1 \\ & 5.832 & & & 806 .\end{array}$

RADIAL DIMENSIONS AND GAP SUMMARY FOR THE CORE-HOUSING GAP

CONTACT SUMMARY FOR CONTACT ID 83: Frictionless - HOLDER TO HOUSING

Contact surface material:

Target surface material:

Interstitial gas:

Effective surface roughness:

Effective asperity slope:

Effective microhardness:

$$
\begin{aligned}
& \text { AL-6061 } \\
& \text { AL-6061 } \\
& \text { He1 1 um } \\
& 2.263 \mu \mathrm{m} \\
& 0.214 \mathrm{rad} \\
& 1.220 \mathrm{GPa}
\end{aligned}
$$

\begin{tabular}{|c|c|c|c|}
\hline & Average & Minimum & Maximum \\
\hline direct results & & & \\
\hline $\begin{array}{l}\text { Contact status } \\
\text { contact temperature }\left({ }^{\circ} \mathrm{C}\right)\end{array}$ & $\begin{array}{r}1.000 \\
27541\end{array}$ & 221.000 & $\begin{array}{r}1.000 \\
227.132\end{array}$ \\
\hline Target temperature $\left({ }^{\circ} \mathrm{C}\right)$ & $\begin{array}{r}223.041 \\
60.664\end{array}$ & $\begin{array}{r}226.070 \\
60.060\end{array}$ & 60.786 \\
\hline Geometric gas gap $(\mu \mathrm{m})$ & 109.967 & 109.646 & 110.248 \\
\hline Contact pressure (MPa) & 0.000 & 0.000 & 0.000 \\
\hline Gap conduction heat f1ux $\left(\mathrm{kW} / \mathrm{m}^{2}\right)$ & 339.249 & 330.888 & 343.630 \\
\hline Radiation heat flux $\left(\mathrm{kW} / \mathrm{m}^{2}\right)$ & 0.000 & 0.000 & 0.000 \\
\hline Contact conduction heat flux $\left(\mathrm{kW} / \mathrm{m}^{2}\right)$ & 0.000 & 0.000 & 0.000 \\
\hline Total heat flux $\left(\mathrm{kW} / \mathrm{m}^{2}\right)$ & 339.249 & 330.8 & 343.63 \\
\hline Therma 1 contact conductance $\left(\mathrm{W} / \mathrm{m}^{2} \cdot \mathrm{C}\right)$ & 2056.30 & 2039.47 & 2067.40 \\
\hline Effective gas gap ( $\mu \mathrm{m})$ & 90.8 & 90. & 91 \\
\hline Contact thermal jump distance $(\mu \mathrm{m})$ & 1.241 & & \\
\hline Target thermal jump distance $(\mu \mathrm{m})$ & 1.134 & 1.129 & 1.137 \\
\hline Effective contact pressure (MPa) & & 0.000 & 000 \\
\hline Pressure index & 13.534 & 13.534 & 13.5 \\
\hline Gas thermal conductivity $\left(\mathrm{W} / \mathrm{m} \cdot{ }^{\circ} \mathrm{C}\right)$ & & 0.191 & 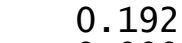 \\
\hline Solid spot conductance $\left(\mathrm{W} / \mathrm{m}^{2}\right.$ & 0.000 & 0.000 & 0.000 \\
\hline Gas gap conductance & 2056.597 & 2039.564 & 2067.67 \\
\hline
\end{tabular}

Contact status codes:

$0=$ open/no heat transfer, $1=$ near-field contact $2=$ closed and sliding, $\quad 3=$ closed and sticking 
$9.25 \mathrm{~mm}$ holder diameter (tensile design)

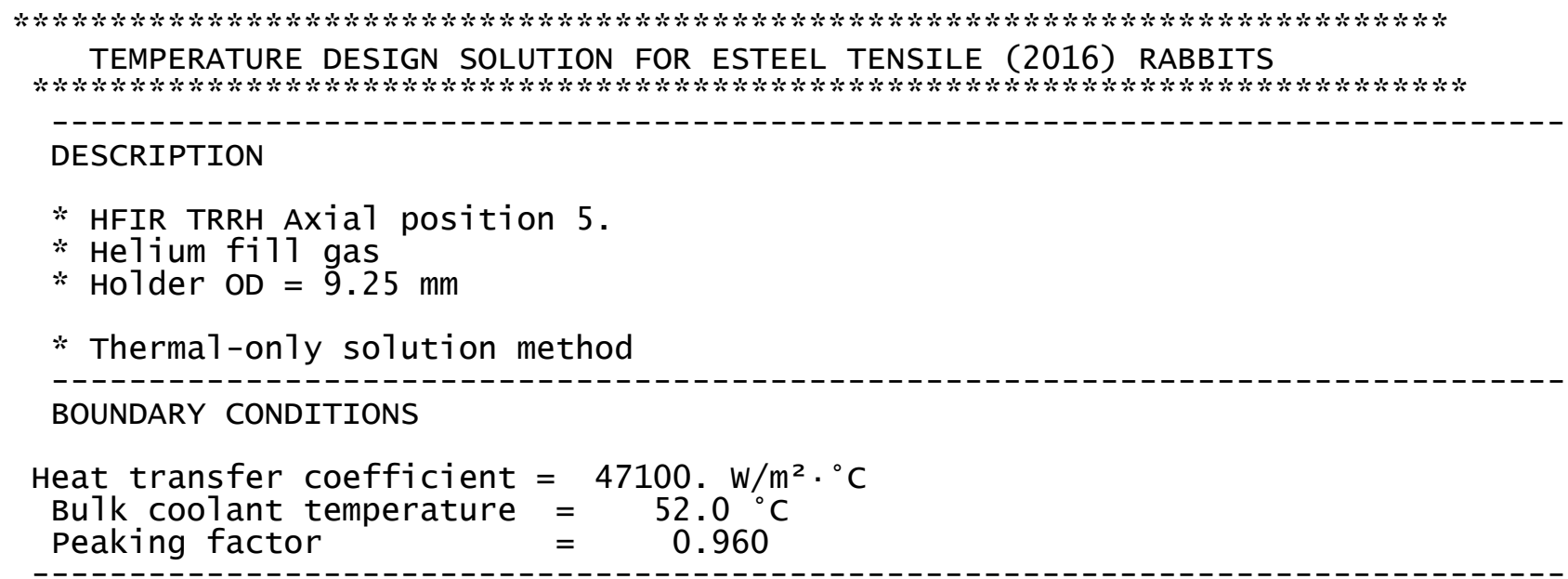

HEAT GENERATION

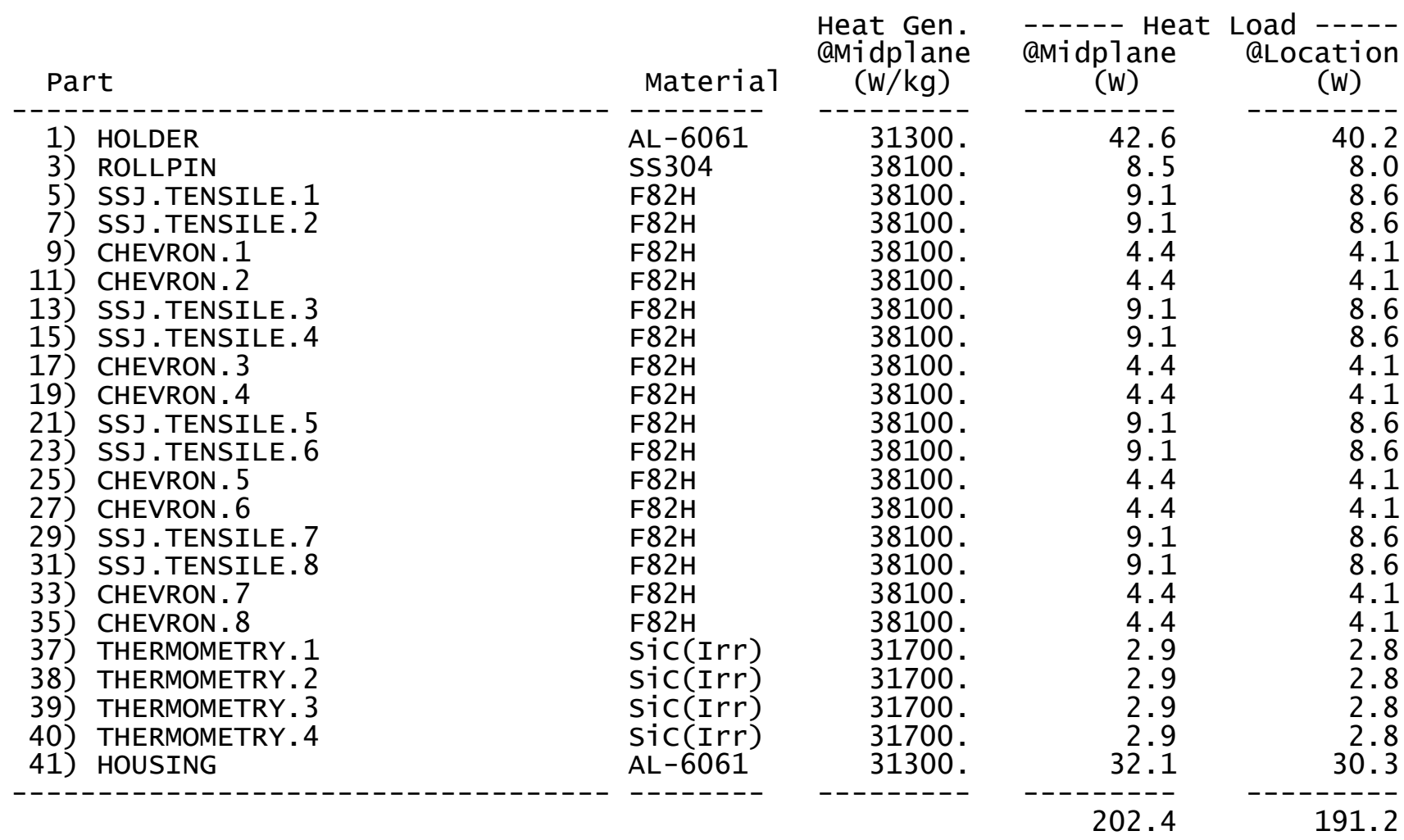

CAPSULE TEMPERATURE SUMMARY

\begin{tabular}{|c|c|c|c|c|c|c|c|}
\hline Name & & Material & Tavg & Tmin & Tmax & T. 025 & Т. 975 \\
\hline $\begin{array}{r}\text { 1) } \\
3) \\
5) \\
7) \\
9) \\
11) \\
13)\end{array}$ & $\begin{array}{l}\text { HOLDER } \\
\text { ROLLPIN } \\
\text { SSJ.TENSILE. } 1 \\
\text { SSJ.TENSILE. } 2 \\
\text { CHEVRON. } 1 \\
\text { CHEVRON. } 2 \\
\text { SSJ.TENSILE. } 3\end{array}$ & $\begin{array}{l}\text { AL-6061 } \\
\text { SS304 } \\
\text { F82H } \\
\text { F82H } \\
\text { F82H } \\
\text { F82H } \\
\text { F82H }\end{array}$ & $\begin{array}{l}259 . \\
350 . \\
299 . \\
285 . \\
287 . \\
298 . \\
299 .\end{array}$ & $\begin{array}{l}255 . \\
344 . \\
278 . \\
274 . \\
279 . \\
287 . \\
279 .\end{array}$ & $\begin{array}{l}263 . \\
361 . \\
311 . \\
294 . \\
294 . \\
306 . \\
311 .\end{array}$ & $\begin{array}{l}256 . \\
346 . \\
284 . \\
277 \\
283 . \\
292 \\
284 .\end{array}$ & $\begin{array}{l}262 . \\
358 . \\
309 . \\
292 . \\
292 . \\
304 . \\
309 .\end{array}$ \\
\hline
\end{tabular}


15) SSJ .TENSILE. 4

17) CHEVRON. 3

19) CHEVRON. 4

21) SSJ.TENSILE. 5

23) SSJ .TENSILE.6

25) CHEVRON. 5

27) CHEVRON. 6

29) SSJ.TENSILE.7

31) SSJ .TENSILE. 8

33) CHEVRON.7

35) CHEVRON. 8

37) THERMOMETRY.1

38) THERMOMETRY. 2

39) THERMOMETRY .3

40) THERMOMETRY.4

41) HOUSING
$\mathrm{F} 82 \mathrm{H}$

$\mathrm{F} 82 \mathrm{H}$

$\mathrm{F} 82 \mathrm{H}$

$\mathrm{F} 82 \mathrm{H}$

$\mathrm{F} 82 \mathrm{H}$

$\mathrm{F} 82 \mathrm{H}$

$\mathrm{F} 82 \mathrm{H}$

$\mathrm{F} 82 \mathrm{H}$

$\mathrm{F} 82 \mathrm{H}$

$\mathrm{F} 82 \mathrm{H}$

$\mathrm{F} 82 \mathrm{H}$

$\operatorname{SiC}(\operatorname{Ir} r)$

SiC(Irr)

SiC(Irr)

Sic(Irr)

AL-6061
285. 273. 293. 277. 291.

287. 260. 295. 281. 293.

299. 286. 306. 292. 304.

300. 279. 311. 284. 309.

285. 274. 294. 277. 292.

288. 279. 295. 283. 293.

298. 286. 306. 292. 304.

300. 280. 311. 284. 310.

286. 274. 294. 277. 292.

288. 280. 295. 283. 293.

299. 286. 307. 292. 305.

304. 270. 330. 280. 320.

304. 270. 329. 281. 320.

304. 268. 333. 280. 320 .

305. 269. 333. 281. 321.

PROPERTY SUMMARY AT THE AVERAGE PART TEMPERATURE

Name

1) HOLDER

3) ROLLPIN

5) SSJ.TENSILE.1

7) SSJ.TENSILE.2

9) CHEVRON.1

11) CHEVRON. 2

13) SSJ.TENSILE. 3

15) SSJ.TENSILE. 4

17) CHEVRON. 3

19) CHEVRON. 4

21) SSJ.TENSILE. 5

23) SSJ.TENSILE. 6

25) CHEVRON. 5

27) CHEVRON. 6

29) SSJ .TENSILE. 7

31) SSJ.TENSILE.8

33) CHEVRON. 7

35) CHEVRON. 8

37) THERMOMETRY.1

38) THERMOMETRY.2

39) THERMOMETRY. 3

40) THERMOMETRY.4

41) HOUSING

\begin{tabular}{l} 
Material \\
\hline AL-6061 \\
SS304 \\
F82H \\
F82H \\
F82H \\
F82H \\
F82H \\
F82H \\
F82H \\
F82H \\
F82H \\
F82H \\
F82H \\
F82H \\
F82H \\
F82H \\
F82H \\
F82H \\
SiC(Irr) \\
SiC(Irr) \\
SiC(Irr) \\
SiC(Irr)
\end{tabular}

AL-6061

\section{Thermat \\ cond.}

$\left(\mathrm{W} / \mathrm{m} \cdot{ }^{\circ} \mathrm{C}\right)$

176.000

20.119

33.756

33.681

33.693

33.751

33.755

33.680

33.691

33.752

33.758

33.683

33.695

33.751

33.758

33.683

33.695

33.754

6.062

6.062

6.062

6.062

166.647
Thermal

Exp. Coeff. Emis $\left(\mu \mathrm{m} / \mathrm{m} \cdot{ }^{\circ} \mathrm{C}\right)(---)$

$25.09 \quad 0.055$

$0.00 \quad 0.143$

$11.19 \quad 0.143$

$11.17 \quad 0.143$

$11.17 \quad 0.143$

$11.19 \quad 0.143$

$11.19 \quad 0.143$

$11.17 \quad 0.143$

$11.17 \quad 0.143$

$11.19 \quad 0.143$

$11.19 \quad 0.143$

$11.17 \quad 0.143$

$11.17 \quad 0.143$

$11.19 \quad 0.143$

$11.19 \quad 0.143$

$11.17 \quad 0.143$

$11.17 \quad 0.143$

$11.19 \quad 0.143$

$3.28 \quad 0.900$

$3.28 \quad 0.900$

$3.28 \quad 0.900$

$3.28 \quad 0.900$

STORED ENERGY SUMMARY AT THE AVERAGE PART TEMPERATURE

Name

1) HOLDER

3) ROLLPIN

5) SSJ.TENSILE.1

7) SSJ.TENSILE. 2

9) CHEVRON.1

11) CHEVRON. 2

13) SSJ.TENSILE. 3

15) SSJ.TENSILE. 4

\begin{tabular}{|c|c|c|c|c|}
\hline aterial & $\begin{array}{r}\text { Mass } \\
(\mathrm{g})\end{array}$ & $\begin{array}{l}\text { Tavg } \\
\left({ }^{\circ} \mathrm{C}\right)\end{array}$ & $\begin{array}{c}\text { Specific } \\
\text { Heat } \\
\left(\mathrm{J} / \mathrm{kg}^{\circ} \mathrm{C}\right)\end{array}$ & $\begin{array}{l}\text { Stored } \\
\text { Energy } \\
(\mathrm{J})\end{array}$ \\
\hline $\begin{array}{l}\mathrm{L}-6061 \\
\mathrm{~S} 304 \\
82 \mathrm{H} \\
82 \mathrm{H} \\
82 \mathrm{H} \\
82 \mathrm{H} \\
82 \mathrm{H}\end{array}$ & $\begin{array}{l}1.361 \\
0.223 \\
0.238 \\
0.238 \\
0.115 \\
0.115 \\
0.238 \\
0.238\end{array}$ & $\begin{array}{l}259 . \\
350 . \\
299 . \\
285 . \\
287 . \\
298 . \\
299 . \\
285 .\end{array}$ & $\begin{array}{r}1010 . \\
392 . \\
548 . \\
542 . \\
543 . \\
547 . \\
548 . \\
542 .\end{array}$ & $\begin{array}{r}329 . \\
29 . \\
36 . \\
34 . \\
17 . \\
17 . \\
36 . \\
34 .\end{array}$ \\
\hline
\end{tabular}


17) CHEVRON. 3

19) CHEVRON. 4

21) SSJ.TENSILE.5

23) SSJ .TENSILE.6

25) CHEVRON. 5

27) CHEVRON. 6

29) SSJ.TENSILE.7

31) SSJ.TENSILE.8

33) CHEVRON.7

35) CHEVRON. 8

37) THERMOMETRY.1

38) THERMOMETRY.2

39) THERMOMETRY. 3

40) THERMOMETRY. 4

41) HOUSING

$\begin{array}{lrrrr}\text { F82H } & 0.115 & 287 . & 543 . & 17 . \\ \text { F82H } & 0.115 & 299 . & 548 . & 17 . \\ \text { F82H } & 0.238 & 300 . & 548 . & 36 . \\ \text { F82H } & 0.238 & 285 . & 542 . & 34 . \\ \text { F82H } & 0.115 & 288 . & 543 . & 17 . \\ \text { F82H } & 0.115 & 298 . & 547 . & 17 . \\ \text { F82H } & 0.238 & 300 . & 548 . & 36 . \\ \text { F82H } & 0.238 & 286 . & 542 . & 34 . \\ \text { F82H } & 0.115 & 288 . & 543 . & 17 . \\ \text { F82H } & 0.115 & 299 . & 548 . & 18 . \\ \text { SiC(Irr) } & 0.092 & 304 . & 1021 . & 27 . \\ \text { SiC(Irr) } & 0.092 & 304 . & 1021 . & 27 . \\ \text { SiC(Irr) } & 0.092 & 304 . & 1021 . & 27 . \\ \text { SiC(Irr) } & 0.092 & 305 . & 1021 . & 27 . \\ \text { AL-6061 } & 1.026 & 60 . & 884 . & 36 . \\ & ----10- & & & -1 . \\ & 5.800 & & & 920 .\end{array}$

RADIAL DIMENSIONS AND GAP SUMMARY FOR THE CORE-HOUSING GAP

CONTACT SUMMARY FOR CONTACT ID 83: Frictionless - HOLDER TO HOUSING

Contact surface material:

Target surface material:

Interstitial gas:

Effective surface roughness:

Effective asperity slope:

Effective microhardness:

$$
\begin{aligned}
& \text { AL-6061 } \\
& \text { AL-6061 } \\
& \text { He1 1 um } \\
& 2.263 \mu \mathrm{m} \\
& 0.214 \mathrm{rad} \\
& 1.220 \mathrm{GPa}
\end{aligned}
$$

\begin{tabular}{|c|c|c|c|}
\hline & Average & Minimum & Maximum \\
\hline direct results & & & \\
\hline $\begin{array}{l}\text { Contact status } \\
\text { Contact temperature }\left({ }^{\circ} \mathrm{C}\right)\end{array}$ & $\begin{array}{r}1.000 \\
257.661\end{array}$ & $\begin{array}{r}1.000 \\
254.654\end{array}$ & $\begin{array}{r}1.000 \\
259.210\end{array}$ \\
\hline Target temperature $\left({ }^{\circ} \mathrm{C}\right)$ & 60.621 & $\begin{array}{r}234.034 \\
60.036\end{array}$ & $\begin{array}{r}259.210 \\
60.759\end{array}$ \\
\hline Geometric gas gap $(\mu \mathrm{m})$ & 134.963 & 134.404 & 135.209 \\
\hline Contact pressure (MPa) & 0.000 & 0.000 & 0.000 \\
\hline Gap conduction heat f1ux $\left(\mathrm{kW} / \mathrm{m}^{2}\right)$ & 339.112 & 331.735 & 343.136 \\
\hline Radiation heat flux $\left(\mathrm{kW} / \mathrm{m}^{2}\right)$ & 0.000 & 0.000 & 0.000 \\
\hline Contact conduction heat flux $\left(\mathrm{kW} / \mathrm{m}^{2}\right)$ & 0.000 & 0.000 & 0.00 \\
\hline Total heat flux $\left(\mathrm{kW} / \mathrm{m}^{2}\right)$ & 339.112 & 331.73 & 343.13 \\
\hline Therma 1 contact conductance $\left(\mathrm{W} / \mathrm{m}^{2} \cdot \mathrm{C}\right)$ & 1721.007 & 1708.361 & 1732.5 \\
\hline 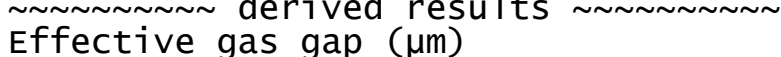 & 111 & 111.277 & 112. \\
\hline Contact therma1 jump distance $(\mu \mathrm{m})$ & 12 & 1.312 & \\
\hline Target thermal jump distance $(\mu \mathrm{m})$ & 1.188 & 1.182 & 1.1 \\
\hline Effective contact pressure (MPa) & & 0.000 & \\
\hline Pressure index & 13.534 & 13.534 & 13.5 \\
\hline Gas thermal conduc & 0.1 & 0.1 & 0 . \\
\hline Solid spot conductan & 0.000 & 0.000 & 0.000 \\
\hline Gas gap conductance & 1720 & 1708.006 & 1731.73 \\
\hline
\end{tabular}

Contact status codes:

$0=$ open/no heat transfer, $1=$ near-field contact $2=$ closed and sliding, $\quad 3=$ closed and sticking 
$9.20 \mathrm{~mm}$ holder diameter (tensile design)

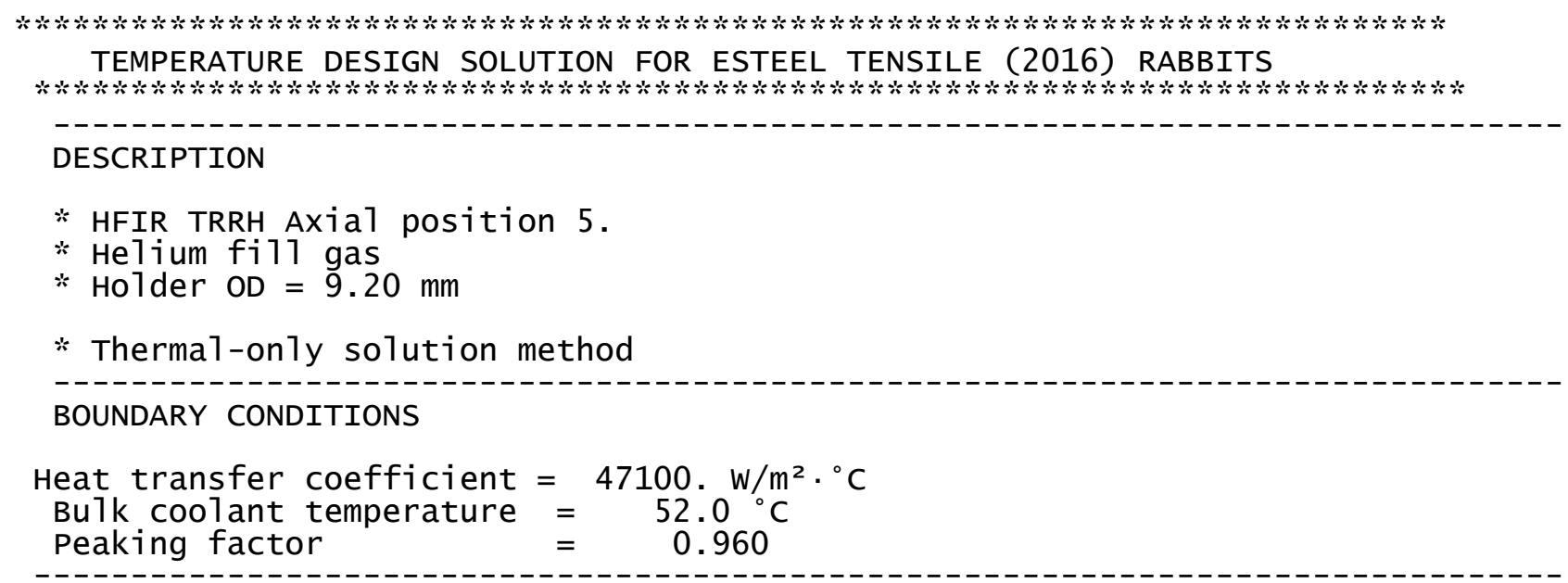

HEAT GENERATION

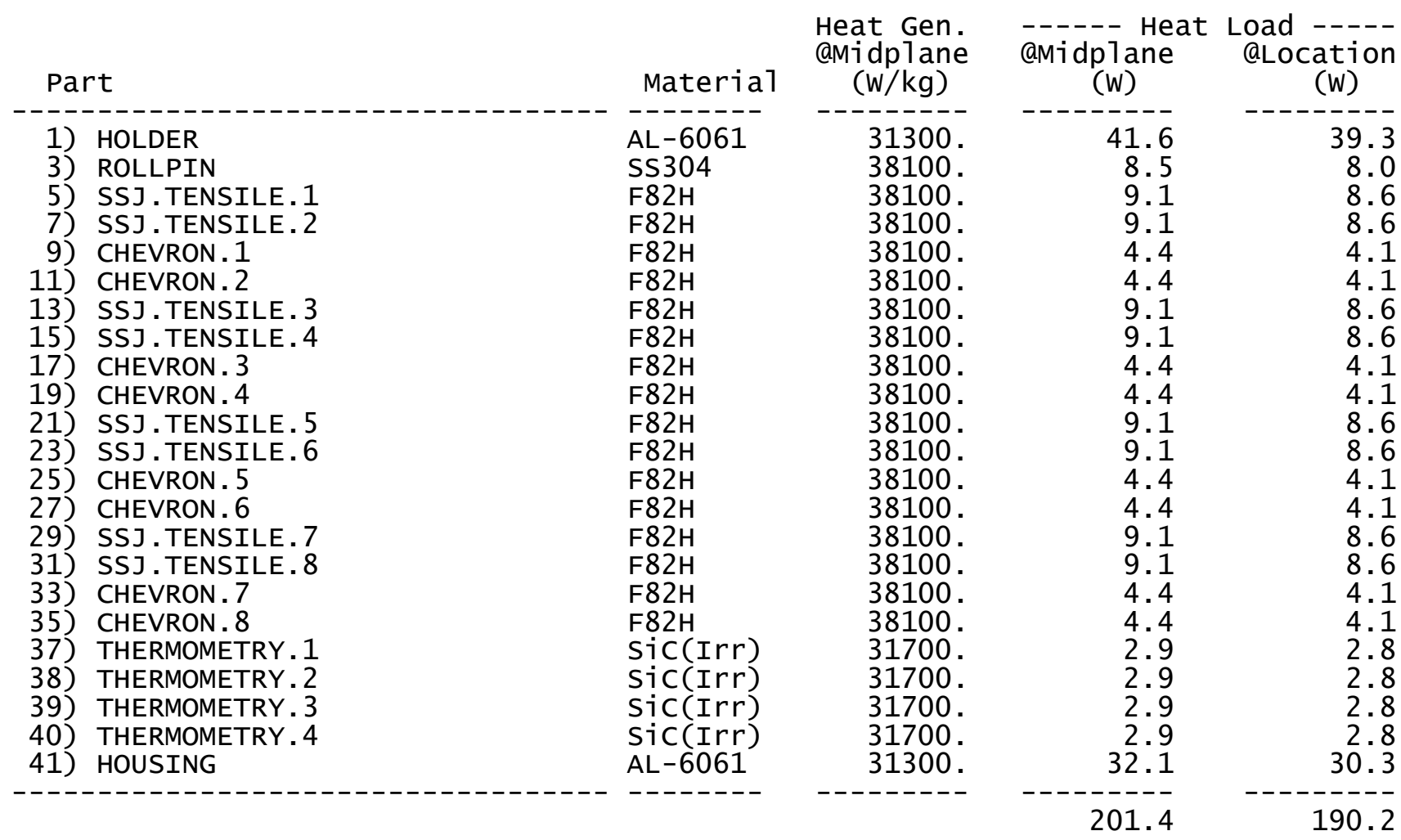

CAPSULE TEMPERATURE SUMMARY

\begin{tabular}{|c|c|c|c|c|c|c|c|}
\hline Name & & Material & Tavg & Tmin & Tmax & T. 025 & T. 975 \\
\hline $\begin{array}{r}\text { 1) } \\
3 \text { ) } \\
5) \\
7) \\
9) \\
\text { 11) } \\
\text { 13) }\end{array}$ & $\begin{array}{l}\text { HOLDER } \\
\text { ROLLPIN } \\
\text { SSJ.TENSILE. } 1 \\
\text { SSJ.TENSILE. } 2 \\
\text { CHEVRON. } 1 \\
\text { CHEVRON. } 2 \\
\text { SSJ.TENSILE. } 3\end{array}$ & $\begin{array}{l}\text { AL-6061 } \\
\text { SS304 } \\
\text { F82H } \\
\text { F82H } \\
\text { F82H } \\
\text { F82H } \\
\text { F82H }\end{array}$ & $\begin{array}{l}290 . \\
380 . \\
330 . \\
316 . \\
318 . \\
329 . \\
330 .\end{array}$ & $\begin{array}{l}285 . \\
374 . \\
309 . \\
305 . \\
310 . \\
316 . \\
309\end{array}$ & $\begin{array}{l}293 . \\
390 \\
341 \\
324 \\
325 \\
337 \\
341\end{array}$ & $\begin{array}{l}286 . \\
375 \\
314 . \\
308 \\
313 . \\
322 . \\
315\end{array}$ & $\begin{array}{l}292 . \\
388 . \\
339 . \\
322 . \\
323 . \\
334 . \\
339 .\end{array}$ \\
\hline
\end{tabular}


15) SSJ .TENSILE. 4

17) CHEVRON. 3

19) CHEVRON. 4

21) SSJ.TENSILE. 5

23) SSJ .TENSILE.6

25) CHEVRON. 5

27) CHEVRON. 6

29) SSJ.TENSILE.7

31) SSJ.TENSILE. 8

33) CHEVRON.7

35) CHEVRON. 8

37) THERMOMETRY.1

38) THERMOMETRY. 2

39) THERMOMETRY .3

40) THERMOMETRY.4

41) HOUSING
$\mathrm{F} 82 \mathrm{H}$

$\mathrm{F} 82 \mathrm{H}$

$\mathrm{F} 82 \mathrm{H}$

$\mathrm{F} 82 \mathrm{H}$

$\mathrm{F} 82 \mathrm{H}$

$\mathrm{F} 82 \mathrm{H}$

$\mathrm{F} 82 \mathrm{H}$

$\mathrm{F} 82 \mathrm{H}$

$\mathrm{F} 82 \mathrm{H}$

$\mathrm{F} 82 \mathrm{H}$

$\mathrm{F} 82 \mathrm{H}$

$\operatorname{SiC}(\operatorname{Ir} r)$

SiC(Irr)

SiC(Irr)

Sic(Irr)
315. 303. 324. 307. 322.

317. 293. 324. 310. 322.

329. 317. 336. 323. 334.

330. 309. 342. 315. 340 .

316. 305. 325. 308. 322 .

318. 310. 325. 314. 323.

329. 316. 336. 323. 334.

330. 310. 342. 315. 340.

316. 305. 325. 308. 322.

318. 310. 325. 313. 323.

329. 317. 337. 323. 335.

335. 300. 360. 310. 350.

334. 300. 359. 311. 350.

334. 299. 363. 310. 351.

335. 299. 363. 311. 351.

PROPERTY SUMMARY AT THE AVERAGE PART TEMPERATURE

Name

1) HOLDER

3) ROLLPIN

5) SSJ.TENSILE.1

7) SSJ.TENSILE.2

9) CHEVRON.1

11) CHEVRON. 2

13) SSJ.TENSILE. 3

15) SSJ.TENSILE. 4

17) CHEVRON. 3

19) CHEVRON. 4

21) SSJ.TENSILE. 5

23) SSJ.TENSILE. 6

25) CHEVRON. 5

27) CHEVRON. 6

29) SSJ .TENSILE. 7

31) SSJ.TENSILE.8

33) CHEVRON. 7

35) CHEVRON. 8

37) THERMOMETRY.1

38) THERMOMETRY.2

39) THERMOMETRY. 3

40) THERMOMETRY.4

41) HOUSING

\begin{tabular}{l} 
Material \\
\hline AL-6061 \\
SS304 \\
F82H \\
F82H \\
F82H \\
F82H \\
F82H \\
F82H \\
F82H \\
F82H \\
F82H \\
F82H \\
F82H \\
F82H \\
F82H \\
F82H \\
F82H \\
F82H \\
SiC(Irr) \\
SiC(Irr) \\
SiC(Irr) \\
SiC(Irr)
\end{tabular}

AL-6061
Thermal cond.

$\left(\mathrm{W} / \mathrm{m} \cdot{ }^{\circ} \mathrm{C}\right)$ 176.000

20.536

33.752

33.782

33.777

33.754

33.752

33.783

33.780

33.753

33.751

33.782

33.777

33.753

33.751

33.782

33.777

33.752

6.050

6.050

6.050

6.050

166.642
Thermal

Exp. Coeff: Emis $\left(\mu \mathrm{m} / \mathrm{m} \cdot{ }^{\circ} \mathrm{C}\right)(---)$

$25.34 \quad 0.056$

$0.00 \quad 0.143$

$11.34 \quad 0.143$

$11.27 \quad 0.143$

$11.28 \quad 0.143$

$11.33 \quad 0.143$

$11.34 \quad 0.143$

$11.27 \quad 0.143$

$11.27 \quad 0.143$

$11.33 \quad 0.143$

$11.34 \quad 0.143$

$11.27 \quad 0.143$

$11.28 \quad 0.143$

$11.33 \quad 0.143$

$11.34 \quad 0.143$

$11.27 \quad 0.143$

$11.28 \quad 0.143$

$11.34 \quad 0.143$

$\begin{array}{ll}3.37 & 0.900\end{array}$

$\begin{array}{ll}3.37 & 0.900\end{array}$

$\begin{array}{ll}3.37 & 0.900\end{array}$

$\begin{array}{rl}3.37 & 0.900\end{array}$

STORED ENERGY SUMMARY AT THE AVERAGE PART TEMPERATURE

Name

1) HOLDER

3) ROLLPIN

5) SSJ.TENSILE.1

7) SSJ.TENSILE. 2

9) CHEVRON.1

11) CHEVRON. 2

13) SSJ.TENSILE. 3

15) SSJ.TENSILE. 4

\begin{tabular}{|c|c|c|c|c|}
\hline aterial & $\begin{array}{r}\text { Mass } \\
(\mathrm{g})\end{array}$ & $\begin{array}{l}\text { Tavg } \\
\left({ }^{\circ} \mathrm{C}\right)\end{array}$ & $\begin{array}{l}\text { Specific } \\
\text { Heat } \\
\left(\mathrm{J} / \mathrm{kg}^{\circ} \mathrm{C}\right)\end{array}$ & $\begin{array}{c}\text { Stored } \\
\text { Energy } \\
(\mathrm{J})\end{array}$ \\
\hline $\begin{array}{l}\text { AL-6061 } \\
\text { SS304 } \\
\text { F82H } \\
\text { F82H } \\
\text { F82H } \\
\text { F82H } \\
\text { F82H } \\
\text { F82H }\end{array}$ & $\begin{array}{l}1.329 \\
0.223 \\
0.238 \\
0.238 \\
0.115 \\
0.115 \\
0.238 \\
0.238\end{array}$ & $\begin{array}{l}290 . \\
380 . \\
330 . \\
316 . \\
318 . \\
329 .\end{array}$ & $\begin{array}{l}1024 . \\
394 . \\
563 . \\
556 . \\
558 . \\
563 . \\
563 . \\
556 .\end{array}$ & $\begin{array}{r}367 . \\
32 . \\
42 . \\
39 . \\
19 .\end{array}$ \\
\hline
\end{tabular}


17) CHEVRON. 3

19) CHEVRON. 4

21) SSJ.TENSILE.5

23) SSJ .TENSILE.6

25) CHEVRON. 5

27) CHEVRON. 6

29) SSJ.TENSILE. 7

31) SSJ .TENSILE.8

33) CHEVRON. 7

35) CHEVRON .8

37) THERMOMETRY.1

38) THERMOMETRY.2

39) THERMOMETRY. 3

40) THERMOMETRY. 4

41) HOUSING

$\begin{array}{lrrrr}\text { F82H } & 0.115 & 317 . & 557 . & 19 . \\ \text { F82H } & 0.115 & 329 . & 563 . & 20 . \\ \text { F82H } & 0.238 & 330 . & 564 . & 42 . \\ \text { F82H } & 0.238 & 316 . & 556 . & 39 . \\ \text { F82H } & 0.115 & 318 . & 558 . & 19 . \\ \text { F82H } & 0.115 & 329 . & 563 . & 20 . \\ \text { F82H } & 0.238 & 330 . & 564 . & 42 . \\ \text { F82H } & 0.238 & 316 . & 556 . & 39 . \\ \text { F82H } & 0.115 & 318 . & 558 . & 19 . \\ \text { F82H } & 0.115 & 329 . & 563 . & 20 . \\ \text { SiC(Irr) } & 0.092 & 335 . & 1038 . & 30 . \\ \text { SiC(Irr) } & 0.092 & 334 . & 1037 . & 30 . \\ \text { SiC(Irr) } & 0.092 & 334 . & 1037 . & 30 . \\ \text { SiC(Irr) } & 0.092 & 335 . & 1038 . & 30 . \\ \text { AL-6061 } & 1.026 & 60 . & 884 . & 36 . \\ & ---1.0- & & & --1034 .\end{array}$

RADIAL DIMENSIONS AND GAP SUMMARY FOR THE CORE-HOUSING GAP

CONTACT SUMMARY FOR CONTACT ID 83: Frictionless - HOLDER TO HOUSING

Contact surface material:

Target surface material:

Interstitial gas:

Effective surface roughness:

Effective asperity slope:

Effective microhardness:
AL-6061
$\mathrm{AL}-6061$
Helium
$2.263 \mu \mathrm{m}$
$0.214 \mathrm{rad}$
$1.220 \mathrm{GPa}$

\begin{tabular}{|c|c|c|c|}
\hline & Average & Minimum & Maximum \\
\hline 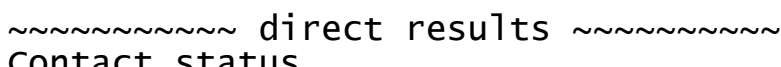 & & & \\
\hline $\begin{array}{l}\text { Contact status } \\
\text { contact temperature }\left({ }^{\circ} \mathrm{C}\right)\end{array}$ & $\begin{array}{r}1.000 \\
288.531\end{array}$ & $\begin{array}{r}1.000 \\
285.365\end{array}$ & $\begin{array}{r}1.000 \\
290.210\end{array}$ \\
\hline Target temperature $\left({ }^{\circ} \mathrm{C}\right)$ & $\begin{array}{r}200.531 \\
60.580\end{array}$ & 59.981 & 60.703 \\
\hline Geometric gas gap $(\mu \mathrm{m})$ & 159.966 & 159.641 & 161.334 \\
\hline Contact pressure (MPa) & 0.000 & 0.000 & 0.000 \\
\hline Gap conduction heat f1ux $\left(\mathrm{kW} / \mathrm{m}^{2}\right)$ & 339.007 & 332.212 & 342.637 \\
\hline Radiation heat flux $\left(\mathrm{kW} / \mathrm{m}^{2}\right)$ & 0.000 & 0.000 & 0.000 \\
\hline Contact conduction heat flux $\left(\mathrm{kW} / \mathrm{m}^{2}\right)$ & 0.000 & 0.000 & 0.000 \\
\hline Total heat flux $\left(\mathrm{kW} / \mathrm{m}^{2}\right)$ & 339.007 & 332. & 342.637 \\
\hline Therma 1 contact conductance $\left(\mathrm{W} / \mathrm{m}^{2} \cdot \mathrm{C}\right)$ & 1487.176 & 1474.32 & 1493.55 \\
\hline Effective gas gap $(\mu \mathrm{m})$ & 133 & 132.7 & 134.477 \\
\hline Contact thermal jump distance $(\mu \mathrm{m})$ & & & 1.400 \\
\hline Target thermal jump distance $(\mu \mathrm{m})$ & 1.2 & 1.23 & 1.2 - -10 \\
\hline Effective contact pressure (MPa) & & 0. & 0.000 \\
\hline Pressure index & 13.5 & 13.5 & 13.534 \\
\hline Gas thermal conductivity $\left(\mathrm{W} / \mathrm{m} \cdot{ }^{\circ} \mathrm{C}\right)$ & & 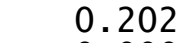 & 02 \\
\hline Solid spot conductance $\left(\mathrm{W} / \mathrm{m}^{2} \cdot \mathrm{C}\right)$ & 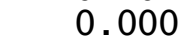 & 0 & 0.000 \\
\hline Gas gap conductance & 1486. & 1474.005 & 1493.240 \\
\hline
\end{tabular}

Contact status codes:

$0=$ open/no heat transfer, $1=$ near-field contact

$2=$ closed and sliding, $\quad 3=c l o s e d$ and sticking 
$9.15 \mathrm{~mm}$ holder diameter (tensile design)

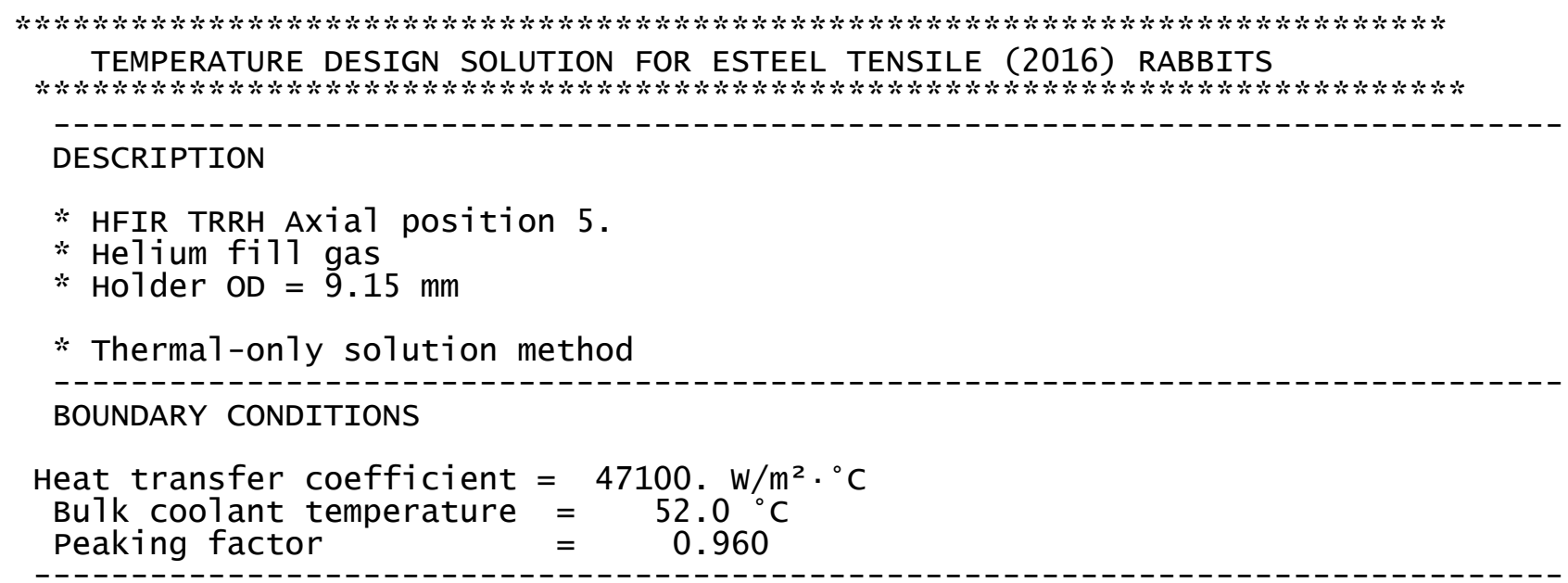

HEAT GENERATION

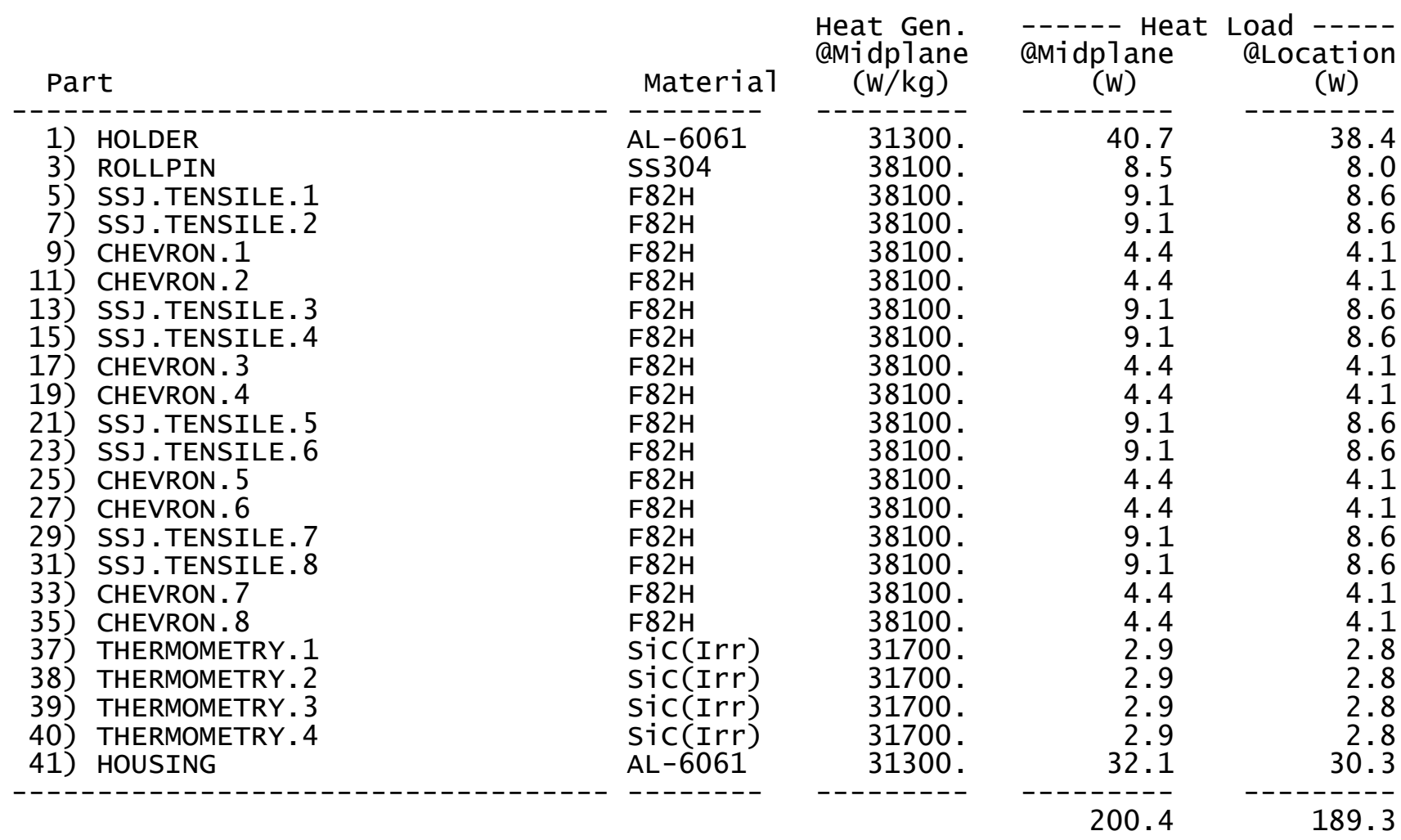

CAPSULE TEMPERATURE SUMMARY

\begin{tabular}{|c|c|c|c|c|c|c|c|}
\hline Name & & Material & Tavg & Tmin & Tmax & T. 025 & T. 975 \\
\hline $\begin{array}{r}1) \\
3) \\
5) \\
7) \\
9) \\
11) \\
13)\end{array}$ & $\begin{array}{l}\text { HOLDER } \\
\text { ROLLPIN } \\
\text { SSJ. TENSILE. } 1 \\
\text { SSJ. TENSILE. } 2 \\
\text { CHEVRON. } 1 \\
\text { CHEVRON. } 2 \\
\text { SSJ. TENSILE. } 3\end{array}$ & $\begin{array}{l}\text { AL-6061 } \\
\text { SS304 } \\
\text { F82H } \\
\text { F82H } \\
\text { F82H } \\
\text { F82H } \\
\text { F82H }\end{array}$ & $\begin{array}{l}320 . \\
409 . \\
360 . \\
346 . \\
347 . \\
359 . \\
358 .\end{array}$ & $\begin{array}{l}315 . \\
403 \\
339 . \\
334 . \\
338 \\
347 \\
339\end{array}$ & $\begin{array}{l}324 . \\
419 . \\
371 \\
355 \\
355 \\
367 \\
370\end{array}$ & $\begin{array}{l}316 . \\
404 . \\
344 . \\
337 . \\
342 . \\
353 . \\
344 .\end{array}$ & $\begin{array}{l}322 . \\
416 . \\
369 . \\
352 . \\
353 . \\
365 . \\
369\end{array}$ \\
\hline
\end{tabular}


15) SSJ .TENSILE. 4

17) CHEVRON. 3

19) CHEVRON. 4

21) SSJ.TENSILE. 5

23) SSJ .TENSILE.6

25) CHEVRON. 5

27) CHEVRON. 6

29) SSJ.TENSILE.7

31) SSJ .TENSILE. 8

33) CHEVRON.7

35) CHEVRON. 8

37) THERMOMETRY.1

38) THERMOMETRY. 2

39) THERMOMETRY .3

40) THERMOMETRY.4

41) HOUSING
$\mathrm{F} 82 \mathrm{H}$

$\mathrm{F} 82 \mathrm{H}$

$\mathrm{F} 82 \mathrm{H}$

$\mathrm{F} 82 \mathrm{H}$

$\mathrm{F} 82 \mathrm{H}$

$\mathrm{F} 82 \mathrm{H}$

$\mathrm{F} 82 \mathrm{H}$

$\mathrm{F} 82 \mathrm{H}$

$\mathrm{F} 82 \mathrm{H}$

$\mathrm{F} 82 \mathrm{H}$

$\mathrm{F} 82 \mathrm{H}$

$\operatorname{SiC}(\operatorname{Ir} r)$

SiC(Irr)

SiC(Irr)

SiC(Irr)
344. 333.353.

341. 317. 350.

358.346 .366$.

360 . 339. 371.

346.335 .354$.

348. 339. 355.

359. 348. 366.

360. 340. 371 .

346. 334. 355.

347. 326. 355.

359. 347. 367.

364 . 329. 390.

364. 329. 389.

363. 329. 392.

365. 330. 393.
337. 351 .

331. $\quad 347$.

351. 364.

345. 369 .

338. 351.

343. 353 .

353.365.

344. $\quad 370$.

337.352.

341. 352 .

352. 365 .

341 . $\quad 380$.

340.380.

339. 380 .

341. 381 .

PROPERTY SUMMARY AT THE AVERAGE PART TEMPERATURE

Name

1) HOLDER

3) ROLLPIN

5) SSJ.TENSILE.1

7) SSJ.TENSILE.2

9) CHEVRON.1

11) CHEVRON. 2

13) SSJ.TENSILE. 3

15) SSJ.TENSILE. 4

17) CHEVRON. 3

19) CHEVRON. 4

21) SSJ.TENSILE. 5

23) SSJ.TENSILE. 6

25) CHEVRON. 5

27) CHEVRON. 6

29) SSJ.TENSILE.7

31) SSJ.TENSILE.8

33) CHEVRON. 7

35) CHEVRON. 8

37) THERMOMETRY.1

38) THERMOMETRY.2

39) THERMOMETRY. 3

40) THERMOMETRY.4

41) HOUSING

\begin{tabular}{l} 
Material \\
\hline AL-6061 \\
SS304 \\
F82H \\
F82H \\
F82H \\
F82H \\
F82H \\
F82H \\
F82H \\
F82H \\
F82H \\
F82H \\
F82H \\
F82H \\
F82H \\
F82H \\
F82H \\
F82H \\
SiC(Irr) \\
SiC(Irr) \\
SiC(Irr) \\
SiC(Irr)
\end{tabular}

AL-6061
Therma 1

$\left(\mathrm{W} / \mathrm{m} \cdot{ }^{\circ} \mathrm{C}\right)$ 176.000

20.942

33.686

33.717

33.713

33.687

33.689

33.720

33.728

33.691

33.686

33.716

33.712

33.687

33.686

33.717

33.714

33.687

6.039

6.039

6.040

6.039

166.638
Thermal

Exp. Coeff: Emis $\left(\mu \mathrm{m} / \mathrm{m} \cdot{ }^{\circ} \mathrm{C}\right) \quad(---)$

$25.58 \quad 0.058$

$0.00 \quad 0.143$

$11.48 \quad 0.143$

$11.41 \quad 0.143$

$11.42 \quad 0.143$

$11.48 \quad 0.143$

$11.47 \quad 0.143$

$11.41 \quad 0.143$

$11.39 \quad 0.143$

$11.47 \quad 0.143$

$11.48 \quad 0.143$

$11.41 \quad 0.143$

$11.42 \quad 0.143$

$11.48 \quad 0.143$

$11.48 \quad 0.143$

$11.41 \quad 0.143$

$11.42 \quad 0.143$

$11.48 \quad 0.143$

$3.45 \quad 0.900$

$3.45 \quad 0.900$

$3.44 \quad 0.900$

$\begin{array}{ll}3.45 & 0.900\end{array}$

STORED ENERGY SUMMARY AT THE AVERAGE PART TEMPERATURE

Name

1) HOLDER

3) ROLLPIN

5) SSJ.TENSILE.1

7) SSJ.TENSILE. 2

9) CHEVRON.1

11) CHEVRON. 2

13) SSJ.TENSILE. 3

15) SSJ.TENSILE. 4

$\begin{array}{lrrrr}\text { Material } & \begin{array}{c}\text { Mass } \\ (\mathrm{g})\end{array} & \begin{array}{c}\text { Tavg } \\ \left({ }^{\circ} \mathrm{C}\right)\end{array} & \begin{array}{c}\text { Specific } \\ \mathrm{Heat} \\ \left(\mathrm{J} / \mathrm{kg}^{\circ} \mathrm{C}\right)\end{array} & \begin{array}{c}\text { Stored } \\ \text { Energy } \\ (\mathrm{J})\end{array} \\ ------ & ---- & ---- & ----- & ----- \\ \text { AL-6061 } & 1.299 & 320 . & 1038 . & 404 . \\ \text { SS304 } & 0.223 & 409 . & 396 . & 34 . \\ \text { F82H } & 0.238 & 360 . & 577 . & 47 . \\ \text { F82H } & 0.238 & 346 . & 571 . & 44 . \\ \text { F82H } & 0.115 & 347 . & 572 . & 21 . \\ \text { F82H } & 0.115 & 359 . & 577 . & 22 . \\ \text { F82H } & 0.238 & 358 . & 577 . & 46 . \\ \text { F82H } & 0.238 & 344 . & 571 . & 44 .\end{array}$


17) CHEVRON. 3

19) CHEVRON. 4

21) SSJ.TENSILE.5

23) SSJ .TENSILE.6

25) CHEVRON. 5

27) CHEVRON. 6

29) SSJ.TENSILE. 7

31) SSJ .TENSILE.8

33) CHEVRON. 7

35) CHEVRON .8

37) THERMOMETRY.1

38) THERMOMETRY.2

39) THERMOMETRY. 3

40) THERMOMETRY. 4

41) HOUSING

$\begin{array}{lrrrr}\text { F82H } & 0.115 & 341 . & 569 . & 21 . \\ \text { F82H } & 0.115 & 358 . & 576 . & 22 . \\ \text { F82H } & 0.238 & 360 . & 577 . & 47 . \\ \text { F82H } & 0.238 & 346 . & 571 . & 44 . \\ \text { F82H } & 0.115 & 348 . & 572 . & 21 . \\ \text { F82H } & 0.115 & 359 . & 577 . & 22 . \\ \text { F82H } & 0.238 & 360 . & 577 . & 47 . \\ \text { F82H } & 0.238 & 346 . & 571 . & 44 . \\ \text { F82H } & 0.115 & 347 . & 572 . & 21 . \\ \text { F82H } & 0.115 & 359 . & 577 . & 22 . \\ \text { SiC(Irr) } & 0.092 & 364 . & 1054 . & 34 . \\ \text { SiC(Irr) } & 0.092 & 364 . & 1054 . & 34 . \\ \text { SiC(Irr) } & 0.092 & 363 . & 1053 . & 33 . \\ \text { SiC(Irr) } & 0.092 & 365 . & 1054 . & 34 . \\ \text { AL-6061 } & 1.026 & 60 . & 884 . & 36 . \\ & ----10- & & & -1147 .\end{array}$

RADIAL DIMENSIONS AND GAP SUMMARY FOR THE CORE-HOUSING GAP

CONTACT SUMMARY FOR CONTACT ID 83: Frictionless - HOLDER TO HOUSING

Contact surface material:

Target surface material:

Interstitial gas:

Effective surface roughness:

Effective asperity slope:

Effective microhardness:

$$
\begin{aligned}
& \text { AL-6061 } \\
& \text { AL-6061 } \\
& \text { He1 1 um } \\
& 2.263 \mu \mathrm{m} \\
& 0.214 \mathrm{rad} \\
& 1.220 \mathrm{GPa}
\end{aligned}
$$

\begin{tabular}{|c|c|c|c|}
\hline & Average & Minimum & Maximum \\
\hline direct results & & & \\
\hline $\begin{array}{l}\text { Contact status } \\
\text { contact temperature }\left({ }^{\circ} \mathrm{C}\right)\end{array}$ & $\begin{array}{r}1.000 \\
318.390\end{array}$ & $\begin{array}{r}1.000 \\
314.987\end{array}$ & $\begin{array}{r}1.000 \\
320.231\end{array}$ \\
\hline Target temperature $\left({ }^{\circ} \mathrm{C}\right)$ & $\begin{aligned} 310.590 \\
60.549\end{aligned}$ & $\begin{array}{r}314.901 \\
60.056\end{array}$ & $\begin{array}{r}320.231 \\
60.660\end{array}$ \\
\hline Geometric gas gap $(\mu \mathrm{m})$ & 184.970 & 184.583 & 185.210 \\
\hline Contact pressure (MPa) & 0.000 & 0.000 & 0.000 \\
\hline Gap conduction heat f1ux $\left(\mathrm{kW} / \mathrm{m}^{2}\right)$ & 338.954 & 332.675 & 342.249 \\
\hline Radiation heat flux $\left(\mathrm{kW} / \mathrm{m}^{2}\right)$ & 0.000 & 0.000 & 0.000 \\
\hline Contact conduction heat flux $\left(\mathrm{kW} / \mathrm{m}^{2}\right)$ & 0.000 & 0.000 & 0.00 \\
\hline Total heat flux $\left(\mathrm{kW} / \mathrm{m}^{2}\right)$ & 338.954 & 332.67 & 342.24 \\
\hline Therma 1 contact conductance $\left(\mathrm{W} / \mathrm{m}^{2} \cdot \mathrm{C}\right)$ & 1314.571 & 1306.42 & $1320.1 \mathrm{~s}$ \\
\hline Effective gas gap ( $\mu \mathrm{m})$ & 154 & 154 & 155. \\
\hline Contact therma1 jump distance $(\mu \mathrm{m})$ & & 1 & $4 / 5$ \\
\hline Target thermal jump distance $(\mu \mathrm{m})$ & 1.290 & 1.284 & 7 \\
\hline Effective contact pressure (MPa) & & 0.000 & \\
\hline Pressure index & 13.534 & 13.534 & 13.5 \\
\hline Gas thermal conductivity $\left(\mathrm{W} / \mathrm{m} \cdot{ }^{\circ} \mathrm{C}\right)$ & & 0.2 & \\
\hline Solid spot conductance $\left(\mathrm{W} / \mathrm{m}^{2} \cdot \mathrm{C}\right)$ & 0.000 & 0.000 & 0.000 \\
\hline Gas gap conduc & 1313.812 & 1305.817 & 1319.370 \\
\hline
\end{tabular}

Contact status codes:

$0=$ open/no heat transfer, $1=$ near-field contact $2=$ closed and sliding, $\quad 3=$ closed and sticking 
$9.00 \mathrm{~mm}$ holder diameter (tensile design)

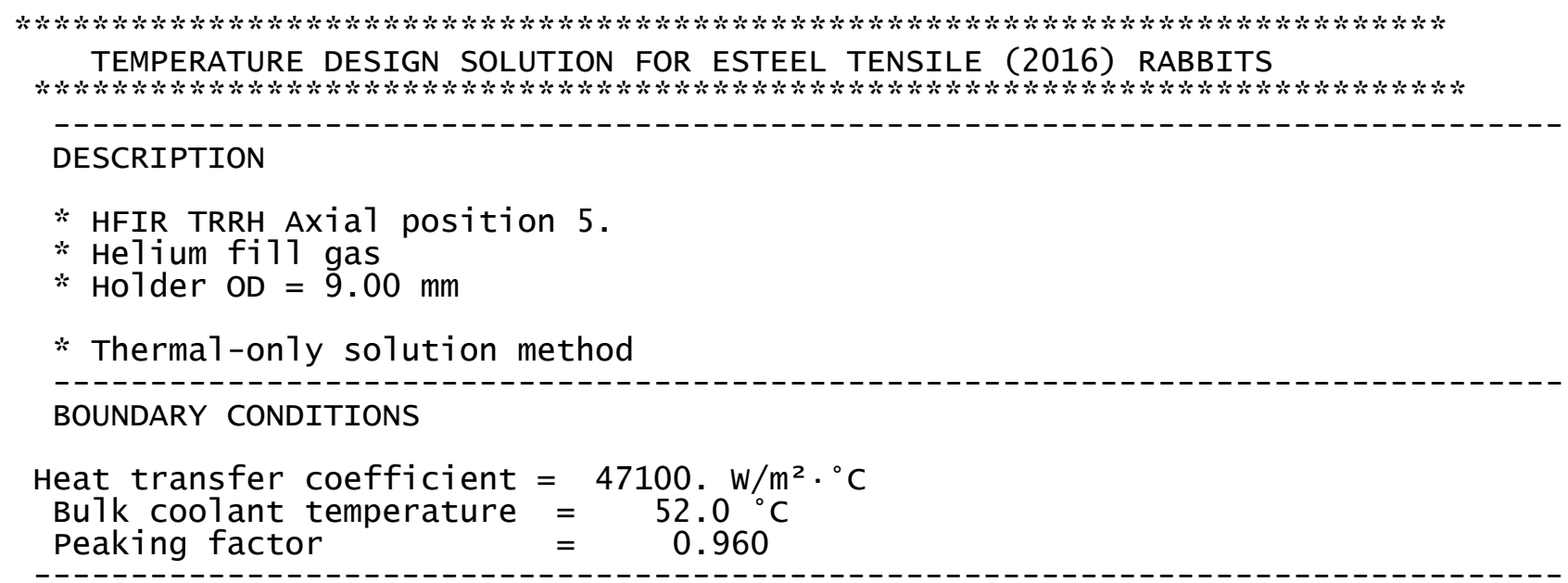

HEAT GENERATION

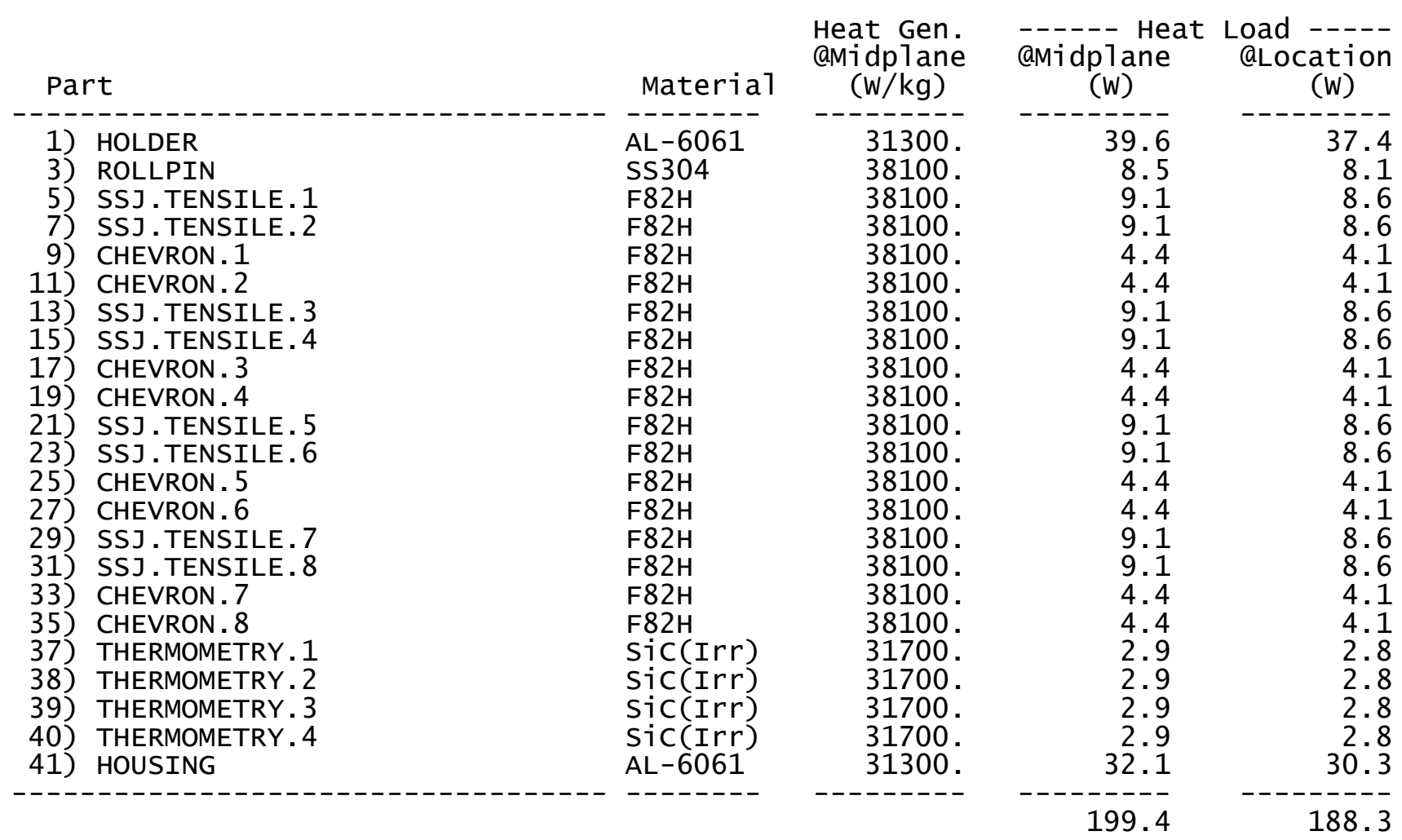

CAPSULE TEMPERATURE SUMMARY

\begin{tabular}{|c|c|c|c|c|c|c|c|}
\hline Name & & Material & Tavg & Tmin & Tmax & T. 025 & T. 975 \\
\hline $\begin{array}{r}1) \\
3) \\
5) \\
7) \\
9) \\
11) \\
13)\end{array}$ & $\begin{array}{l}\text { HOLDER } \\
\text { ROLLPIN } \\
\text { SSJ.TENSILE. } 1 \\
\text { SSJ.TENSILE. } 2 \\
\text { CHEVRON. } 1 \\
\text { CHEVRON. } 2 \\
\text { SSJ.TENSILE. } 3\end{array}$ & $\begin{array}{l}\text { AL-6061 } \\
\text { SS304 } \\
\text { F82H } \\
\text { F82H } \\
\text { F82H } \\
\text { F82H } \\
\text { F82H }\end{array}$ & $\begin{array}{l}348 . \\
437 . \\
388 . \\
374 . \\
375 . \\
386 . \\
388 .\end{array}$ & $\begin{array}{l}344 . \\
431 . \\
368 . \\
363 . \\
367 . \\
374 . \\
368 .\end{array}$ & $\begin{array}{l}352 . \\
447 . \\
399 . \\
382 . \\
383 . \\
394 . \\
399 .\end{array}$ & $\begin{array}{l}345 . \\
432 . \\
373 . \\
366 . \\
371 . \\
380 . \\
373 .\end{array}$ & $\begin{array}{l}351 . \\
444 . \\
397 . \\
380 . \\
381 . \\
392 . \\
397 .\end{array}$ \\
\hline
\end{tabular}


15) SSJ .TENSILE . 4

17) CHEVRON. 3

19) CHEVRON. 4

21) SSJ.TENSILE. 5

23) SSJ .TENSILE. 6

25) CHEVRON. 5

27) CHEVRON. 6

29) SSJ.TENSILE.7

31) SSJ .TENSILE. 8

33) CHEVRON.7

35) CHEVRON.8

37) THERMOMETRY.1

38) THERMOMETRY. 2

39) THERMOMETRY .3

40) THERMOMETRY.4

41) HOUSING
$\mathrm{F} 82 \mathrm{H}$

$\mathrm{F} 82 \mathrm{H}$

$\mathrm{F} 82 \mathrm{H}$

$\mathrm{F} 82 \mathrm{H}$

$\mathrm{F} 82 \mathrm{H}$

$\mathrm{F} 82 \mathrm{H}$

$\mathrm{F} 82 \mathrm{H}$

$\mathrm{F} 82 \mathrm{H}$

$\mathrm{F} 82 \mathrm{H}$

$\mathrm{F} 82 \mathrm{H}$

$\mathrm{F} 82 \mathrm{H}$

$\operatorname{SiC}(\operatorname{Ir} r)$

Sic(Irr)

SiC(Irr)

$\operatorname{sic}(\operatorname{Ir} r)$
374. 363. 382.

375. 357. 383.

387. 373. 395.

388 . 368. 399.

374. 364. 383.

375.367 .383$.

387.373 .394$.

388. 368. 399 .

374. 363. 382 .

375. 366. 383.

386. 371 . 394

392. 356.417

392. 356.417.

392. 357.420.

393. 357.421.
366. 380 .

369 . 381.

380. 393.

373. 397.

366 . $\quad 380$.

371. 381 .

380.392.

373. 397.

$366 . \quad 379$.

371. $\quad 381$.

380.392.

368. 408.

368. 408 .

368. 409.

369. 409.

PROPERTY SUMMARY AT THE AVERAGE PART TEMPERATURE

Name

1) HOLDER

3) ROLLPIN

5) SSJ.TENSILE.1

7) SSJ.TENSILE.2

9) CHEVRON.1

11) CHEVRON. 2

13) SSJ.TENSILE. 3

15) SSJ.TENSILE. 4

17) CHEVRON. 3

19) CHEVRON. 4

21) SSJ.TENSILE. 5

23) SSJ.TENSILE. 6

25) CHEVRON. 5

27) CHEVRON. 6

29) SSJ.TENSILE.7

31) SSJ.TENSILE.8

33) CHEVRON.7

35) CHEVRON. 8

37) THERMOMETRY.1

38) THERMOMETRY.2

39) THERMOMETRY. 3

40) THERMOMETRY.4

41) HOUSING

Material
---6061
AL-601
SS304
F82H
F82H
F82H
F82H
F82H
F82H
F82H
F82H
F82H
F82H
F82H
F82H
F82H
F82H
F82H
F82H
SiC(Irr)
SiC(Irr)
SiC(Irr)
SiC(Irr)

AL-6061

\section{Therma1 \\ cond.}

$\left(\mathrm{W} / \mathrm{m} \cdot{ }^{\circ} \mathrm{C}\right)$

176.000

21.324

33.625

33.655

33.652

33.628

33.625

33.655

33.653

33.626

33.624

33.655

33.652

33.627

33.625

33.655

33.652

33.628

6.028

6.028

6.028

6.028

166.633
Thermal

Exp. Coeff: Emis $\left(\mu \mathrm{m} / \mathrm{m} \cdot{ }^{\circ} \mathrm{C}\right)(---)$ $25.85 \quad 0.060$ $0.00 \quad 0.143$

$11.61 \quad 0.143$

$11.55 \quad 0.143$

$11.56 \quad 0.143$

$11.61 \quad 0.143$

$11.61 \quad 0.143$

$11.55 \quad 0.143$

$11.55 \quad 0.143$

$11.61 \quad 0.143$

$11.62 \quad 0.143$

$11.55 \quad 0.143$

$11.56 \quad 0.143$

$11.61 \quad 0.143$

$11.61 \quad 0.143$

$11.55 \quad 0.143$

$11.55 \quad 0.143$

$11.61 \quad 0.143$

$3.52 \quad 0.900$

$3.52 \quad 0.900$

$3.53 \quad 0.900$

$3.53 \quad 0.900$

STORED ENERGY SUMMARY AT THE AVERAGE PART TEMPERATURE

Name

1) HOLDER

3) ROLLPIN

5) SSJ.TENSILE.1

7) SSJ.TENSILE. 2

9) CHEVRON.1

11) CHEVRON. 2

13) SSJ.TENSILE. 3

15) SSJ.TENSILE.4

\begin{tabular}{|c|c|c|c|c|}
\hline aterial & $\begin{array}{r}\text { Mass } \\
(\mathrm{g})\end{array}$ & $\begin{array}{l}\text { Tavg } \\
\left({ }^{\circ} \mathrm{C}\right)\end{array}$ & $\begin{array}{l}\text { Specific } \\
\text { Heat } \\
\left(\mathrm{J} / \mathrm{kg}^{\circ} \mathrm{C}\right)\end{array}$ & $\begin{array}{c}\text { Stored } \\
\text { Energy } \\
(\mathrm{J})\end{array}$ \\
\hline $\begin{array}{l}\text { SS3O4 } \\
\text { F82H } \\
\text { F82H } \\
\text { F82H } \\
\text { F82H } \\
\text { F82H } \\
\text { F82H }\end{array}$ & $\begin{array}{l}1.265 \\
0.224 \\
0.238 \\
0.238 \\
0.115 \\
0.115 \\
0.238 \\
0.238\end{array}$ & $\begin{array}{l}4 \\
3 \\
3 \\
3 \\
3 \\
3 \\
3 \\
3\end{array}$ & $\begin{array}{r}1051 . \\
399 . \\
589 . \\
583 . \\
584 . \\
588 . \\
589 . \\
583 .\end{array}$ & $\begin{array}{r}437 \\
37 \\
52 \\
49\end{array}$ \\
\hline
\end{tabular}


17) CHEVRON. 3

19) CHEVRON. 4

21) SSJ.TENSILE.5

23) SSJ .TENSILE.6

25) CHEVRON. 5

27) CHEVRON. 6

29) SSJ.TENSILE.7

31) SSJ.TENSILE.8

33) CHEVRON.7

35) CHEVRON. 8

37) THERMOMETRY.1

38) THERMOMETRY.2

39) THERMOMETRY. 3

40) THERMOMETRY. 4

41) HOUSING

$\begin{array}{lrrrr}\text { F82H } & 0.115 & 375 . & 584 . & 24 . \\ \text { F82H } & 0.115 & 387 . & 589 . & 25 . \\ \text { F82H } & 0.238 & 388 . & 589 . & 52 . \\ \text { F82H } & 0.238 & 374 . & 583 . & 49 . \\ \text { F82H } & 0.115 & 375 . & 584 . & 24 . \\ \text { F82H } & 0.115 & 387 . & 588 . & 25 . \\ \text { F82H } & 0.238 & 388 . & 589 . & 51 . \\ \text { F82H } & 0.238 & 374 . & 583 . & 49 . \\ \text { F82H } & 0.115 & 375 . & 584 . & 24 . \\ \text { F82H } & 0.115 & 386 . & 588 . & 25 . \\ \text { SiC(Irr) } & 0.092 & 392 . & 1069 . & 37 . \\ \text { SiC(Irr) } & 0.092 & 392 . & 1069 . & 37 . \\ \text { SiC(Irr) } & 0.092 & 392 . & 1069 . & 37 . \\ \text { SiC(Irr) } & 0.092 & 393 . & 1069 . & 37 . \\ \text { AL-6061 } & 1.026 & 60 . & 884 . & 36 . \\ & ---1.0- & & & --1253 .\end{array}$

RADIAL DIMENSIONS AND GAP SUMMARY FOR THE CORE-HOUSING GAP

CONTACT SUMMARY FOR CONTACT ID 83: Frictionless - HOLDER TO HOUSING

Contact surface material:

Target surface material:

Interstitial gas:

Effective surface roughness:

Effective asperity slope:

Effective microhardness:

$$
\begin{aligned}
& \text { AL-6061 } \\
& \text { AL-6061 } \\
& \text { He1 1 um } \\
& 2.263 \mu \mathrm{m} \\
& 0.214 \mathrm{rad} \\
& 1.220 \mathrm{GPa}
\end{aligned}
$$

\begin{tabular}{|c|c|c|c|}
\hline & Average & Minimum & Maximum \\
\hline direct results & & & \\
\hline $\begin{array}{l}\text { Contact status } \\
\text { Contact temperature }\left({ }^{\circ} \mathrm{C}\right)\end{array}$ & $\begin{array}{r}1.000 \\
347.127\end{array}$ & $\begin{array}{r}1.000 \\
343.657\end{array}$ & $\begin{array}{r}1.000 \\
348.796\end{array}$ \\
\hline Target temperature $\left({ }^{\circ} \mathrm{C}\right)$ & $\begin{array}{r}341.521 \\
60.506\end{array}$ & $\begin{array}{r}345.031 \\
60.021\end{array}$ & $\begin{array}{r}340.790 \\
60.617\end{array}$ \\
\hline Geometric gas gap $(\mu \mathrm{m})$ & 209.964 & 209.691 & 210.178 \\
\hline Contact pressure (MPa) & 0.000 & 0.000 & 0.000 \\
\hline Gap conduction heat f1ux $\left(\mathrm{kW} / \mathrm{m}^{2}\right)$ & 338.734 & 332.727 & 341.79 \\
\hline Radiation heat flux $\left(\mathrm{kW} / \mathrm{m}^{2}\right)$ & 0.000 & 0.000 & 0.000 \\
\hline Contact conduction heat flux $\left(\mathrm{kW} / \mathrm{m}^{2}\right)$ & 0.000 & 0.000 & 0.00 \\
\hline Total heat flux $\left(\mathrm{kW} / \mathrm{m}^{2}\right)$ & 338.734 & 332.72 & 341.79 \\
\hline Therma 1 contact conductance $\left(\mathrm{W} / \mathrm{m}^{2} \cdot \mathrm{C}\right)$ & 1181.80 & 1174.51 & 1186.19 \\
\hline 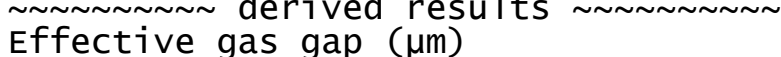 & 175 & 175. & 176 \\
\hline Contact therma1 jump distance $(\mu \mathrm{m})$ & & & 547 \\
\hline Target thermal jump distance $(\mu \mathrm{m})$ & 1.339 & 1.33 & 1.342 \\
\hline Effective contact pressure (MPa) & & & 000 \\
\hline Pressure index & 13.534 & 13.53 & 13.5 \\
\hline Gas thermal conductivity $\left(\mathrm{W} / \mathrm{m} \cdot{ }^{\circ} \mathrm{C}\right)$ & & 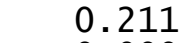 & 0 . \\
\hline Solid spot conductance $\left(\mathrm{W} / \mathrm{m}^{2} \cdot \mathrm{C}\right)$ & 0. & 0. & 0.000 \\
\hline Gas gap & 1181.32 & 1173.988 & 1185.726 \\
\hline
\end{tabular}

Contact status codes:

$0=$ open/no heat transfer, $1=$ near-field contact $2=$ closed and sliding, $\quad 3=$ closed and sticking 
$9.44 \mathrm{~mm}$ holder diameter (bend bar design)

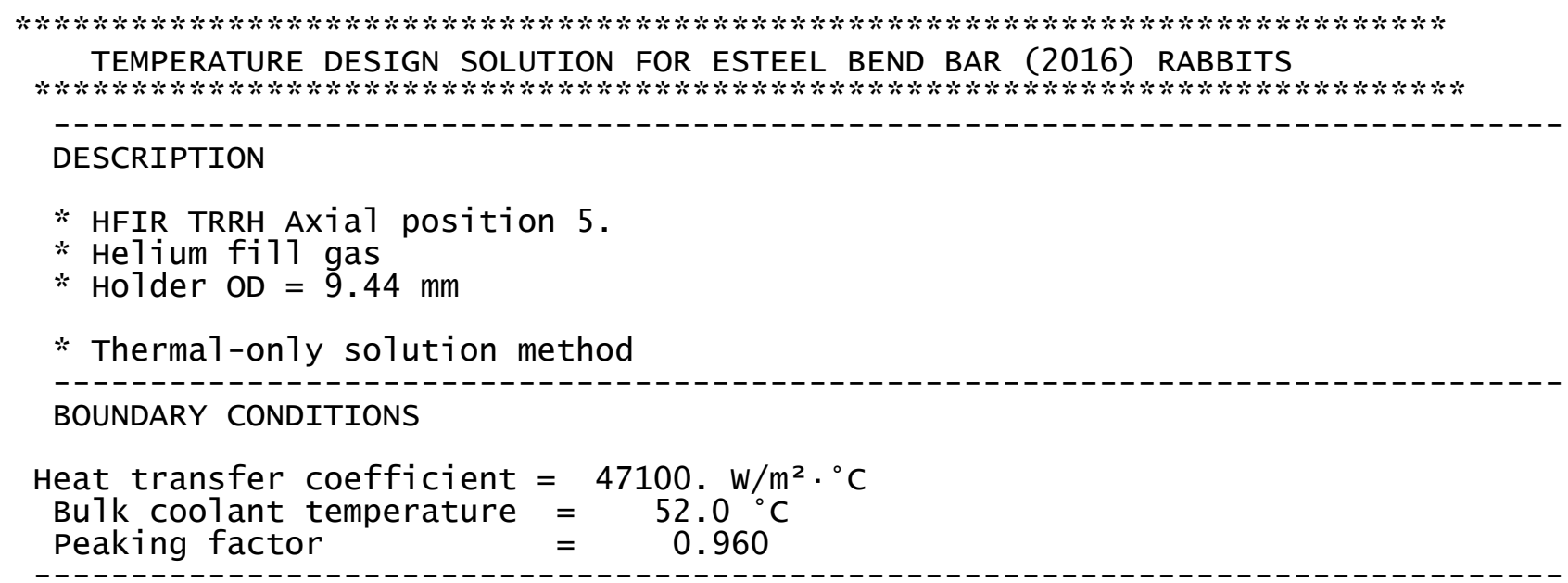

HEAT GENERATION

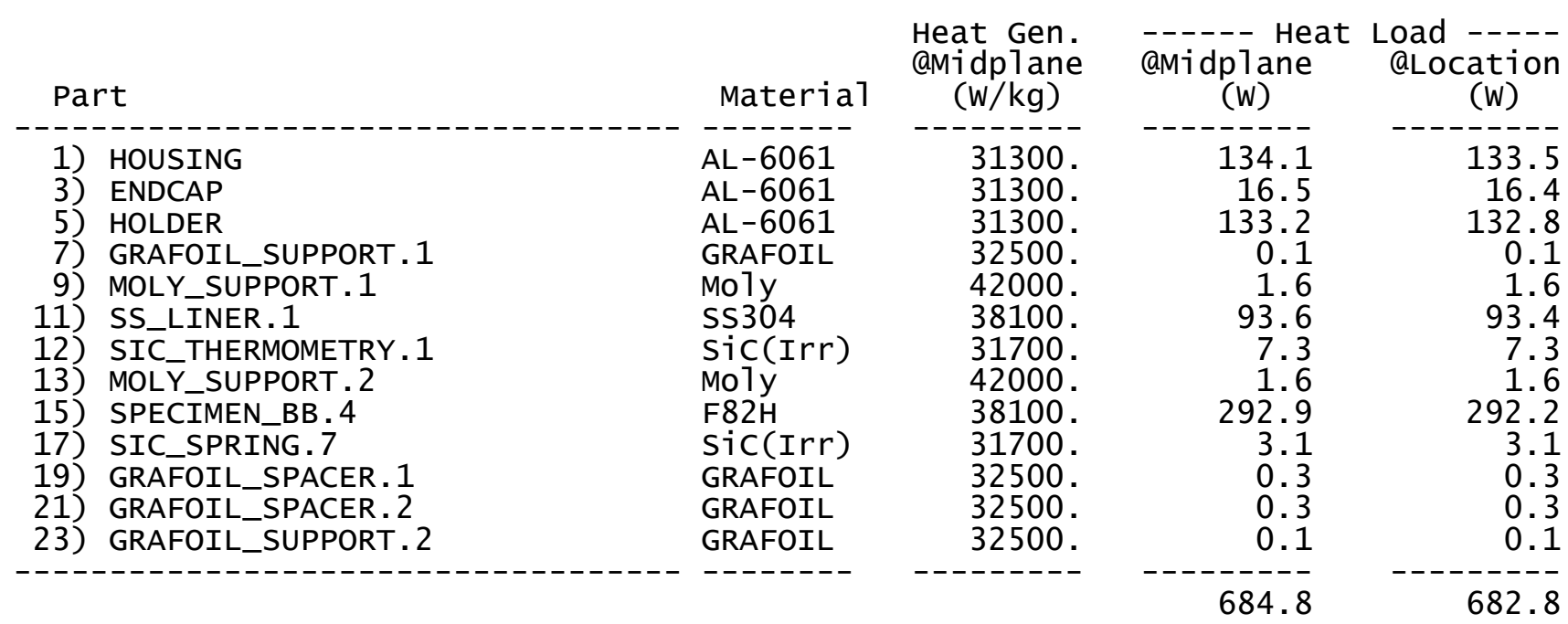

CAPSULE TEMPERATURE SUMMARY

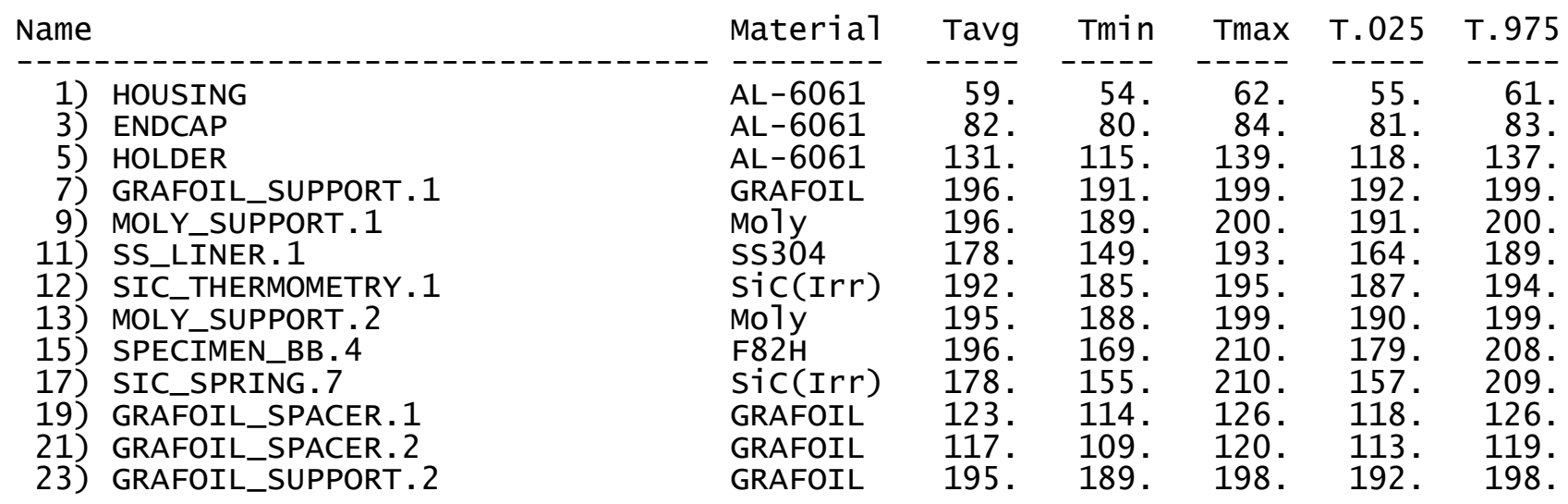


Name

1) HOUSING
3) ENDCAP
5) HOLDER
7) GRAFOIL_SUPPORT . 1
9) MOLY_SUPPORT. 1
11) SS_LINER.1
12) SIC_THERMOMETRY . 1
13) MOLY_SUPPORT . 2
15) SPECIMEN_BB . 4
17) SIC_SPRING . 7
19) GRAFOIL_SPACER. 1
21) GRAFOIL_SPACER . 2
23) GRAFOIL_SUPPORT . 2

\begin{tabular}{|c|c|c|c|}
\hline aterial & $\begin{array}{l}\text { Therma1 } \\
\text { cond. } \\
\left(\mathrm{W} / \mathrm{m} \cdot{ }^{\circ} \mathrm{C}\right)\end{array}$ & $\begin{array}{l}\text { Therma1 } \\
\text { Exp. } \\
\text { Coeff. } \\
\left(\mu \mathrm{m} / \mathrm{m} \cdot{ }^{\circ} \mathrm{C}\right)\end{array}$ & $\begin{array}{l}\text { Emis } \\
(---)\end{array}$ \\
\hline $\begin{array}{l}061 \\
061 \\
0 I L \\
4 \\
\text { Irr) } \\
\text { Irr) } \\
\text { OIL }\end{array}$ & $\begin{array}{r}166.589 \\
169.242 \\
174.116 \\
38.000 \\
131.252 \\
17.714 \\
6.106 \\
131.287 \\
33.207 \\
6.111 \\
38.000 \\
38.000 \\
38.000\end{array}$ & $\begin{array}{r}24.21 \\
0.00 \\
24.23 \\
1.00 \\
0.00 \\
0.00 \\
2.90 \\
0.00 \\
11.01 \\
2.85 \\
1.00 \\
1.00 \\
1.00\end{array}$ & $\begin{array}{l}0.050 \\
0.050 \\
0.050 \\
0.500 \\
0.047 \\
0.143 \\
0.900 \\
0.047 \\
0.143 \\
0.900 \\
0.500 \\
0.500 \\
0.500\end{array}$ \\
\hline
\end{tabular}

STORED ENERGY SUMMARY AT THE AVERAGE PART TEMPERATURE

\begin{tabular}{|c|c|c|c|c|c|c|}
\hline Name & & Material & $\begin{array}{r}\text { Mass } \\
(\mathrm{g})\end{array}$ & $\begin{array}{l}\text { Tavg } \\
\left({ }^{\circ} \mathrm{C}\right)\end{array}$ & $\begin{array}{c}\text { Specific } \\
\text { Heat } \\
\left(\mathrm{J} / \mathrm{kg}^{\circ} \mathrm{C}\right)\end{array}$ & $\begin{array}{l}\text { Stored } \\
\text { Energy } \\
\text { (J) }\end{array}$ \\
\hline $\begin{array}{r}1) \\
3) \\
5) \\
7) \\
9) \\
11) \\
12) \\
13) \\
15) \\
17) \\
19) \\
21) \\
23)\end{array}$ & $\begin{array}{l}\text { HOUSING } \\
\text { ENDCAP } \\
\text { HOLDER } \\
\text { GRAFOIL_SUPPORT.1 } \\
\text { MOLY_SUPPORT. } 1 \\
\text { SS_LINER.1 } \\
\text { SIC_THERMOMETRY.1 } \\
\text { MOLY_SUPPORT. } 2 \\
\text { SPECIMEN_BB. } 4 \\
\text { SIC_SPRING.7 } \\
\text { GRAFOIL_SPACER.1 } \\
\text { GRAFOIL_SPACER. } 2 \\
\text { GRAFOIL_SUPPORT. } 2\end{array}$ & $\begin{array}{l}\text { AL-6061 } \\
\text { AL-6061 } \\
\text { AL-6061 } \\
\text { GRAFOIL } \\
\text { Mo7y } \\
\text { SS304 } \\
\text { SiC(Irr) } \\
\text { Moly } \\
\text { F82H } \\
\text { SiC(Irr) } \\
\text { GRAFOIL } \\
\text { GRAFOIL } \\
\text { GRAFOIL }\end{array}$ & $\begin{array}{l}4.286 \\
0.527 \\
4.255 \\
0.004 \\
0.039 \\
2.457 \\
0.231 \\
0.039 \\
7.687 \\
0.097 \\
0.009 \\
0.009 \\
0.004\end{array}$ & $\begin{array}{l}59 . \\
82 . \\
131 . \\
196 . \\
196 . \\
178 . \\
192 . \\
195 . \\
196 . \\
178 . \\
123 . \\
117 . \\
195 .\end{array}$ & $\begin{array}{l}884 . \\
902 . \\
937 . \\
700 . \\
262 . \\
381 . \\
934 . \\
262 . \\
514 . \\
918 . \\
700 . \\
700 . \\
700 .\end{array}$ & $\begin{array}{r}149 . \\
29 . \\
441 . \\
0 . \\
2 . \\
148 . \\
37 . \\
2 . \\
696 . \\
14 . \\
1 . \\
1 . \\
0 .\end{array}$ \\
\hline & & & 19.643 & & & 1520. \\
\hline
\end{tabular}

RADIAL DIMENSIONS AND GAP SUMMARY FOR THE CORE-HOUSING GAP

CONTACT SUMMARY FOR CONTACT ID 25: Frictionless - HOLDER TO HOUSING

Contact surface material:

Target surface material:

Interstitial gas:

Effective surface roughness:

Effective asperity slope:

Effective microhardness:
AL-6061

AL-6061

Helium

$2.263 \mu \mathrm{m}$

$0.214 \mathrm{rad}$

1.220 GPa

$\begin{array}{lrrr} & \text { Average } & \text { Minimum } & \text { Maximum } \\ \text { c n direct results } & & & \\ \text { Contact status } & 1.000 & 1.000 & 1.000 \\ \text { Contact temperature }\left({ }^{\circ} \mathrm{C}\right) & 61.983 & 114.642 & 135.260 \\ \text { Target temperature }\left({ }^{\circ} \mathrm{C}\right) & 38.430 & 57.355 & 62.170 \\ \text { Geometric gas gap }(\mu \mathrm{m}) & 0.000 & 17.100 & 38.473 \\ \text { Contact pressure }(\mathrm{MPa}) & 364.449 & 288.000 & 0.000 \\ \text { Gap conduction heat flux }\left(\mathrm{kW} / \mathrm{m}^{2}\right) & 0.000 & 0.000 & 934.828 \\ \text { Radiation heat flux }\left(\mathrm{kW} / \mathrm{m}^{2}\right) & & & 0.000\end{array}$




$\begin{array}{lrrr}\text { Contact conduction heat flux }\left(\mathrm{kW} / \mathrm{m}^{2}\right) & 0.000 & 0.000 & 0.000 \\ \text { Total heat flux }\left(\mathrm{kW} / \mathrm{m}^{2}\right) & 364.449 & 288.881 & 934.828 \\ \text { Therma1 contact conductance }\left(\mathrm{W} / \mathrm{m}^{2} \cdot \mathrm{C}\right) & 5371.732 & 5084.671 & 14705.808 \\ \text { ～ derived results } & & & \\ \text { Effective gas gap }(\mu \mathrm{m}) & 30.672 & 9.835 & 31.967 \\ \text { Contact therma1 jump distance }(\mu \mathrm{m}) & 1.016 & 0.978 & 1.032 \\ \text { Target therma1 jump distance }(\mu \mathrm{m}) & 0.976 & 0.944 & 0.988 \\ \text { Effective contact pressure }(\mathrm{MPa}) & 0.000 & 0.000 & 0.000 \\ \text { Pressure index } & 13.534 & 13.534 & 13.534 \\ \text { Gas thermal conductivity }\left(\mathrm{W} / \mathrm{m} \cdot{ }^{\circ} \mathrm{C}\right) & 0.175 & 0.172 & 0.177 \\ \text { Solid spot conductance }\left(\mathrm{W} / \mathrm{m}^{2} \cdot \mathrm{C}\right) & 0.000 & 0.000 & 0.000 \\ \text { Gas gap conductance }\left(\mathrm{W} / \mathrm{m}^{2} \cdot \mathrm{C}\right) & 5376.230 & 5083.350 & 14693.840\end{array}$

Contact status codes:

$0=$ open/no heat transfer, $1=$ near-field contact

$2=$ closed and sliding, $\quad 3=c l o s e d$ and sticking 
$9.40 \mathrm{~mm}$ holder diameter (bend bar design)

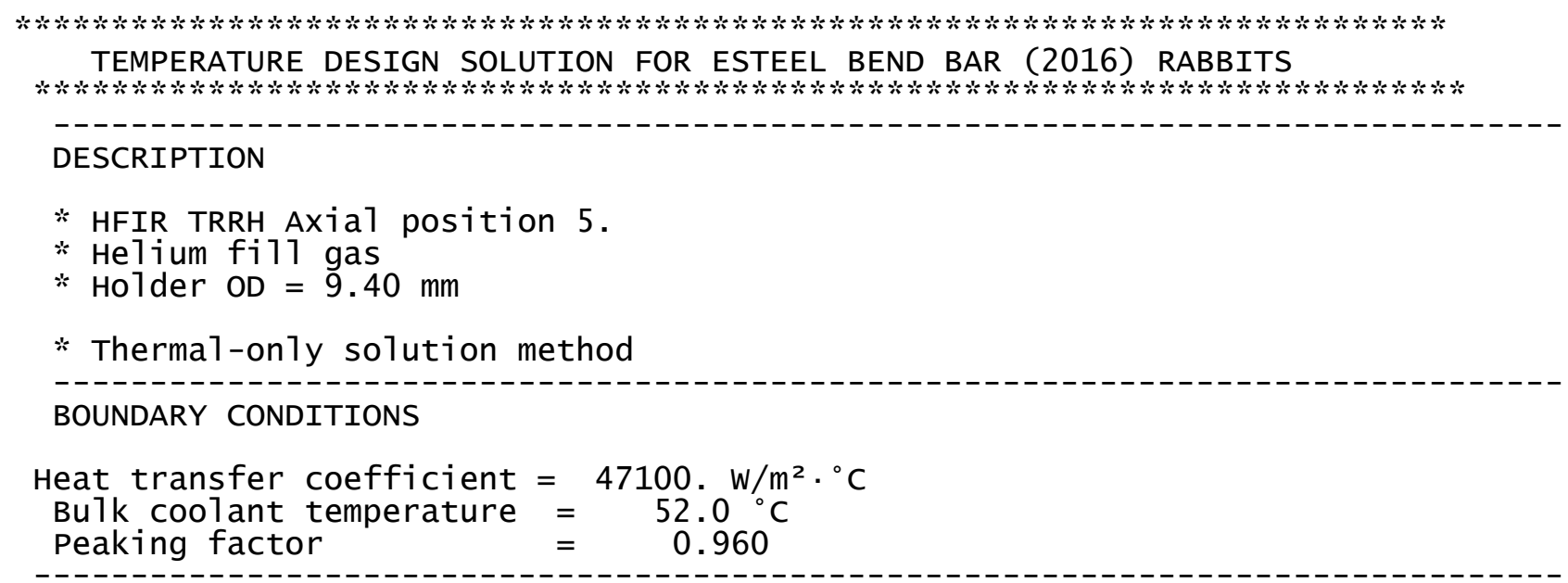

HEAT GENERATION

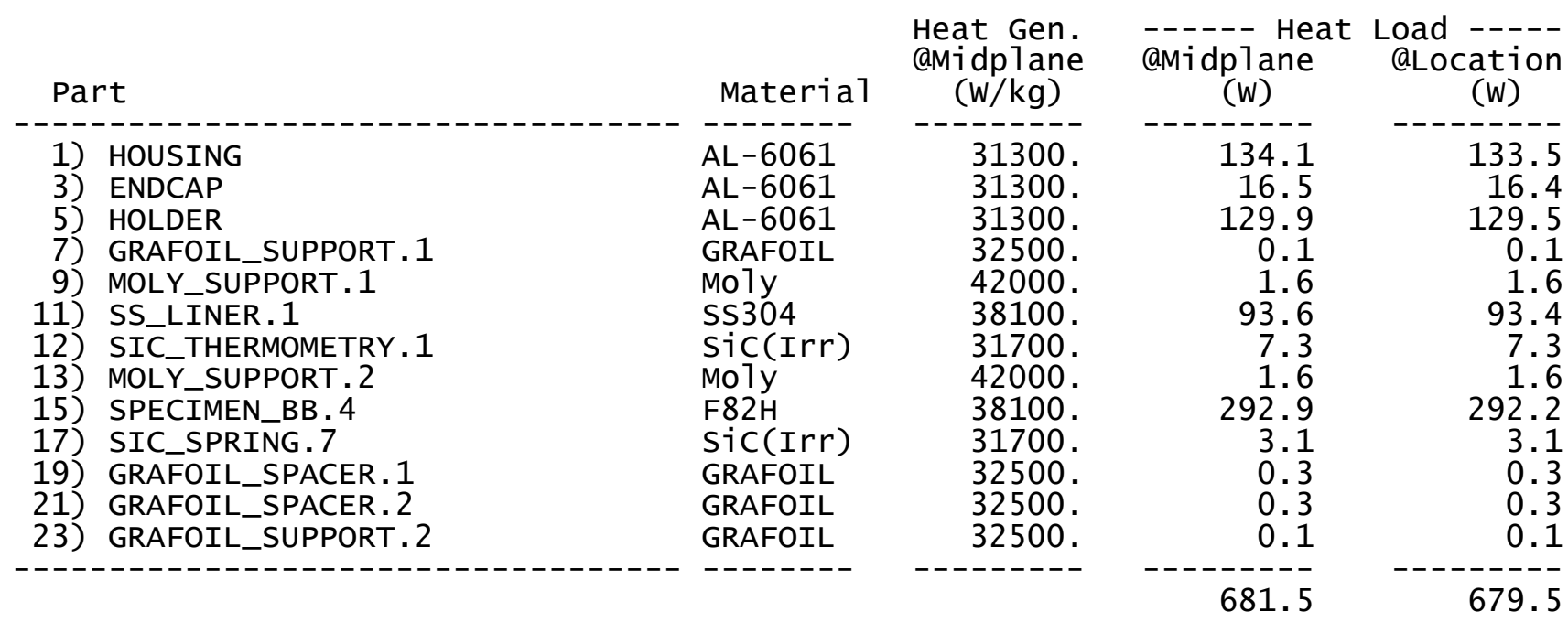

CAPSULE TEMPERATURE SUMMARY

\begin{tabular}{|c|c|c|c|c|c|c|c|}
\hline Name & & Material & Tavg & Tmin & Tmax & T. 025 & T. 975 \\
\hline $\begin{array}{r}1) \\
3) \\
5) \\
7) \\
9) \\
11) \\
12) \\
13) \\
15) \\
17) \\
19) \\
21) \\
23)\end{array}$ & $\begin{array}{l}\text { HOUSING } \\
\text { ENDCAP } \\
\text { HOLDER } \\
\text { GRAFOIL_SUPPORT. } 1 \\
\text { MOLY_SUPPORT. } 1 \\
\text { SS_LINER.1 } \\
\text { SIC_THERMOMETRY. } 1 \\
\text { MOLY_SUPPORT. } 2 \\
\text { SPECIMEN_BB. } 4 \\
\text { SIC_SPRING. } 7 \\
\text { GRAFOIL_SPACER.1 } \\
\text { GRAFOIL_SPACER.2 } \\
\text { GRAFOIL_SUPPORT. } 2\end{array}$ & $\begin{array}{l}\text { AL-6061 } \\
\text { AL-6061 } \\
\text { AL-6061 } \\
\text { GRAFOIL } \\
\text { Mo1y } \\
\text { SS304 } \\
\text { SiC(Irr) } \\
\text { Moly } \\
\text { F82H } \\
\text { SiC(Irr) } \\
\text { GRAFOIL } \\
\text { GRAFOIL } \\
\text { GRAFOIL }\end{array}$ & $\begin{array}{l}59 . \\
82 . \\
172 . \\
235 . \\
235 . \\
218 . \\
232 . \\
233 . \\
236 . \\
218 . \\
163 . \\
155 . \\
234 .\end{array}$ & $\begin{array}{l}54 . \\
80 . \\
153 . \\
230 . \\
228 . \\
189 . \\
223 . \\
226 . \\
207 . \\
196 . \\
148 . \\
143 . \\
227 .\end{array}$ & $\begin{array}{l}62 . \\
84 . \\
181 . \\
239 . \\
239 . \\
234 . \\
236 . \\
238 . \\
251 . \\
249 . \\
166 . \\
158 . \\
237 .\end{array}$ & $\begin{array}{l}55 . \\
81 . \\
156 . \\
231 . \\
230 . \\
204 . \\
225 . \\
228 . \\
218 . \\
198 . \\
155 . \\
149 . \\
231 .\end{array}$ & $\begin{array}{r}61 . \\
83 . \\
180 . \\
238 . \\
239 . \\
231 . \\
235 . \\
237 . \\
248 . \\
249 . \\
166 .\end{array}$ \\
\hline
\end{tabular}


Name

1) HOUSING
3) ENDCAP
5) HOLDER
7) GRAFOIL_SUPPORT . 1
9) MOLY_SUPPORT. 1
11) SS_LINER.1
12) SIC_THERMOMETRY . 1
13) MOLY_SUPPORT. 2
15) SPECIMEN_BB . 4
17) SIC_SPRING.7
19) GRAFOIL_SPACER. 1
21) GRAFOIL_SPACER. 2
23) GRAFOIL_SUPPORT. 2

\begin{tabular}{|c|c|c|c|}
\hline aterial & $\begin{array}{l}\text { Therma1 } \\
\text { cond. } \\
\left(\mathrm{W} / \mathrm{m} \cdot{ }^{\circ} \mathrm{C}\right)\end{array}$ & $\begin{array}{l}\text { Therma1 } \\
\text { Exp. } \\
\text { Coeff. } \\
\left(\mu \mathrm{m} / \mathrm{m} \cdot{ }^{\circ} \mathrm{C}\right)\end{array}$ & $\begin{array}{l}\text { Emis } \\
(---)\end{array}$ \\
\hline $\begin{array}{l}061 \\
061 \\
0 I L \\
4 \\
\text { Irr) } \\
\text { Irr) } \\
\text { OIL }\end{array}$ & $\begin{array}{r}166.586 \\
169.244 \\
176.000 \\
38.000 \\
129.681 \\
18.281 \\
6.090 \\
129.744 \\
33.423 \\
6.095 \\
38.000 \\
38.000 \\
38.000\end{array}$ & $\begin{array}{r}24.21 \\
0.00 \\
24.49 \\
1.00 \\
0.00 \\
0.00 \\
3.04 \\
0.00 \\
11.08 \\
3.00 \\
1.00 \\
1.00 \\
1.00\end{array}$ & $\begin{array}{l}0.050 \\
0.050 \\
0.051 \\
0.500 \\
0.051 \\
0.143 \\
0.900 \\
0.051 \\
0.143 \\
0.900 \\
0.500 \\
0.500 \\
0.500\end{array}$ \\
\hline
\end{tabular}

STORED ENERGY SUMMARY AT THE AVERAGE PART TEMPERATURE

\begin{tabular}{|c|c|c|c|c|c|c|}
\hline Name & & Material & $\begin{array}{r}\text { Mass } \\
(\mathrm{g})\end{array}$ & $\begin{array}{l}\text { Tavg } \\
\left({ }^{\circ} \mathrm{C}\right)\end{array}$ & $\begin{array}{c}\text { Specific } \\
\text { Heat } \\
\left(\mathrm{J} / \mathrm{kg}^{\circ} \mathrm{C}\right)\end{array}$ & $\begin{array}{l}\text { Stored } \\
\text { Energy } \\
(\mathrm{J})\end{array}$ \\
\hline $\begin{array}{r}\text { 1) } \\
3) \\
5) \\
7) \\
9) \\
11) \\
12) \\
13) \\
15) \\
17) \\
19) \\
21) \\
23)\end{array}$ & $\begin{array}{l}\text { HOUSING } \\
\text { ENDCAP } \\
\text { HOLDER } \\
\text { GRAFOIL_SUPPORT.1 } \\
\text { MOLY_SUPPORT. } 1 \\
\text { SS_LINER. } 1 \\
\text { SIC_THERMOMETRY.1 } \\
\text { MOLY_SUPPORT. } 2 \\
\text { SPECIMEN_BB. } 4 \\
\text { SIC_SPRING. } 7 \\
\text { GRAFOIL_SPACER. } 1 \\
\text { GRAFOIL_SPACER. } 2 \\
\text { GRAFOIL_SUPPORT. } 2\end{array}$ & $\begin{array}{l}\text { AL-6061 } \\
\text { AL-6061 } \\
\text { AL-6061 } \\
\text { GRAFOIL } \\
\text { Mo1y } \\
\text { SS304 } \\
\text { SiC(Irr) } \\
\text { Moly } \\
\text { F82H } \\
\text { SiC(Irr) } \\
\text { GRAFOIL } \\
\text { GRAFOIL } \\
\text { GRAFOIL }\end{array}$ & $\begin{array}{l}4.285 \\
0.527 \\
4.149 \\
0.004 \\
0.039 \\
2.457 \\
0.231 \\
0.039 \\
7.687 \\
0.097 \\
0.009 \\
0.009 \\
0.004\end{array}$ & $\begin{array}{r}59 . \\
82 . \\
172 . \\
235 . \\
235 . \\
218 . \\
232 . \\
233 . \\
236 . \\
218 . \\
163 . \\
155 . \\
234 .\end{array}$ & $\begin{array}{l}884 . \\
902 . \\
965 . \\
700 . \\
264 . \\
382 . \\
967 . \\
263 . \\
528 . \\
957 . \\
700 . \\
700 . \\
700 .\end{array}$ & $\begin{array}{r}149 . \\
29 . \\
609 . \\
1 . \\
2 . \\
186 . \\
47 . \\
2 . \\
878 . \\
18 . \\
1 . \\
1 . \\
1 .\end{array}$ \\
\hline & & & 19.537 & & & 1925. \\
\hline
\end{tabular}

RADIAL DIMENSIONS AND GAP SUMMARY FOR THE CORE-HOUSING GAP

CONTACT SUMMARY FOR CONTACT ID 25: Friction1esS - HOLDER TO HOUSING

Contact surface material:

Target surface material:

Interstitial gas:

Effective surface roughness:

Effective asperity slope:

Effective microhardness:
AL-6061

AL-6061

Helium

$2.263 \mu \mathrm{m}$

$0.214 \mathrm{rad}$

1.220 GPa

$\begin{array}{lrrr} & \text { Average } & \text { Minimum } & \text { Maximum } \\ \text { c direct results } & & & \\ \text { Contact status } & 1.000 & 1.000 & 1.000 \\ \text { Contact temperature }\left({ }^{\circ} \mathrm{C}\right) & 61.343 & 152.840 & 178.297 \\ \text { Target temperature }\left({ }^{\circ} \mathrm{C}\right) & 65.283 & 57.197 & 61.987 \\ \text { Geometric gas gap }(\mu \mathrm{m}) & 0.000 & 18.529 & 65.478 \\ \text { Contact pressure }(\mathrm{MPa}) & 367.136 & 300.000 & 0.000 \\ \text { Gap conduction heat flux }\left(\mathrm{kW} / \mathrm{m}^{2}\right) & 0.000 & 0.000 & 1695.468 \\ \text { Radiation heat flux }\left(\mathrm{kW} / \mathrm{m}^{2}\right) & & & 0.000\end{array}$




\begin{tabular}{|c|c|c|c|}
\hline $\begin{array}{l}\text { Contact conduction heat flux }\left(\mathrm{kW} / \mathrm{m}^{2}\right) \\
\text { Total heat flux }\left(\mathrm{kW} / \mathrm{m}^{2}\right) \\
\text { Therma1 contact conductance }\left(\mathrm{W} / \mathrm{m}^{2} \cdot \mathrm{C}\right)\end{array}$ & $\begin{array}{r}0.000 \\
367.136 \\
3362.074\end{array}$ & $\begin{array}{r}0.000 \\
300.871 \\
3159.420\end{array}$ & $\begin{array}{r}0.000 \\
1695.468 \\
16403.961\end{array}$ \\
\hline $\begin{array}{l}\text { N derlved results } \\
\text { Effective gas gap }(\mu \mathrm{m}) \\
\text { Contact therma1 jump distance }(\mu \mathrm{m}) \\
\text { Target thermal jump distance }(\mu \mathrm{m}) \\
\text { Effective contact pressure (MPa) } \\
\text { Pressure index } \\
\text { Gas thermal conductivity }\left(\mathrm{W} / \mathrm{m} \cdot{ }^{\circ} \mathrm{C}\right) \\
\text { solid spot conductance }\left(\mathrm{W} / \mathrm{m}^{2} \cdot \mathrm{C}\right) \\
\text { Gas gap conductance }\left(\mathrm{W} / \mathrm{m}^{2} \cdot \mathrm{C}\right)\end{array}$ & $\begin{array}{r}52.682 \\
1.110 \\
1.043 \\
0.000 \\
13.534 \\
0.182 \\
0.000 \\
3362.924\end{array}$ & $\begin{array}{r}8.878 \\
1.063 \\
1.005 \\
0.000 \\
13.534 \\
0.179 \\
0.000 \\
3158.830\end{array}$ & $\begin{array}{r}54.534 \\
1.130 \\
1.058 \\
0.000 \\
13.534 \\
0.184 \\
0.000 \\
16373.586\end{array}$ \\
\hline
\end{tabular}

Contact status codes:

$0=$ open/no heat transfer, $1=$ near-field contact

$2=$ closed and sliding, $\quad 3=c l o s e d$ and sticking 
$9.35 \mathrm{~mm}$ holder diameter (bend bar design)

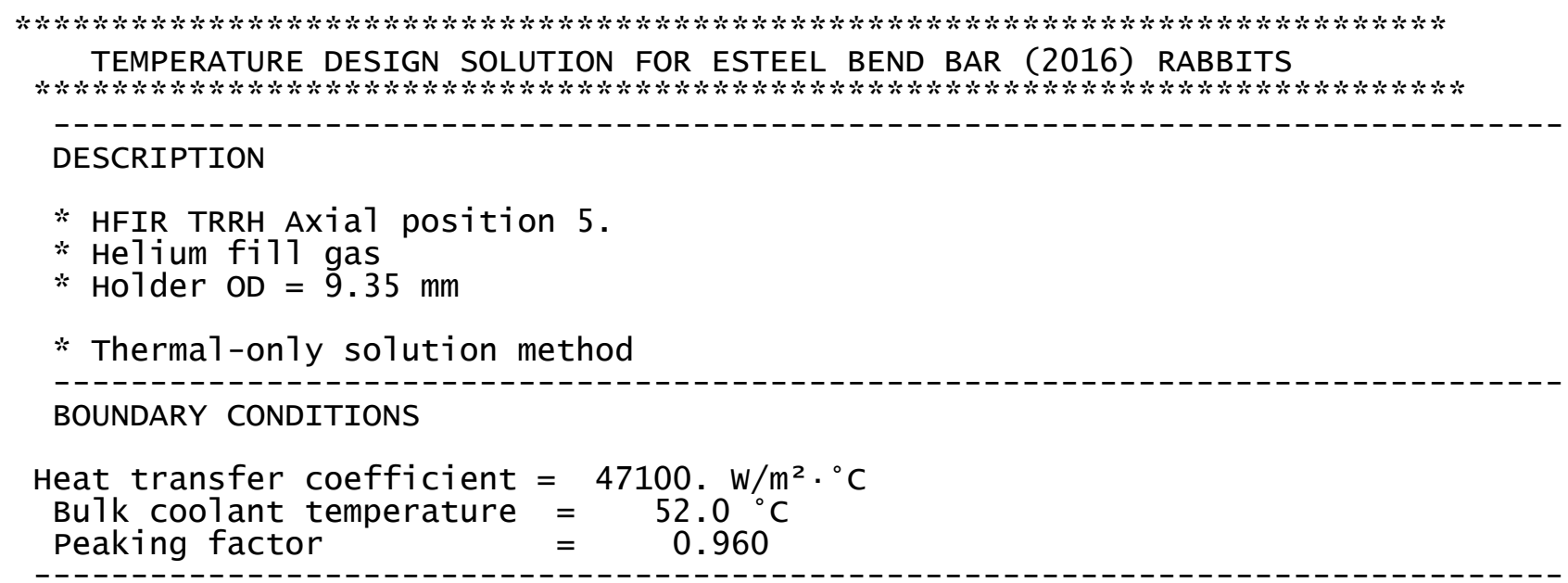

HEAT GENERATION

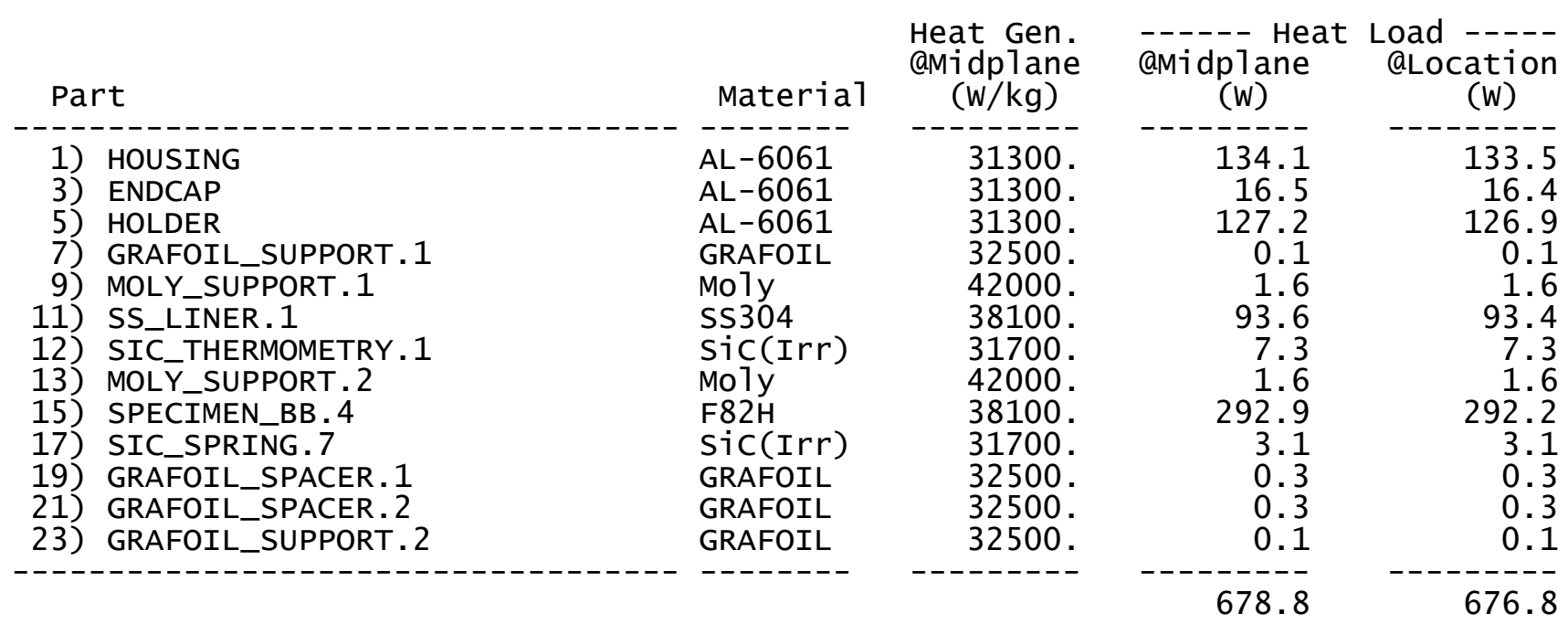

CAPSULE TEMPERATURE SUMMARY

\begin{tabular}{|c|c|c|c|c|c|c|c|}
\hline Name & & Material & Tavg & Tmin & Tmax & T. 025 & T. 975 \\
\hline $\begin{array}{r}\text { 1) } \\
3 \text { ) } \\
5 \text { ) } \\
7 \text { ) } \\
9 \text { ) } \\
11 \text { ) } \\
12) \\
13) \\
15) \\
17) \\
19) \\
21) \\
23)\end{array}$ & $\begin{array}{l}\text { HOUSING } \\
\text { ENDCAP } \\
\text { HOLDER } \\
\text { GRAFOIL_SUPPORT. } 1 \\
\text { MOLY_SUPPORT. } 1 \\
\text { SS_LINER.1 } \\
\text { SIC_THERMOMETRY.1 } \\
\text { MOLY_SUPPORT. } 2 \\
\text { SPECIMEN_BB. } 4 \\
\text { SIC_SPRING. } 7 \\
\text { GRAFOIL_SPACER.1 } \\
\text { GRAFOIL_SPACER. } 2 \\
\text { GRAFOIL_SUPPORT. } 2\end{array}$ & $\begin{array}{l}\text { AL-6061 } \\
\text { AL-6061 } \\
\text { AL-6061 } \\
\text { GRAFOIL } \\
\text { Mo1y } \\
\text { SS304 } \\
\text { SiC(Irr) } \\
\text { MO1y } \\
\text { F82H } \\
\text { SiC(Irr) } \\
\text { GRAFOIL } \\
\text { GRAFOIL } \\
\text { GRAFOIL }\end{array}$ & $\begin{array}{l}59 . \\
82 . \\
204 . \\
265 . \\
265 . \\
250 . \\
263 . \\
263 . \\
268 . \\
249 . \\
194 . \\
185 . \\
263 .\end{array}$ & $\begin{array}{r}54 . \\
80 . \\
182 . \\
260 . \\
259 . \\
219 . \\
253 . \\
256 . \\
237 . \\
228 . \\
174 . \\
169 . \\
256 .\end{array}$ & $\begin{array}{l}62 . \\
84 . \\
214 . \\
269 . \\
270 . \\
266 . \\
268 . \\
267 . \\
282 . \\
280 . \\
197 . \\
188 . \\
267 .\end{array}$ & $\begin{array}{l}55 . \\
81 . \\
186 . \\
261 . \\
260 . \\
234 . \\
254 . \\
258 . \\
249 . \\
230 . \\
184 . \\
177 . \\
260 .\end{array}$ & $\begin{array}{r}61 . \\
83 . \\
213 . \\
269 . \\
270 . \\
262 . \\
267 . \\
267 . \\
280 . \\
279 . \\
197 .\end{array}$ \\
\hline
\end{tabular}


Name

1) HOUSING
3) ENDCAP
5) HOLDER
7) GRAFOIL_SUPPORT . 1
9) MOLY_SUPPORT. 1
11) SS_LINER.1
12) SIC_THERMOMETRY . 1
13) MOLY_SUPPORT. 2
15) SPECIMEN_BB . 4
17) SIC_SPRING.7
19) GRAFOIL_SPACER. 1
21) GRAFOIL_SPACER. 2
23) GRAFOIL_SUPPORT. 2

\begin{tabular}{|c|c|c|c|}
\hline aterial & $\begin{array}{l}\text { Thermal } \\
\text { Cond. } \\
\left(\mathrm{w} / \mathrm{m} \cdot{ }^{\circ} \mathrm{C}\right)\end{array}$ & $\begin{array}{l}\text { Therma1 } \\
\text { Exp. } \\
\text { Coeff. } \\
\left(\mu \mathrm{m} / \mathrm{m} \cdot{ }^{\circ} \mathrm{C}\right)\end{array}$ & $\begin{array}{l}\text { Emis } \\
(---)\end{array}$ \\
\hline $\begin{array}{l}-6061 \\
-6061 \\
-6061 \\
\text {-6AFOIL } \\
\text { 77y } \\
3304 \\
\text { iC(Irr) } \\
\text { 37y } \\
32 \mathrm{H} \\
\text { iC(Irr) } \\
\text { RAFOIL }\end{array}$ & $\begin{array}{r}166.582 \\
169.241 \\
176.000 \\
38.000 \\
128.462 \\
18.719 \\
6.078 \\
128.561 \\
33.588 \\
6.083 \\
38.000 \\
38.000 \\
38.000\end{array}$ & $\begin{array}{r}24.21 \\
0.00 \\
24.69 \\
1.00 \\
0.00 \\
0.00 \\
3.15 \\
0.00 \\
11.14 \\
3.10 \\
1.00 \\
1.00 \\
1.00\end{array}$ & $\begin{array}{l}0.050 \\
0.050 \\
0.052 \\
0.500 \\
0.054 \\
0.143 \\
0.900 \\
0.054 \\
0.143 \\
0.900 \\
0.500 \\
0.500 \\
0.500\end{array}$ \\
\hline
\end{tabular}

STORED ENERGY SUMMARY AT THE AVERAGE PART TEMPERATURE

\begin{tabular}{|c|c|c|c|c|c|c|}
\hline Name & & Material & $\begin{array}{r}\text { Mass } \\
(\mathrm{g})\end{array}$ & $\begin{array}{l}\text { Tavg } \\
\left({ }^{\circ} \mathrm{C}\right)\end{array}$ & $\begin{array}{c}\text { Specific } \\
\text { Heat } \\
\left(\mathrm{J} / \mathrm{kg}^{\circ} \mathrm{C}\right)\end{array}$ & $\begin{array}{l}\text { Stored } \\
\text { Energy } \\
(\mathrm{J})\end{array}$ \\
\hline $\begin{array}{r}1) \\
3) \\
5) \\
7) \\
9) \\
11) \\
12) \\
13) \\
15) \\
17) \\
19) \\
21) \\
23)\end{array}$ & $\begin{array}{l}\text { HOUSING } \\
\text { ENDCAP } \\
\text { HOLDER } \\
\text { GRAFOIL_SUPPORT. } 1 \\
\text { MOLY_SUPPORT. } 1 \\
\text { SS_LINER.1 } \\
\text { SIC_THERMOMETRY.1 } \\
\text { MOLY_SUPPORT.2 } \\
\text { SPECIMEN_BB. } 4 \\
\text { SIC_SPRING. } 7 \\
\text { GRAFOIL_SPACER.1 } \\
\text { GRAFOIL_SPACER.2 } \\
\text { GRAFOIL_SUPPORT. } 2\end{array}$ & $\begin{array}{l}\text { AL-6061 } \\
\text { AL-6061 } \\
\text { AL-6061 } \\
\text { GRAFOIL } \\
\text { MO1y } \\
\text { SS304 } \\
\text { SiC(Irr) } \\
\text { MO1y } \\
\text { F82H } \\
\text { SiC(Irr) } \\
\text { GRAFOIL } \\
\text { GRAFOIL } \\
\text { GRAFOIL }\end{array}$ & $\begin{array}{l}4.286 \\
0.527 \\
4.063 \\
0.004 \\
0.039 \\
2.457 \\
0.231 \\
0.039 \\
7.687 \\
0.097 \\
0.009 \\
0.009 \\
0.004\end{array}$ & $\begin{array}{l}59 . \\
82 . \\
204 . \\
265 . \\
265 . \\
250 . \\
263 . \\
263 . \\
268 . \\
249 . \\
194 . \\
185 . \\
263 .\end{array}$ & $\begin{array}{l}884 . \\
902 . \\
985 . \\
700 . \\
265 . \\
384 . \\
991 . \\
265 . \\
537 . \\
981 . \\
700 . \\
700 . \\
700 .\end{array}$ & $\begin{array}{r}149 . \\
29 . \\
737 . \\
1 . \\
3 . \\
217 . \\
56 . \\
2 . \\
1023 . \\
22 . \\
1 . \\
1 . \\
1 .\end{array}$ \\
\hline & & & 19.451 & & & 2241. \\
\hline
\end{tabular}

RADIAL DIMENSIONS AND GAP SUMMARY FOR THE CORE-HOUSING GAP

CONTACT SUMMARY FOR CONTACT ID 25: Friction1esS - HOLDER TO HOUSING

Contact surface material:

Target surface material:

Interstitial gas:

Effective surface roughness:

Effective asperity slope:

Effective microhardness:
$A L-6061$

AL-6061

Helium

$2.263 \mu \mathrm{m}$

$0.214 \mathrm{rad}$

1.220 GPa

$\begin{array}{lrrr} & \text { Average } & \text { Minimum } & \text { Maximum } \\ \text { c direct results } & & & \\ \text { Contact status } & 1.000 & 1.000 & 1.000 \\ \text { Contact temperature }\left({ }^{\circ} \mathrm{C}\right) & 202.461 & 181.931 & 211.301 \\ \text { Target temperature }\left({ }^{\circ} \mathrm{C}\right) & 61.077 & 57.453 & 61.880 \\ \text { Geometric gas gap }(\mu \mathrm{m}) & 87.210 & 20.686 & 87.470 \\ \text { Contact pressure }(\mathrm{MPa}) & 0.000 & 0.000 & 0.000 \\ \text { Gap conduction heat flux }\left(\mathrm{kW} / \mathrm{m}^{2}\right) & 368.885 & 305.362 & 2226.203 \\ \text { Radiation heat flux }\left(\mathrm{kW} / \mathrm{m}^{2}\right) & 0.000 & 0.000 & 0.000\end{array}$




\begin{tabular}{|c|c|c|c|}
\hline $\begin{array}{l}\text { Contact conduction heat flux }\left(\mathrm{kW} / \mathrm{m}^{2}\right) \\
\text { Total heat flux }\left(\mathrm{kW} / \mathrm{m}^{2}\right) \\
\text { Thermal contact conductance }\left(\mathrm{W} / \mathrm{m}^{2} \cdot \mathrm{C}\right)\end{array}$ & $\begin{array}{r}0.000 \\
368.885 \\
2611.946\end{array}$ & $\begin{array}{r}0.000 \\
305.362 \\
2443.363\end{array}$ & $\begin{array}{r}0.000 \\
2226.203 \\
16694.596\end{array}$ \\
\hline $\begin{array}{l}\text { u derlved results } \\
\text { Effective gas gap }(\mu \mathrm{m}) \\
\text { Contact therma1 jump distance }(\mu \mathrm{m}) \\
\text { Target therma1 jump distance }(\mu \mathrm{m}) \\
\text { Effective contact pressure }(\mathrm{MPa}) \\
\text { Pressure index } \\
\text { Gas thermal conductivity }\left(\mathrm{W} / \mathrm{m} \cdot{ }^{\circ} \mathrm{C}\right) \\
\text { Solid spot conductance }\left(\mathrm{W} / \mathrm{m}^{2} \cdot \mathrm{C}\right) \\
\text { Gas gap conductance }\left(\mathrm{W} / \mathrm{m}^{2} \cdot \mathrm{C}\right)\end{array}$ & $\begin{array}{r}70.827 \\
1.186 \\
1.096 \\
0.000 \\
13.534 \\
0.188 \\
0.000 \\
2612.217\end{array}$ & $\begin{array}{r}8.878 \\
1.130 \\
1.053 \\
0.000 \\
13.534 \\
0.184 \\
0.000 \\
2442.627\end{array}$ & $\begin{array}{r}73.065 \\
1.209 \\
1.113 \\
0.000 \\
13.534 \\
0.189 \\
0.000 \\
16627.804\end{array}$ \\
\hline
\end{tabular}

Contact status codes:

$0=$ open/no heat transfer, $1=$ near-field contact

$2=$ closed and sliding, $\quad 3=c l o s e d$ and sticking 
$9.30 \mathrm{~mm}$ holder diameter (bend bar design)

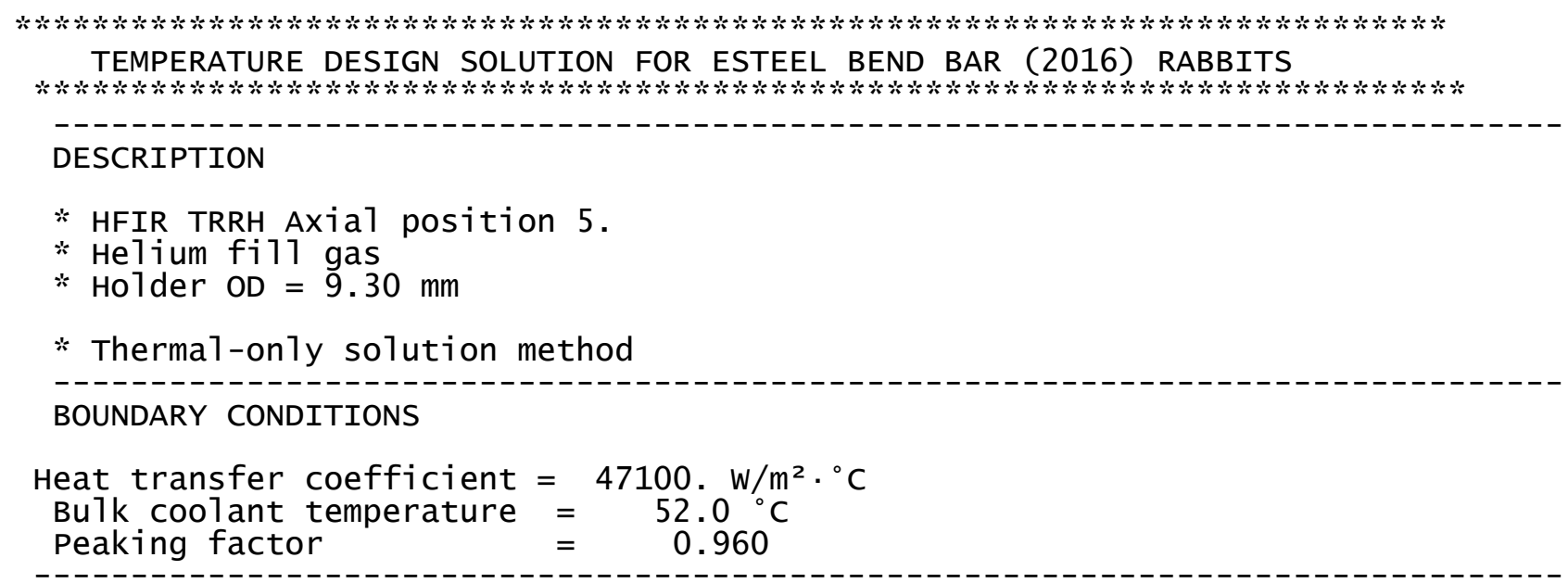

HEAT GENERATION

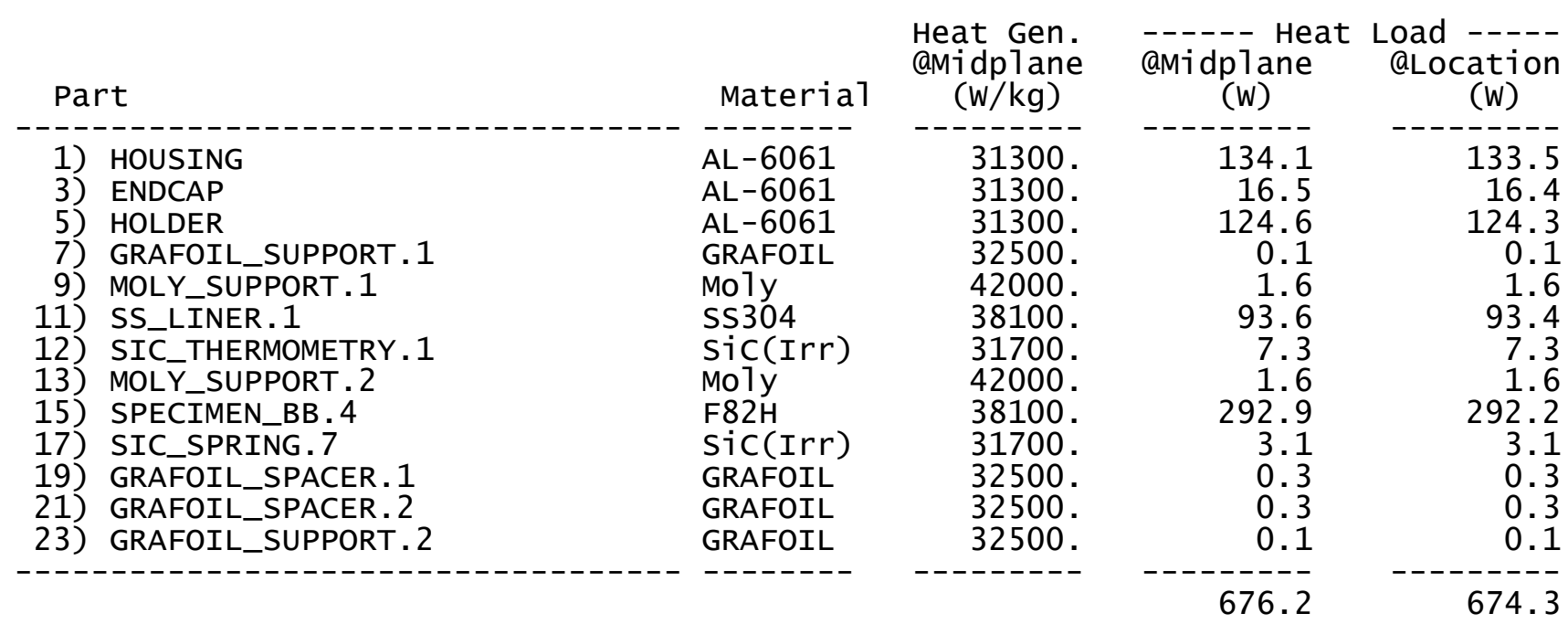

CAPSULE TEMPERATURE SUMMARY

\begin{tabular}{|c|c|c|c|c|c|c|c|}
\hline Name & & Material & Tavg & Tmin & Tmax & T. 025 & T. 975 \\
\hline $\begin{array}{r}1) \\
3) \\
5) \\
7) \\
9) \\
11) \\
12) \\
13) \\
15) \\
17) \\
19) \\
21) \\
23)\end{array}$ & $\begin{array}{l}\text { HOUSING } \\
\text { ENDCAP } \\
\text { HOLDER } \\
\text { GRAFOIL_SUPPORT. } 1 \\
\text { MOLY_SUPPORT. } 1 \\
\text { SS_LINER.1 } \\
\text { SIC_THERMOMETRY.1 } \\
\text { MOLY_SUPPORT. } 2 \\
\text { SPECIMEN_BB. } 4 \\
\text { SIC_SPRING. } 7 \\
\text { GRAFOIL_SPACER. } 1 \\
\text { GRAFOIL_SPACER.2 } \\
\text { GRAFOIL_SUPPORT. 2 }\end{array}$ & $\begin{array}{l}\text { AL-6061 } \\
\text { AL-6061 } \\
\text { AL-6061 } \\
\text { GRAFOIL } \\
\text { Mo1y } \\
\text { SS304 } \\
\text { SiC(Irr) } \\
\text { Mo1y } \\
\text { F82H } \\
\text { SiC(Irr) } \\
\text { GRAFOIL } \\
\text { GRAFOIL } \\
\text { GRAFOIL }\end{array}$ & $\begin{array}{l}59 . \\
82 . \\
233 . \\
293 . \\
293 . \\
278 . \\
291 . \\
290 . \\
296 . \\
278 . \\
222 . \\
211 . \\
290 .\end{array}$ & $\begin{array}{l}54 . \\
80 . \\
208 . \\
288 . \\
287 . \\
246 . \\
280 . \\
283 . \\
265 . \\
257 . \\
198 . \\
192 . \\
283 .\end{array}$ & $\begin{array}{l}62 . \\
84 . \\
244 . \\
297 . \\
298 . \\
295 . \\
297 . \\
294 . \\
311 . \\
308 . \\
225 . \\
215 . \\
294 .\end{array}$ & $\begin{array}{l}55 . \\
81 . \\
214 . \\
289 . \\
288 . \\
262 . \\
282 . \\
285 . \\
277 . \\
259 . \\
209 . \\
202 . \\
287 .\end{array}$ & $\begin{array}{r}61 . \\
84 . \\
242 . \\
297 . \\
298 . \\
291 .\end{array}$ \\
\hline
\end{tabular}


Name

1) HOUSING
3) ENDCAP
5) HOLDER
7) GRAFOIL_SUPPORT . 1
9) MOLY_SUPPORT. 1
11) SS_LINER. 1
12) SIC_THERMOMETRY . 1
13) MOLY_SUPPORT. 2
15) SPECIMEN_BB . 4
17) SIC_SPRING.7
19) GRAFOIL_SPACER. 1
21) GRAFOIL_SPACER. 2
23) GRAFOIL_SUPPORT . 2

\begin{tabular}{|c|c|c|c|}
\hline aterial & $\begin{array}{l}\text { Therma1 } \\
\text { cond. } \\
\left(\mathrm{W} / \mathrm{m} \cdot{ }^{\circ} \mathrm{C}\right)\end{array}$ & $\begin{array}{l}\text { Therma1 } \\
\text { Exp. } \\
\text { Coeff. } \\
\left(\mu \mathrm{m} / \mathrm{m} \cdot{ }^{\circ} \mathrm{C}\right)\end{array}$ & $\begin{array}{l}\text { Emis } \\
(---)\end{array}$ \\
\hline $\begin{array}{l}061 \\
061 \\
0 I L \\
4 \\
\text { Irr) } \\
\text { Irr) } \\
\text { OIL }\end{array}$ & $\begin{array}{r}166.580 \\
169.245 \\
176.000 \\
38.000 \\
127.342 \\
19.120 \\
6.067 \\
127.474 \\
33.739 \\
6.072 \\
38.000 \\
38.000 \\
38.000\end{array}$ & $\begin{array}{r}24.21 \\
0.00 \\
24.88 \\
1.00 \\
0.00 \\
0.00 \\
3.24 \\
0.00 \\
11.18 \\
3.20 \\
1.00 \\
1.00 \\
1.00\end{array}$ & $\begin{array}{l}0.050 \\
0.050 \\
0.053 \\
0.500 \\
0.057 \\
0.143 \\
0.900 \\
0.056 \\
0.143 \\
0.900 \\
0.500 \\
0.500 \\
0.500\end{array}$ \\
\hline
\end{tabular}

STORED ENERGY SUMMARY AT THE AVERAGE PART TEMPERATURE

\begin{tabular}{|c|c|c|c|c|c|c|}
\hline Name & & Material & $\begin{array}{r}\text { Mass } \\
\text { (g) }\end{array}$ & $\begin{array}{c}\text { Tavg } \\
\left({ }^{\circ} \mathrm{C}\right)\end{array}$ & $\begin{array}{l}\text { Specific } \\
\text { Heat } \\
\left(\mathrm{J} / \mathrm{kg}^{\circ} \mathrm{C}\right)\end{array}$ & $\begin{array}{l}\text { Stored } \\
\text { Energy } \\
\text { (J) }\end{array}$ \\
\hline $\begin{array}{r}1) \\
3) \\
5) \\
7) \\
9) \\
11) \\
12) \\
13) \\
15) \\
17) \\
19) \\
21) \\
23)\end{array}$ & $\begin{array}{l}\text { HOUSING } \\
\text { ENDCAP } \\
\text { HOLDER } \\
\text { GRAFOIL_SUPPORT.1 } \\
\text { MOLY_SUPPORT.1 } \\
\text { SS_LINER.1 } \\
\text { SIC_THERMOMETRY.1 } \\
\text { MOLY_SUPPORT.2 } \\
\text { SPECIMEN_BB. } 4 \\
\text { SIC_SPRING.7 } \\
\text { GRAFOIL_SPACER.1 } \\
\text { GRAFOIL_SPACER. } 2 \\
\text { GRAFOIL_SUPPORT. } 2\end{array}$ & $\begin{array}{l}\text { AL-6061 } \\
\text { AL-6061 } \\
\text { AL-6061 } \\
\text { GRAFOIL } \\
\text { MO7y } \\
\text { SS304 } \\
\text { SiC(Irr) } \\
\text { Mo1y } \\
\text { F82H } \\
\text { SiC(Irr) } \\
\text { GRAFOIL } \\
\text { GRAFOIL } \\
\text { GRAFOIL }\end{array}$ & $\begin{array}{l}4.286 \\
0.527 \\
3.982 \\
0.004 \\
0.039 \\
2.457 \\
0.231 \\
0.039 \\
7.687 \\
0.097 \\
0.009 \\
0.009 \\
0.004\end{array}$ & $\begin{array}{l}59 . \\
82 . \\
233 . \\
293 . \\
293 . \\
278 . \\
291 . \\
290 . \\
296 . \\
278 . \\
222 . \\
211 . \\
290 .\end{array}$ & $\begin{array}{r}884 . \\
902 . \\
998 . \\
700 . \\
266 . \\
386 . \\
1012 . \\
266 . \\
546 . \\
1002 . \\
700 . \\
700 . \\
700 .\end{array}$ & $\begin{array}{r}149 . \\
30 . \\
848 . \\
1 . \\
3 . \\
245 . \\
63 . \\
3 . \\
1160 . \\
25 . \\
1 . \\
1 . \\
1 .\end{array}$ \\
\hline & & & 19.370 & & & 2529. \\
\hline
\end{tabular}

RADIAL DIMENSIONS AND GAP SUMMARY FOR THE CORE-HOUSING GAP

CONTACT SUMMARY FOR CONTACT ID 25: Frictionless - HOLDER TO HOUSING

Contact surface material:

Target surface material:

Interstitial gas:

Effective surface roughness:

Effective asperity slope:

Effective microhardness:
$A L-6061$

AL-6061

Helium

$2.263 \mu \mathrm{m}$

$0.214 \mathrm{rad}$

1.220 GPa

$\begin{array}{lrrr} & \text { Average } & \text { Minimum } & \text { Maximum } \\ \text { c direct results } & & & \\ \text { Contact status } & 1.000 & 1.000 & 1.000 \\ \text { Contact temperature }\left({ }^{\circ} \mathrm{C}\right) & 231.637 & 208.259 & 241.323 \\ \text { Target temperature }\left({ }^{\circ} \mathrm{C}\right) & 61.041 & 57.576 & 61.793 \\ \text { Geometric gas gap }(\mu \mathrm{m}) & 108.139 & 23.601 & 108.475 \\ \text { Contact pressure }(\mathrm{MPa}) & 0.000 & 0.000 & 0.000 \\ \text { Gap conduction heat flux }\left(\mathrm{kW} / \mathrm{m}^{2}\right) & 370.065 & 306.628 & 2716.815 \\ \text { Radiation heat flux }\left(\mathrm{kW} / \mathrm{m}^{2}\right) & 0.000 & 0.000 & 0.000\end{array}$




\begin{tabular}{|c|c|c|c|}
\hline $\begin{array}{l}\text { Contact conduction heat flux }\left(\mathrm{kW} / \mathrm{m}^{2}\right) \\
\text { Total heat flux }\left(\mathrm{kW} / \mathrm{m}^{2}\right) \\
\text { Therma1 contact conductance }\left(\mathrm{W} / \mathrm{m}^{2} \cdot \mathrm{C}\right)\end{array}$ & $\begin{array}{r}0.000 \\
370.065 \\
2172.779\end{array}$ & $\begin{array}{r}0.000 \\
306.628 \\
2022.032\end{array}$ & $\begin{array}{r}0.000 \\
2716.815 \\
16948.385\end{array}$ \\
\hline $\begin{array}{l}\text { N derlved results } \\
\text { Effective gas gap }(\mu \mathrm{m}) \\
\text { Contact therma1 jump distance }(\mu \mathrm{m}) \\
\text { Target therma1 jump distance }(\mu \mathrm{m}) \\
\text { Effective contact pressure }(\mathrm{MPa}) \\
\text { Pressure index } \\
\text { Gas thermal conductivity }\left(\mathrm{W} / \mathrm{m} \cdot{ }^{\circ} \mathrm{C}\right) \\
\text { Solid spot conductance }\left(\mathrm{W} / \mathrm{m}^{2} \cdot \mathrm{C}\right) \\
\text { Gas gap conductance }\left(\mathrm{W} / \mathrm{m}^{2} \cdot \mathrm{C}\right)\end{array}$ & $\begin{array}{r}88.286 \\
1.256 \\
1.145 \\
0.000 \\
13.534 \\
0.193 \\
0.000 \\
2172.111\end{array}$ & $\begin{array}{r}8.878 \\
1.194 \\
1.098 \\
0.000 \\
13.534 \\
0.188 \\
0.000 \\
2021.650\end{array}$ & $\begin{array}{r}90.942 \\
1.281 \\
1.163 \\
0.000 \\
13.534 \\
0.194 \\
0.000 \\
16838.140\end{array}$ \\
\hline
\end{tabular}

Contact status codes:

$0=$ open/no heat transfer, $1=$ near-field contact

$2=$ closed and sliding, $\quad 3=c l o s e d$ and sticking 
$9.27 \mathrm{~mm}$ holder diameter (bend bar design)

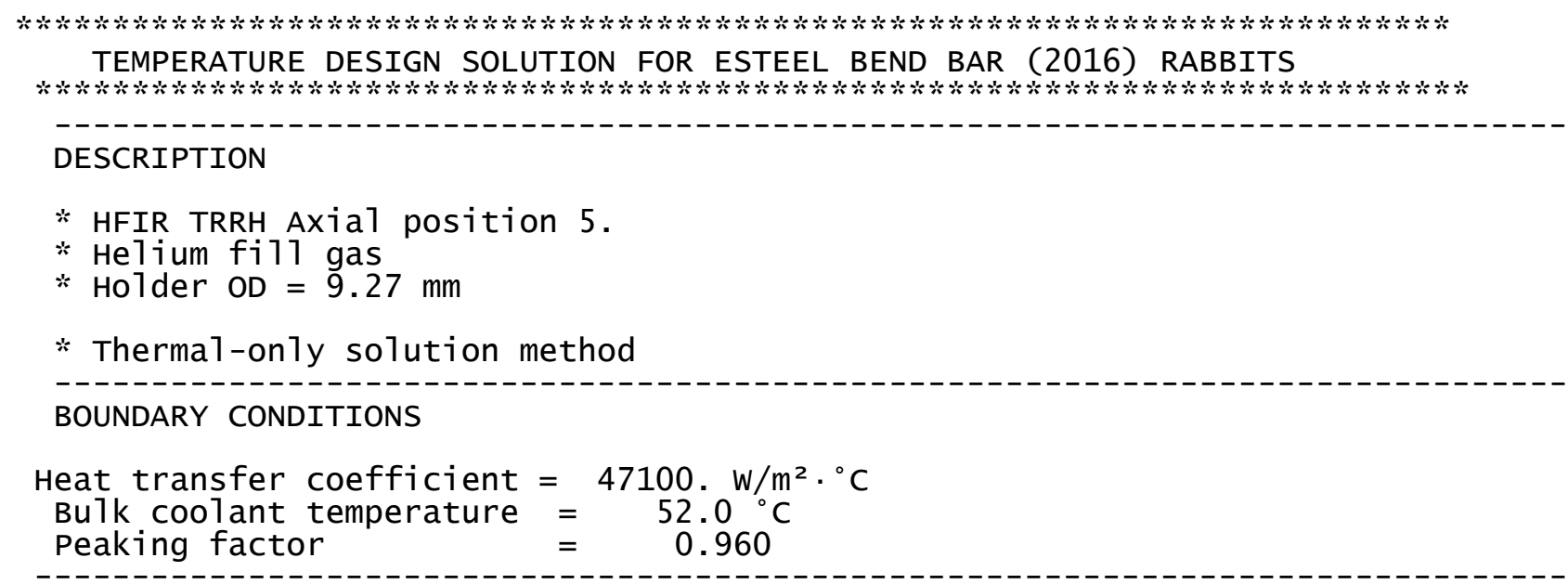

HEAT GENERATION

\begin{tabular}{|c|c|c|c|c|c|}
\hline Par & & Material & $\begin{array}{l}\text { Heat Gen. } \\
\text { amidplane } \\
\quad(\mathrm{w} / \mathrm{kg})\end{array}$ & $\begin{array}{c}\text { amidplane } \\
\text { (W) }\end{array}$ & $\begin{array}{c}\text { Load }---- \\
\text { aLocation } \\
\text { (W) }\end{array}$ \\
\hline $\begin{array}{l}\text { 1) } \\
3) \\
5) \\
7) \\
9) \\
11) \\
12) \\
13) \\
15) \\
17) \\
19) \\
21) \\
23)\end{array}$ & $\begin{array}{l}\text { HOUSING } \\
\text { ENDCAP } \\
\text { HOLDER } \\
\text { GRAFOIL_SUPPORT. } 1 \\
\text { MOLY_SUPPORT. } 1 \\
\text { SS_LINER.1 } \\
\text { SIC_THERMOMETRY.1 } \\
\text { MOLY_SUPPORT. } 2 \\
\text { SPECIMEN_BB. } 4 \\
\text { SIC_SPRING. } 7 \\
\text { GRAFOIL_SPACER.1 } \\
\text { GRAFOIL_SPACER. } 2 \\
\text { GRAFOIL_SUPPORT. } 2\end{array}$ & $\begin{array}{l}\text { AL-6061 } \\
\text { AL-6061 } \\
\text { AL-6061 } \\
\text { GRAFOIL } \\
\text { MO1y } \\
\text { SS304 } \\
\text { SiC(Irr) } \\
\text { MO1y } \\
\text { F82H } \\
\text { SiC(Irr) } \\
\text { GRAFOIL } \\
\text { GRAFOIL } \\
\text { GRAFOIL }\end{array}$ & $\begin{array}{l}31300 . \\
31300 . \\
31300 . \\
32500 . \\
42000 . \\
38100 . \\
31700 . \\
42000 . \\
38100 . \\
31700 . \\
32500 . \\
32500 . \\
32500 .\end{array}$ & $\begin{array}{r}134.1 \\
16.5 \\
122.3 \\
0.1 \\
1.6 \\
93.6 \\
7.3 \\
1.6 \\
292.9 \\
3.1 \\
0.3 \\
0.3 \\
0.1\end{array}$ & $\begin{array}{r}133.5 \\
16.4 \\
122.0 \\
0.1 \\
1.6 \\
93.4 \\
7.3 \\
1.6 \\
292.2 \\
3.1 \\
0.3 \\
0.3 \\
0.1\end{array}$ \\
\hline
\end{tabular}

CAPSULE TEMPERATURE SUMMARY

\begin{tabular}{|c|c|c|c|c|c|c|c|}
\hline Name & & Material & Tavg & Tmin & Tmax & Т. 025 & T. 975 \\
\hline $\begin{array}{l}\text { 1) } \\
3 \text { ) } \\
5 \text { ) } \\
7 \text { ) } \\
9 \text { ) } \\
11) \\
12) \\
13) \\
15) \\
17) \\
19) \\
21) \\
\text { 23) }\end{array}$ & $\begin{array}{l}\text { HOUSING } \\
\text { ENDCAP } \\
\text { HOLDER } \\
\text { GRAFOIL_SUPPORT.1 } \\
\text { MOLY_SUPPORT. } 1 \\
\text { SS_LINER.1 } \\
\text { SIC_THERMOMETRY.1 } \\
\text { MOLY_SUPPORT. } 2 \\
\text { SPECIMEN_BB. } 4 \\
\text { SIC_SPRING. } 7 \\
\text { GRAFOIL_SPACER. } 1 \\
\text { GRAFOIL_SPACER. } 2 \\
\text { GRAFOIL_SUPPORT. } 2\end{array}$ & $\begin{array}{l}\text { AL-6061 } \\
\text { AL-6061 } \\
\text { AL-6061 } \\
\text { GRAFOIL } \\
\text { Mo1y } \\
\text { SS304 } \\
\text { SiC(Irr) } \\
\text { Mo1y } \\
\text { F82H } \\
\text { SiC(Irr) } \\
\text { GRAFOIL } \\
\text { GRAFOIL } \\
\text { GRAFOIL }\end{array}$ & $\begin{array}{l}59 . \\
82 . \\
259 . \\
318 . \\
318 . \\
303 . \\
316 . \\
314 . \\
321 . \\
303 . \\
246 . \\
235 . \\
314 .\end{array}$ & $\begin{array}{l}54 . \\
80 . \\
231 . \\
312 . \\
311 . \\
270 . \\
304 . \\
307 . \\
289 . \\
282 . \\
218 . \\
212 . \\
307 .\end{array}$ & $\begin{array}{l}62 . \\
84 . \\
270 . \\
322 . \\
322 . \\
320 . \\
322 . \\
318 . \\
337 . \\
333 . \\
250 . \\
238 . \\
318 .\end{array}$ & $\begin{array}{r}55 . \\
81 . \\
238 . \\
314 . \\
313 . \\
287 . \\
306 . \\
308 . \\
301 . \\
284 .\end{array}$ & $\begin{array}{l}61 . \\
84 . \\
268 . \\
321 . \\
322 . \\
317 . \\
321 . \\
318 . \\
335 . \\
332 . \\
250 . \\
238 . \\
317 .\end{array}$ \\
\hline
\end{tabular}


Name

1) HOUSING
3) ENDCAP
5) HOLDER
7) GRAFOIL_SUPPORT . 1
9) MOLY_SUPPORT. 1
11) SS_LINER. 1
12) SIC_THERMOMETRY . 1
13) MOLY_SUPPORT. 2
15) SPECIMEN_BB . 4
17) SIC_SPRING.7
19) GRAFOIL_SPACER. 1
21) GRAFOIL_SPACER. 2
23) GRAFOIL_SUPPORT . 2

\begin{tabular}{|c|c|c|c|}
\hline aterial & $\begin{array}{l}\text { Thermal } \\
\text { Cond. } \\
\left(\mathrm{w} / \mathrm{m} \cdot{ }^{\circ} \mathrm{C}\right)\end{array}$ & $\begin{array}{l}\text { Therma1 } \\
\text { Exp. } \\
\text { Coeff. } \\
\left(\mu \mathrm{m} / \mathrm{m} \cdot{ }^{\circ} \mathrm{C}\right)\end{array}$ & $\begin{array}{l}\text { Emis } \\
(---)\end{array}$ \\
\hline $\begin{array}{l}-6061 \\
-6061 \\
-6061 \\
\text {-6AFOIL } \\
\text { J7y } \\
5304 \\
\text { iC(Irr) } \\
\text { icy } \\
32 \mathrm{H} \\
\text { iC(Irr) } \\
\text { RAFOIL }\end{array}$ & $\begin{array}{r}166.577 \\
169.246 \\
176.000 \\
38.000 \\
126.366 \\
19.470 \\
6.058 \\
126.522 \\
33.770 \\
6.063 \\
38.000 \\
38.000 \\
38.000\end{array}$ & $\begin{array}{r}24.21 \\
0.00 \\
25.09 \\
1.00 \\
0.00 \\
0.00 \\
3.31 \\
0.00 \\
11.30 \\
3.28 \\
1.00 \\
1.00 \\
1.00\end{array}$ & $\begin{array}{l}0.050 \\
0.050 \\
0.055 \\
0.500 \\
0.059 \\
0.143 \\
0.900 \\
0.059 \\
0.143 \\
0.900 \\
0.500 \\
0.500 \\
0.500\end{array}$ \\
\hline
\end{tabular}

STORED ENERGY SUMMARY AT THE AVERAGE PART TEMPERATURE

\begin{tabular}{|c|c|c|c|c|c|c|}
\hline Name & & Material & $\begin{array}{r}\text { Mass } \\
(\mathrm{g})\end{array}$ & $\begin{array}{l}\text { Tavg } \\
\left({ }^{\circ} \mathrm{C}\right)\end{array}$ & $\begin{array}{c}\text { Specific } \\
\text { Heat } \\
\left(\mathrm{J} / \mathrm{kg}^{\circ} \mathrm{C}\right)\end{array}$ & $\begin{array}{l}\text { Stored } \\
\text { Energy } \\
(\mathrm{J})\end{array}$ \\
\hline $\begin{array}{r}\text { 1) } \\
3) \\
5) \\
7) \\
9) \\
11) \\
12) \\
13) \\
15) \\
17) \\
19) \\
21) \\
23)\end{array}$ & $\begin{array}{l}\text { HOUSING } \\
\text { ENDCAP } \\
\text { HOLDER } \\
\text { GRAFOIL_SUPPORT.1 } \\
\text { MOLY_SUPPORT. } 1 \\
\text { SS_LINER.1 } \\
\text { SIC_THERMOMETRY.1 } \\
\text { MOLY_SUPPORT. } 2 \\
\text { SPECIMEN_BB. } 4 \\
\text { SIC_SPRING. } 7 \\
\text { GRAFOIL_SPACER.1 } \\
\text { GRAFOIL_SPACER. } 2 \\
\text { GRAFOIL_SUPPORT. } 2\end{array}$ & $\begin{array}{l}\text { AL-6061 } \\
\text { AL-6061 } \\
\text { AL-6061 } \\
\text { GRAFOIL } \\
\text { MO7y } \\
\text { SS304 } \\
\text { SiC(Irr) } \\
\text { Mo1y } \\
\text { F82H } \\
\text { SiC(Irr) } \\
\text { GRAFOIL } \\
\text { GRAFOIL } \\
\text { GRAFOIL }\end{array}$ & $\begin{array}{l}4.286 \\
0.527 \\
3.908 \\
0.004 \\
0.039 \\
2.457 \\
0.231 \\
0.039 \\
7.687 \\
0.097 \\
0.009 \\
0.009 \\
0.004\end{array}$ & $\begin{array}{r}59 . \\
82 . \\
259 . \\
318 . \\
318 . \\
303 . \\
316 . \\
314 . \\
321 . \\
303 . \\
246 . \\
235 . \\
314 .\end{array}$ & $\begin{array}{r}884 . \\
902 . \\
1010 . \\
700 . \\
267 . \\
388 . \\
1027 . \\
267 . \\
559 . \\
1020 . \\
700 . \\
700 . \\
700 .\end{array}$ & $\begin{array}{r}149 . \\
30 . \\
942 . \\
1 . \\
3 . \\
270 . \\
70 . \\
3 . \\
1294 . \\
28 . \\
1 . \\
1 . \\
1 .\end{array}$ \\
\hline & & & 19.296 & & & 2794 . \\
\hline
\end{tabular}

RADIAL DIMENSIONS AND GAP SUMMARY FOR THE CORE-HOUSING GAP

CONTACT SUMMARY FOR CONTACT ID 25: Friction1esS - HOLDER TO HOUSING

Contact surface material:

Target surface material:

Interstitial gas:

Effective surface roughness:

Effective asperity slope:

Effective microhardness:
AL-6061

AL-6061

Helium

$2.263 \mu \mathrm{m}$

$0.214 \mathrm{rad}$

1.220 GPa

$\begin{array}{lrrr} & \text { Average } & \text { Minimum } & \text { Maximum } \\ \text { c direct results } & & & \\ \text { Contact status } & 1.000 & 1.000 & 1.000 \\ \text { Contact temperature }\left({ }^{\circ} \mathrm{C}\right) & 257.003 & 230.947 & 267.426 \\ \text { Target temperature }\left({ }^{\circ} \mathrm{C}\right) & 61.008 & 57.650 & 61.722 \\ \text { Geometric gas gap }(\mu \mathrm{m}) & 127.084 & 26.988 & 127.464 \\ \text { Contact pressure }(\mathrm{MPa}) & 0.000 & 0.000 & 0.000 \\ \text { Gap conduction heat flux }\left(\mathrm{kW} / \mathrm{m}^{2}\right) & 370.928 & 307.426 & 3148.570 \\ \text { Radiation heat flux }\left(\mathrm{kW} / \mathrm{m}^{2}\right) & 0.000 & 0.000 & 0.000\end{array}$




\begin{tabular}{|c|c|c|c|}
\hline $\begin{array}{l}\text { Contact conduction heat flux }\left(\mathrm{kW} / \mathrm{m}^{2}\right) \\
\text { Total heat flux }\left(\mathrm{kW} / \mathrm{m}^{2}\right) \\
\text { Thermal contact conductance }\left(\mathrm{W} / \mathrm{m}^{2} \cdot \mathrm{C}\right)\end{array}$ & $\begin{array}{r}0.000 \\
370.928 \\
1896.574\end{array}$ & $\begin{array}{r}0.000 \\
307.426 \\
1757.606\end{array}$ & $\begin{array}{r}0.000 \\
3148.570 \\
17158.719\end{array}$ \\
\hline $\begin{array}{l}\text { Effective gas gap }(\mu \mathrm{m}) \\
\text { Contact therma1 jump distance }(\mu \mathrm{m}) \\
\text { Target therma1 jump distance }(\mu \mathrm{m}) \\
\text { Effective contact pressure }(\mathrm{MPa}) \\
\text { Pressure index } \\
\text { Gas thermal conductivity }\left(\mathrm{W} / \mathrm{m} \cdot{ }^{\circ} \mathrm{C}\right) \\
\text { Solid spot conductance }\left(\mathrm{W} / \mathrm{m}^{2} \cdot \mathrm{C}\right) \\
\text { Gas gap conductance }\left(\mathrm{W} / \mathrm{m}^{2} \cdot \mathrm{C}\right)\end{array}$ & $\begin{array}{r}104.162 \\
1.318 \\
1.187 \\
0.000 \\
13.534 \\
0.197 \\
0.000 \\
1895.731\end{array}$ & $\begin{array}{r}8.878 \\
1.250 \\
1.137 \\
0.000 \\
13.534 \\
0.192 \\
0.000 \\
1756.785\end{array}$ & $\begin{array}{r}107.180 \\
1.345 \\
1.207 \\
0.000 \\
13.534 \\
0.199 \\
0.000 \\
17064.052\end{array}$ \\
\hline
\end{tabular}

Contact status codes:

$0=$ open/no heat transfer, $1=$ near-field contact

$2=$ closed and sliding, $\quad 3=c l o s e d$ and sticking 
$9.23 \mathrm{~mm}$ holder diameter (bend bar design)

\begin{tabular}{|c|}
\hline 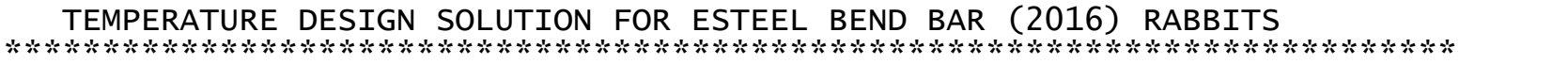 \\
\hline 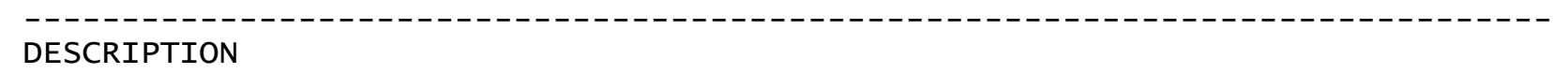 \\
\hline $\begin{array}{l}* \text { HFIR TRRH Axial position } 5 . \\
* \text { Helium fill gas } \\
* \text { Holder OD }=9.23 \mathrm{~mm}\end{array}$ \\
\hline * Thermal-only solution method \\
\hline BOUNDARY CONDITIONS \\
\hline $\begin{array}{rlc}\text { Heat transfer coefficient } & =47100 . \mathrm{W} / \mathrm{m}^{2} \cdot{ }^{\circ} \mathrm{C} \\
\text { Bulk coolant temperature } & =52.0{ }^{\circ} \mathrm{C} \\
\text { Peaking factor } & =0.960\end{array}$ \\
\hline
\end{tabular}

HEAT GENERATION

\begin{tabular}{|c|c|c|c|c|c|}
\hline Par & & Material & $\begin{array}{l}\text { Heat Gen. } \\
\text { amidplane } \\
\quad(\mathrm{w} / \mathrm{kg})\end{array}$ & $\begin{array}{c}\text { amidplane } \\
\text { (W) }\end{array}$ & $\begin{array}{l}\text { Load }---- \\
\text { aLocation } \\
\text { (W) }\end{array}$ \\
\hline $\begin{array}{l}\text { 1) } \\
3) \\
5) \\
7) \\
9) \\
11) \\
12) \\
13) \\
15) \\
17) \\
19) \\
21) \\
23)\end{array}$ & $\begin{array}{l}\text { HOUSING } \\
\text { ENDCAP } \\
\text { HOLDER } \\
\text { GRAFOIL_SUPPORT. } 1 \\
\text { MOLY_SUPPORT. } 1 \\
\text { SS_LINER.1 } \\
\text { SIC_THERMOMETRY.1 } \\
\text { MOLY_SUPPORT. } 2 \\
\text { SPECIMEN_BB. } 4 \\
\text { SIC_SPRING. } 7 \\
\text { GRAFOIL_SPACER.1 } \\
\text { GRAFOIL_SPACER. } 2 \\
\text { GRAFOIL_SUPPORT. } 2\end{array}$ & $\begin{array}{l}\text { AL-6061 } \\
\text { AL-6061 } \\
\text { AL-6061 } \\
\text { GRAFOIL } \\
\text { MO1y } \\
\text { SS304 } \\
\text { SiC(Irr) } \\
\text { MO1y } \\
\text { F82H } \\
\text { SiC(Irr) } \\
\text { GRAFOIL } \\
\text { GRAFOIL } \\
\text { GRAFOIL }\end{array}$ & $\begin{array}{l}31300 . \\
31300 . \\
31300 . \\
32500 . \\
42000 . \\
38100 . \\
31700 . \\
42000 . \\
38100 . \\
31700 . \\
32500 . \\
32500 . \\
32500 .\end{array}$ & $\begin{array}{r}134.1 \\
16.5 \\
119.9 \\
0.1 \\
1.6 \\
93.6 \\
7.3 \\
1.6 \\
292.9 \\
3.1 \\
0.3 \\
0.3 \\
0.1\end{array}$ & $\begin{array}{r}133.5 \\
16.4 \\
119.6 \\
0.1 \\
1.6 \\
93.4 \\
7.3 \\
1.6 \\
292.2 \\
3.1 \\
0.3 \\
0.3 \\
0.1\end{array}$ \\
\hline
\end{tabular}

CAPSULE TEMPERATURE SUMMARY

\begin{tabular}{|c|c|c|c|c|c|c|c|}
\hline Name & & Material & Tavg & Tmin & Tmax & T. 025 & T. 975 \\
\hline $\begin{array}{r}1) \\
3) \\
5) \\
7) \\
9) \\
11) \\
12) \\
13) \\
15) \\
17) \\
19) \\
21) \\
23)\end{array}$ & $\begin{array}{l}\text { HOUSING } \\
\text { ENDCAP } \\
\text { HOLDER } \\
\text { GRAFOIL_SUPPORT.1 } \\
\text { MOLY_SUPPORT. } 1 \\
\text { SS_LINER.1 } \\
\text { SIC_THERMOMETRY.1 } \\
\text { MOLY_SUPPORT. } 2 \\
\text { SPECIMEN_BB. } 4 \\
\text { SIC_SPRING. } 7 \\
\text { GRAFOIL_SPACER.1 } \\
\text { GRAFOIL_SPACER. } 2 \\
\text { GRAFOIL_SUPPORT. } 2\end{array}$ & $\begin{array}{l}\text { AL-6061 } \\
\text { AL-6061 } \\
\text { AL-6061 } \\
\text { GRAFOIL } \\
\text { Mo7y } \\
\text { SS304 } \\
\text { SiC(Irr) } \\
\text { Mo1y } \\
\text { F82H } \\
\text { SiC(Irr) } \\
\text { GRAFOIL } \\
\text { GRAFOIL } \\
\text { GRAFOIL }\end{array}$ & $\begin{array}{r}59 . \\
82 . \\
285 . \\
343 . \\
343 . \\
329 . \\
341 . \\
339 . \\
347 . \\
328 . \\
270 . \\
259 . \\
339 .\end{array}$ & $\begin{array}{l}54 . \\
80 . \\
255 . \\
338 . \\
336 . \\
295 . \\
328 . \\
331 . \\
313 . \\
308 . \\
239 . \\
232 . \\
331 .\end{array}$ & $\begin{array}{l}62 . \\
84 . \\
297 . \\
347 . \\
348 . \\
346 . \\
348 . \\
343 . \\
363 . \\
358 . \\
275 . \\
263 . \\
342 .\end{array}$ & $\begin{array}{r}55 . \\
81 . \\
262 . \\
339 . \\
338 . \\
312 . \\
330 . \\
333 . \\
326 . \\
310 . \\
254 . \\
246 . \\
336 .\end{array}$ & $\begin{array}{r}61 . \\
83 . \\
295 . \\
347 . \\
347 . \\
343 . \\
347 . \\
343 . \\
361 . \\
357 . \\
275 .\end{array}$ \\
\hline
\end{tabular}


Name

1) HOUSING
3) ENDCAP
5) HOLDER
7) GRAFOIL_SUPPORT . 1
9) MOLY_SUPPORT. 1
11) SS_LINER.1
12) SIC_THERMOMETRY . 1
13) MOLY_SUPPORT. 2
15) SPECIMEN_BB . 4
17) SIC_SPRING.7
19) GRAFOIL_SPACER. 1
21) GRAFOIL_SPACER. 2
23) GRAFOIL_SUPPORT. 2

\begin{tabular}{|c|c|c|c|}
\hline aterial & $\begin{array}{l}\text { Thermal } \\
\text { Cond. } \\
\left(\mathrm{w} / \mathrm{m} \cdot{ }^{\circ} \mathrm{C}\right)\end{array}$ & $\begin{array}{l}\text { Therma1 } \\
\text { Exp. } \\
\text { Coeff. } \\
\left(\mu \mathrm{m} / \mathrm{m} \cdot{ }^{\circ} \mathrm{C}\right)\end{array}$ & $\begin{array}{l}\text { Emis } \\
(---)\end{array}$ \\
\hline $\begin{array}{l}\text { L-6061 } \\
\text { L-6061 } \\
\text { RAFOIL } \\
\text { o1y } \\
\text { S304 } \\
\text { iC(Irr) } \\
\text { o1y } \\
82 \mathrm{H} \\
\text { iC(Irr) } \\
\text { RAFOIL }\end{array}$ & $\begin{array}{r}166.574 \\
169.243 \\
176.000 \\
38.000 \\
125.356 \\
19.829 \\
6.048 \\
125.538 \\
33.714 \\
6.053 \\
38.000 \\
38.000 \\
38.000\end{array}$ & $\begin{array}{r}24.21 \\
0.00 \\
25.29 \\
1.00 \\
0.00 \\
0.00 \\
3.38 \\
0.00 \\
11.42 \\
3.35 \\
1.00 \\
1.00 \\
1.00\end{array}$ & $\begin{array}{l}0.050 \\
0.050 \\
0.056 \\
0.500 \\
0.062 \\
0.143 \\
0.900 \\
0.061 \\
0.143 \\
0.900 \\
0.500 \\
0.500 \\
0.500\end{array}$ \\
\hline
\end{tabular}

STORED ENERGY SUMMARY AT THE AVERAGE PART TEMPERATURE

\begin{tabular}{|c|c|c|c|c|c|c|}
\hline Name & & Material & $\begin{array}{r}\text { Mass } \\
(\mathrm{g})\end{array}$ & $\begin{array}{l}\text { Tavg } \\
\left({ }^{\circ} \mathrm{C}\right)\end{array}$ & $\begin{array}{c}\text { Specific } \\
\text { Heat } \\
\left(\mathrm{J} / \mathrm{kg}^{\circ} \mathrm{C}\right)\end{array}$ & $\begin{array}{l}\text { Stored } \\
\text { Energy } \\
(\mathrm{J})\end{array}$ \\
\hline $\begin{array}{r}\text { 1) } \\
3) \\
5) \\
7) \\
9) \\
11) \\
12) \\
13) \\
15) \\
17) \\
19) \\
21) \\
23)\end{array}$ & $\begin{array}{l}\text { HOUSING } \\
\text { ENDCAP } \\
\text { HOLDER } \\
\text { GRAFOIL_SUPPORT.1 } \\
\text { MOLY_SUPPORT. } 1 \\
\text { SS_LINER.1 } \\
\text { SIC_THERMOMETRY.1 } \\
\text { MOLY_SUPPORT. } 2 \\
\text { SPECIMEN_BB. } 4 \\
\text { SIC_SPRING. } 7 \\
\text { GRAFOIL_SPACER.1 } \\
\text { GRAFOIL_SPACER. } 2 \\
\text { GRAFOIL_SUPPORT. } 2\end{array}$ & $\begin{array}{l}\text { AL-6061 } \\
\text { AL-6061 } \\
\text { AL-6061 } \\
\text { GRAFOIL } \\
\text { MO7y } \\
\text { SS304 } \\
\text { SiC(Irr) } \\
\text { Mo1y } \\
\text { F82H } \\
\text { SiC(Irr) } \\
\text { GRAFOIL } \\
\text { GRAFOIL } \\
\text { GRAFOIL }\end{array}$ & $\begin{array}{l}4.286 \\
0.527 \\
3.831 \\
0.004 \\
0.039 \\
2.457 \\
0.231 \\
0.039 \\
7.687 \\
0.097 \\
0.009 \\
0.009 \\
0.004\end{array}$ & $\begin{array}{r}59 . \\
82 . \\
285 . \\
343 . \\
343 . \\
329 . \\
341 . \\
339 . \\
347 . \\
328 . \\
270 . \\
259 . \\
339 .\end{array}$ & $\begin{array}{r}884 . \\
902 . \\
1022 . \\
700 . \\
268 . \\
390 . \\
1041 . \\
268 . \\
572 . \\
1034 . \\
700 . \\
700 . \\
700 .\end{array}$ & $\begin{array}{r}149 . \\
29 . \\
1036 . \\
1 . \\
3 . \\
296 . \\
77 . \\
3 . \\
1436 . \\
31 . \\
2 . \\
1 . \\
1 .\end{array}$ \\
\hline & & & 9.219 & & & 3067 \\
\hline
\end{tabular}

RADIAL DIMENSIONS AND GAP SUMMARY FOR THE CORE-HOUSING GAP

CONTACT SUMMARY FOR CONTACT ID 25: Friction1esS - HOLDER TO HOUSING

Contact surface material:

Target surface material:

Interstitial gas:

Effective surface roughness:

Effective asperity slope:

Effective microhardness:
AL-6061

AL-6061

Helium

$2.263 \mu \mathrm{m}$

$0.214 \mathrm{rad}$

1.220 GPa

$\begin{array}{lrrr} & \text { Average } & \text { Minimum } & \text { Maximum } \\ \text { c n direct results } & & & \\ \text { Contact status } & 1.000 & 1.000 & 1.000 \\ \text { Contact temperature }\left({ }^{\circ} \mathrm{C}\right) & 282.919 & 255.470 & 294.058 \\ \text { Target temperature }\left({ }^{\circ} \mathrm{C}\right) & 60.959 & 57.674 & 61.655 \\ \text { Geometric gas gap }(\mu \mathrm{m}) & 147.031 & 32.256 & 147.475 \\ \text { Contact pressure }(\mathrm{MPa}) & 0.000 & 0.000 & 0.000 \\ \text { Gap conduction heat flux }\left(\mathrm{kW} / \mathrm{m}^{2}\right) & 368.641 & 309.664 & 2669.851 \\ \text { Radiation heat flux }\left(\mathrm{kW} / \mathrm{m}^{2}\right) & 0.000 & 0.000 & 0.000\end{array}$




\begin{tabular}{|c|c|c|c|}
\hline $\begin{array}{l}\text { Contact conduction heat flux }\left(\mathrm{kW} / \mathrm{m}^{2}\right) \\
\text { Total heat flux }\left(\mathrm{kW} / \mathrm{m}^{2}\right) \\
\text { Thermal contact conductance }\left(\mathrm{W} / \mathrm{m}^{2} \cdot \mathrm{C}\right)\end{array}$ & $\begin{array}{r}0.000 \\
368.641 \\
1663.425\end{array}$ & $\begin{array}{r}0.000 \\
309.664 \\
1551.558\end{array}$ & $\begin{array}{r}0.000 \\
2669.851 \\
12804.875\end{array}$ \\
\hline $\begin{array}{l}\text { Effective gas gap }(\mu \mathrm{m}) \\
\text { Contact therma1 jump distance }(\mu \mathrm{m}) \\
\text { Target therma1 jump distance }(\mu \mathrm{m}) \\
\text { Effective contact pressure }(\mathrm{MPa}) \\
\text { Pressure index } \\
\text { Gas thermal conductivity }\left(\mathrm{W} / \mathrm{m} \cdot{ }^{\circ} \mathrm{C}\right) \\
\text { Solid spot conductance }\left(\mathrm{W} / \mathrm{m}^{2} \cdot \mathrm{C}\right) \\
\text { Gas gap conductance }\left(\mathrm{W} / \mathrm{m}^{2} \cdot \mathrm{C}\right)\end{array}$ & $\begin{array}{r}120.945 \\
1.382 \\
1.231 \\
0.000 \\
13.534 \\
0.201 \\
0.000 \\
1678.378\end{array}$ & $\begin{array}{r}8.878 \\
1.309 \\
1.178 \\
0.000 \\
13.534 \\
0.196 \\
0.000 \\
1550.318\end{array}$ & $\begin{array}{r}124.237 \\
1.411 \\
1.252 \\
0.000 \\
13.534 \\
0.203 \\
0.000 \\
17235.774\end{array}$ \\
\hline
\end{tabular}

Contact status codes:

$0=$ open/no heat transfer, $1=$ near-field contact

$2=$ closed and sliding, $\quad 3=c l o s e d$ and sticking 
$9.19 \mathrm{~mm}$ holder diameter (bend bar design)

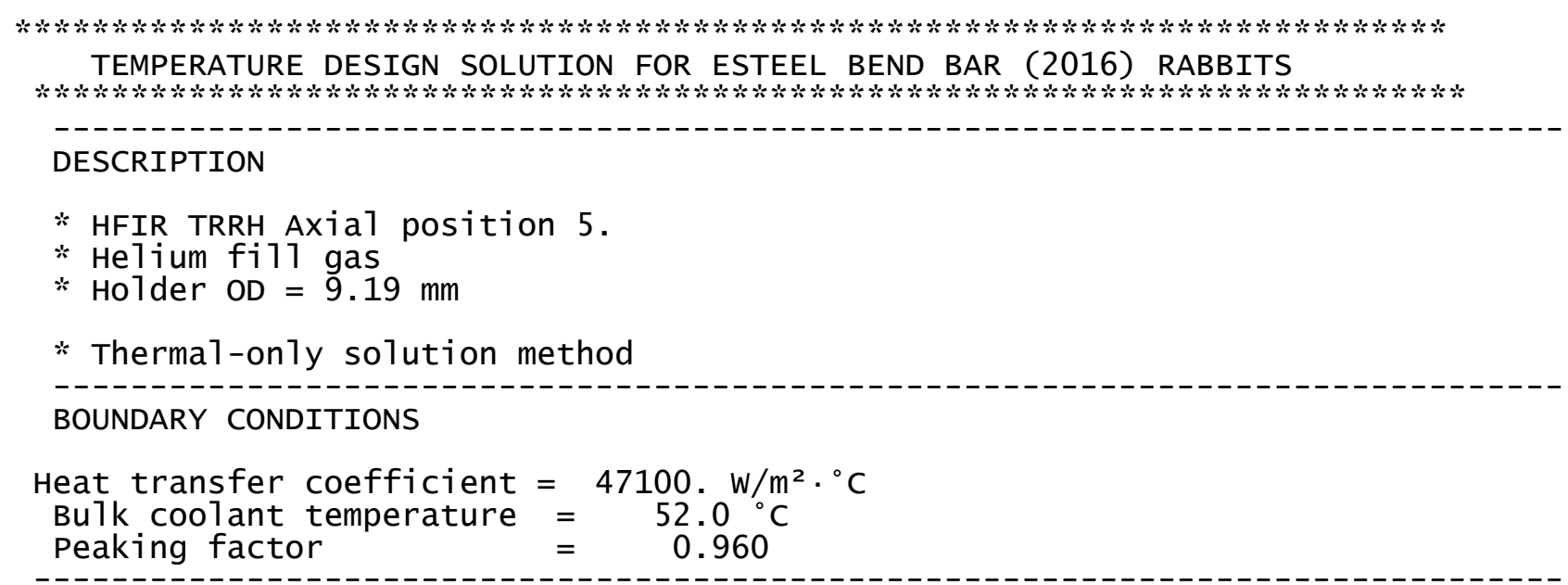

HEAT GENERATION

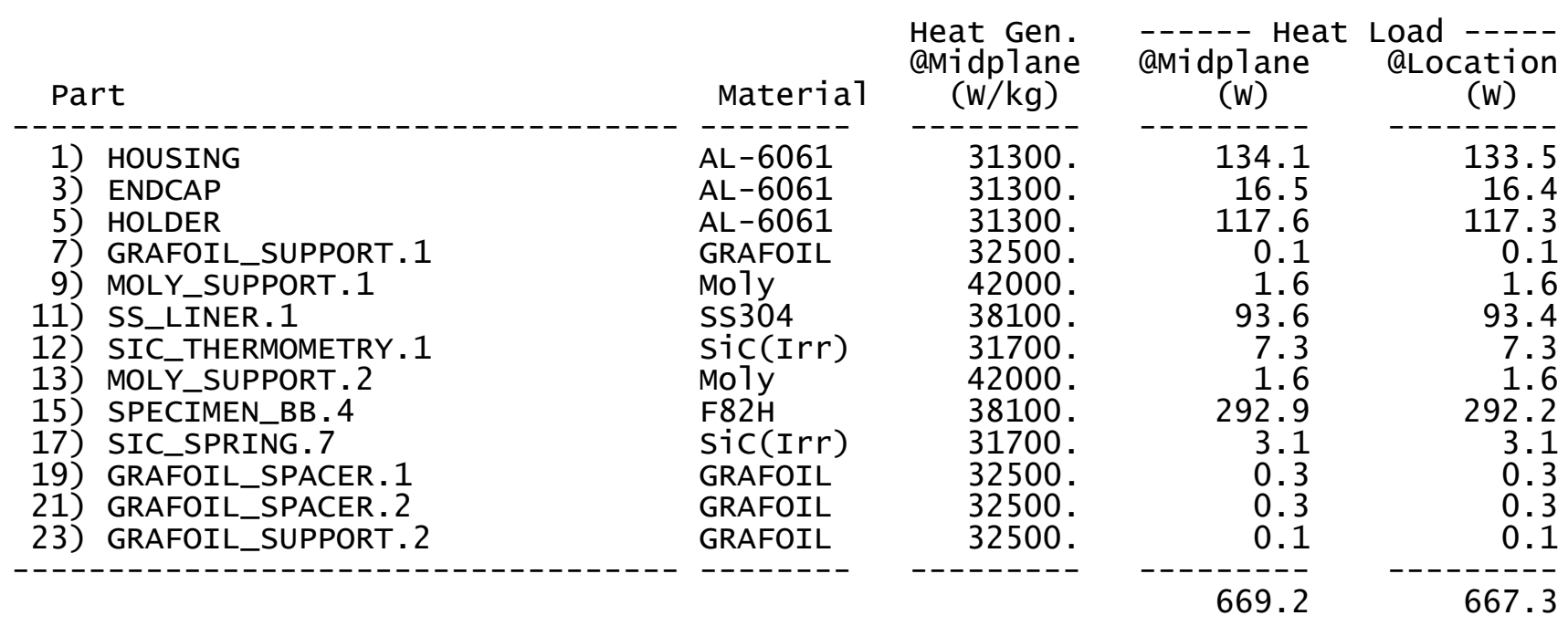

CAPSULE TEMPERATURE SUMMARY

\begin{tabular}{|c|c|c|c|c|c|c|c|}
\hline Name & & Material & Tavg & Tmin & Tmax & T. 025 & T. 975 \\
\hline $\begin{array}{l}\text { 1) } \\
3) \\
5) \\
7) \\
9) \\
11) \\
12) \\
13) \\
15) \\
17) \\
19) \\
21) \\
23)\end{array}$ & $\begin{array}{l}\text { HOUSING } \\
\text { ENDCAP } \\
\text { HOLDER } \\
\text { GRAFOIL_SUPPORT. } 1 \\
\text { MOLY_SUPPORT. } 1 \\
\text { SS_LINER.1 } \\
\text { SIC_THERMOMETRY.1 } \\
\text { MOLY_SUPPORT. } 2 \\
\text { SPECIMEN_BB. } 4 \\
\text { SIC_SPRING. } 7 \\
\text { GRAFOIL_SPACER.1 } \\
\text { GRAFOIL_SPACER.2 } \\
\text { GRAFOIL_SUPPORT. } 2\end{array}$ & $\begin{array}{l}\text { AL-6061 } \\
\text { AL-6061 } \\
\text { AL-6061 } \\
\text { GRAFOIL } \\
\text { Mo1y } \\
\text { SS304 } \\
\text { SiC(Irr) } \\
\text { Mo1y } \\
\text { F82H } \\
\text { SiC(Irr) } \\
\text { GRAFOIL } \\
\text { GRAFOIL } \\
\text { GRAFOIL }\end{array}$ & $\begin{array}{l}59 . \\
82 . \\
309 . \\
367 . \\
367 . \\
353 . \\
366 . \\
362 . \\
371 . \\
352 . \\
294 . \\
281 . \\
362 .\end{array}$ & $\begin{array}{l}54 . \\
80 . \\
277 . \\
361 . \\
360 . \\
318 . \\
352 . \\
354 . \\
337 . \\
332 . \\
258 . \\
251 . \\
354 .\end{array}$ & $\begin{array}{l}62 . \\
84 . \\
322 . \\
371 . \\
371 . \\
371 . \\
373 . \\
366 . \\
387 . \\
382 . \\
299 . \\
286 . \\
365 .\end{array}$ & $\begin{array}{r}55 . \\
81 . \\
286 . \\
362 . \\
361 . \\
335 . \\
353 . \\
356 . \\
350 . \\
334 . \\
275 . \\
266 . \\
359 .\end{array}$ & $\begin{array}{l}61 . \\
83 . \\
320 . \\
370 . \\
371 . \\
367 . \\
372 . \\
366 . \\
385 . \\
381 . \\
299 . \\
285 . \\
365 .\end{array}$ \\
\hline
\end{tabular}


Name

1) HOUSING
3) ENDCAP
5) HOLDER
7) GRAFOIL_SUPPORT . 1
9) MOLY_SUPPORT. 1
11) SS_LINER.1
12) SIC_THERMOMETRY . 1
13) MOLY_SUPPORT . 2
15) SPECIMEN_BB . 4
17) SIC_SPRING . 7
19) GRAFOIL_SPACER. 1
21) GRAFOIL_SPACER . 2
23) GRAFOIL_SUPPORT . 2

\begin{tabular}{|c|c|c|c|}
\hline aterial & $\begin{array}{l}\text { Therma1 } \\
\text { cond. } \\
\left(\mathrm{W} / \mathrm{m} \cdot{ }^{\circ} \mathrm{C}\right)\end{array}$ & $\begin{array}{l}\text { Therma1 } \\
\text { Exp. } \\
\text { Coeff. } \\
\left(\mu \mathrm{m} / \mathrm{m} \cdot{ }^{\circ} \mathrm{C}\right)\end{array}$ & $\begin{array}{l}\text { Emis } \\
(---)\end{array}$ \\
\hline $\begin{array}{l}-6061 \\
-6061 \\
\text { RAFOIL } \\
\text { 57y } \\
5304 \\
\text { ic(Irr) } \\
\text { j7y } \\
32 \mathrm{H} \\
\text { iC(Irr) } \\
\text { RAFOIL }\end{array}$ & $\begin{array}{r}166.572 \\
169.243 \\
176.000 \\
38.000 \\
124.406 \\
20.168 \\
6.039 \\
124.611 \\
33.661 \\
6.044 \\
38.000 \\
38.000 \\
38.000\end{array}$ & $\begin{array}{r}24.21 \\
0.00 \\
25.49 \\
1.00 \\
0.00 \\
0.00 \\
3.45 \\
0.00 \\
11.53 \\
3.41 \\
1.00 \\
1.00 \\
1.00\end{array}$ & $\begin{array}{l}0.050 \\
0.050 \\
0.057 \\
0.500 \\
0.064 \\
0.143 \\
0.900 \\
0.064 \\
0.143 \\
0.900 \\
0.500 \\
0.500 \\
0.500\end{array}$ \\
\hline
\end{tabular}

STORED ENERGY SUMMARY AT THE AVERAGE PART TEMPERATURE

\begin{tabular}{|c|c|c|c|c|c|c|}
\hline Name & & Material & $\begin{array}{c}\text { Mass } \\
(\mathrm{g})\end{array}$ & $\begin{array}{c}\text { Tavg } \\
\left({ }^{\circ} \mathrm{C}\right)\end{array}$ & $\begin{array}{l}\text { Specific } \\
\text { Heat } \\
\left(\mathrm{J} / \mathrm{kg}^{\circ} \mathrm{C}\right)\end{array}$ & $\begin{array}{l}\text { Stored } \\
\text { Energy } \\
\text { (J) }\end{array}$ \\
\hline $\begin{array}{l}\text { 1) } \\
3) \\
5) \\
7) \\
9) \\
11) \\
12) \\
13) \\
15) \\
17) \\
19) \\
21) \\
23)\end{array}$ & $\begin{array}{l}\text { HOUSING } \\
\text { ENDCAP } \\
\text { HOLDER } \\
\text { GRAFOIL_SUPPORT.1 } \\
\text { MOLY_SUPPORT. } 1 \\
\text { SS_LINER.1 } \\
\text { SIC_THERMOMETRY.1 } \\
\text { MOLY_SUPPORT. } 2 \\
\text { SPECIMEN_BB. } 4 \\
\text { SIC_SPRING.7 } \\
\text { GRAFOIL_SPACER.1 } \\
\text { GRAFOIL_SPACER. } 2 \\
\text { GRAFOIL_SUPPORT.2 }\end{array}$ & $\begin{array}{l}\text { AL-6061 } \\
\text { AL-6061 } \\
\text { AL-6061 } \\
\text { GRAFOIL } \\
\text { MO7y } \\
\text { SS304 } \\
\text { SiC(Irr) } \\
\text { MO1y } \\
\text { F82H } \\
\text { SiC(Irr) } \\
\text { GRAFOIL } \\
\text { GRAFOIL } \\
\text { GRAFOIL }\end{array}$ & $\begin{array}{l}4.286 \\
0.527 \\
3.756 \\
0.004 \\
0.039 \\
2.457 \\
0.231 \\
0.039 \\
7.687 \\
0.097 \\
0.009 \\
0.009 \\
0.004\end{array}$ & $\begin{array}{l}59 . \\
82 . \\
309 . \\
367 . \\
367 . \\
353 . \\
366 . \\
362 . \\
371 . \\
352 . \\
294 . \\
281 . \\
362 .\end{array}$ & $\begin{array}{r}884 . \\
902 . \\
1033 . \\
700 . \\
269 . \\
392 . \\
1054 . \\
269 . \\
582 . \\
1047 . \\
700 . \\
700 . \\
700 .\end{array}$ & $\begin{array}{r}149 . \\
29 . \\
1122 . \\
1 . \\
4 . \\
321 . \\
84 . \\
4 . \\
1570 . \\
34 . \\
2 . \\
2 . \\
1 .\end{array}$ \\
\hline & & & 19.144 & & & 3321. \\
\hline
\end{tabular}

RADIAL DIMENSIONS AND GAP SUMMARY FOR THE CORE-HOUSING GAP

CONTACT SUMMARY FOR CONTACT ID 25: Friction1esS - HOLDER TO HOUSING

Contact surface material:

Target surface material:

Interstitial gas:

Effective surface roughness:

Effective asperity slope:

Effective microhardness:
$A L-6061$

AL-6061

Helium

$2.263 \mu \mathrm{m}$

$0.214 \mathrm{rad}$

1.220 GPa

$\begin{array}{lrrr} & \text { Average } & \text { Minimum } & \text { Maximum } \\ \text { c n direct results } & & & \\ \text { Contact status } & 1.000 & 1.000 & 1.000 \\ \text { Contact temperature }\left({ }^{\circ} \mathrm{C}\right) & 307.287 & 277.646 & 319.192 \\ \text { Target temperature }\left({ }^{\circ} \mathrm{C}\right) & 60.920 & 57.740 & 61.593 \\ \text { Geometric gas gap }(\mu \mathrm{m}) & 166.485 & 37.880 & 166.961 \\ \text { Contact pressure }(\mathrm{MPa}) & 0.000 & 0.000 & 0.000 \\ \text { Gap conduction heat flux }\left(\mathrm{kW} / \mathrm{m}^{2}\right) & 368.154 & 310.204 & 2759.516 \\ \text { Radiation heat flux }\left(\mathrm{kW} / \mathrm{m}^{2}\right) & 0.000 & 0.000 & 0.000\end{array}$




\begin{tabular}{|c|c|c|c|}
\hline $\begin{array}{l}\text { Contact conduction heat flux }\left(\mathrm{kW} / \mathrm{m}^{2}\right) \\
\text { Total heat flux }\left(\mathrm{kW} / \mathrm{m}^{2}\right) \\
\text { Thermal contact conductance }\left(\mathrm{W} / \mathrm{m}^{2} \cdot \mathrm{C}\right)\end{array}$ & $\begin{array}{r}0.000 \\
368.154 \\
1496.825\end{array}$ & $\begin{array}{r}0.000 \\
310.204 \\
1395.309\end{array}$ & $\begin{array}{r}0.000 \\
2759.516 \\
11938.741\end{array}$ \\
\hline $\begin{array}{l}\text { Effective gas gap }(\mu \mathrm{m}) \\
\text { Contact therma1 jump distance }(\mu \mathrm{m}) \\
\text { Target therma1 jump distance }(\mu \mathrm{m}) \\
\text { Effective contact pressure }(\mathrm{MPa}) \\
\text { Pressure index } \\
\text { Gas thermal conductivity }\left(\mathrm{W} / \mathrm{m} \cdot{ }^{\circ} \mathrm{C}\right) \\
\text { Solid spot conductance }\left(\mathrm{W} / \mathrm{m}^{2} \cdot \mathrm{C}\right) \\
\text { Gas gap conductance }\left(\mathrm{W} / \mathrm{m}^{2} \cdot \mathrm{C}\right)\end{array}$ & $\begin{array}{r}137.414 \\
1.443 \\
1.272 \\
0.000 \\
13.534 \\
0.205 \\
0.000 \\
1504.668\end{array}$ & $\begin{array}{r}10.740 \\
1.364 \\
1.216 \\
0.000 \\
13.534 \\
0.200 \\
0.000 \\
1394.223\end{array}$ & $\begin{array}{r}140.972 \\
1.474 \\
1.294 \\
0.000 \\
13.534 \\
0.207 \\
0.000 \\
14984.520\end{array}$ \\
\hline
\end{tabular}

Contact status codes:

$0=$ open/no heat transfer, $1=$ near-field contact

$2=$ closed and sliding, $\quad 3=c l o s e d$ and sticking 


\section{ATTACHMENTS}




\begin{tabular}{|c|c|c|c|c|c|c|c|c|c|c|}
\hline \multirow{2}{*}{$\begin{array}{l}\text { Capsule Number: } \\
\text { Irradiation Conditions }\end{array}$} & \multicolumn{3}{|l|}{$\underline{\mathrm{ES}} 02$} & & & \multirow[b]{2}{*}{ Approvals } & & & & \\
\hline & \multirow{2}{*}{\multicolumn{3}{|c|}{ TRRH }} & & & & & & & \\
\hline Irradiation Location & & & & & & & Regue & & \multirow{2}{*}{\multicolumn{2}{|c|}{$5 / 418$}} \\
\hline Design Temperature & \multicolumn{4}{|c|}{4240} & & \multirow{3}{*}{$\begin{array}{l}\text { Checked by: } \\
\end{array}$} & & & & \\
\hline $\begin{array}{l}\text { First Cycle Goal } \\
\text { Irradiation Time }\end{array}$ & \multicolumn{4}{|c|}{$479.972 \pi n$} & & & & & & \\
\hline Irradiation Charge Number & & & N/A & & & & & & & \\
\hline Holder diameter & 9.10 & $\mathrm{~mm} / \mathrm{C}$ & 3583 in) at $20^{\circ} \mathrm{C}$ & & & & & & & \\
\hline Fill Gas & & & Helium & & & & & & & \\
\hline Capsule Fabrication & & & & & & & & & & \\
\hline & Drawing & Rev, & Part & Material & Count & Comment & MAT IR & FAB IR & ID & Mass (g) \\
\hline Housing & X3E020977A634 & A & 1 & Al 6061 & 1 & & 19348 & 20861 & SU2 & 4.9900 \\
\hline Housing end cap & X3E020977A634 & A & 2 & Al 4047 & 1 & & 20714 & 20714 & $17-29$ & 0.5150 \\
\hline Foil Spacer & S16-20-ESTEEL & 1 & 2 & Grafoil & 6 & & 19812 & 19812 & 6 Total & 0.0570 \\
\hline Wave Spring & S16-23-ESTEELB & 1 & CM08-L3-S17 & $17-7$ SS & 2 & & 20770 & 20770 & 2 Total & 0.2570 \\
\hline Specimen Holder & S16-22-ESTEEL & 1 & 3 & Al 6061 & 3 & & 20825 & 20784 & $17-05$ & 1.1341 \\
\hline & & & & & & & & & $17-06$ & 1.1353 \\
\hline & & & & & & & & & $17-07$ & 1.1412 \\
\hline \begin{tabular}{|l} 
Spring Pin \\
\end{tabular} & S16-21-ESTEEL & 2 & 91610A207 & $18-8 \mathrm{SS}$ & 3 & & 20548 & 20548 & 3 Total & 0.4130 \\
\hline Chevron & S16-18-FUSSAM01 & 1 & 1 & EuroFer & 18 & 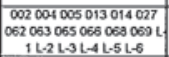 & 20777 & 20777 & 18 Total & 1.9633 \\
\hline Thermometry & S16-18-FUSSAM01 & 1 & 7 & SiC & 12 & & 19759 & 20840 & 13 & 0.1005 \\
\hline & & & & & & & & & 14 & 0.1001 \\
\hline & & & & & & & & & 15 & 0.0996 \\
\hline & & & & & & & & & 16 & 0.0997 \\
\hline & & & & & & & & & 17 & 0.0987 \\
\hline & & & & & & & & & $\frac{18}{19}$ & 0.1003 \\
\hline & & & & & & & & & $\frac{19}{20}$ & $\begin{array}{l}0.0995 \\
0.1005\end{array}$ \\
\hline & & & & & & & & & 21 & 0.0970 \\
\hline & & & & & & & & & 22 & 0.1002 \\
\hline & & & & & 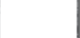 & & & & 23 & 0.0997 \\
\hline$\overline{S S J 3}$ & S16-18-FUSSAM01 & 1 & 2 & EuroFer & 20 & & 20779 & & 24 & 0.1002 \\
\hline & & & 2 & Eurorer & 20 & & 20778 & 20778 & E214 & 0.2604 \\
\hline & & & & & & & $\begin{array}{l}20778 \\
20778\end{array}$ & 20778 & E215 & 0.2611 \\
\hline & & & & & & & $\begin{array}{l}20778 \\
20778\end{array}$ & 20778 & $\begin{array}{l}\text { E216 } \\
\text { E217 }\end{array}$ & 0.2599 \\
\hline & & & & & & & 20778 & $\begin{array}{l}20778 \\
20778\end{array}$ & $\begin{array}{l}\text { E217 } \\
\text { E218 }\end{array}$ & 0.2590 \\
\hline & & & & & & & 20778 & 20778 & E219 & 0.2618 \\
\hline & & & & & & & 20778 & 20778 & E220 & 0.2613 \\
\hline & & & & & & & 20778 & 20778 & E221 & 0.2619 \\
\hline & & & & & & & 20778 & 20778 & E222 & 0.2604 \\
\hline & & & & & & & 20778 & 20778 & E223 & 0.2578 \\
\hline & & & & & & & 20778 & 20778 & E224 & 0.2644 \\
\hline & & & & & & & 20778 & 20778 & E225 & 0.2576 \\
\hline & & & & & & & 20841 & 20841 & YB06 & 0.2664 \\
\hline & & & & & 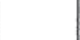 & & 20841 & 20841 & YB07 & 0.2665 \\
\hline & & & & & & & 20841 & 20841 & YB09 & 0.2628 \\
\hline & & & & & & & 20841 & 20841 & YB10 & 0.1749 \\
\hline & & & & & & & $\begin{array}{l}20841 \\
20841\end{array}$ & $\begin{array}{l}20841 \\
20841\end{array}$ & $\begin{array}{r}\text { YB11 } \\
\text { B54-02 }\end{array}$ & $\begin{array}{l}0.2747 \\
0.2674\end{array}$ \\
\hline MPC1 & S16-18-FUSSAM01 & 1 & 5 & EuroFer & 12 & & 20780 & 20780 & E306 & 0.1100 \\
\hline & & & & & & & & & E307 & 0.1110 \\
\hline & & & & & & & & & E308 & 0.1190 \\
\hline & & & & & & & & & $E 309$ & 0.1120 \\
\hline & & & & & & & & & E310 & 0.1140 \\
\hline MPC2 & S16-18-FUSSAM01 & 1 & 6 & FuroFer & 12 & & 20781 & 20781 & E311 & 0.1060 \\
\hline & & 1 & & Eutoret & 12 & & 20181 & $20 / 81$ & $\begin{array}{l}E 406 \\
\text { E407 }\end{array}$ & $\begin{array}{l}0.2610 \\
0.2560\end{array}$ \\
\hline & & & & & & & & & E408 & 0.2580 \\
\hline & & & & & & & & & E409 & 0.2580 \\
\hline & & & & & & & & & E410 & 0.2600 \\
\hline & & & & & & & & & E411 & 0.2590 \\
\hline & & & & & & & & & $\begin{array}{r}\text { Total Mass } \\
\text { Specimen Mass }\end{array}$ & \\
\hline Assembly & & & & & & & & & $\begin{array}{r}\text { Specimen Mass } \\
\text { Internal Mass }\end{array}$ & $\frac{6.8581}{14.1550}$ \\
\hline & Drawing & Rev. & Com & & & & & & & \\
\hline Assembly Drawing & S16-20-ESTEEL & 1 & & & & & & & & \\
\hline Welding \& Cleaning & X3E020977A633 & 0 & & & & & & & & \\
\hline Fill Gas & Helium & & & & & & & & & \\
\hline
\end{tabular}


ESO2

(17-05)

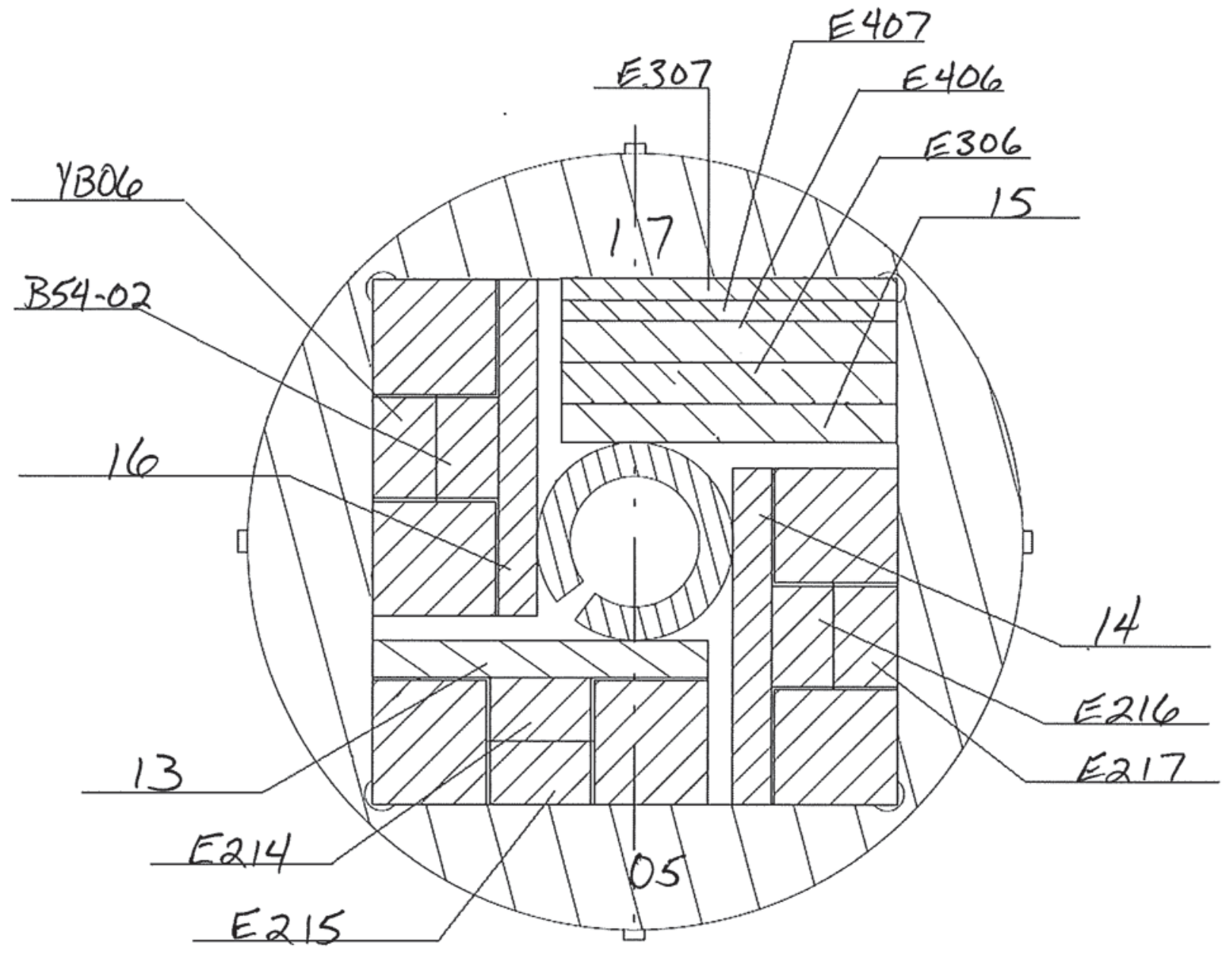


ES02

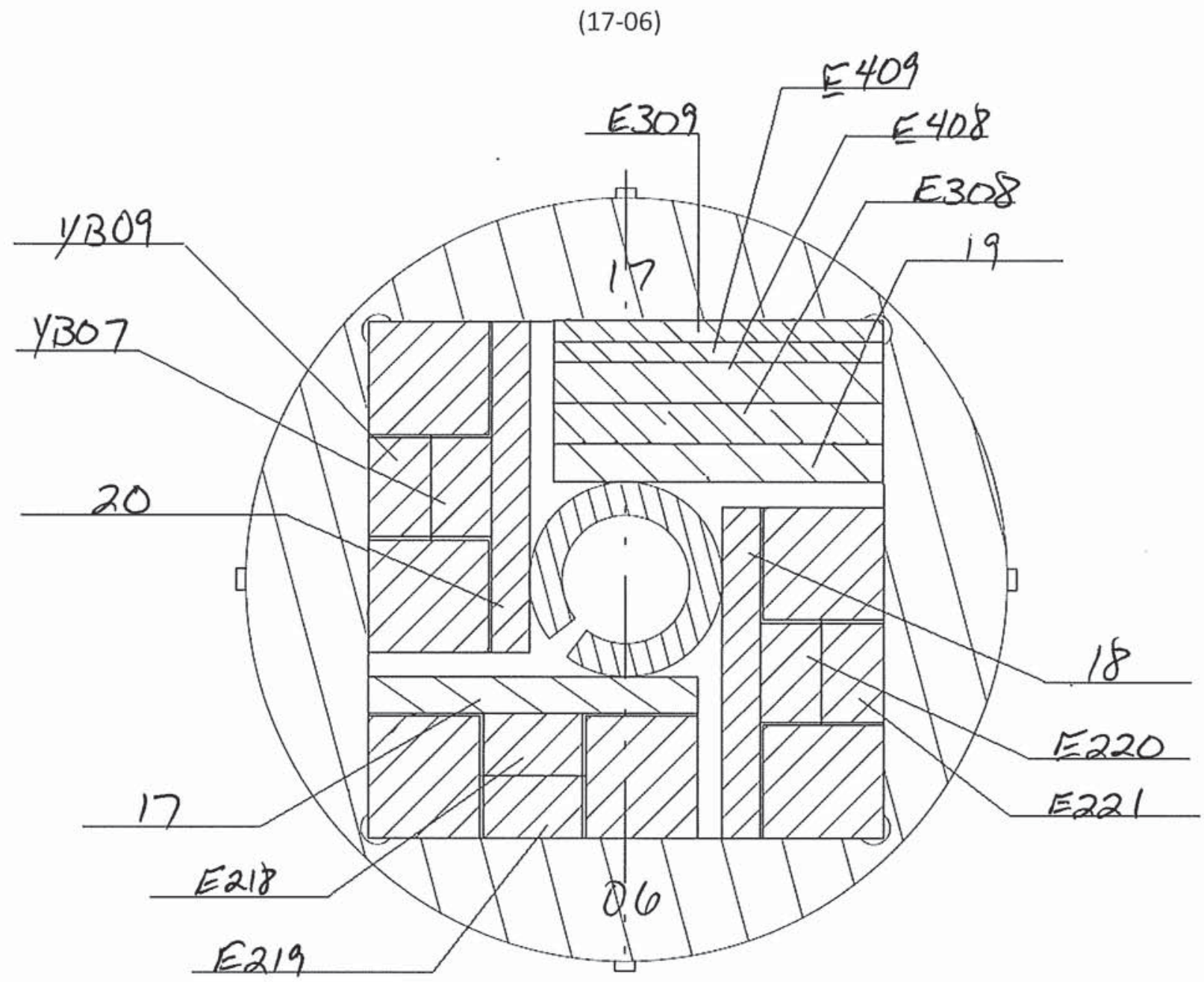


ES02

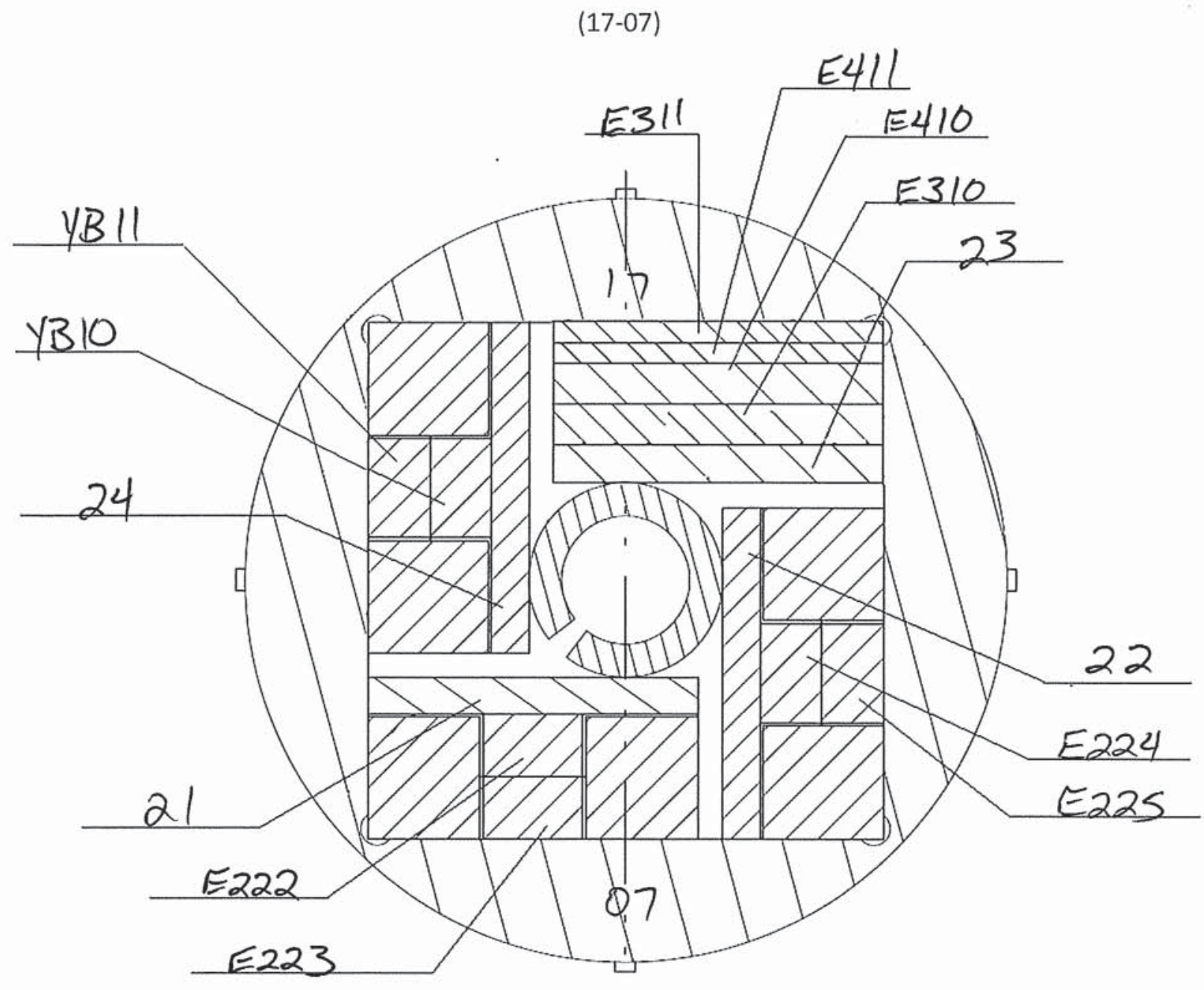




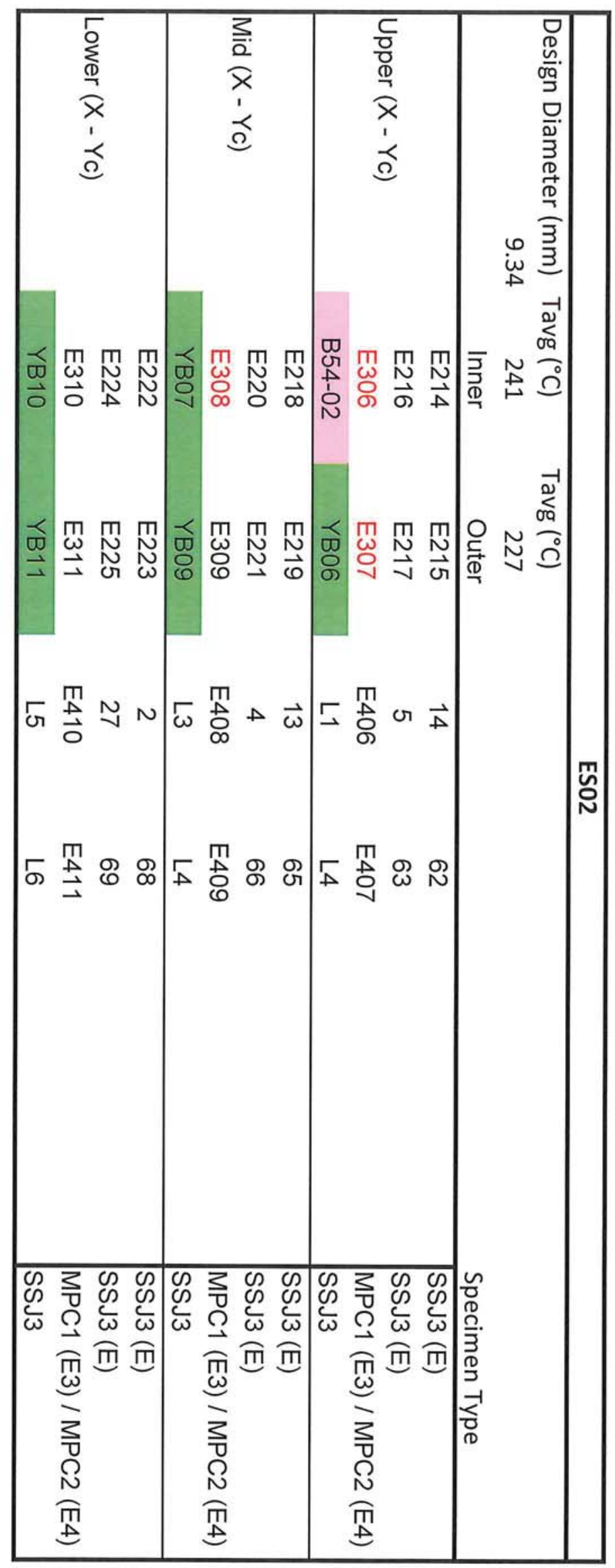




\section{RABBIT ASSEMBLY: ES $\varnothing 2$ HOUSING}

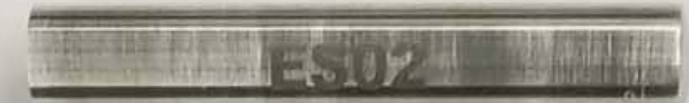

SN / IR NUMBER:

Su2/20861

INNER

DIAMETER:

$\varnothing 9.17 \mathrm{~mm}$

\section{HOLDER(S)}
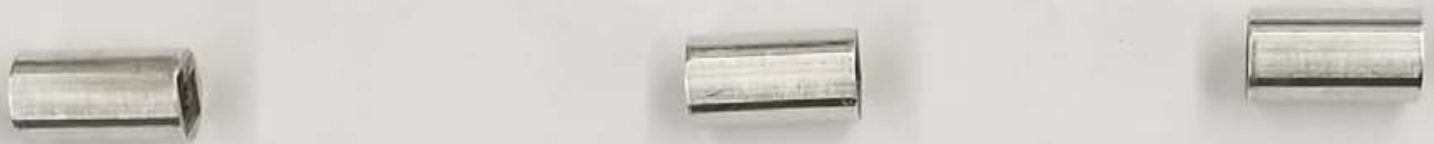

SN / IR NUMBER:

SN / IR NUMBER:

SN / IR NUMBER:

$17-\not 85 / 2 \varnothing 784$

$$
17-\not 66 / 28784
$$

$17-\varnothing 7 / 2 \varnothing 784$

OUTER

DIAMETER:

$\phi 9.1 \phi_{\mathrm{mm}}$
OUTER

DIAMETER:

$\phi 9.11 \mathrm{~mm}$
OUTER

DIAMETER:

$\varnothing 9.1 \varnothing$ 
Capsule Number:

Irradiation Conditions

Irradiation Location

Design Temperature

First Cycle Goal

Irradiation Time

Irradiation Charge Number

Holder diameter

Fill Gas

Capsule Fabrication

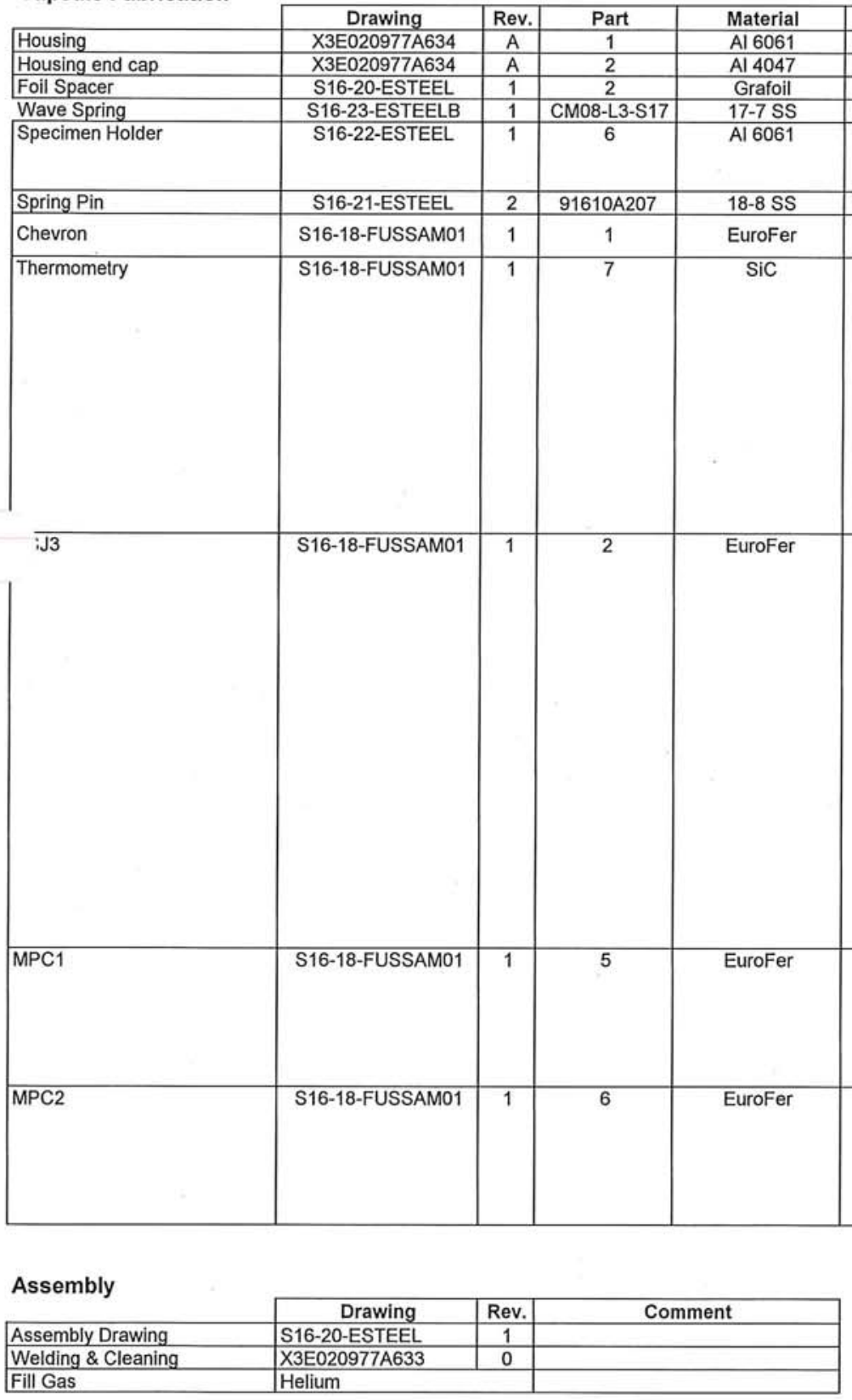

\begin{tabular}{rr} 
TRRH & 5 \\
\hline & 275 \\
\hline & 477 \\
\hline 2 & cyc. \\
\hline & N/A
\end{tabular}

9.20

$\mathrm{mm}\left(0.3622\right.$ in) at $20^{\circ} \mathrm{C}$ Helium

Approvals

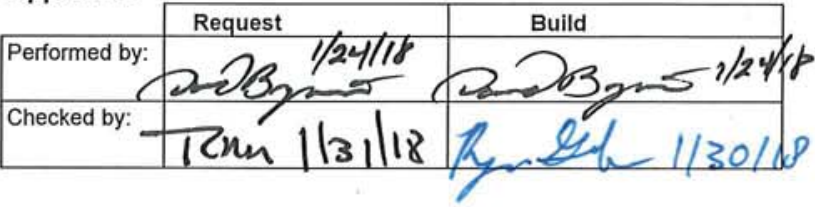

\begin{tabular}{l|l} 
Count & Comment \\
\hline
\end{tabular}

\begin{tabular}{|c|r|r|r|}
\hline MAT IR & FAB IR & ID & Mass $(\mathrm{g})$ \\
\hline 20838 & 20839 & $18-07$ & 4.2826 \\
\hline 20714 & 20714 & $17-32$ & 0.5135 \\
\hline 19812 & 19812 & 6 Total & 0.0560 \\
\hline 20770 & 20770 & 2 Total & 0.2560 \\
\hline 20825 & 20787 & $17-06$ & 1.2690 \\
\cline { 3 - 4 } & & $17-07$ & 1.2697 \\
\cline { 3 - 4 } & & $17-08$ & 1.2721 \\
\hline 20548 & 20548 & 3 Total & 0.4130 \\
\hline 20777 & 20777 & & 1.9687 \\
\hline
\end{tabular}

12

19759

\begin{tabular}{l|l|l|l}
12 & & & \\
\hline 12759 & 20840
\end{tabular}

20


Capsule Number: Irradiation Conditions Irradiation Location Design Temperature First Cycle Goal Irradiation Time Irradiation Charge Number Holder diameter Fill Gas ESO3

Capsule Fabrication

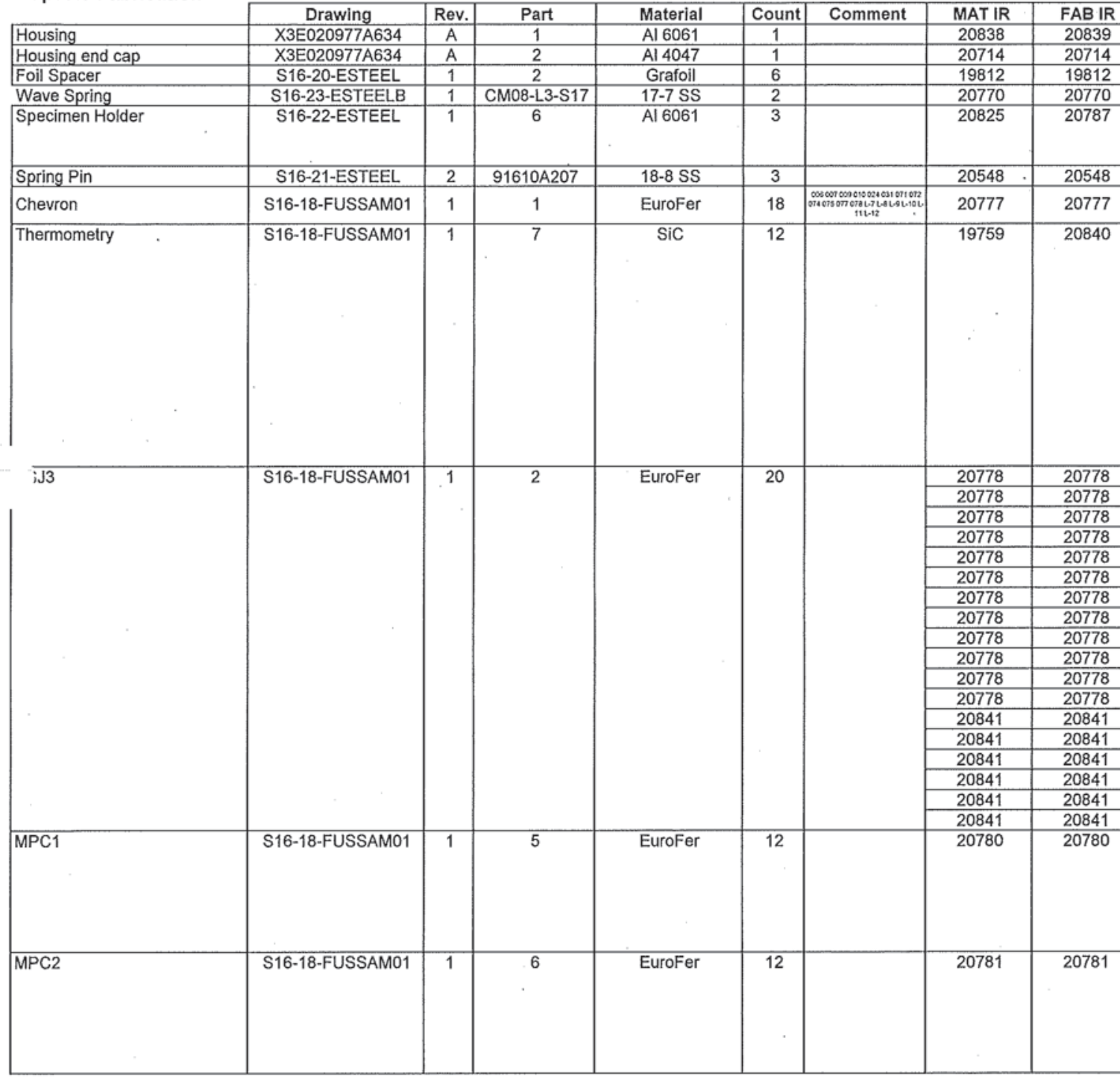

\section{Assembly}

\begin{tabular}{|l|l|c|c|}
\cline { 2 - 4 } \multicolumn{1}{c|}{} & \multicolumn{1}{c|}{ Drawing } & Rev. & Comment \\
\hline Assembly Drawing & S16-20-ESTEEL & 1 & \\
\hline Welding \& Cleaning & X3E020977A633 & 0 & \\
\hline Fill Gas & Helium & & \\
\hline
\end{tabular}

\begin{tabular}{rr} 
& TRRH \\
\hline & 275 \\
\hline & 477 \\
\hline 2 & cyc. \\
\hline & N/A \\
\hline $9.20 \quad \mathrm{~mm}(0.3622$ in $)$ at $20^{\circ} \mathrm{C}$ \\
\hline
\end{tabular}
Helium

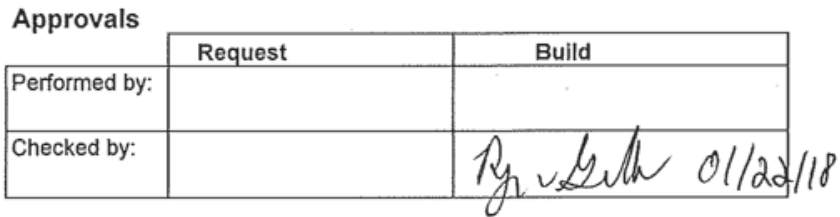

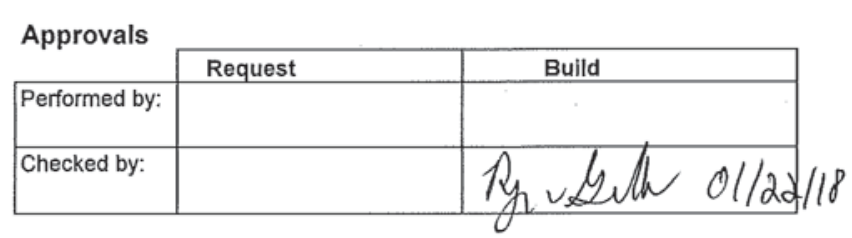


ES03

(17-06)

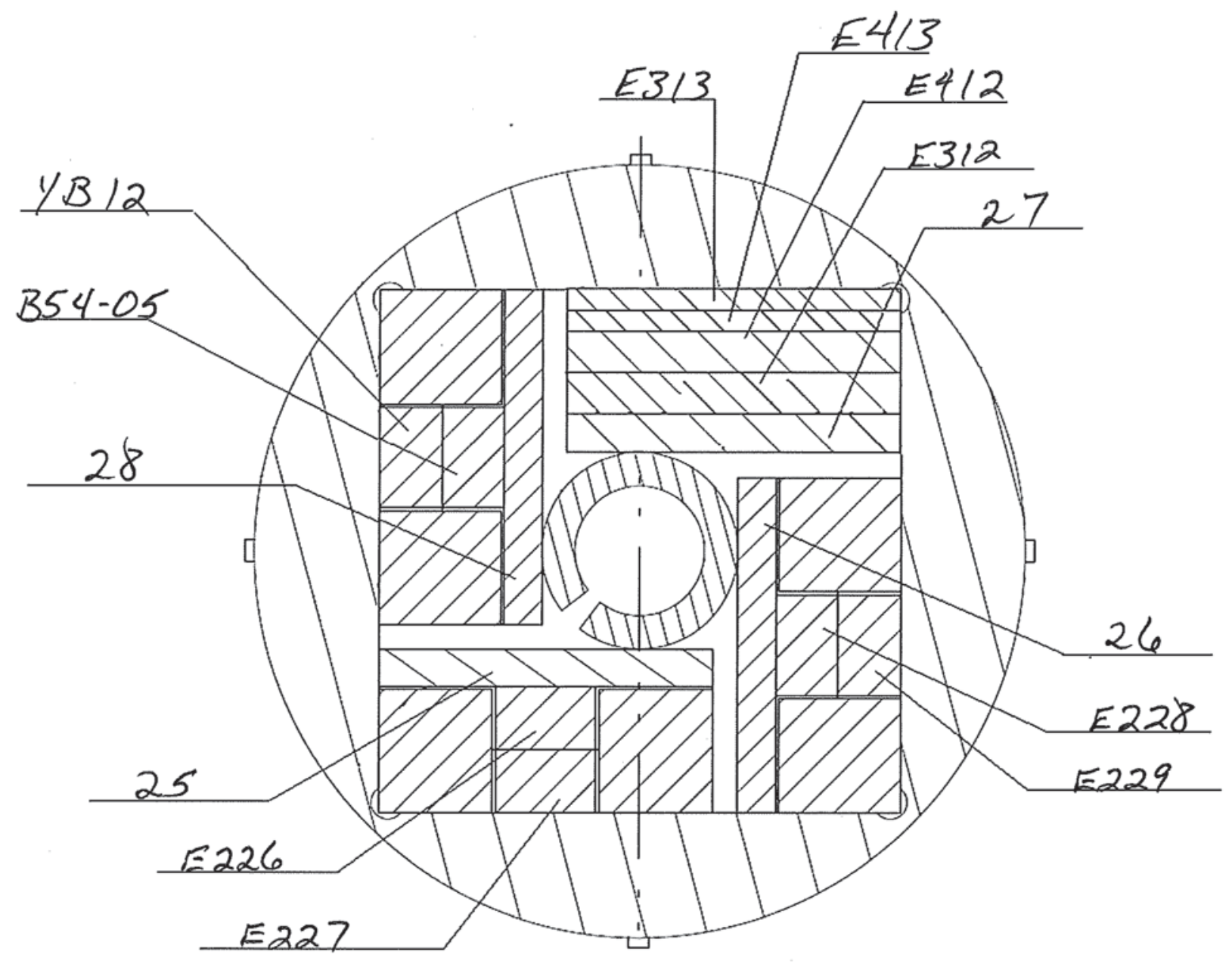


ES03

(17-07)

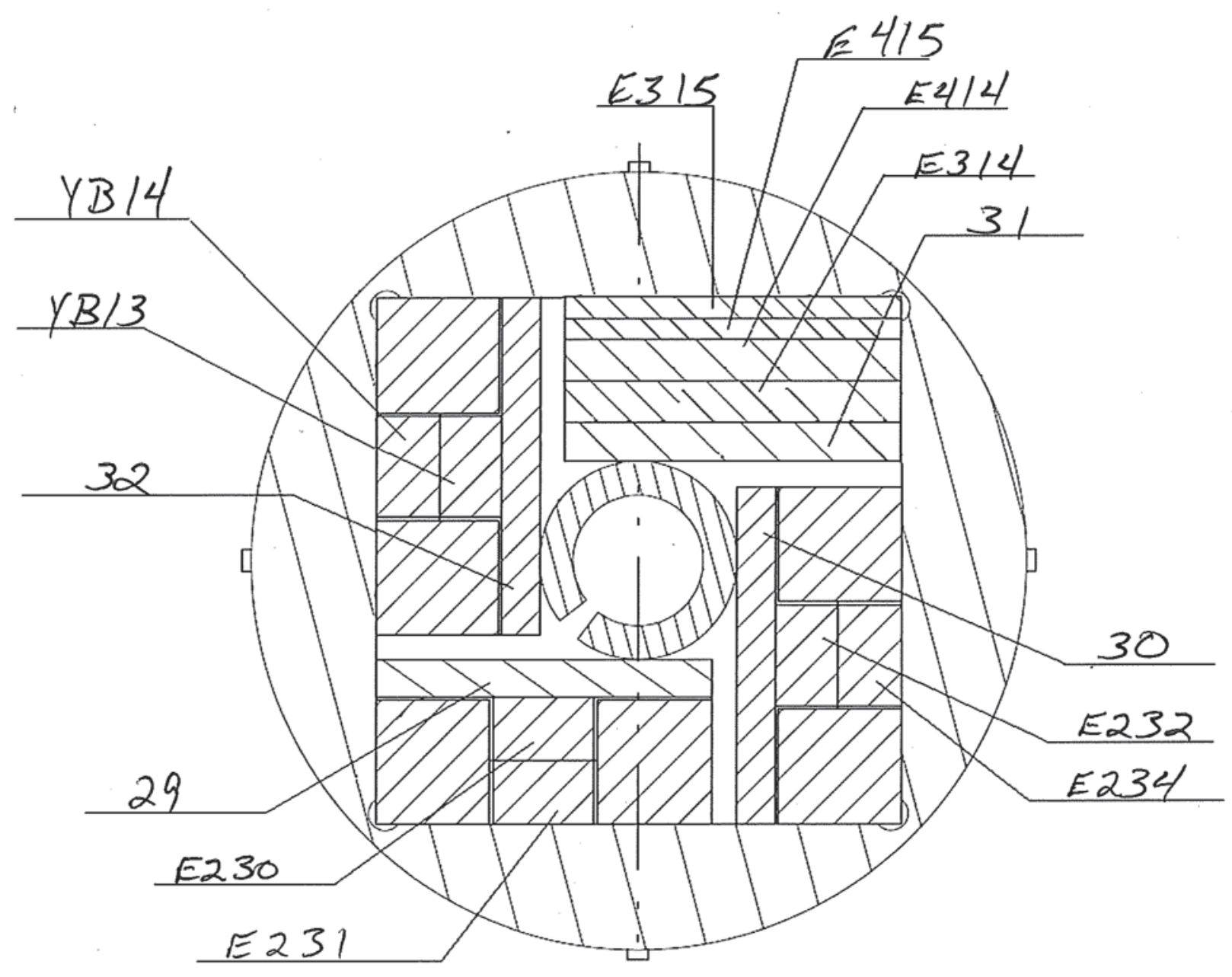


ES03

(17-08)

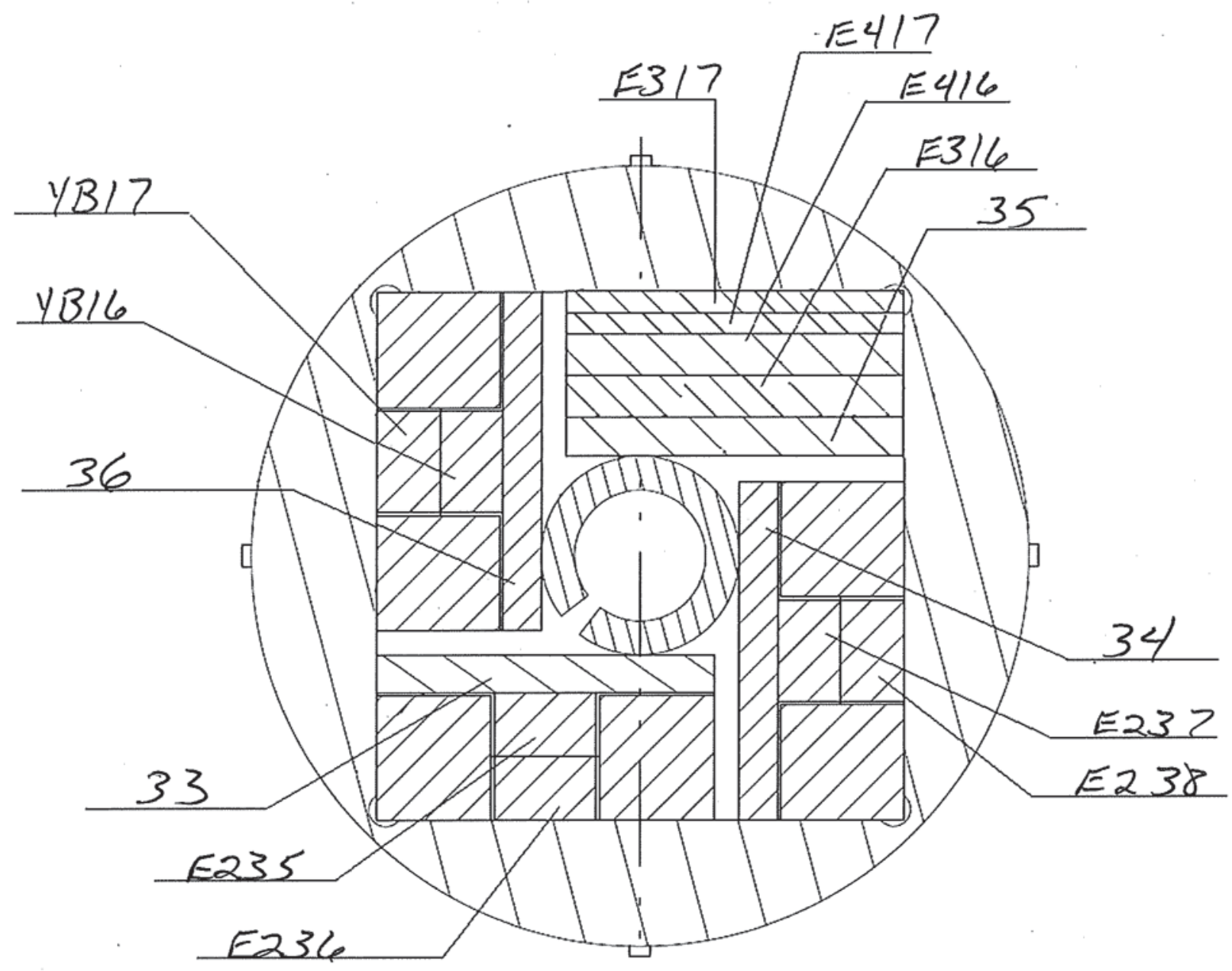


ES03

\begin{tabular}{|c|c|c|c|c|c|}
\hline $\begin{array}{r}\text { Design Diameter (mm) } \\
9.3\end{array}$ & $\begin{array}{r}\operatorname{Tavg}\left({ }^{\circ} \mathrm{C}\right) \\
268 \\
\text { Inner }\end{array}$ & $\begin{array}{r}\operatorname{Tavg}\left({ }^{\circ} \mathrm{C}\right) \\
254 \\
\text { Outer }\end{array}$ & & & Specimen Type \\
\hline \multirow{4}{*}{ Upper (X - Yc) } & E226 & E227 & 10 & 71 & SSJ3 (E) \\
\hline & E228 & E229 & 9 & 72 & SSJ3 (E) \\
\hline & E312 & E313 & $\mathrm{E} 412$ & E413 & MPC1 (E3) / MPC2 (E4) \\
\hline & B54-03 & YB12 & L7 & L8 & SSJ3 \\
\hline \multirow{4}{*}{$\operatorname{Mid}(X-Y c)$} & E230 & E231 & 7 & 74 & SSJ3 (E) \\
\hline & E232 & E234 & 24 & 75 & SSJ3 (E) \\
\hline & E314 & E315 & E414 & E415 & MPC1 (E3) / MPC2 (E4) \\
\hline & YB13 & YB14 & L9 & L10 & SSJ3 \\
\hline \multirow{4}{*}{ Lower $(X-Y c)$} & E235 & E236 & 6 & 77 & SSJ3 (E) \\
\hline & E237 & E238 & 31 & 78 & SSJ3 (E) \\
\hline & E316 & E317 & E416 & E417 & MPC1 (E3) / MPC2 (E4) \\
\hline & YB16 & YB17 & L11 & $\mathrm{L} 12$ & SSJ3 \\
\hline
\end{tabular}




\section{RABBIT ASSEMBLY: FS $\varnothing 3$ HOUSING}

\section{Eco?}

SN / IR NUMBER:

$18-\varnothing 7 / 2 \varnothing 839$

INNER

DIAMETER:

$\varnothing 9.52$

\section{HOLDER(S)}

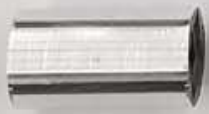

SN / IR NUMBER:

$17-\varnothing 6 / 2 \varnothing 787$

OUTER

DIAMETER:

$\varnothing 9.33$
SN / IR NUMBER:

$17-\not 87 / 20787$

OUTER

DIAMETER:

$\varnothing 9.32$

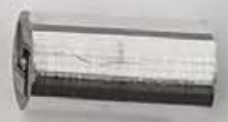

SN / IR NUMBER:

$17-\varnothing 8 / 28787$

OUTER

DIAMETER:

$\varnothing 9.33$ 
Capsule Number:

Irradiation Conditions

Irradiation Location

Design Temperature

First Cycle Goal

Irradiation Time

Irradiation Charge Number

Holder diameter

Fill Gas

Capsule Fabrication
ESO4

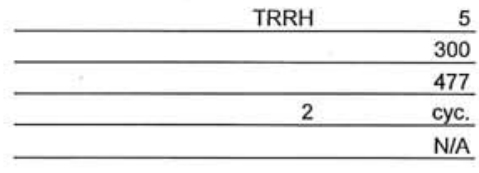

9.20

$\mathrm{mm}(0.3622 \mathrm{in})$ at $20^{\circ} \mathrm{C}$ Helium
Approvals

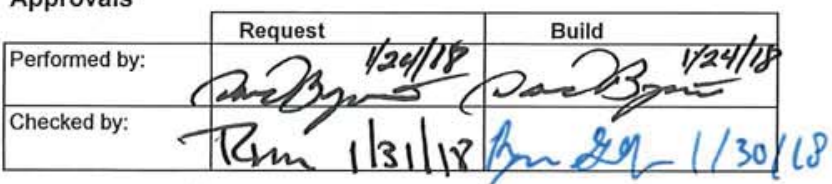

\begin{tabular}{|c|c|c|c|c|c|c|c|c|c|c|}
\hline & Drawing & Rev. & Part & Material & Count & Comment & MAT IR & FAB IR & ID & Mass (g) \\
\hline Housing & X3E020977A634 & A & 1 & Al 6061 & 1 & & 20838 & 20839 & $18-69$ & 4.2969 \\
\hline Housing end cap & X3E020977A634 & A & 2 & Al 4047 & 1 & & 20714 & 20714 & $17-28$ & 0.5157 \\
\hline Foil Spacer & S16-20-ESTEEL & 1 & 2 & Grafoil & 6 & & 19812 & 19812 & 6 Total & 0.0580 \\
\hline Wave Spring & S16-23-ESTEELB & 1 & CM08-L3-S17 & $17-7$ SS & 2 & & 20770 & 20770 & 2 Total & 0.2550 \\
\hline \multirow[t]{3}{*}{ Specimen Holder } & \multirow{3}{*}{ S16-22-ESTEEL } & \multirow[t]{3}{*}{1} & \multirow[t]{3}{*}{5} & \multirow[t]{3}{*}{ Al 6061} & \multirow[t]{3}{*}{3} & & \multirow[t]{3}{*}{20825} & \multirow[t]{3}{*}{20786} & $17-05$ & 1.2505 \\
\hline & & & & & & & & & $17-06$ & 1.2475 \\
\hline & & & & & & & & & $17-07$ & 1.2426 \\
\hline Spring Pin & S16-21-ESTEEL & 2 & 91610A207 & $18-8 \mathrm{SS}$ & 3 & & 20548 & 20548 & 3 Total & 0.4100 \\
\hline Chevron & S16-18-FUSSAM01 & 1 & 1 & EuroFer & 18 & 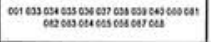 & 20777 & 20777 & 18 Total & 1.9850 \\
\hline \multirow[t]{10}{*}{ Thermometry } & \multirow[t]{11}{*}{ S16-18-FUSSAM01 } & \multirow[t]{11}{*}{1} & \multirow[t]{11}{*}{7} & \multirow[t]{11}{*}{$\mathrm{SiC}$} & \multirow[t]{11}{*}{12} & \multirow[b]{11}{*}{ ' } & \multirow[t]{11}{*}{19759} & \multirow[t]{11}{*}{20840} & 37 & 0.0992 \\
\hline & & & & & & & & & 38 & 0.0995 \\
\hline & & & & & & & & & 39 & 0.1005 \\
\hline & & & & & & & & & 40 & 0.1000 \\
\hline & & & & & & & & & 41 & 0.1001 \\
\hline & & & & & & & & & 42 & 0.0979 \\
\hline & & & & & & & & & 43 & 0.1003 \\
\hline & & & & & & & & & 44 & 0.0997 \\
\hline & & & & & & & & & 45 & 0.0991 \\
\hline & & & & & & & & & \begin{tabular}{l|}
46 \\
47
\end{tabular} & 0.0993 \\
\hline \multirow[t]{15}{*}{ SSJ3 } & & & & & & & & & 48 & $\begin{array}{l}0.0994 \\
0.1001 \\
\end{array}$ \\
\hline & S16-18-FUSSAM01 & 1 & 2 & EuroFer & 20 & & 20778 & 20778 & E239 & 0.2607 \\
\hline & & & & & & & $\begin{array}{l}20778 \\
20778\end{array}$ & 20778 & & 0.2599 \\
\hline & & & & & & & 20778 & 20778 & & $\begin{array}{l}0.2617 \\
0.2601 \\
\end{array}$ \\
\hline & & & & & & & 20778 & 20778 & E243 & 0.2592 \\
\hline & & & & & & & 20778 & 20778 & E245 & 0.2585 \\
\hline & & & & & & & 20778 & 20778 & E247 & 0.2608 \\
\hline & & & & & & & 20778 & 20778 & $\begin{array}{l}\text { E248 } \\
E 249\end{array}$ & $\begin{array}{l}0.2597 \\
0.2579\end{array}$ \\
\hline & & & & & & & 20778 & 20778 & E250 & 0.2626 \\
\hline & & & & & & & 20778 & 20778 & E251 & 0.2601 \\
\hline & & & & & & & $207 / 8$ & $207 / 8$ & E252 & 0.2594 \\
\hline & & & & & & & $\frac{20841}{20841}$ & $\frac{20841}{20841}$ & YB19 & 0.2679 \\
\hline & & & & & & & 20841 & 20841 & YB20 & $\begin{array}{l}0.2693 \\
0.2686\end{array}$ \\
\hline & & & & & & & 20841 & 20841 & YB22 & 0.2678 \\
\hline & & & & & & & 20841 & 20841 & YB23 & 0.2657 \\
\hline MPC1 & S16-18-FUSSAM01 & 1 & 5 & EuroFer & 12 & & 20841 & 20841 & B54-09 & 0.2573 \\
\hline & & & & & 12 & & 20780 & 20780 & $\begin{array}{l}\text { E318 } \\
\text { E319 }\end{array}$ & 0.1090 \\
\hline & & & & & & & & & E320 & 0.1050 \\
\hline & & & & & & & & & E321 & 0.1090 \\
\hline & & & & & & & & & E322 & 0.1120 \\
\hline MPC2 & S16-18-FUSSAM01 & 1 & 6 & For & 2 & & & & E323 & 0.1080 \\
\hline & & & & Latur i & 12 & & 20101 & 20181 & $\begin{array}{l}\text { E418 } \\
\text { E419 }\end{array}$ & 0.2480 \\
\hline & & & & & & & & & E420 & 0.2550 \\
\hline & & & & & & & & & E421 & 0.2580 \\
\hline & & & & & & & & & E422 & 0.2540 \\
\hline & & & & & & & & & \begin{tabular}{r|} 
E423 \\
Total Mass
\end{tabular} & $\begin{array}{r}0.2340 \\
19.3286\end{array}$ \\
\hline & & & & & & & & & Specimen Mass & 6.8723 \\
\hline Assembly & & & & & & & & & Internal Mass & 14.5160 \\
\hline
\end{tabular}

Assembly Drawing S16-20-ESTEE

Rev.

X3E020977A633

Fill Gas Helium 
Capsule Number:

\section{Irradiation Conditions}

Irradiation Location

Design Temperature

First Cycle Goal

Irradiation Time

Irradiation Charge Number

Holder diameter

Fill Gas

Capsule Fabrication

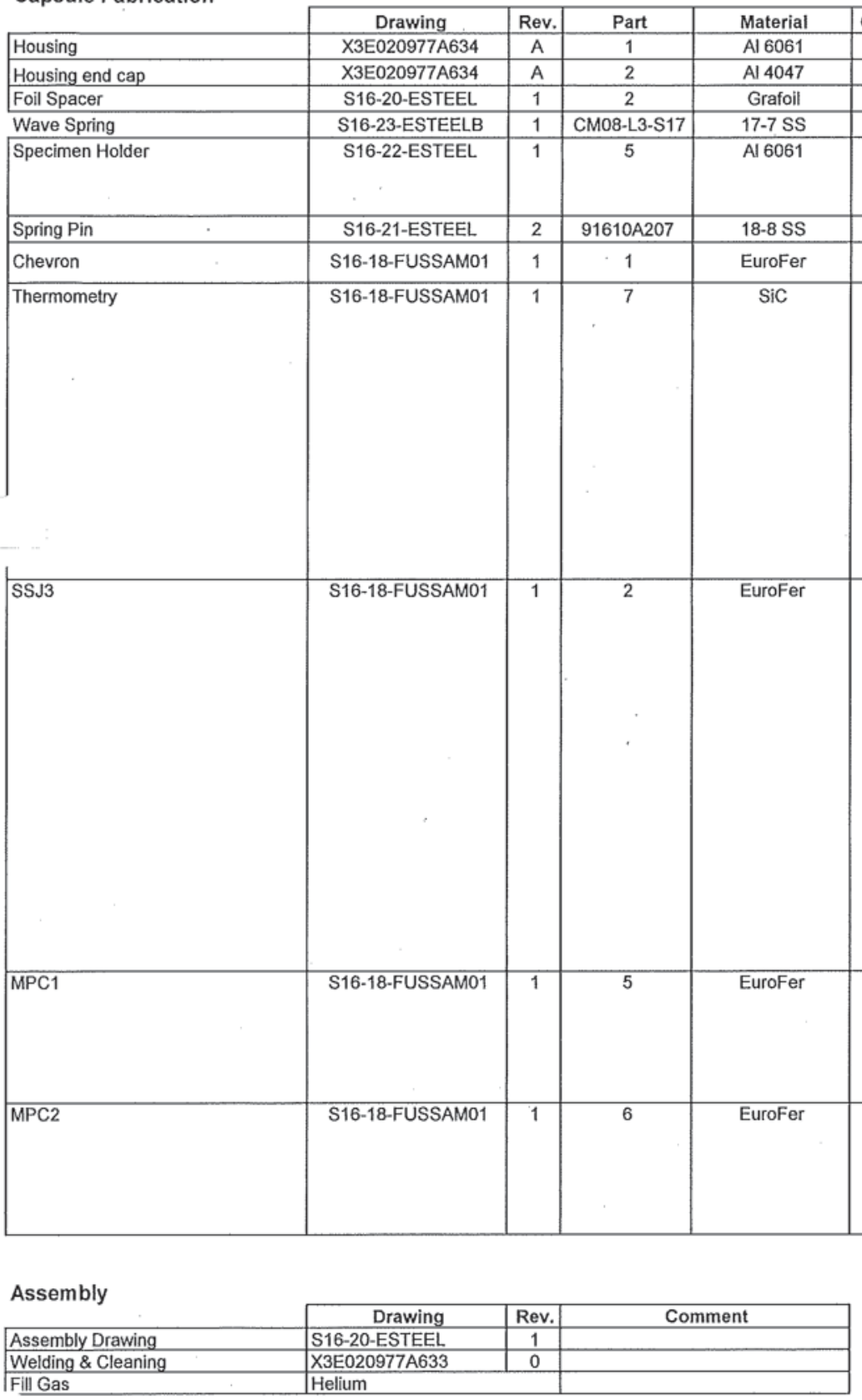

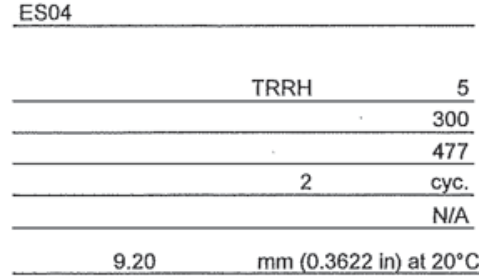
Helium

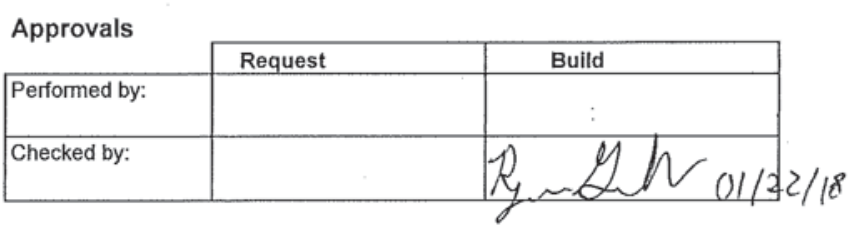

\section{Count Comment

ount

\begin{tabular}{|l|l|l}
\hline 1 & & 208 \\
\hline 1 & & 207 \\
\hline 6 & & 198 \\
\hline 2 & & 2077 \\
\hline
\end{tabular}

\begin{tabular}{l|l|l}
\hline 3 & 208 \\
& &
\end{tabular}

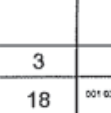

\begin{tabular}{|c|c|}
\hline 18 & 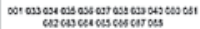 \\
\hline 12 & \\
\hline
\end{tabular}

19759

\begin{tabular}{l|l}
\hline 19759 & 20777 \\
\hline
\end{tabular}

\begin{tabular}{ll}
20 \\
\hline 20
\end{tabular}

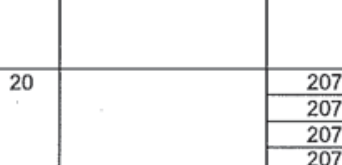

\begin{tabular}{|c|c|}
\hline MAT IR & F \\
\hline 20838 & 2089 \\
\hline 20714 & 2071 \\
\hline 19812 & 1 \\
\hline 20770 & 2077 \\
\hline 20825 & 2078 \\
\hline
\end{tabular}

\begin{tabular}{c|c} 
FAB IR & ID \\
\hline 20839 & \\
\hline
\end{tabular}

\begin{tabular}{|r|r|r|} 
& $18-69$ & \\
\hline 20714 & $17-28$ & 0.5157 \\
\hline
\end{tabular}

\begin{tabular}{l|r|r|}
\hline 19812 & 6 Total & 0.0580 \\
\hline
\end{tabular}

\begin{tabular}{|r|r|r|}
\hline 2812 & 6 Total & 0.0580 \\
\hline \multirow{3}{*}{0770} & 2 Total & 0.2550 \\
\cline { 2 - 3 } & $17-05$ & 1.2505 \\
\cline { 2 - 3 } & $17-06$ & 1.2475 \\
\cline { 2 - 3 } & $17-07$ & 1.2426 \\
& 370 & 0.4100 \\
\hline
\end{tabular}


ES04

(17-05)

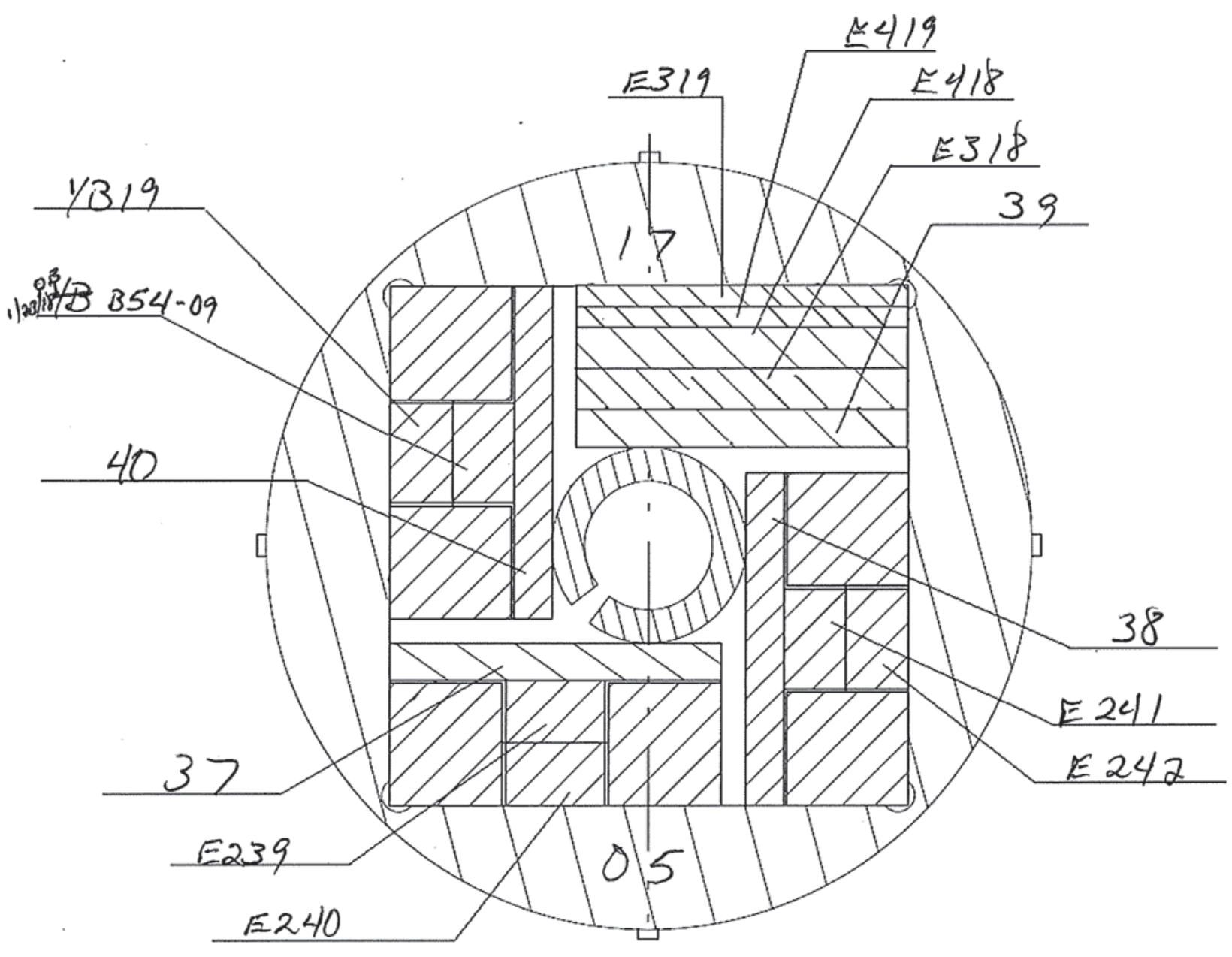


ES04

(17-06)

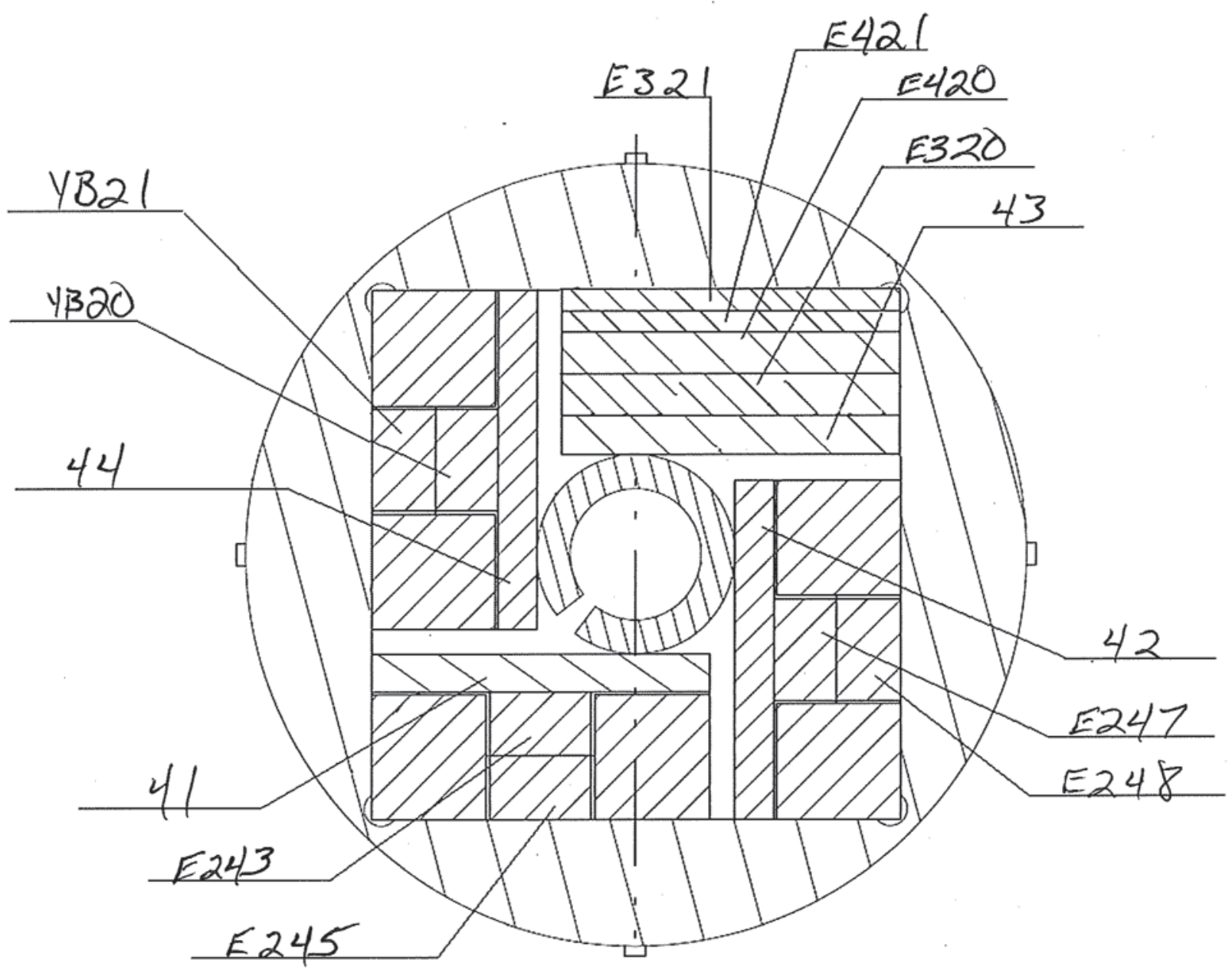


ES04

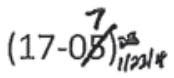

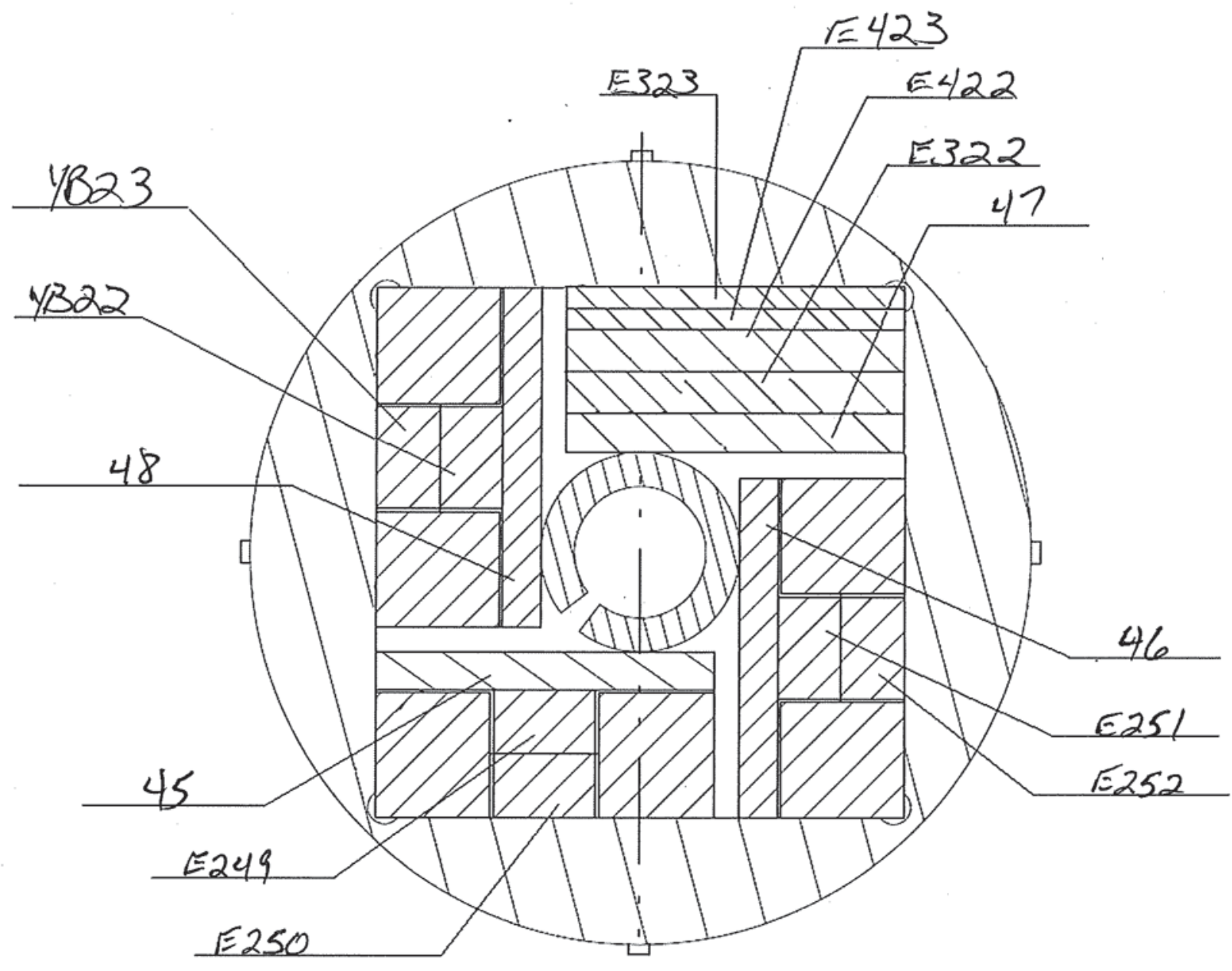




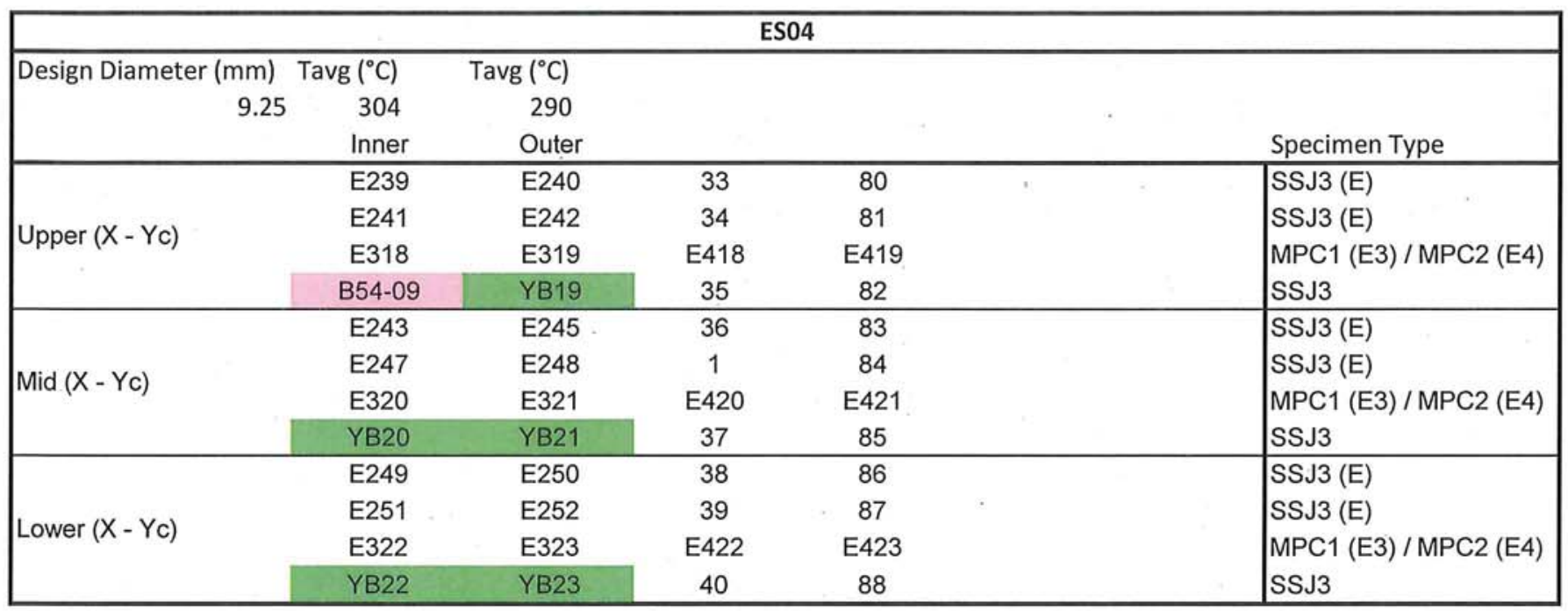




\section{RABBIT ASSEMBLY: $E S \not \varnothing 4$ HOUSING}

\section{ESOM}

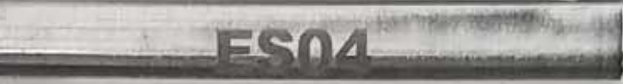

SN / IR NUMBER:

$18-\underline{6} 9 / 20883 \underline{9}$

INNER

DIAMETER:

$\varnothing 9.53$

HOLDER(S)

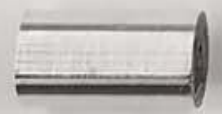

SN / IR NUMBER:

$17-\varnothing 5 / 2 \varnothing 786$

$17-\not 87 / 20786$

OUTER

DIAMETER:

OUTER

DIAMETER:

$\varnothing 9.3 \varnothing$
SN / IR NUMBER:

SN / IR NUMBER:

$17-\not 6 / 20786$

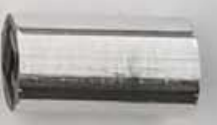

OUTER

DIAMETER:

$\phi 9.2 \underline{9}$ $\varnothing 9.28$ 
Capsule Number:

Irradiation Conditions

Irradiation Location

Design Temperature

First Cycle Goal

Irradiation Time

Irradiation Charge Number

Holder diameter

Fill Gas

Capsule Fabrication
ES05

\begin{tabular}{rr} 
TRRH & 5 \\
\hline & 325 \\
\hline 2 & 477 \\
\hline cyc. \\
\hline N/A \\
\hline
\end{tabular}

9.20

$\mathrm{mm}(0.3622 \mathrm{in})$ at $20^{\circ} \mathrm{C}$ Helium

\section{Approvals}

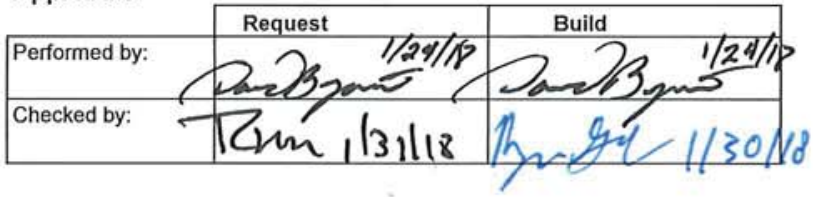

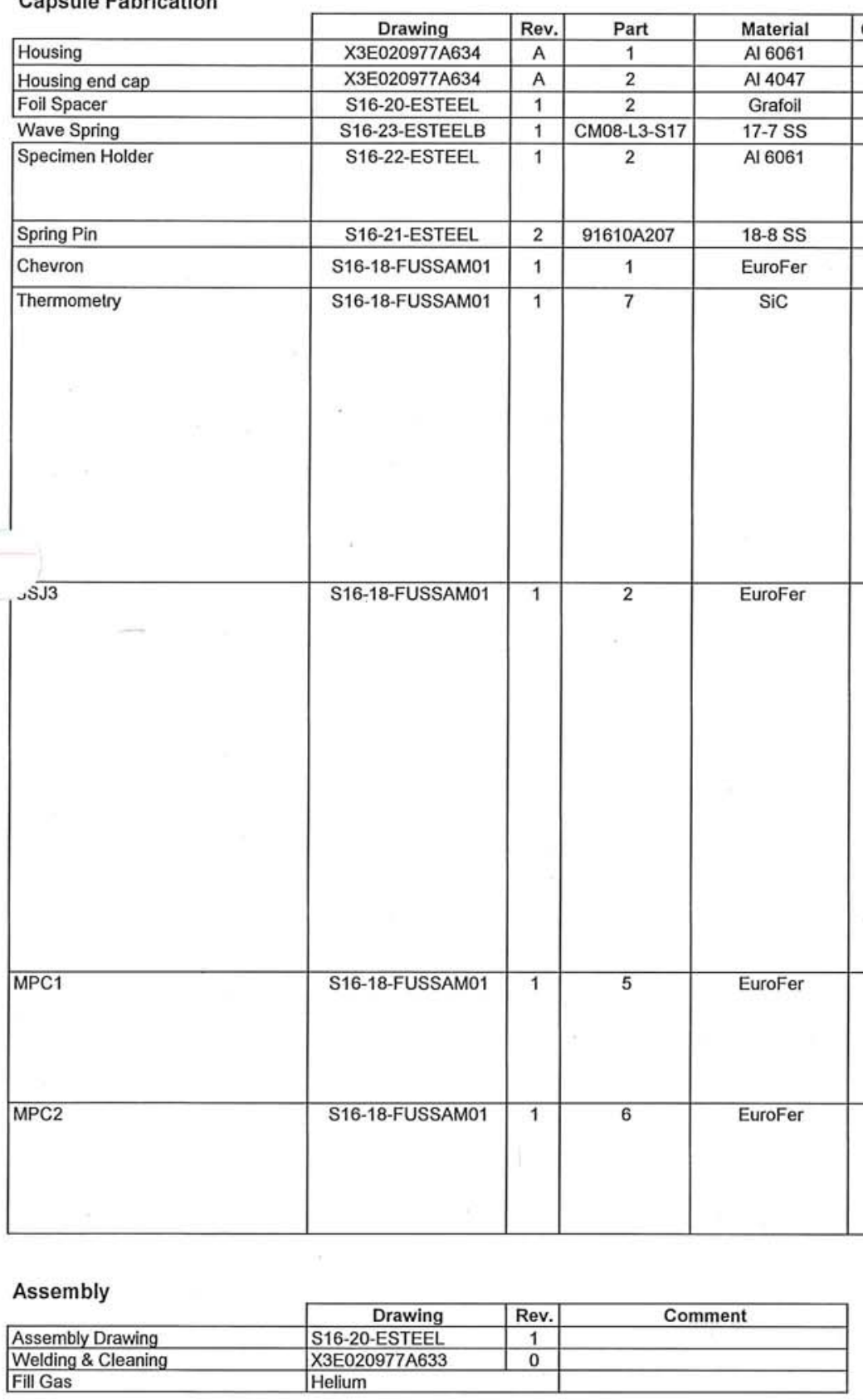

\begin{tabular}{|l|l|} 
Count & Comment \\
\hline
\end{tabular}

\begin{tabular}{|c|c|c|}
\hline 1 & Comment & MAT IR \\
\hline
\end{tabular}

\begin{tabular}{|l|l|r}
\hline 1 & & 20838 \\
\hline 1 & & 20714 \\
\hline
\end{tabular}

\begin{tabular}{l|l|l}
1 & & 20714 \\
\hline 6 & & 19812
\end{tabular}

\begin{tabular}{|l|l|l|r|r|r|}
\hline 2 & & 19812 & 19812 & 6 Total & 0.0560 \\
\hline 3 & & 20770 & 20770 & 2 Total & 0.2570 \\
\hline
\end{tabular}

\begin{tabular}{|l|l|l|r|r|}
\hline 3 & 20825 & \multirow{2}{*}{20783} & $17-07$ & 1.2331 \\
\cline { 4 - 5 } & & & $17-08$ & 1.2275 \\
\cline { 4 - 5 } & & & $17-10$ & 1.2262 \\
\hline
\end{tabular}

\begin{tabular}{|c|c|c|c|c|c|}
\hline 3 & & 20548 & 20548 & 3 Total & 0.4120 \\
\hline 18 & 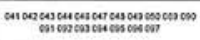 & 20777 & 20777 & 18 Total & 1.9770 \\
\hline \multirow[t]{2}{*}{12} & & \multirow[t]{2}{*}{19759} & \multirow[t]{2}{*}{20840} & 85 & 0.0996 \\
\hline & & & & 50 & 0.0978 \\
\hline
\end{tabular}


ES05

(17-07)

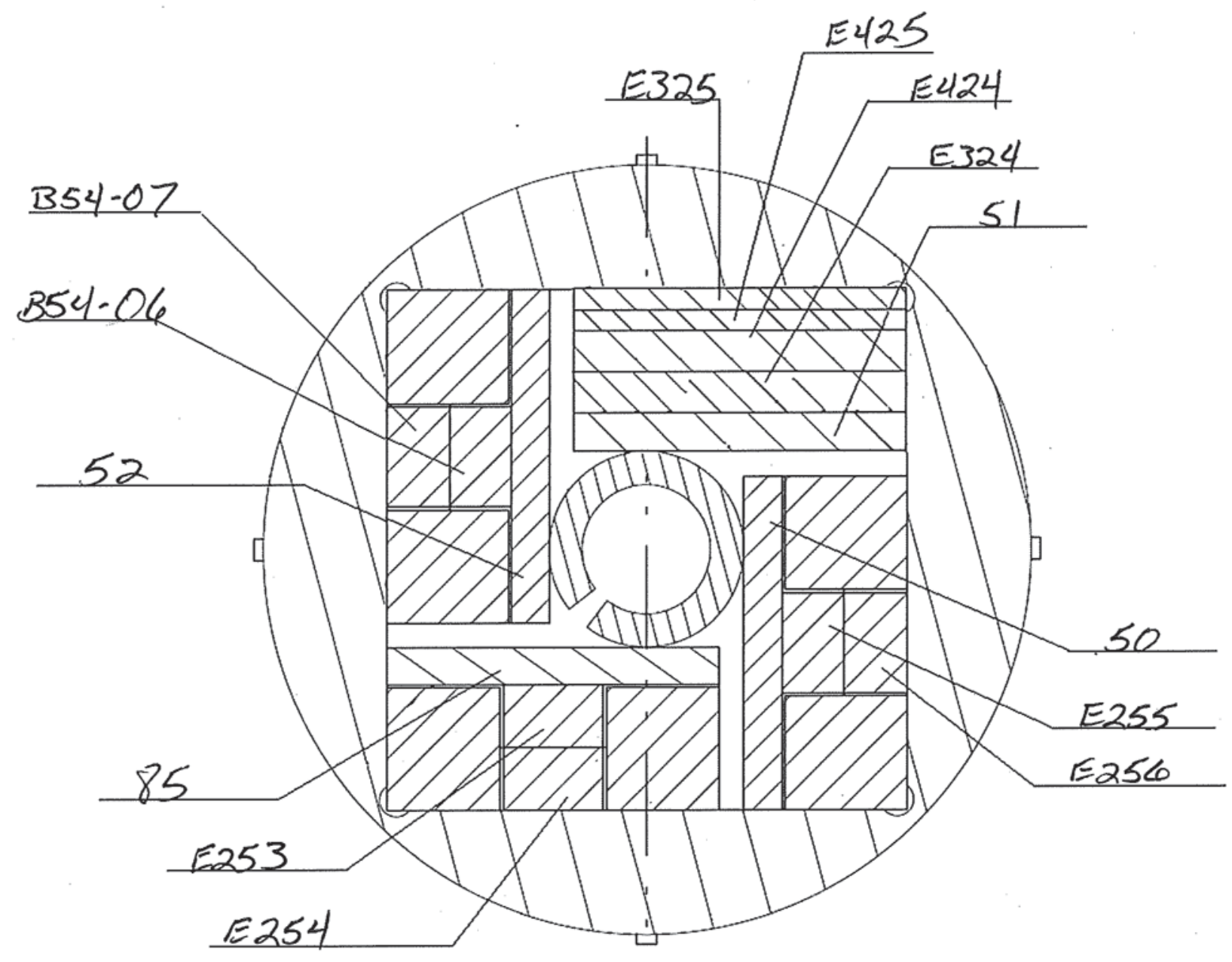


ES05

(17-08)

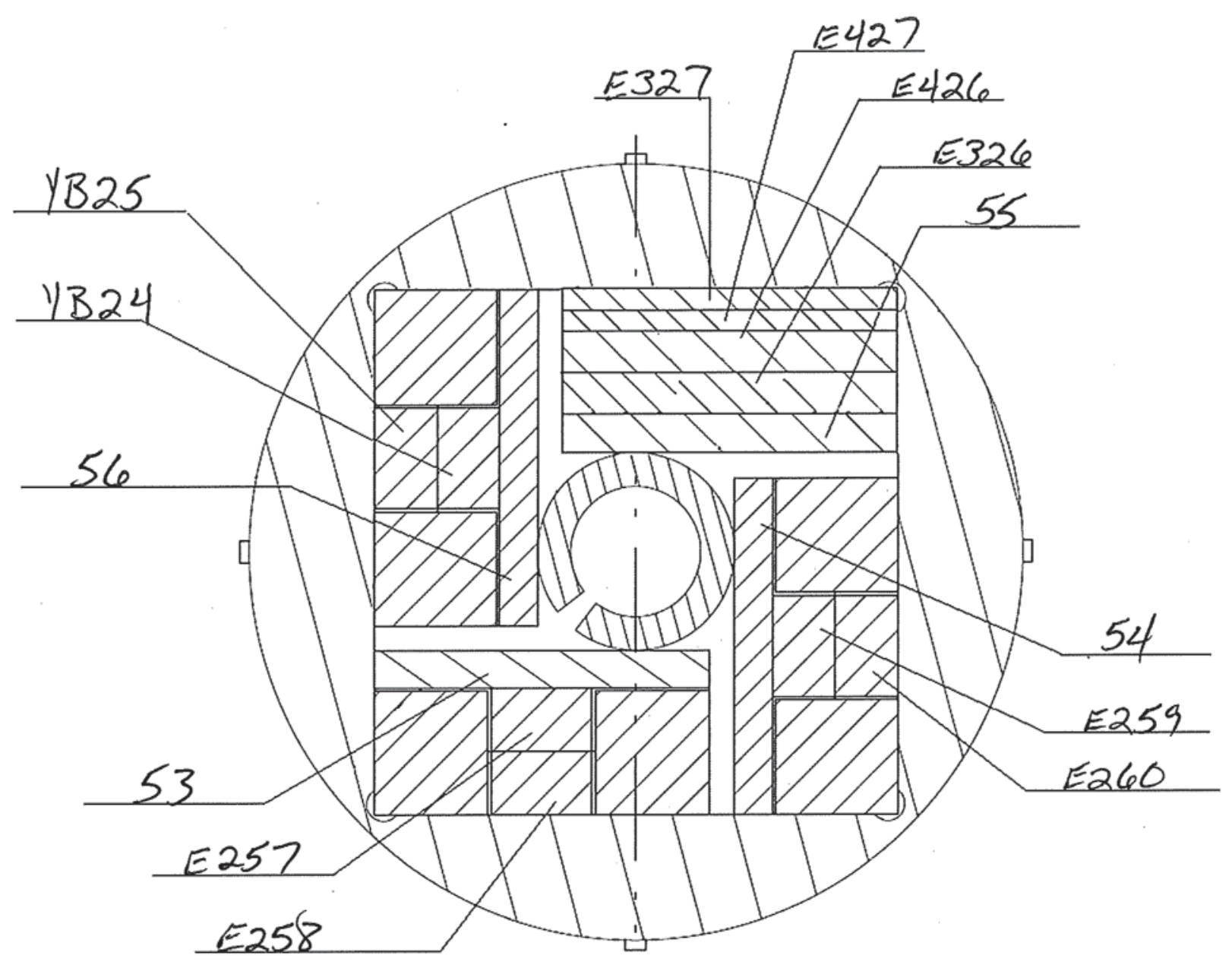


ES05

(17-10)

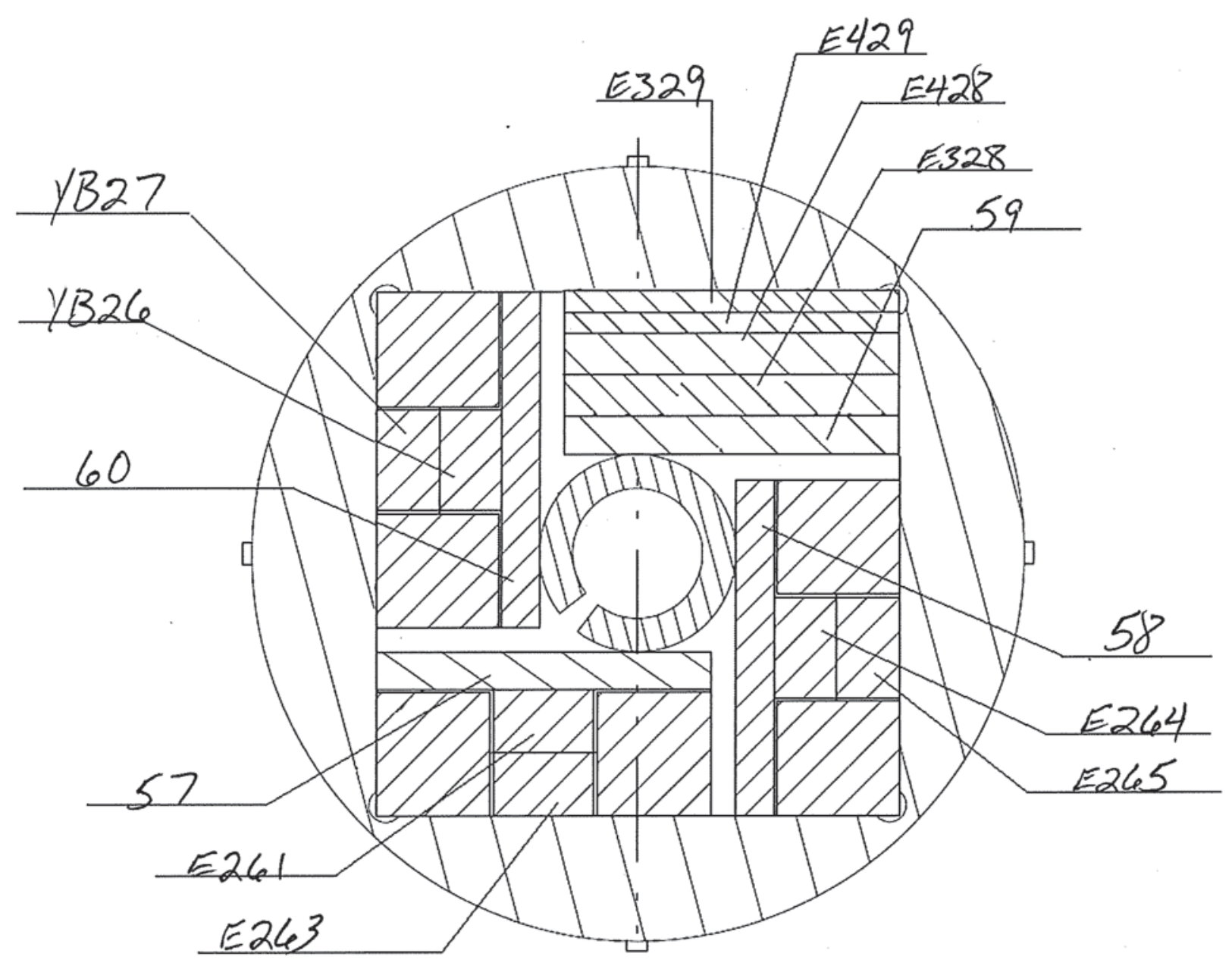


ES05

\begin{tabular}{|c|c|c|c|c|c|}
\hline \multirow{3}{*}{$\begin{array}{r}\text { Design Diameter ( } \mathrm{mm}) \\
9.2\end{array}$} & $\operatorname{Tavg}\left({ }^{\circ} \mathrm{C}\right)$ & $\operatorname{Tavg}\left({ }^{\circ} \mathrm{C}\right)$ & & & \multirow[b]{3}{*}{ Specimen Type } \\
\hline & 330 & 316 & & & \\
\hline & Inner & Outer & & & \\
\hline \multirow{4}{*}{ Upper $(X-Y c)$} & E253 & E254 & 41 & 89 & SSJ3 (E) \\
\hline & E255 & E256 & 42 & 90 & SSJ3 (E) \\
\hline & E324 & E325 & E424 & E425 & MPC1 (E3) / MPC2 (E4) \\
\hline & B54-06 & B54-07 & 43 & 91 & SSJ3 \\
\hline \multirow{4}{*}{$\operatorname{Mid}(X-Y c)$} & E257 & E258 & 44 & 92 & SSJ3 (E) \\
\hline & E259 & E260 & 46 & 93 & SSJ3 (E) \\
\hline & E326 & E327 & E426 & E427 & MPC1 (E3) / MPC2 (E4) \\
\hline & YB24 & YB25 & 47 & 94 & SSJ3 \\
\hline \multirow{4}{*}{ Lower $(X-Y c)$} & E261 & E263 & 48 & 95 & SSJ3 (E) \\
\hline & E264 & E265 & 49 & 96 & SSJ3 (E) \\
\hline & E328 & E329 & E428 & E429 & MPC1 (E3) / MPC2 (E4) \\
\hline & YB26 & YB27 & 50 & 97 & SSJ3 \\
\hline
\end{tabular}




\section{RABBIT ASSEMBLY: $E S \varnothing 5$ HOUSING}

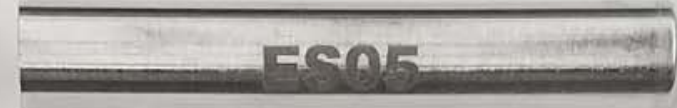

SN / IR NUMBER:

$18-\underline{6} 4 / 20839$

INNER

DIAMETER:

$\varnothing 9.52$

\section{HOLDER(S)}
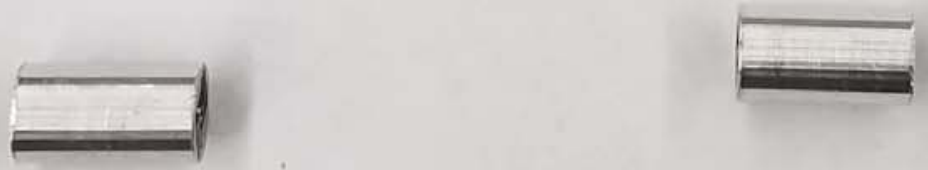

SN / IR NUMBER:

SN / IR NUMBER:

$17-\varnothing 7 / 2 \varnothing 783$

OUTER

DIAMETER:

$\varnothing 9.25$
OUTER

DIAMETER:

$\varnothing \underline{q} .24$

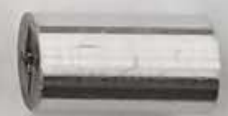

SN / IR NUMBER:

$17-10 / 28783$

OUTER DIAMETER:

$\varnothing 9.25$ 
Capsule Number:

\section{Irradiation Conditions}

Irradiation Location

Design Temperature

First Cycle Goal

Irradiation Time

Irradiation Charge Number

Holder diameter

Fill Gas

Capsule Fabrication
ES06

\begin{tabular}{rrr} 
& TRRH & 5 \\
\hline & 350 \\
\hline & 477 \\
\hline 2 & cyc. \\
\hline & N/A \\
\hline 9.20 & mm (0.3622 in) at $20^{\circ} \mathrm{C}$ \\
Helium \\
\hline
\end{tabular}

\section{Approvals}

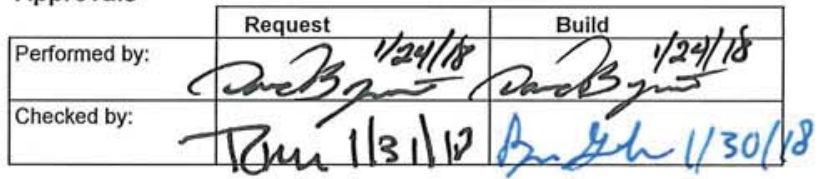

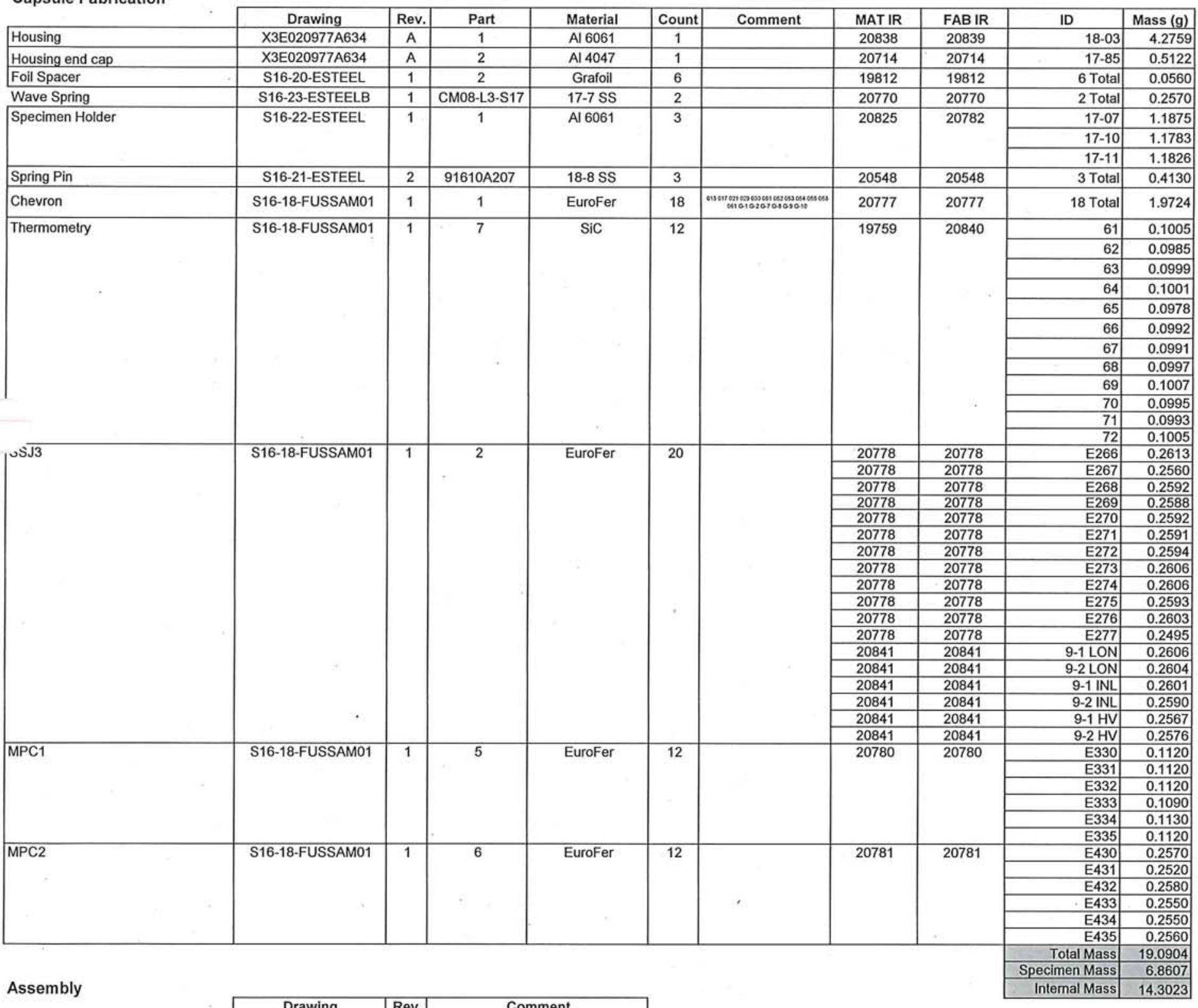

\begin{tabular}{|l|l|c|c|}
\cline { 2 - 4 } \multicolumn{1}{c|}{} & \multicolumn{1}{c|}{ Drawing } & Rev. & \multicolumn{1}{c|}{ Comment } \\
\hline Assembly Drawing & S16-20-ESTEEL & 1 & \\
\hline Welding \& Cleaning & X3E020977A633 & 0 & \\
\hline Fill Gas & Helium & & \\
\hline
\end{tabular}


Capsule Number:

Irradiation Conditions

Irradiation Location

Design Temperature

First Cycle Goal

Irradiation Time

Irradiation Charge Number

Holder diameter

Fill Gas

Capsule Fabrication

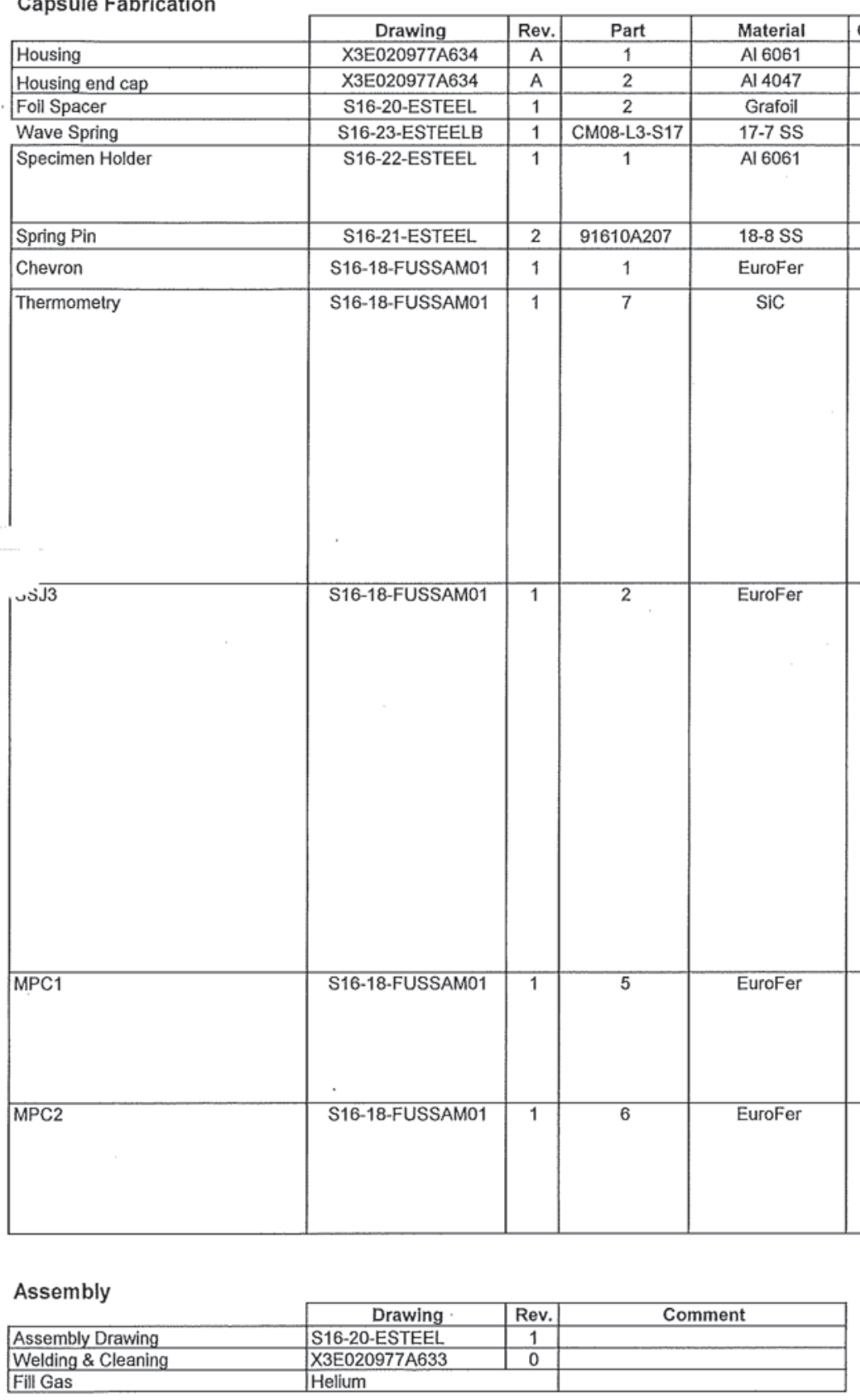

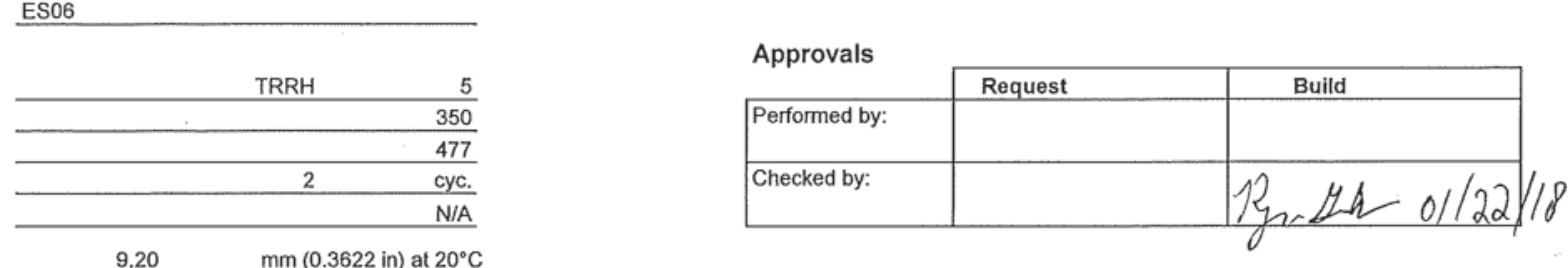

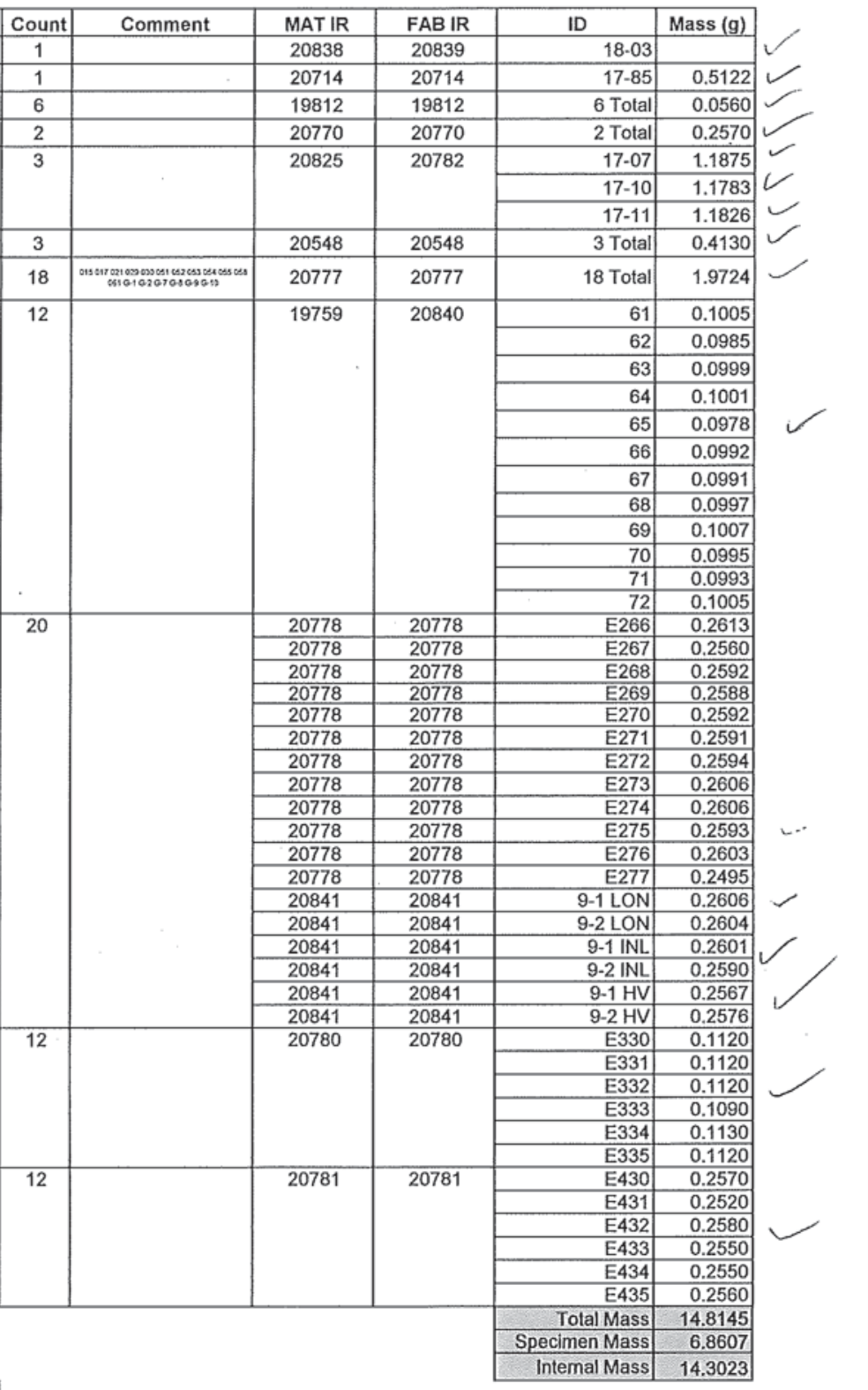


ES06

(17-07)

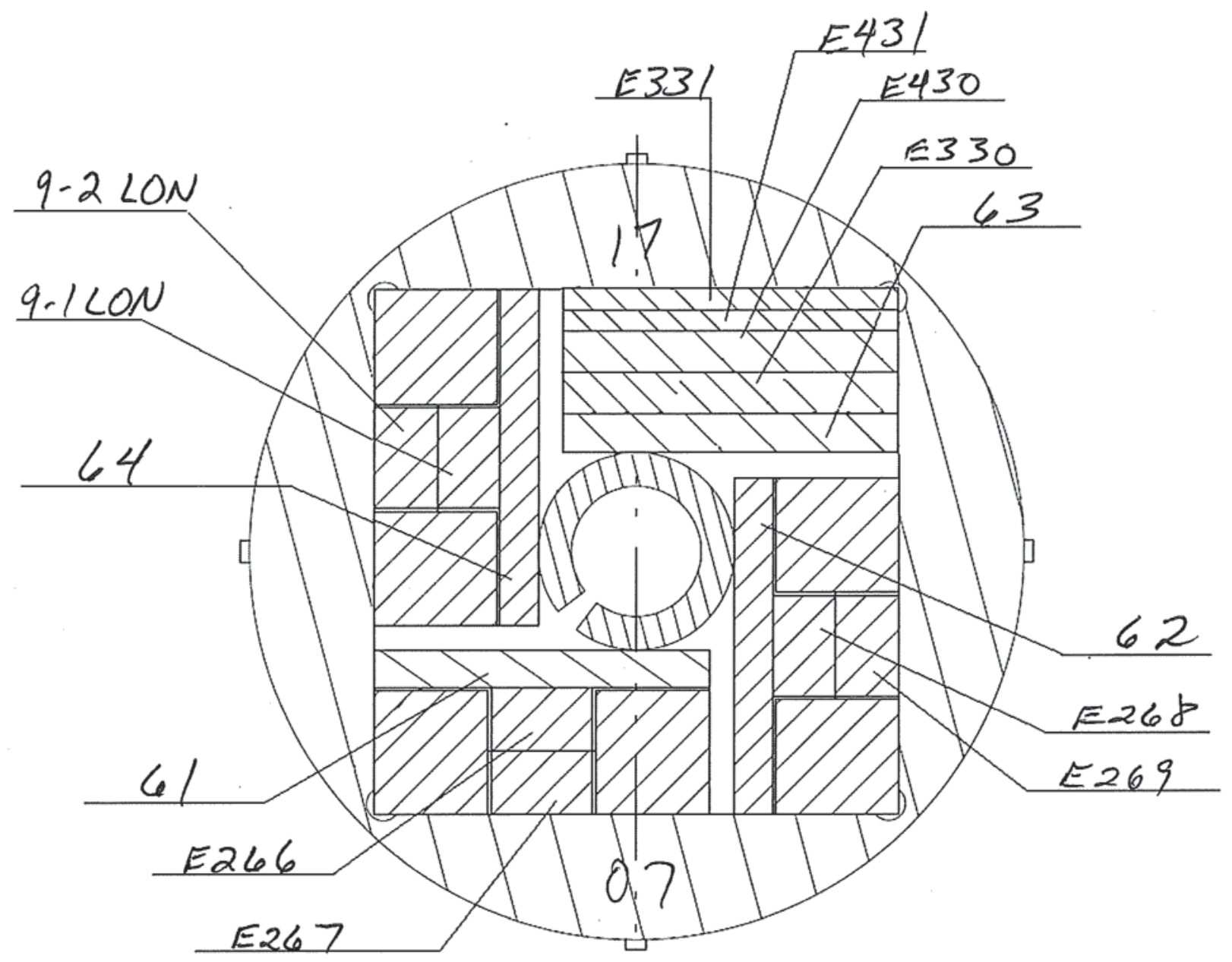




\section{ES06}

(17-10)

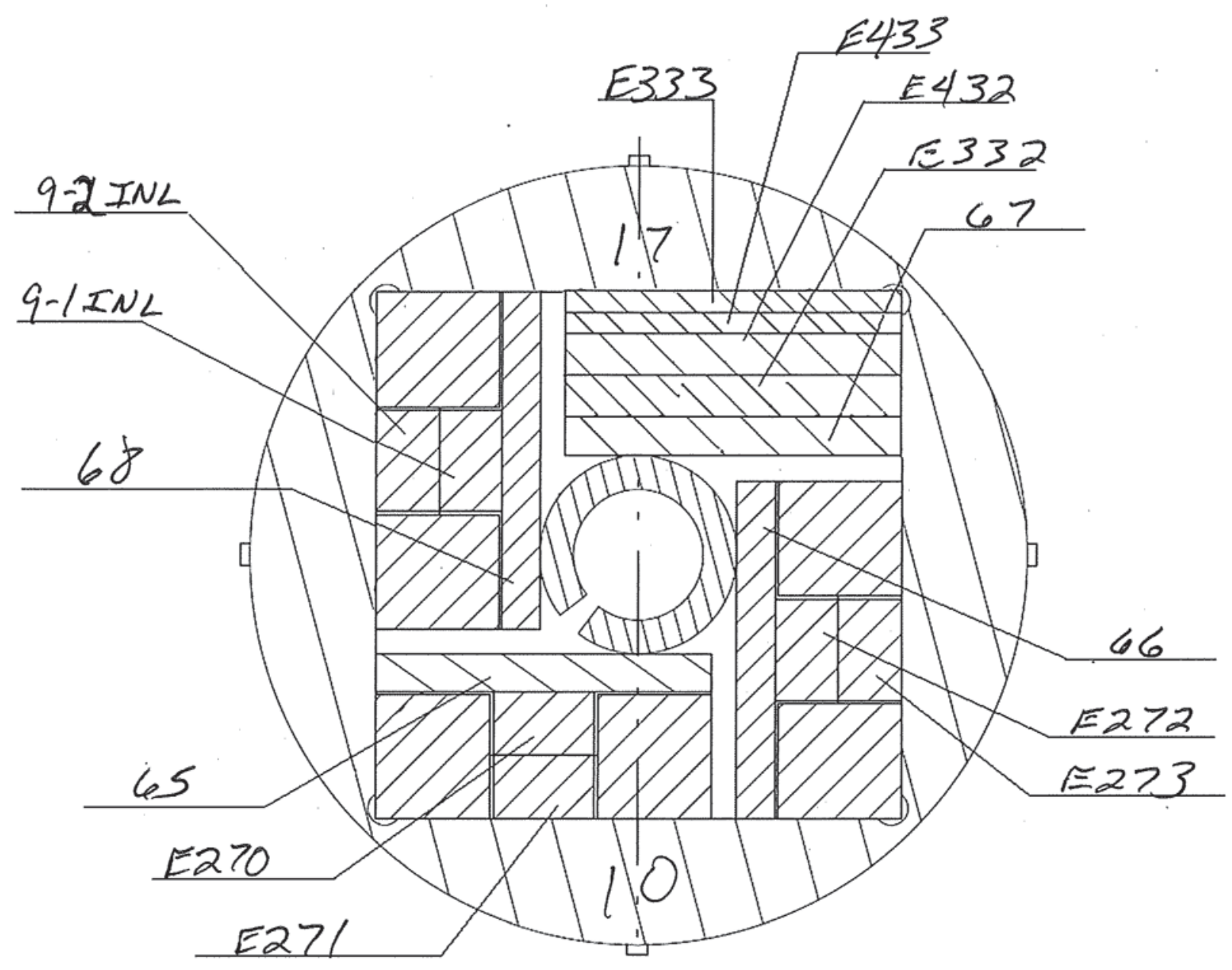


ES06

(17-11)

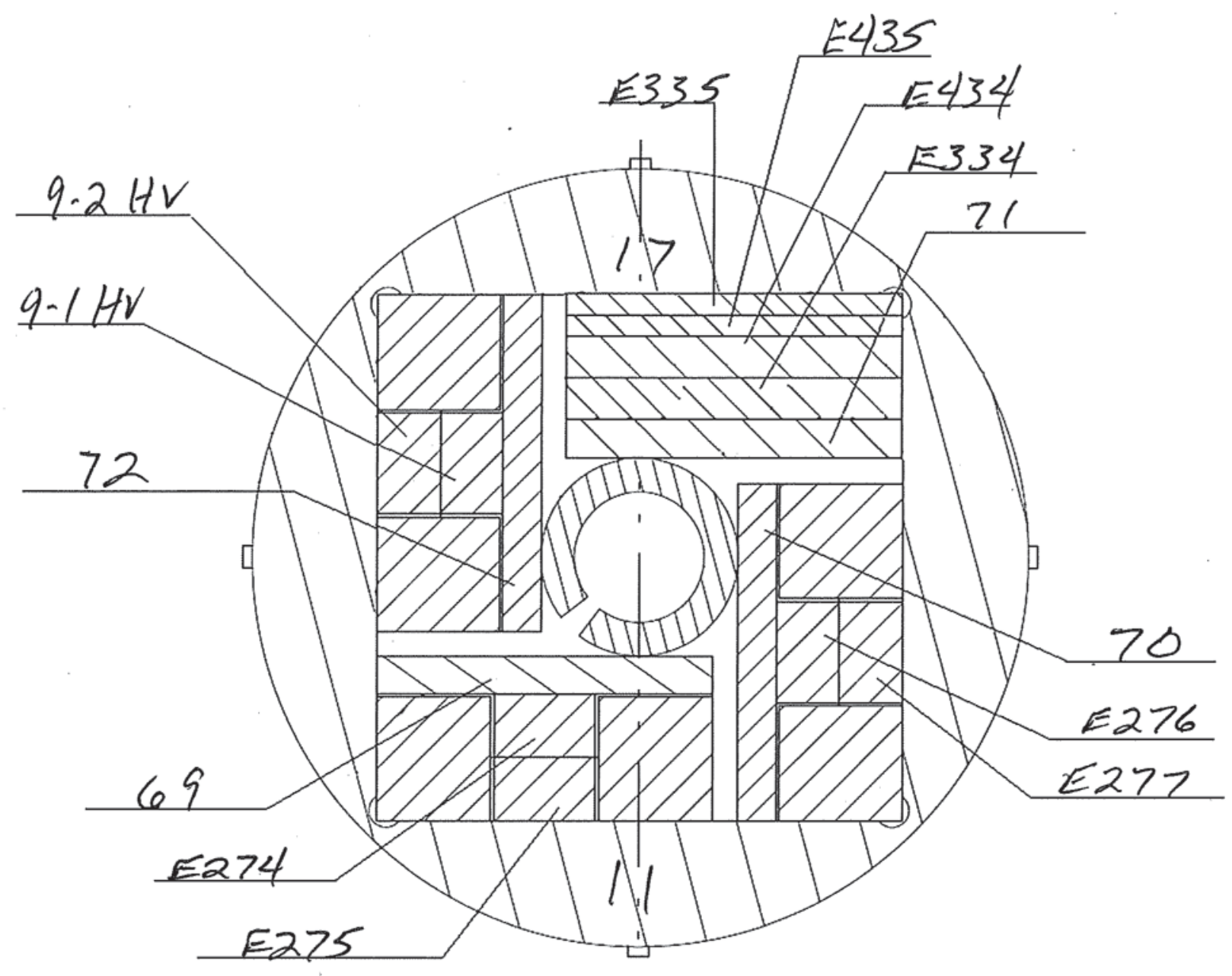


ES06

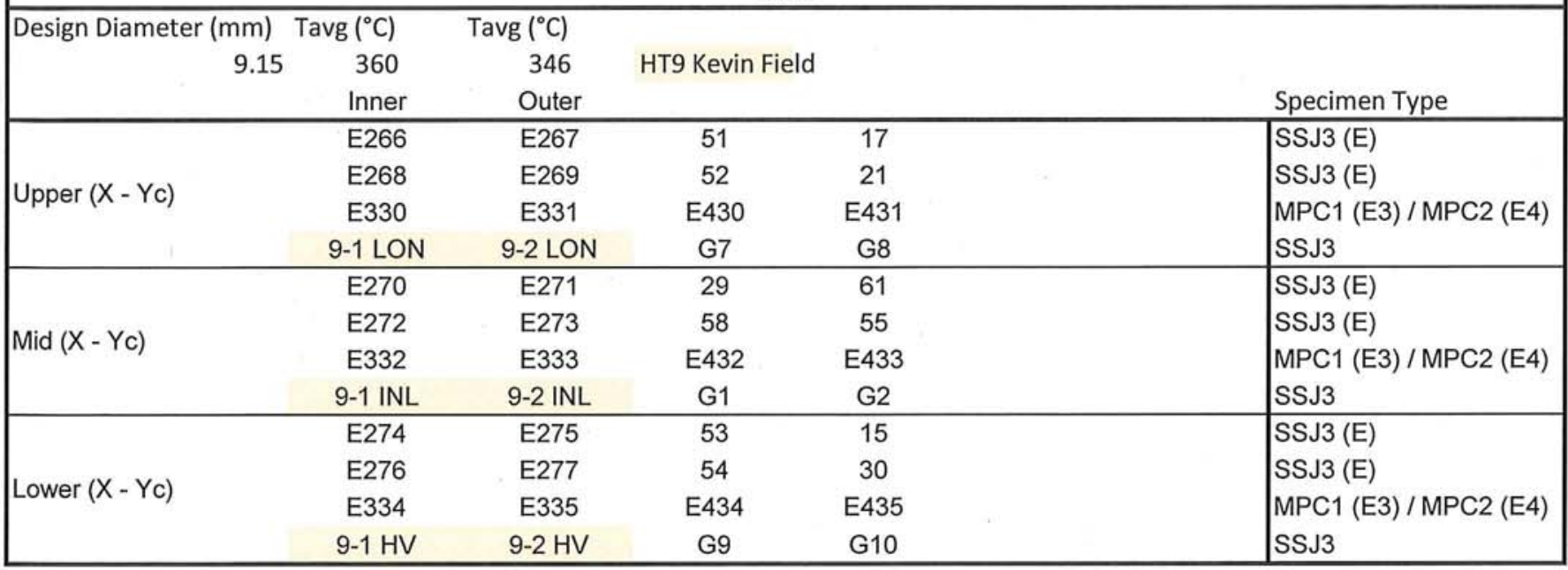




\section{RABBIT ASSEMBLY: $E S \phi \underline{6}$ HOUSING}

\section{ESO6}

SN / IR NUMBER:

$18-\not 03 / 2 \varnothing 839$

INNER

DIAMETER:

$\varnothing 9.52$

\section{$\operatorname{HOLDER}(\mathrm{S})$}

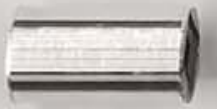

SN / IR NUMBER:

SN / IR NUMBER:

SN / IR NUMBER:

$17-\varnothing 7 / 20782$

$17-10 / 2 \varnothing 782$

$17-11 / 20782$

OUTER

DIAMETER:

$\phi \underline{q} .18$
OUTER

DIAMETER:

$\phi \underline{9} .19$
OUTER DIAMETER:

$\varnothing 9.2 \varnothing$ 
Capsule Number:

Irradiation Conditions

Irradiation Location

Design Temperature

First Cycle Goal

Irradiation Time

Irradiation Charge Number

Holder diameter

Fill Gas

Capsule Fabrication

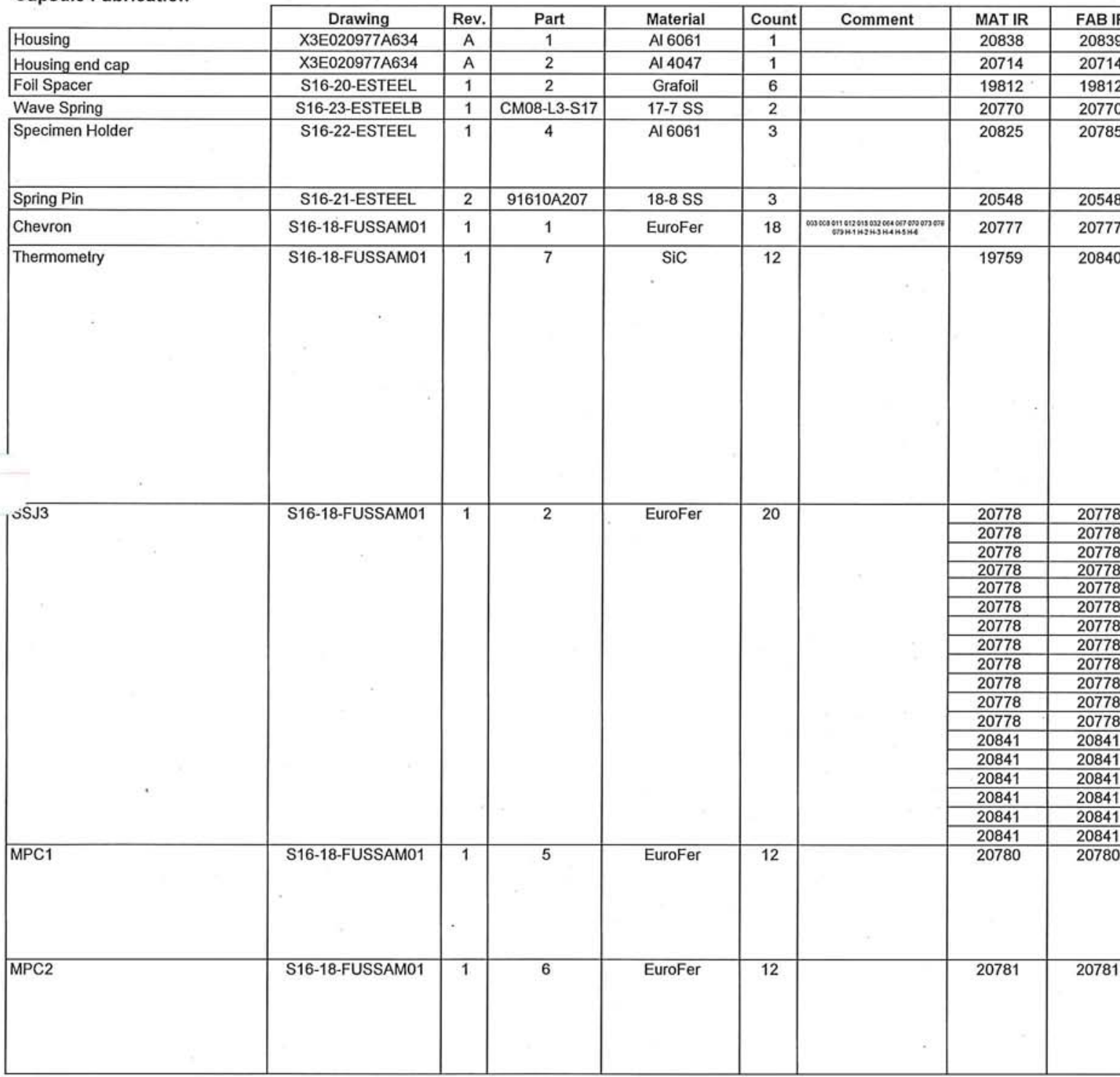

\section{Assembly}

Assembly Drawing

Welding \& Cleaning

Fill Gas
ES07

\begin{tabular}{rr} 
TRRH & 5 \\
\hline & 375 \\
\hline & 477 \\
\hline 2 & cyc. \\
\hline N/A \\
\hline
\end{tabular}

9.20

$\mathrm{mm}(0.3622 \mathrm{in})$ at $20^{\circ} \mathrm{C}$

Helium

\section{Approvals}

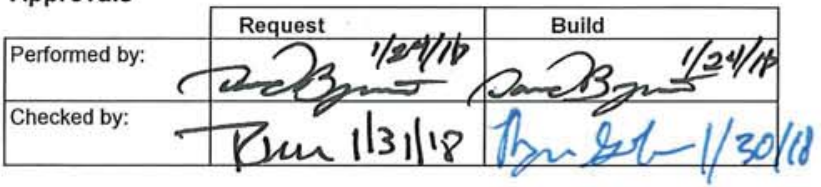


Capsule Number:

Irradiation Conditions

Irradiation Location

Design Temperature

First Cycle Goal

Irradiation Time

Irradiation Charge Number

Holder diameter

Fill Gas

\section{Capsule Fabrication}

\begin{tabular}{|c|c|c|c|c|c|c|c|c|c|c|}
\hline & Drawing & Rev. & Part & Material & Count & Comment & MAT IR & FAB IR & ID & \begin{tabular}{|l|} 
Mass $(\mathrm{g})$ \\
\end{tabular} \\
\hline Housing & X3E020977A634 & A & 1 & Al 6061 & 1 & & 20838 & 20839 & $18-08$ & \\
\hline Housing end cap & X3E020977A634 & A & 2 & Al 4047 & 1 & & 20714 & 20714 & $17-98$ & 0.5149 \\
\hline Foil Spacer & S16-20-ESTEEL & 1 & 2 & Grafoil & 6 & & 19812 & 19812 & 6 Total & 0.0570 \\
\hline Wave Spring & S16-23-ESTEELB & 1 & CM08-L3-S17 & $17-7 \mathrm{SS}$ & 2 & & 20770 & 20770 & 2 Total & 0.2550 \\
\hline \multirow[t]{3}{*}{ Specimen Holder } & \multirow[t]{3}{*}{ S16-22-ESTEEL } & \multirow[t]{3}{*}{1} & \multirow[t]{3}{*}{4} & \multirow[t]{3}{*}{ Al 6061} & \multirow[t]{3}{*}{3} & & \multirow[t]{3}{*}{20825} & \multirow[t]{3}{*}{20785} & $17-05$ & 1.1621 \\
\hline & & & & & & & & & $17-07$ & 1.1585 \\
\hline & & & & & & & & & $17-08$ & 1.1603 \\
\hline Spring Pin & S16-21-ESTEEL & 2 & 91610A207 & $18-8 \mathrm{SS}$ & 3 & & 20548 & 20548 & 3 Total & 0.4070 \\
\hline Chevron & S16-18-FUSSAM01 & 1 & 1 & EuroFer & 18 & 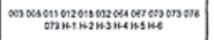 & 20777 & 20777 & 18 Total & 1.9669 \\
\hline \multirow[t]{11}{*}{ Thermometry } & \multirow[t]{11}{*}{ S16-18-FUSSAM01 } & \multirow[t]{11}{*}{1} & \multirow[t]{11}{*}{7} & \multirow[t]{11}{*}{$\mathrm{SiC}$} & \multirow[t]{11}{*}{12} & & \multirow[t]{11}{*}{19759} & \multirow[t]{11}{*}{20840} & 73 & 0.0993 \\
\hline & & & & & & & & & 74 & 0.0987 \\
\hline & & & & & & & & & 75 & 0.1001 \\
\hline & & & & & & & & & 76 & 0.1001 \\
\hline & & & & & & & & & 77 & 0.1005 \\
\hline & & & & & & & & & 78 & 0.1006 \\
\hline & & & & & & & & & 79 & 0.1000 \\
\hline & & & & & & & & & 80 & 0.0980 \\
\hline & & & & & & & & & 81 & 0.1005 \\
\hline & & & & & & & & & 82 & 0.1002 \\
\hline & & & & & & & & & 83 & $\begin{array}{l}0.0988 \\
0.1005\end{array}$ \\
\hline josJ3 & S16-18-FUSSAM01 & 1 & 2 & EuroFer & 20 & & 20778 & 20778 & E279 & 0.2610 \\
\hline & & & & & & & 20778 & 20778 & E280 & 0.2612 \\
\hline & & & & & & & $\begin{array}{l}20778 \\
20778\end{array}$ & $\begin{array}{l}20778 \\
20778\end{array}$ & $\begin{array}{l}\text { E281 } \\
\text { F282 }\end{array}$ & 0.2613 \\
\hline & & & & & & & $\begin{array}{l}20778 \\
20778\end{array}$ & $\begin{array}{l}20778 \\
20778\end{array}$ & \begin{tabular}{|l|}
$E 282$ \\
$E 283$
\end{tabular} & $\begin{array}{l}0.2603 \\
0.2581\end{array}$ \\
\hline & & & & & & & 20778 & 20778 & E284 & 0.2626 \\
\hline & & & & & & & 20778 & 20778 & E285 & 0.2625 \\
\hline & & & & & & & 20778 & 20778 & E286 & 0.2604 \\
\hline & & & & & & & $\begin{array}{l}20778 \\
20778\end{array}$ & $\begin{array}{r}20778 \\
20778\end{array}$ & $\begin{array}{l}E 287 \\
E 288\end{array}$ & 0.2580 \\
\hline & & & & & & & 20778 & $\begin{array}{l}20778 \\
20778\end{array}$ & $\begin{array}{l}\text { E288 } \\
\text { E289 }\end{array}$ & $\begin{array}{l}0.2626 \\
0.2614\end{array}$ \\
\hline & & & & & & & 20778 & 20778 & E290 & 0.2592 \\
\hline & & & & & & & 20841 & 20841 & YB28 & 0.2681 \\
\hline & & & & & & & 20841 & 20841 & YB29 & 0.2700 \\
\hline & & & & & & & 20841 & 20841 & YB30 & 0.2649 \\
\hline & & & & & & & 20841 & 20841 & YB31 & 0.2667 \\
\hline & & & & & & & 20841 & 20841 & YB32 & 0.2688 \\
\hline MPC1 & S16-18-FUSSAM01 & 1 & 5 & EuroFer & 12 & & 20841 & 20841 & \begin{tabular}{|r|}
$\mathrm{B} 54-08$ \\
$\mathrm{~F} 336$
\end{tabular} & 0.2675 \\
\hline & & & & & & & 20780 & 20780 & $\begin{array}{l}\text { E336 } \\
\text { E337 }\end{array}$ & 0.1210 \\
\hline & & & & & & & & & E338 & 0.1130 \\
\hline & & & & & & $\therefore$ & & & E339 & 0.1130 \\
\hline & & & & & & & & & E340 & 0.1120 \\
\hline MPC2 & S16-18-FUSSAM01 & 1 & 6 & EuroFer & 12 & & 20781 & 20781 & $\begin{array}{l}\text { E341 } \\
\text { E436 }\end{array}$ & 0.1100 \\
\hline & & & & & & & & & E437 & 0.2590 \\
\hline & & & & & & & & & E438 & 0.2550 \\
\hline & & & & & & & & & E439 & 0.2590 \\
\hline & & & & & & & & & E440 & 0.2550 \\
\hline & & & & & & & & & $\begin{array}{r}\text { E441 } \\
\text { Total Mass }\end{array}$ & $\begin{array}{r}0.2600 \\
14.8338 \\
\end{array}$ \\
\hline & & & & & & & & & Specimen Mass & 6.9548 \\
\hline Assembly & & & & & & & & & Intemal Mass & 14.3189 \\
\hline & Drawing & Rev. & Con & & & & & & & \\
\hline Assembly Drawing & S16-20-ESTEEL & 1 & & & & & & & & \\
\hline Welding \& Cleaning & X3E020977A633 & 0 & & & & & & & & \\
\hline Fill Gas & Helium & & & & & & & & & \\
\hline
\end{tabular}

\begin{tabular}{|c|c|c|}
\hline \multirow{2}{*}{ Approvals } & \multirow[b]{2}{*}{ Request } & \multirow[b]{2}{*}{ Build } \\
\hline & & \\
\hline Performed by: & & \\
\hline Checked by: & & \\
\hline
\end{tabular}

$\mathrm{mm}\left(0.3622\right.$ in) at $20^{\circ} \mathrm{C}$

Helium

\begin{tabular}{rr} 
& TRRH \\
\hline & 375 \\
\hline 2 & 477 \\
\hline & cyc. \\
\hline & N/A \\
\hline $9.20 \quad m m\left(0.3622\right.$ in) at $20^{\circ} \mathrm{C}$ \\
Helium \\
\hline
\end{tabular}


ES07

(17-05)

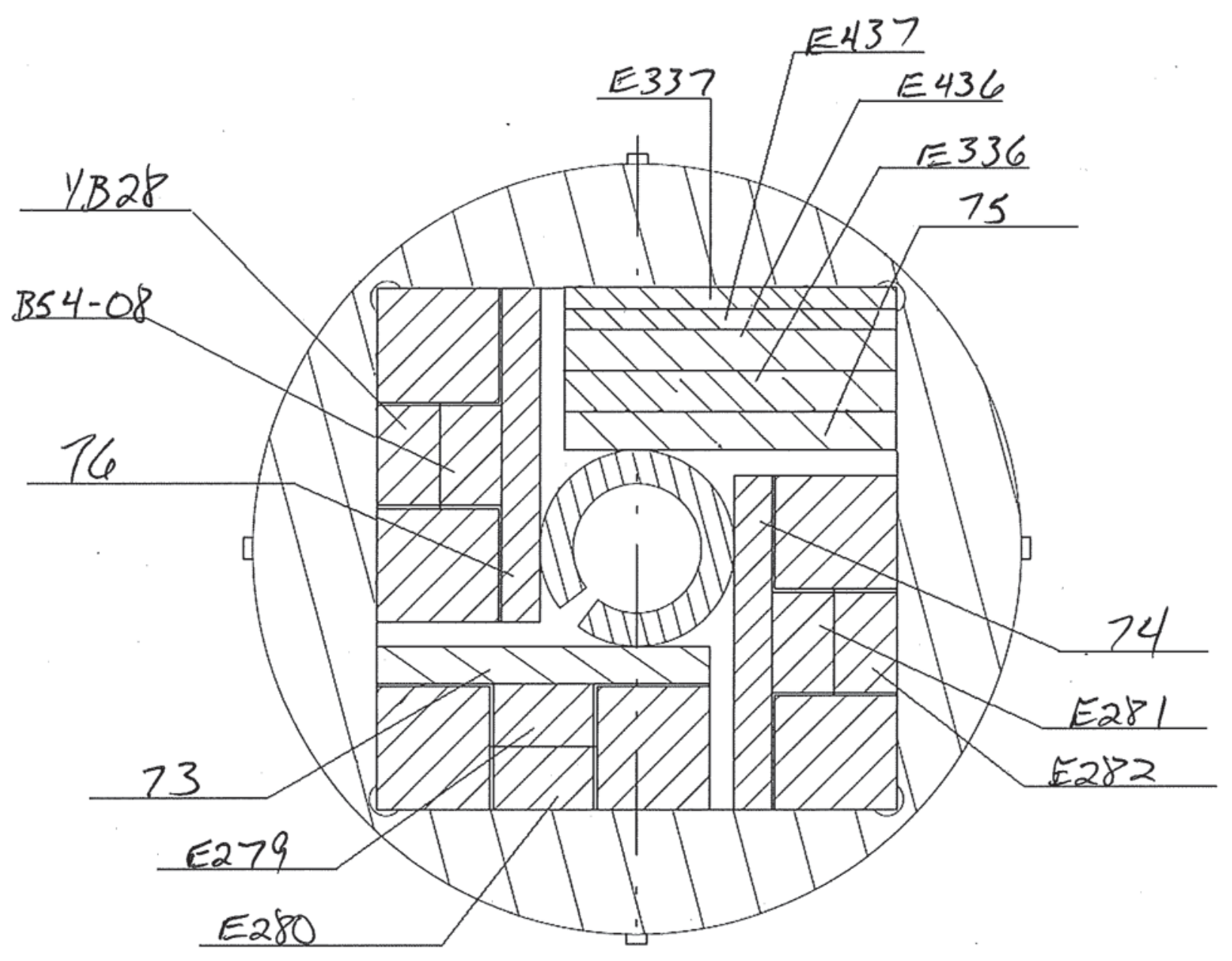


ES07

(17-07)

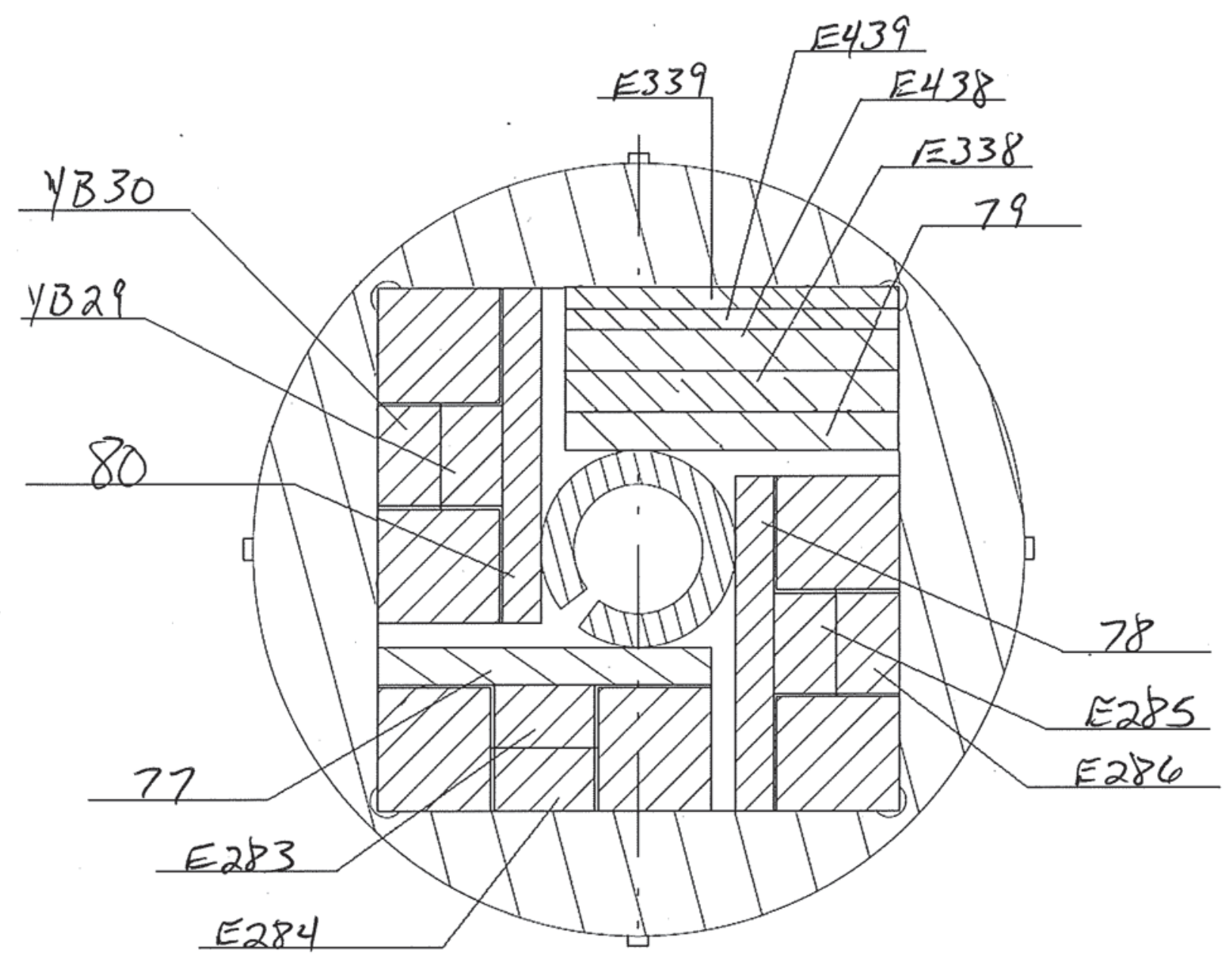


ES07

(17-08)

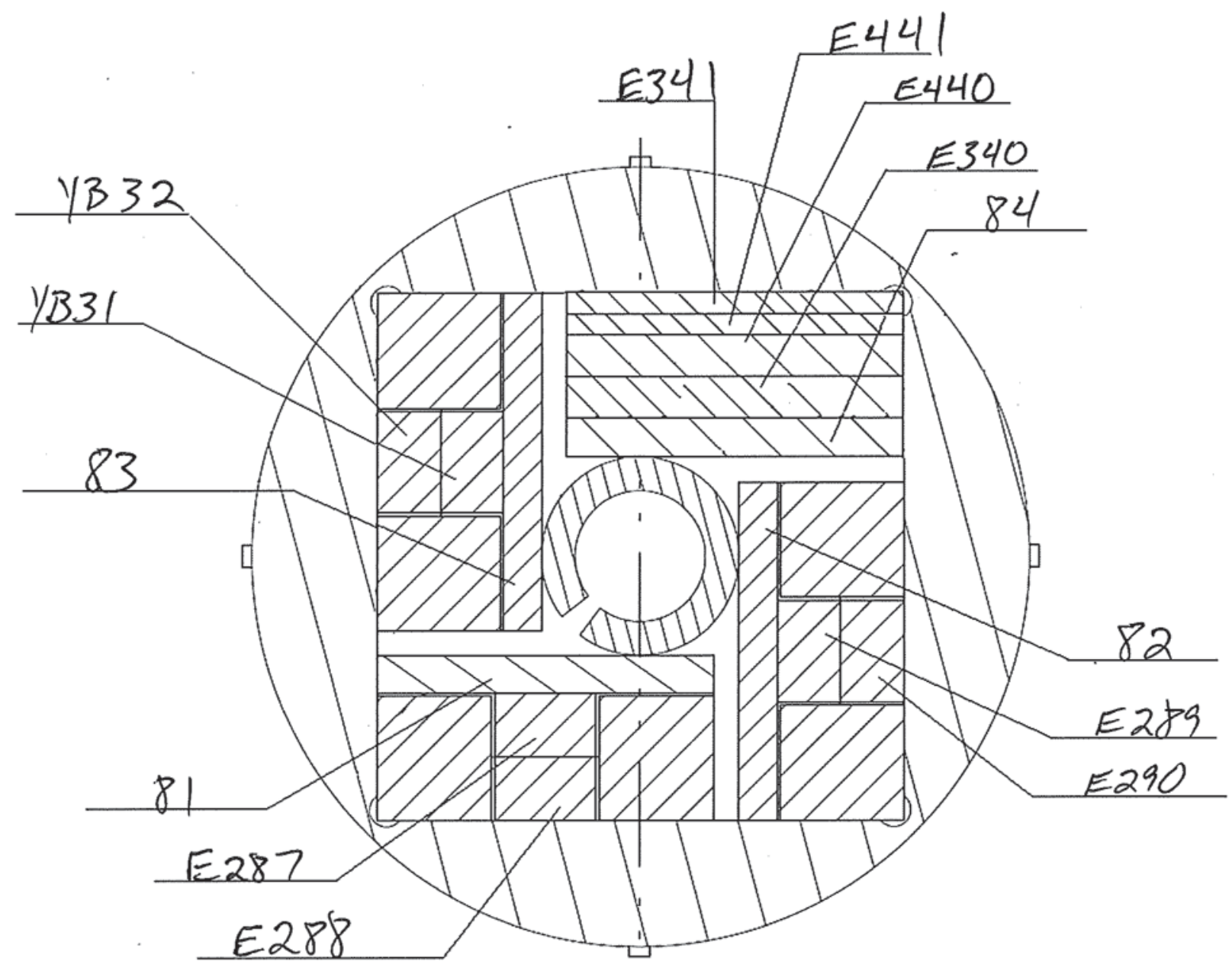




\begin{tabular}{|c|c|c|c|c|c|}
\hline \multicolumn{6}{|c|}{ ES07 } \\
\hline \multirow{3}{*}{$\begin{array}{r}\text { Design Diameter (mm) } \\
9\end{array}$} & $\operatorname{Tavg}\left({ }^{\circ} \mathrm{C}\right)$ & $\operatorname{Tavg}\left({ }^{\circ} \mathrm{C}\right)$ & & & \\
\hline & 388 & 374 & & & \\
\hline & Inner & Outer & & & Specimen Type \\
\hline \multirow{4}{*}{ Upper $(X-Y c)$} & E279 & E280 & 12 & 64 & SSJ3 (E) \\
\hline & E281 & E282 & 3 & 67 & SSJ3 (E) \\
\hline & E336 & E337 & E436 & E437 & MPC1 (E3) / MPC2 (E4) \\
\hline & B54-08 & YB28 & $\mathrm{H} 1$ & $\mathrm{H} 2$ & SSJ3 \\
\hline \multirow{4}{*}{$\operatorname{Mid}(X-Y c)$} & E283 & E284 & 11 & 70 & SSJ3 (E) \\
\hline & E285 & E286 & 8 & 73 & SSJ3 (E) \\
\hline & E338 & E339 & E438 & E439 & MPC1 (E3) / MPC2 (E4) \\
\hline & YB29 & YB30 & $\mathrm{H} 3$ & $\mathrm{H} 4$ & SSJ3 \\
\hline \multirow{4}{*}{ Lower $(X-Y c)$} & E287 & E288 & 18 & 76 & SSJ3 (E) \\
\hline & E289 & E290 & 32 & 79 & SSJ3 (E) \\
\hline & E340 & E341 & E440 & E441 & MPC1 (E3) / MPC2 (E4) \\
\hline & YB31 & YB32 & H5 & $\mathrm{H} 6$ & SSJ3 \\
\hline
\end{tabular}




\section{RABBIT ASSEMBLY: $E S \varnothing 7$ HOUSING}

$\operatorname{ESOT}$

SN / IR NUMBER:

$18-\not 8 / 20839$

INNER

DIAMETER:

$\phi 9.52$

HOLDER(S)

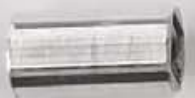

SN / IR NUMBER:

$17-\not 55 / 20785$

OUTER

DIAMETER:

$\phi 9.15$
SN / IR NUMBER:

$17-\not 7 / 2 \varnothing 785$

OUTER

DIAMETER:

$\varnothing 9.15$

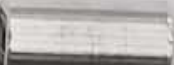

SN / IR NUMBER:

$17-\varnothing 8 / 20785$

OUTER

DIAMETER:

$\varnothing 9.15$ 


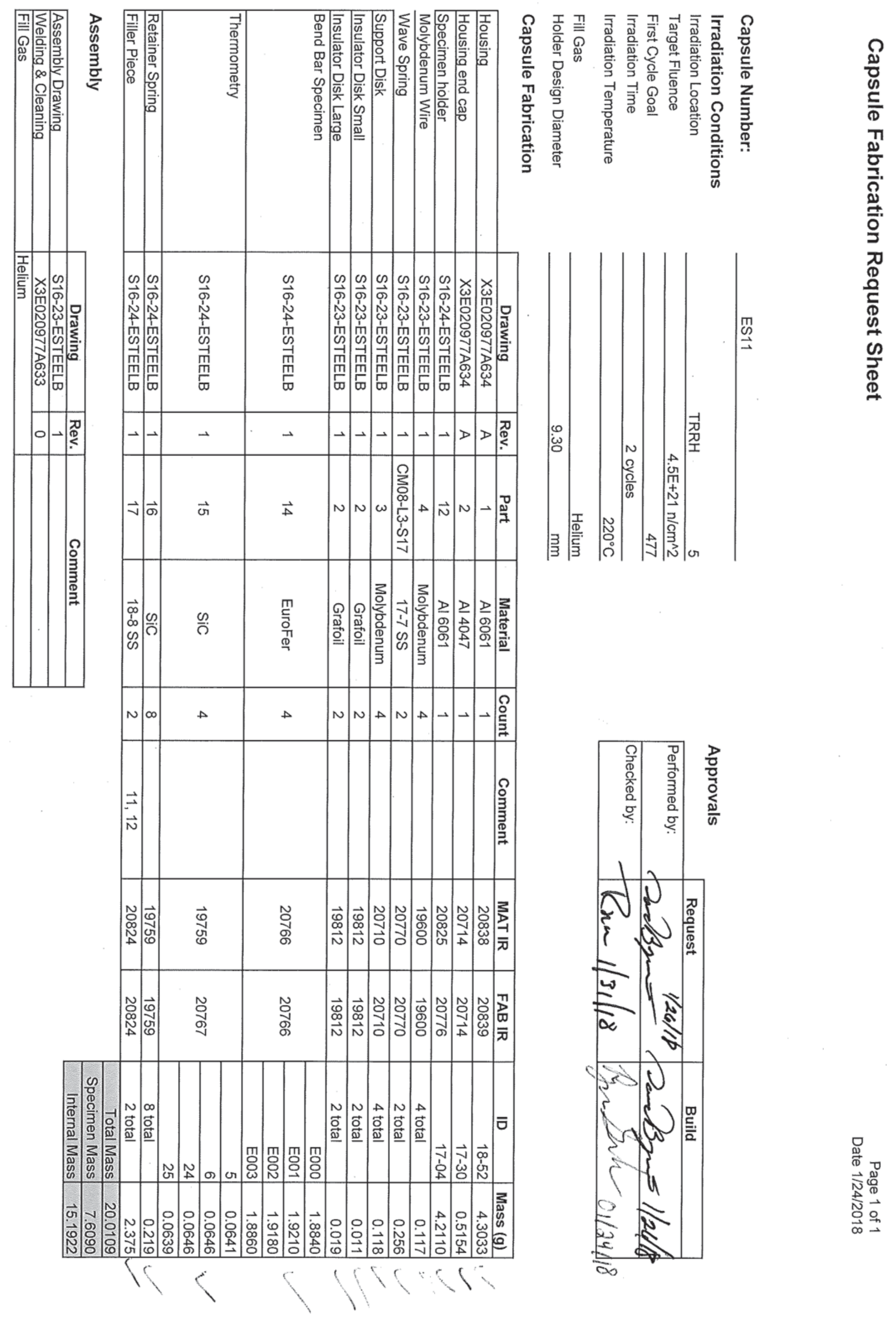


ES11

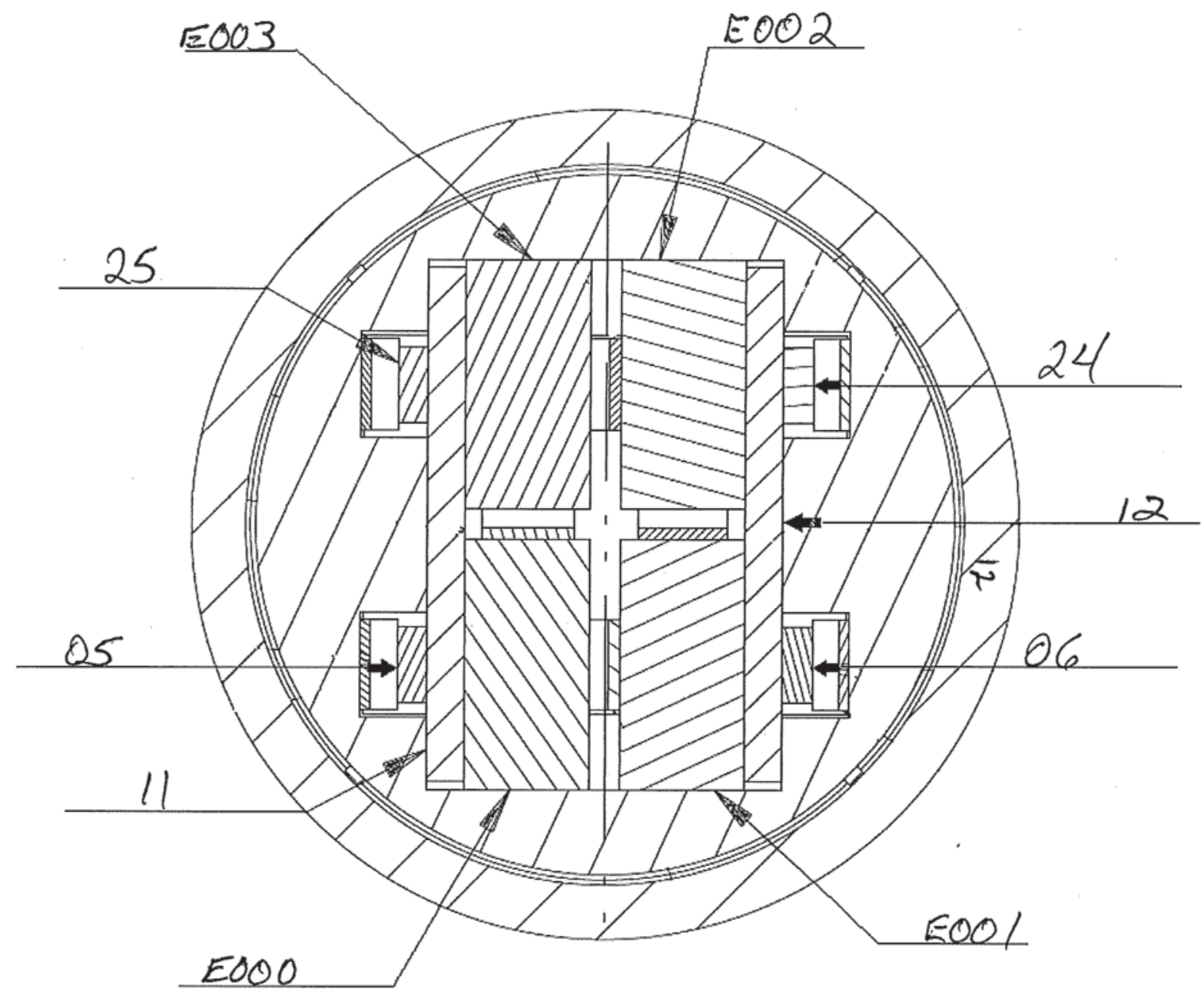


RABBIT ASSEMBLY: ES II HOUSING

ES19 mand

SN / IR NUMBER:

$18-52 / 2 \not 839$

INNER

DIAMETER:

$\phi 9.53$

HOLDER(S)

SN / IR NUMBER:

$17-\phi 4 / 2 \varnothing 77 \underline{6}$

OUTER DIAMETER:

$\varnothing 9.43$ 


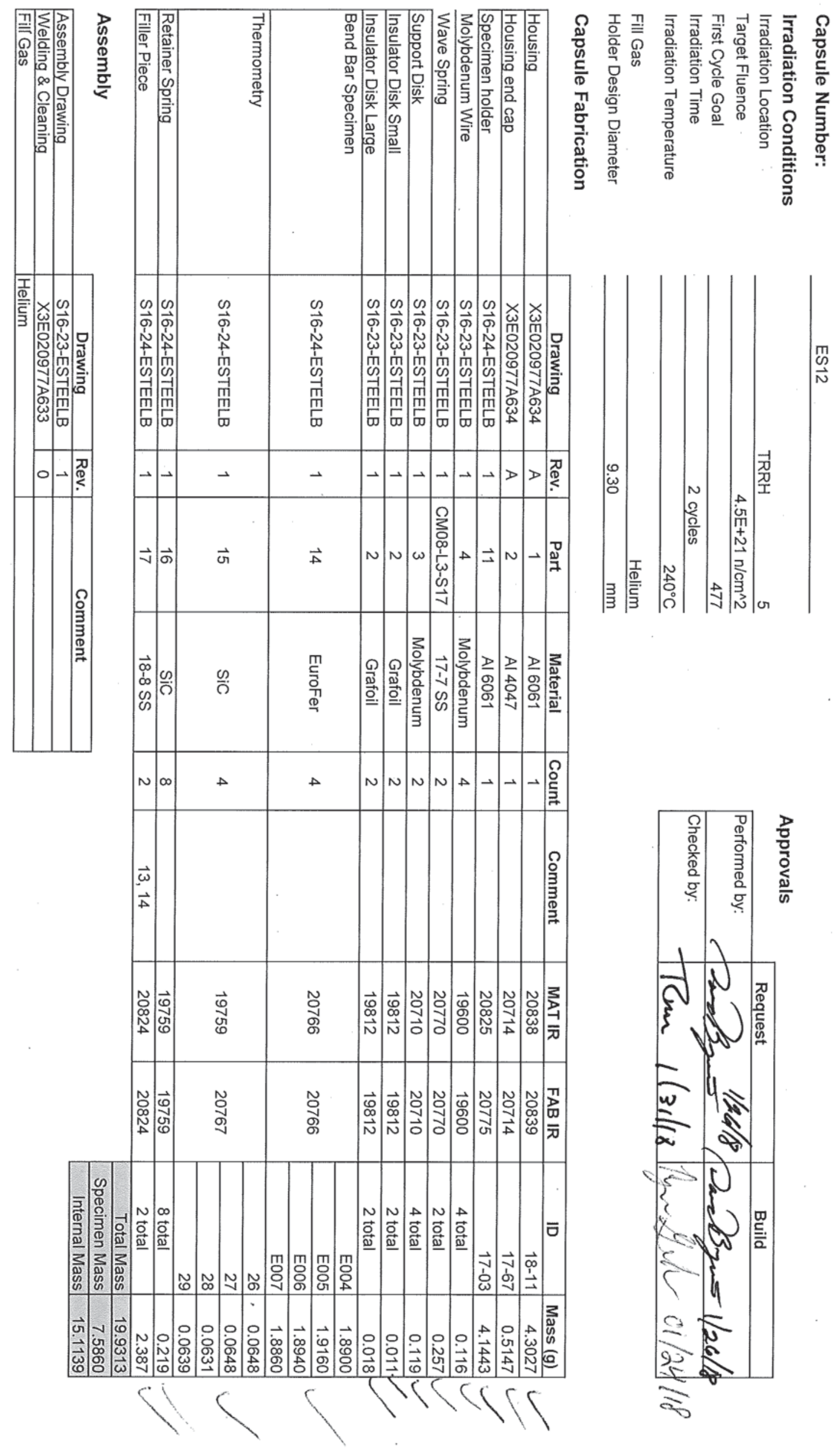


ES12

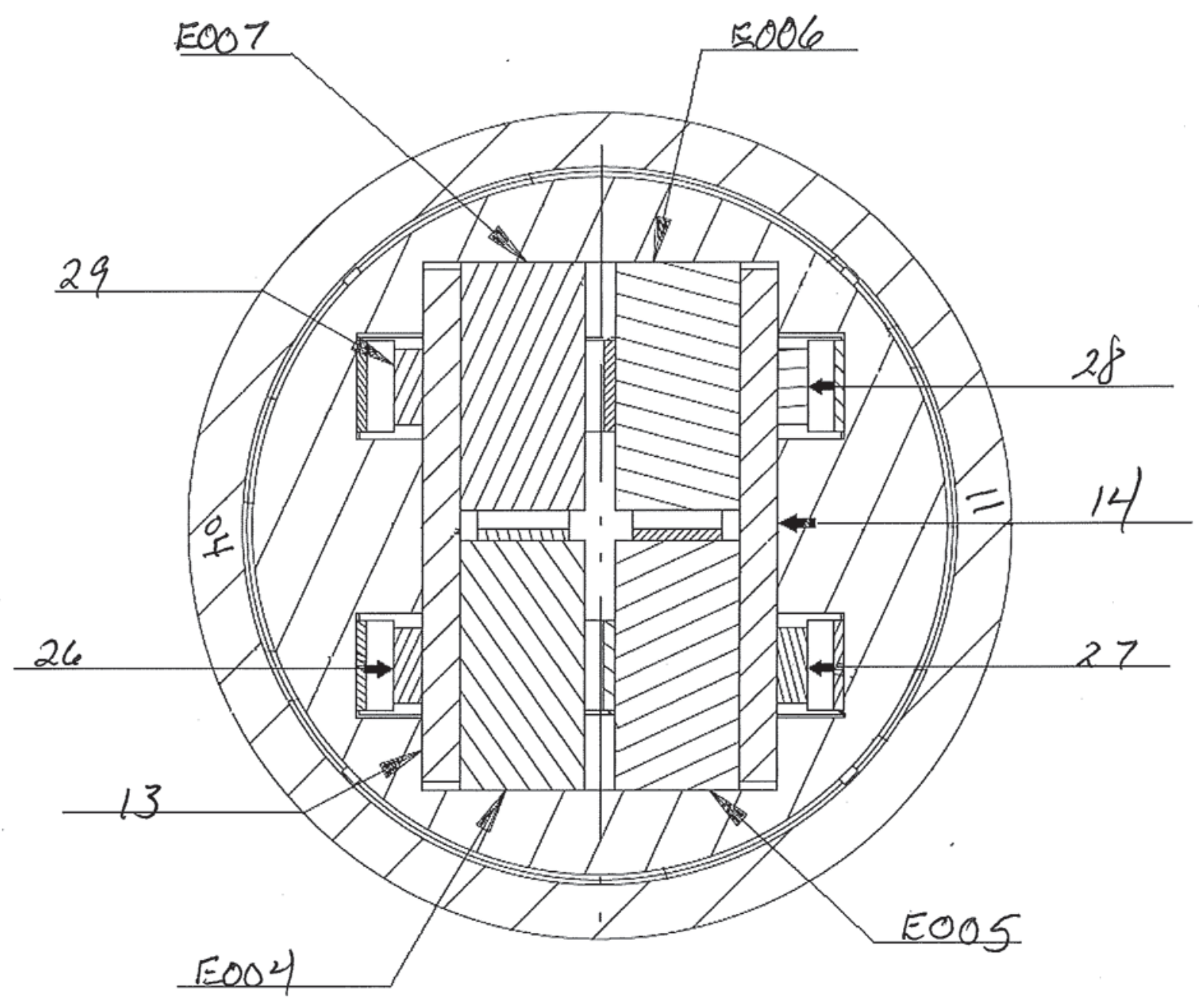




\section{RABBIT ASSEMBLY: ES/2 HOUSING}

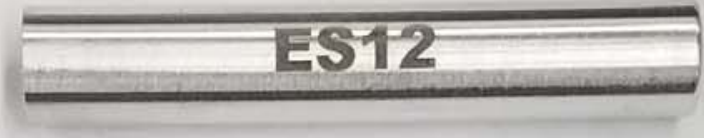

SN / IR NUMBER:

$18-11 / 28839$

INNER

DIAMETER:

$\phi 9.51$

HOLDER(S)

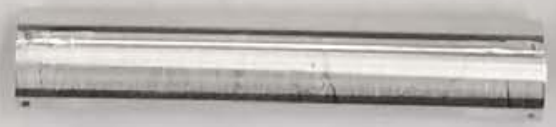

SN / IR NUMBER:

$17-\not 3 / 2 \varnothing 775$

OUTER

DIAMETER:

$\phi 9.4 \phi$ 


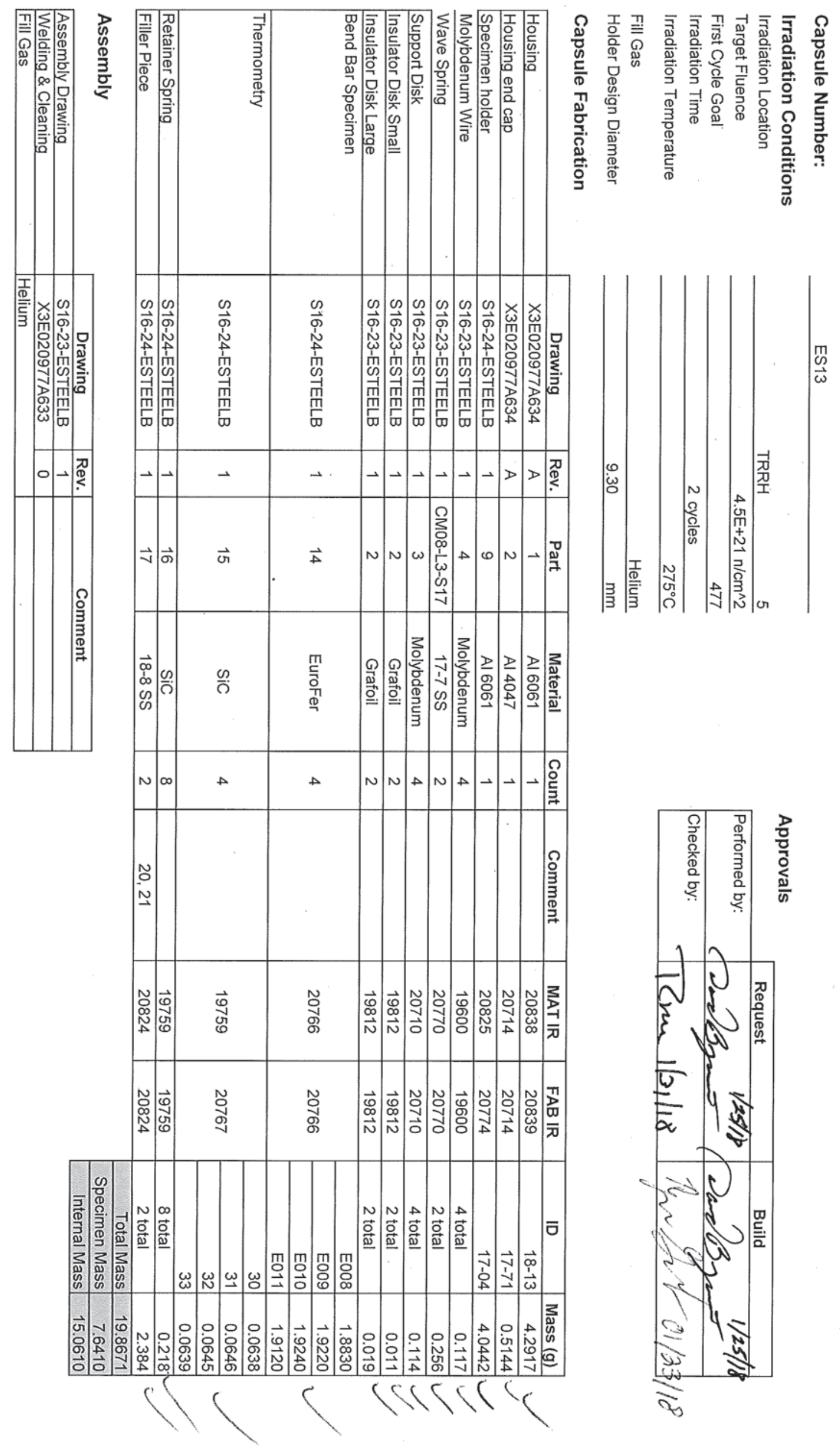


ES13

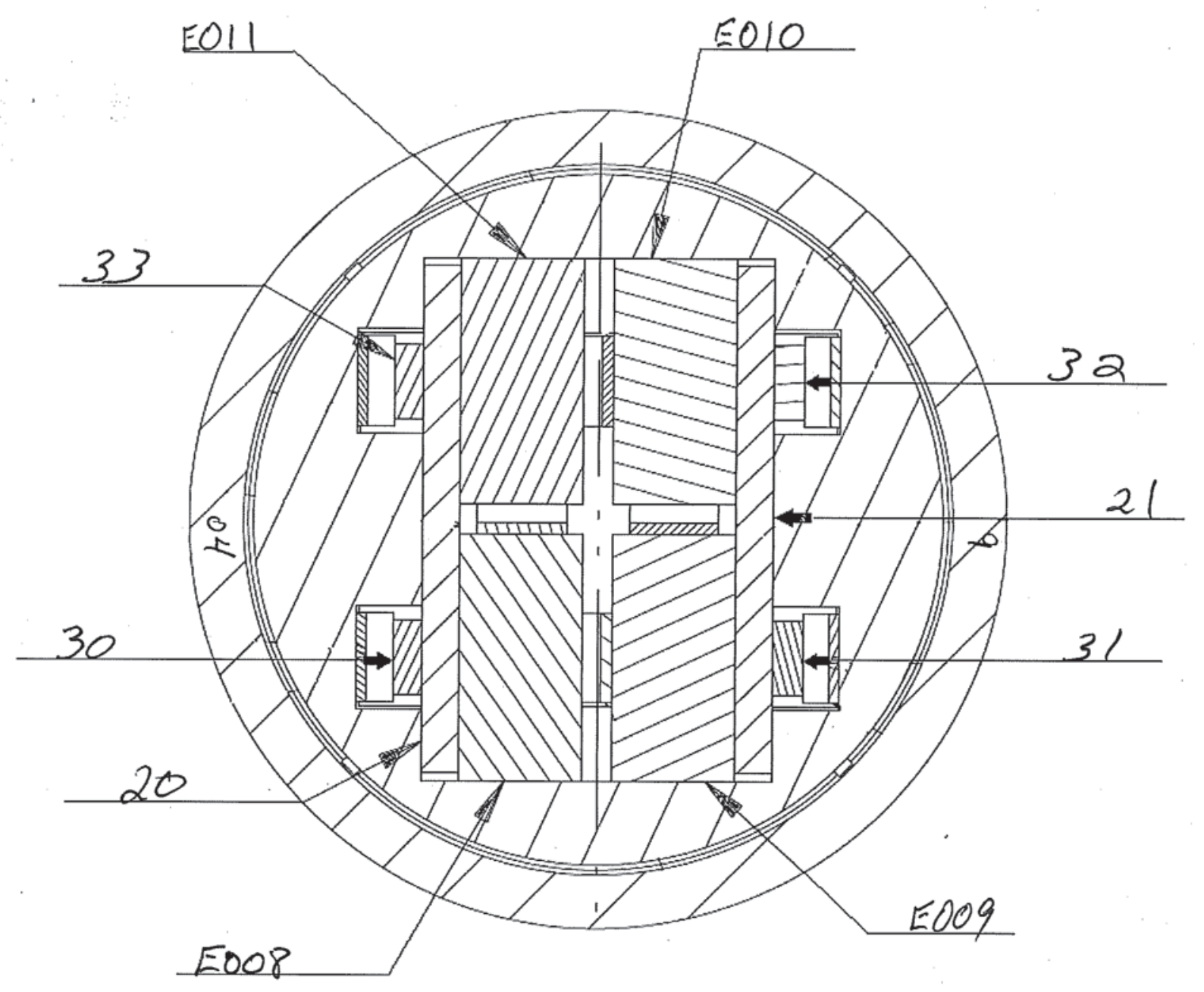


RABBIT ASSEMBLY: ES/3 HOUSING

ES13

SN / IR NUMBER:

$18-13 / 2 \not 8839$

INNER

DIAMETER:

$\phi \underline{q .53}$

HOLDER(S)

SN / IR NUMBER:

$17-\varnothing 4 / 28774$

OUTER

DIAMETER:

$\phi 9.35$ 

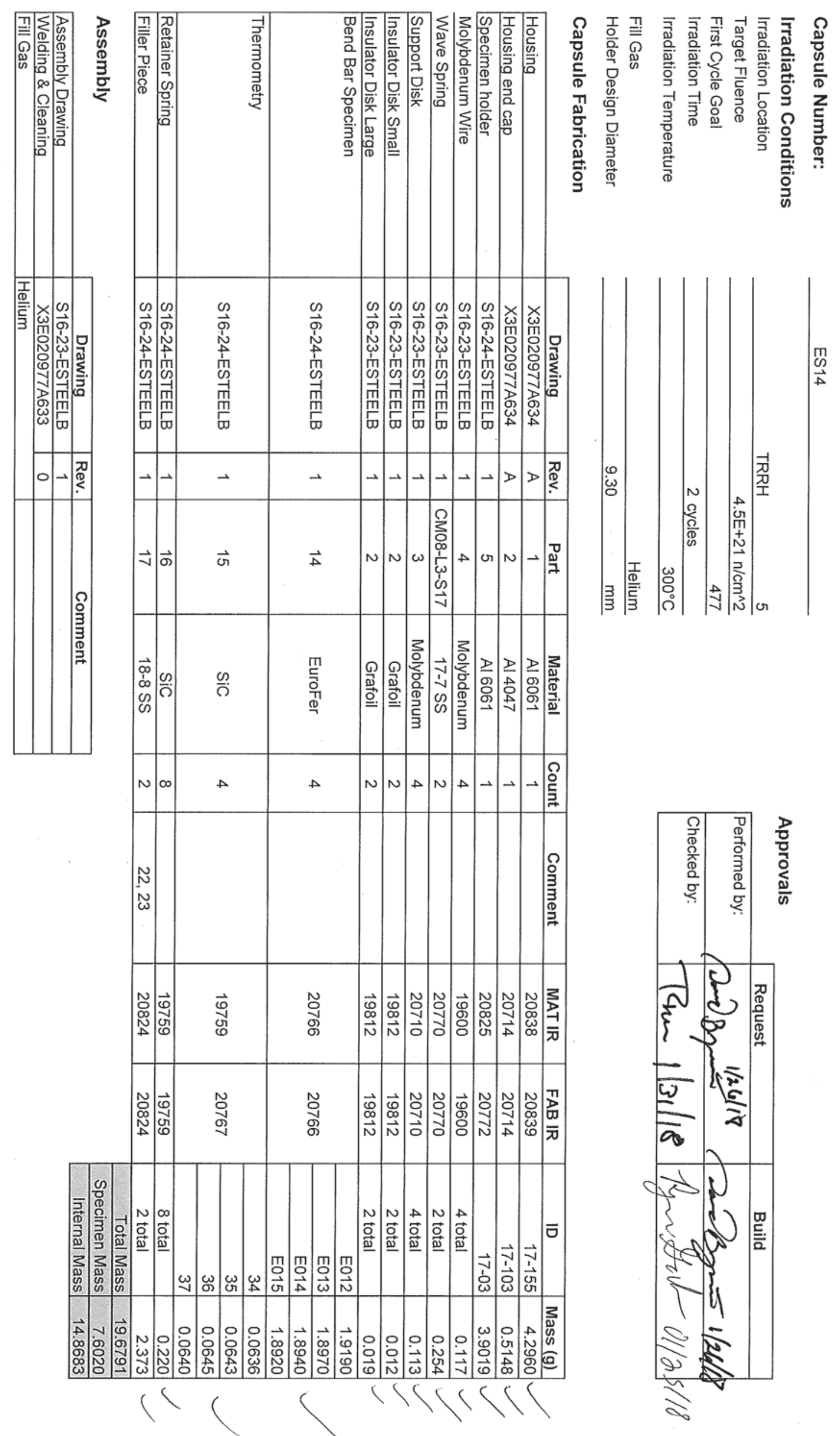
ES14

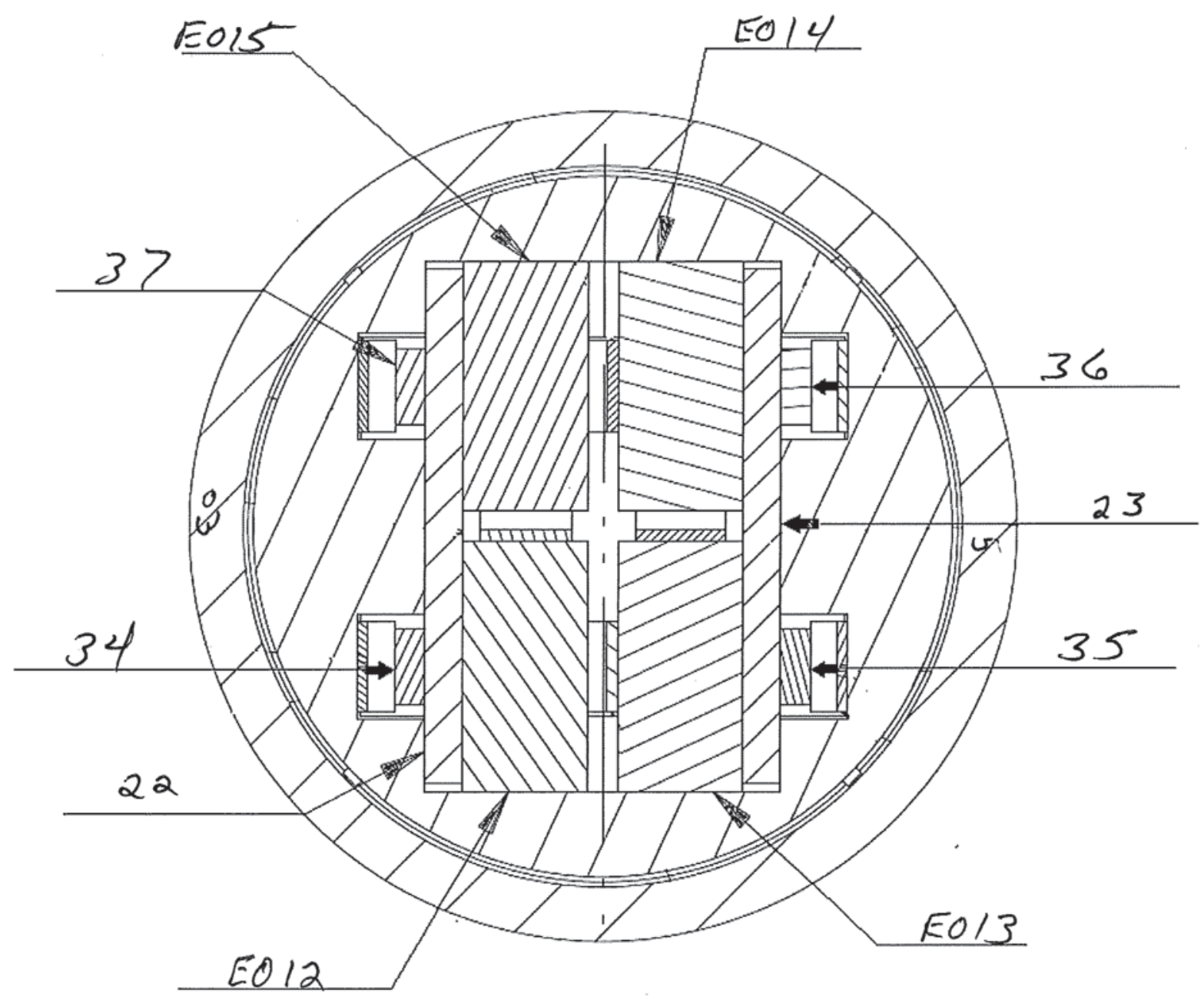




\section{RABBIT ASSEMBLY: ES14 HOUSING}

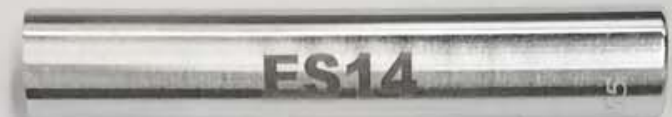

SN / IR NUMBER:

$$
17-155 / 2 \$ 839
$$

INNER

DIAMETER:

$\varnothing 9.53$

\section{$\operatorname{HOLDER}(\mathrm{S})$}

SN / IR NUMBER:

$17-\not 83 / 28772$

OUTER

DIAMETER:

I. 28 


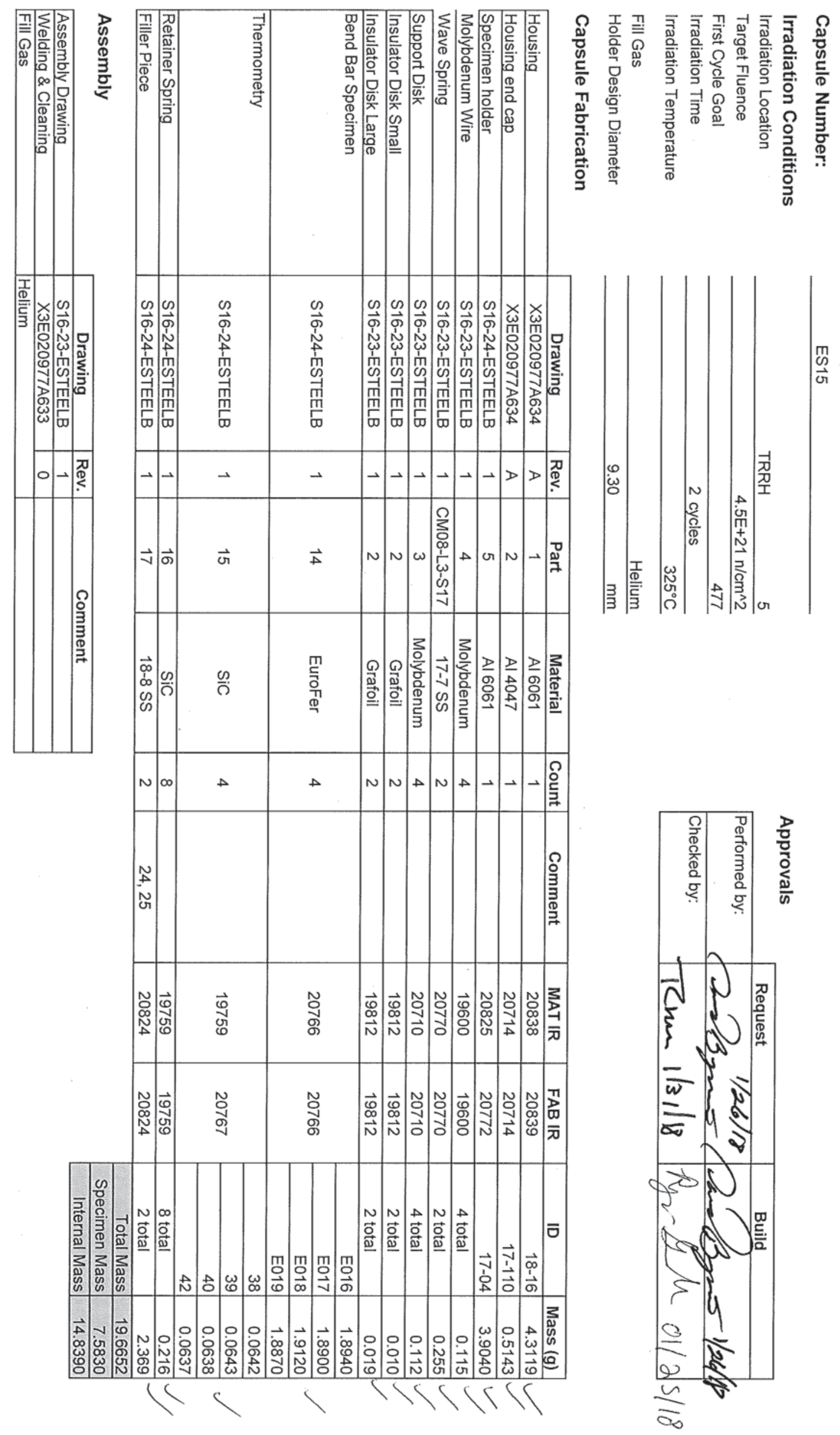


ES15

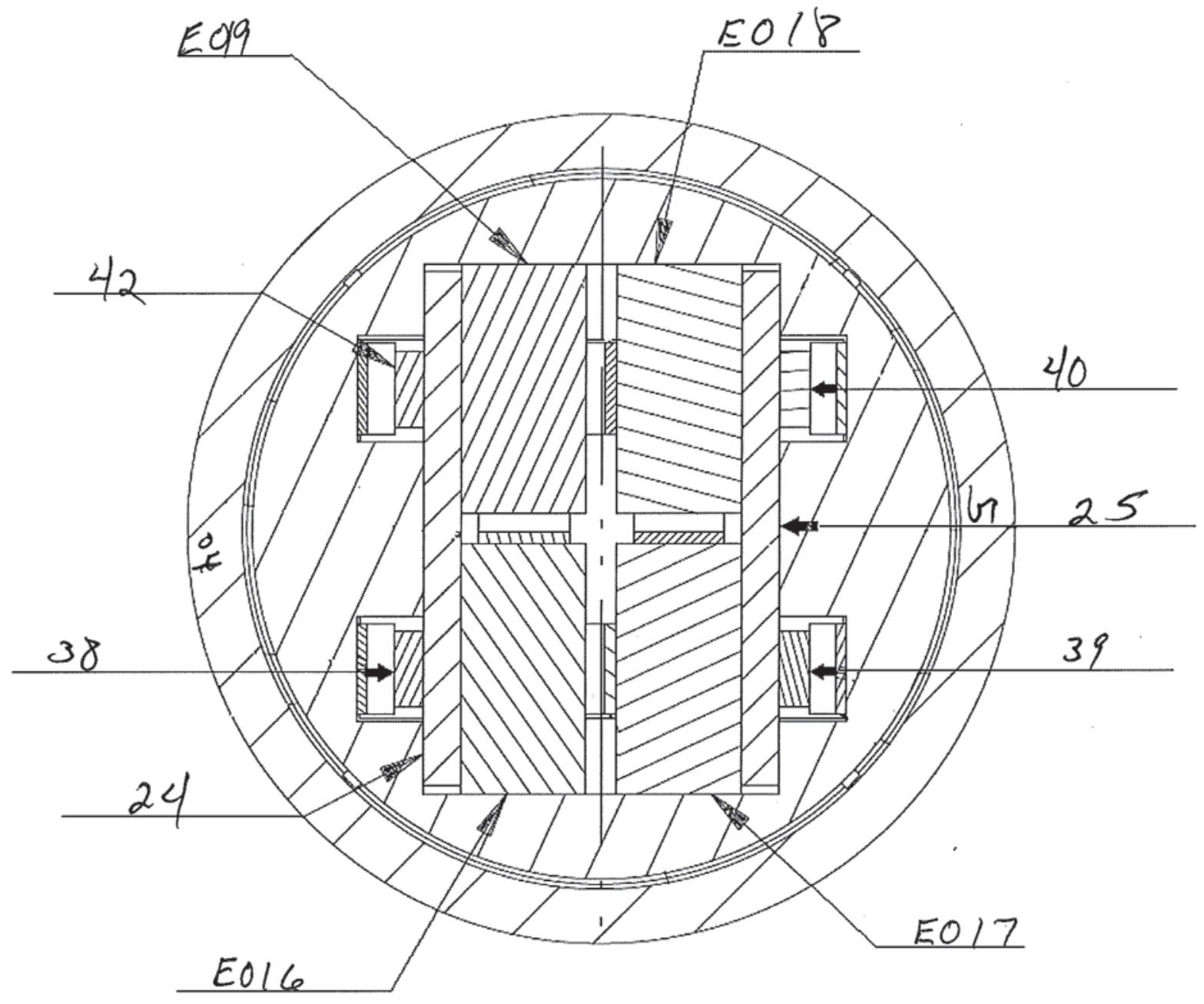




\section{RABBIT ASSEMBLY: ES I 5 HOUSING}

\section{ES15}

SN / IR NUMBER:

$18-16 / 2083 \underline{9}$

INNER

DIAMETER:

$\varnothing 9.52$

$\operatorname{HOLDER}(\mathrm{S})$

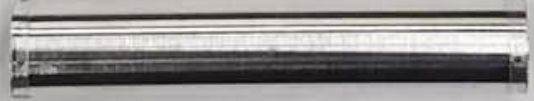

SN / IR NUMBER:

$17-94 / 28772$

OUTER

DIAMETER:

$\varnothing 9.27$ 


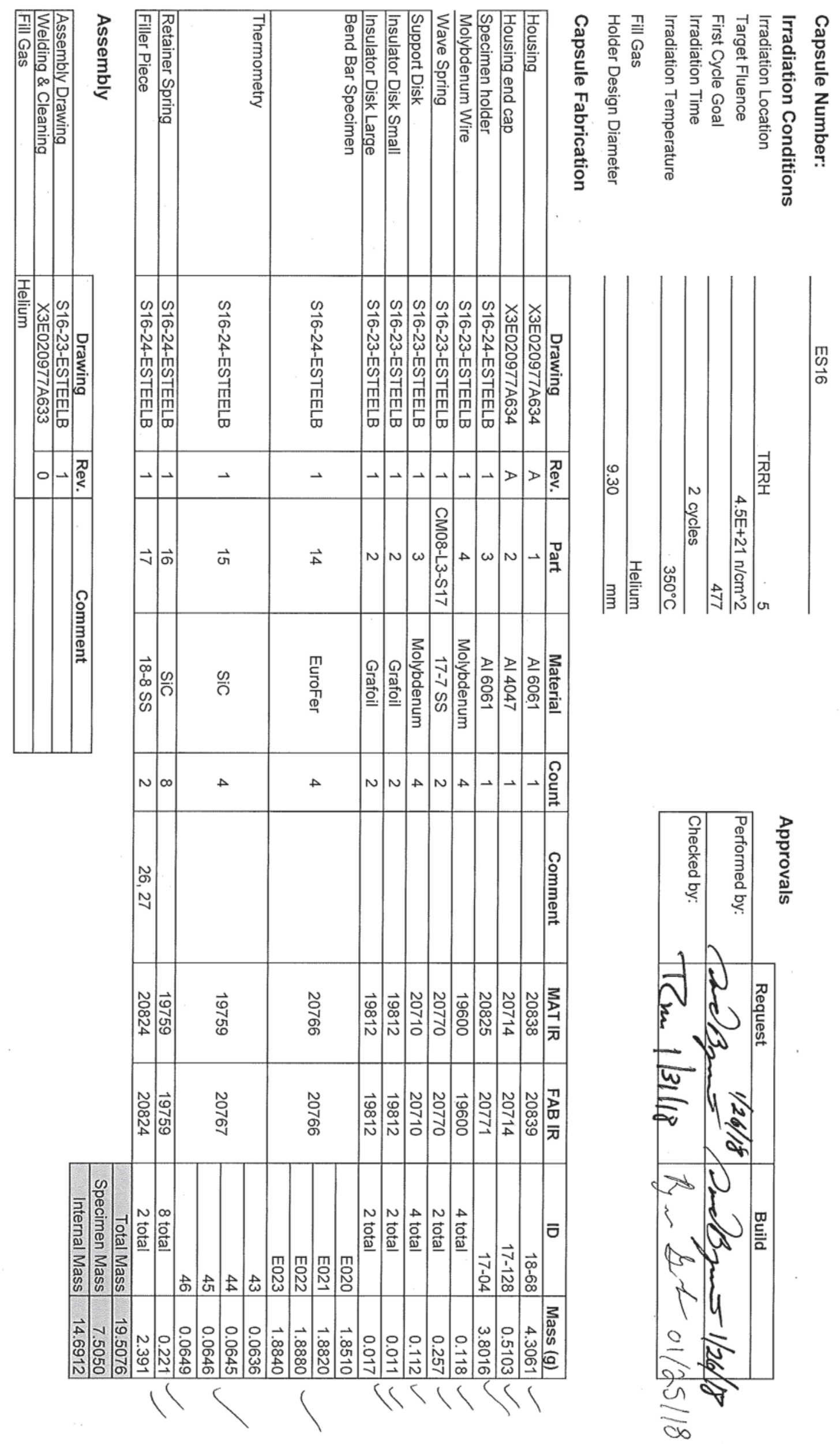


ES16

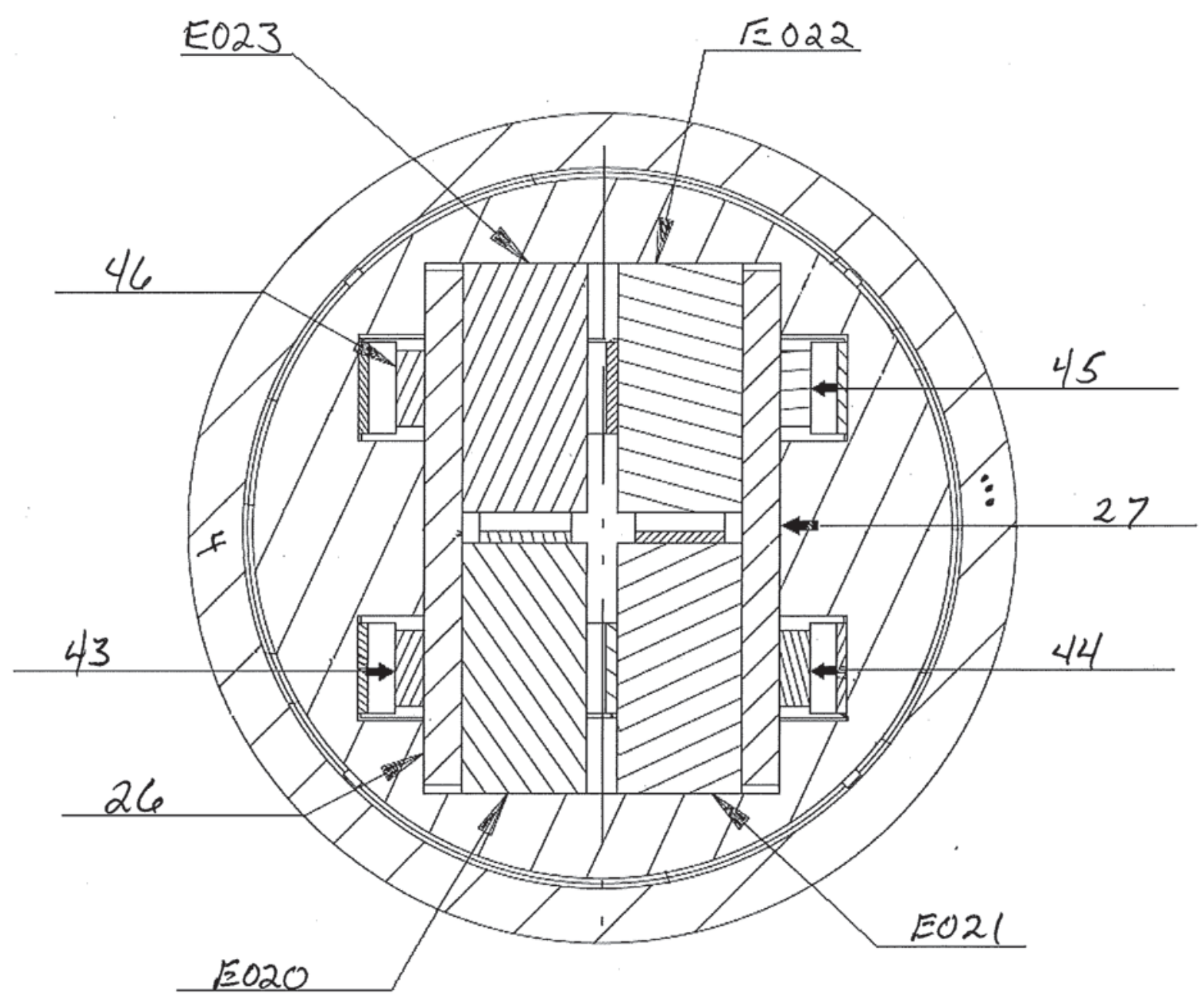




\section{RABBIT ASSEMBLY: ESI 6 HOUSING}

ES16

SN / IR NUMBER:

$18-\underline{68 / 28839}$

INNER

DIAMETER:

$\phi 9.53$

HOLDER(S)

SN / IR NUMBER:

$17-\varnothing 4 / 2 \varnothing 771$

OUTER

DIAMETER:

$\phi \underline{q} .2 \phi$ 


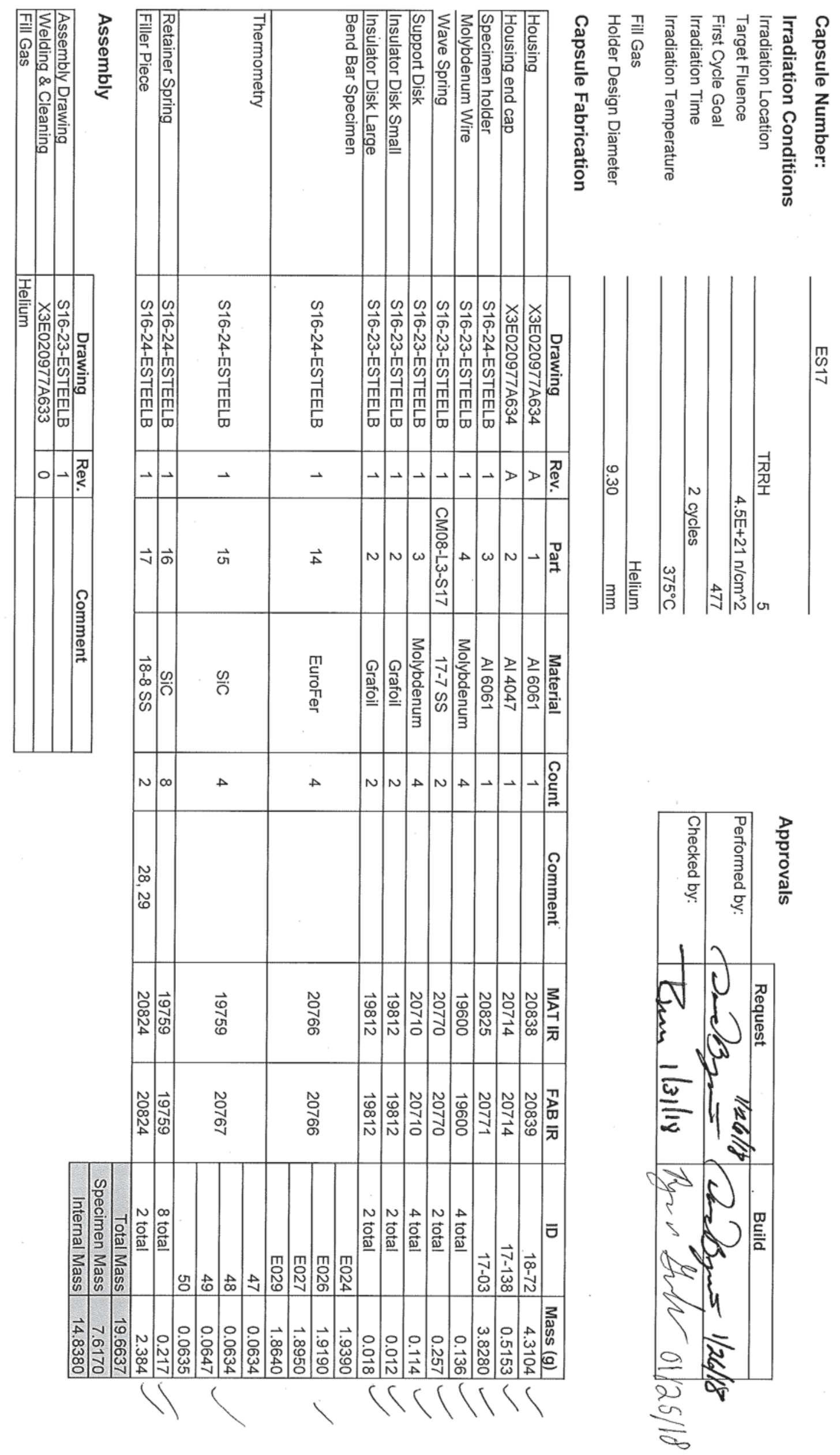


ES17

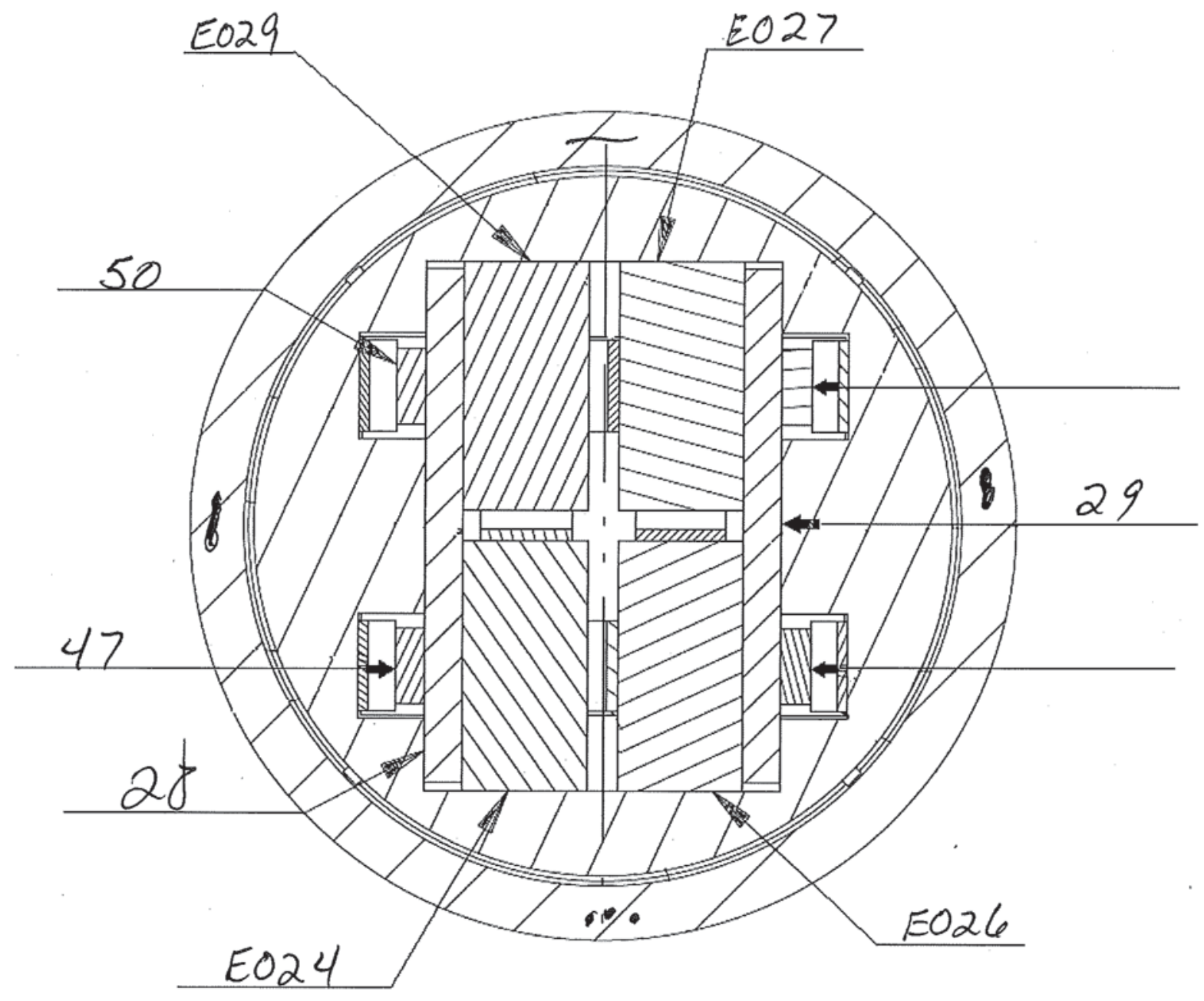


RABBIT ASSEMBLY: ES IF HOUSING

SN / IR NUMBER:

$18-72 / 288839$

INNER

DIAMETER:

$\phi 9.53$

HOLDERS)

SN / IR NUMBER:

$17-83 / 28771$

OUTER

DIAMETER:

$\phi 9.22$ 
Capsule Number:

Irradiation Conditions

Irradiation Location

Design Temperature

First Cycle Goal

Irradiation Time

Irradiation Charge Number

Holder diameter

Fill Gas

Capsule Fabrication

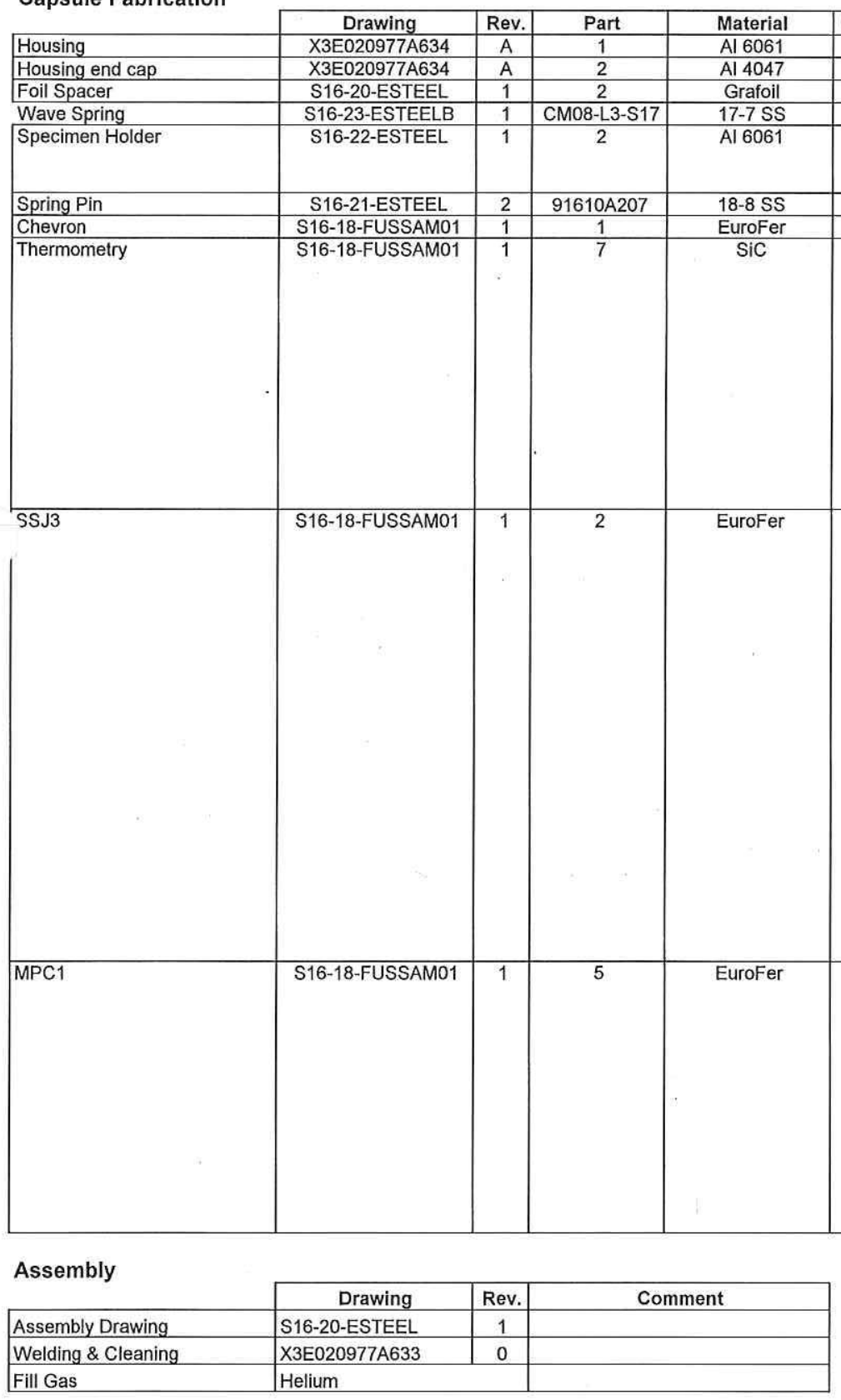

\section{Approvals}

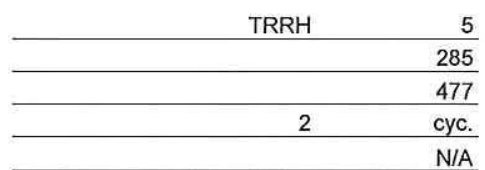

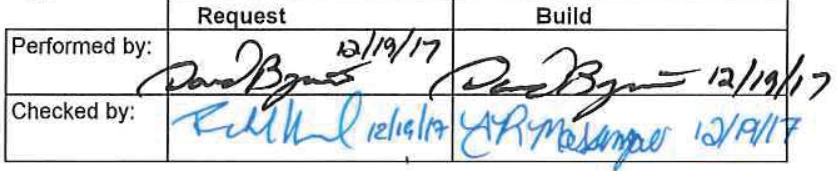

9.25

$\mathrm{mm}(0.3642 \mathrm{in})$ at $20^{\circ} \mathrm{C}$ Helium

\begin{tabular}{l|l} 
Count & Comment
\end{tabular}

\begin{tabular}{l|l|l} 
MAT IR & FAB IR & ID \\
\hline
\end{tabular}

\begin{tabular}{|c|c|r|r|}
\hline 20838 & 20839 & ID & Mass $(\mathrm{g})$ \\
\hline
\end{tabular}

\begin{tabular}{l|l|r|r|}
20714 & 20714 & $17-101$ & 0.5173 \\
\hline 19812 & 19812 & 6701 & 0.0550 \\
\hline
\end{tabular}

\begin{tabular}{lll|}
\hline 9812 & 6 Total & 0.0550 \\
\hline
\end{tabular}

\begin{tabular}{|l|r|r|r|}
20825 & 20770 & 2 Total & 0.2570 \\
\hline
\end{tabular}

\begin{tabular}{l|r|r|r|}
20825 & 20783 & $17-05$ & 1.2254 \\
\cline { 3 - 4 } & & $17-06$ & 1.2279 \\
\cline { 2 - 3 } & $17-09$ & 1.2262 \\
\hline
\end{tabular}

\begin{tabular}{|c|c|c|c|c|}
\hline & & & & 1.2202 \\
\hline & 20548 & 20548 & 3 Total & 0.4100 \\
\hline Parts 1-20 & 20777 & 20777 & 20 Total & 2.1498 \\
\hline
\end{tabular}

\begin{tabular}{l|l|l}
\hline 12 & Parts 1-20 & 2077 \\
\hline
\end{tabular}

\begin{tabular}{l|l}
19759 & 20657
\end{tabular}

20

0

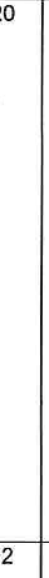

12

\begin{tabular}{l|l}
\hline & 20780
\end{tabular}

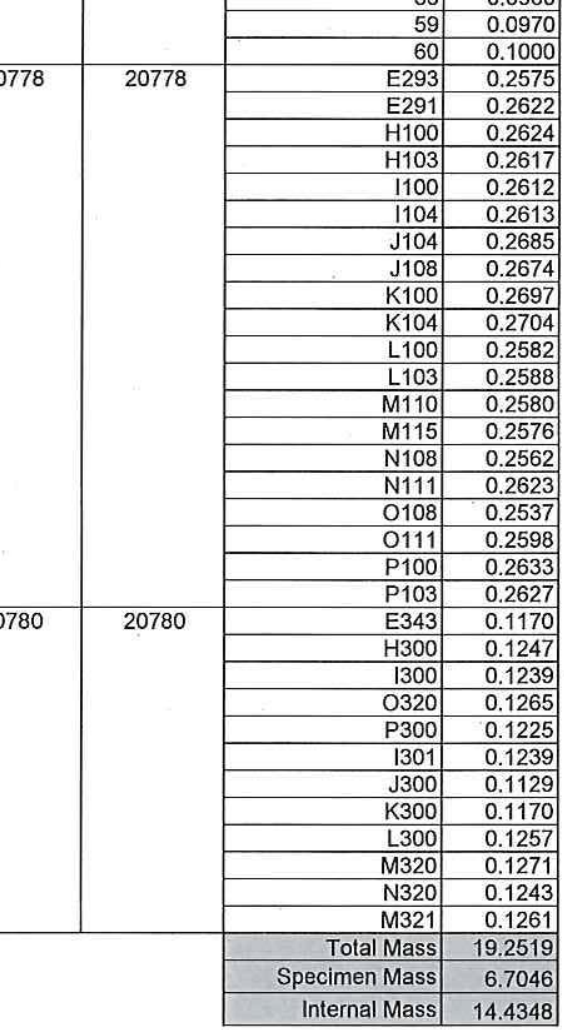

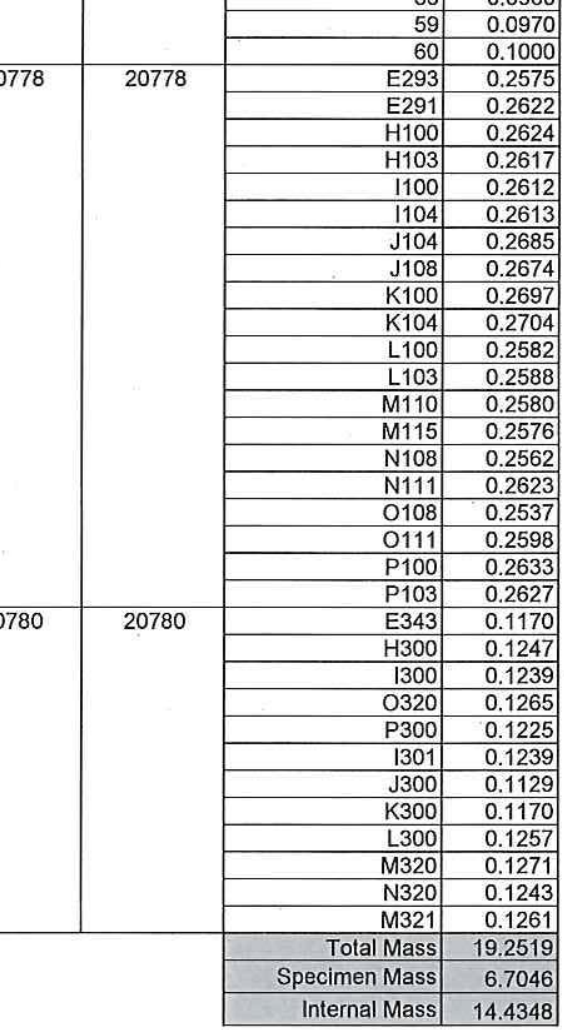

20780

\begin{tabular}{|l|}
20 Tot \\
\hline \\
\hline \\
\hline
\end{tabular}

.

\begin{tabular}{l|l|}
\hline 50 & 0.0980 \\
\hline 51 & 0.1000 \\
\hline 52 & 0.0980 \\
\hline
\end{tabular}

\begin{tabular}{l|l}
53 & 0.0980 \\
\hline 54 & 0.0990 \\
\hline
\end{tabular}

\begin{tabular}{l|l|}
54 & 0.0990 \\
\hline 55 & 0.0980 \\
\hline
\end{tabular}

\begin{tabular}{l|l|}
56 & 0.0970 \\
\hline 57
\end{tabular}

\begin{tabular}{l|l}
57 & 0.0970 \\
\hline 58 & 0.0960 \\
\hline
\end{tabular}

\begin{tabular}{|l|l|}
59 & 0.0970 \\
\hline
\end{tabular} 


\section{ES21}

17-05

(Top)

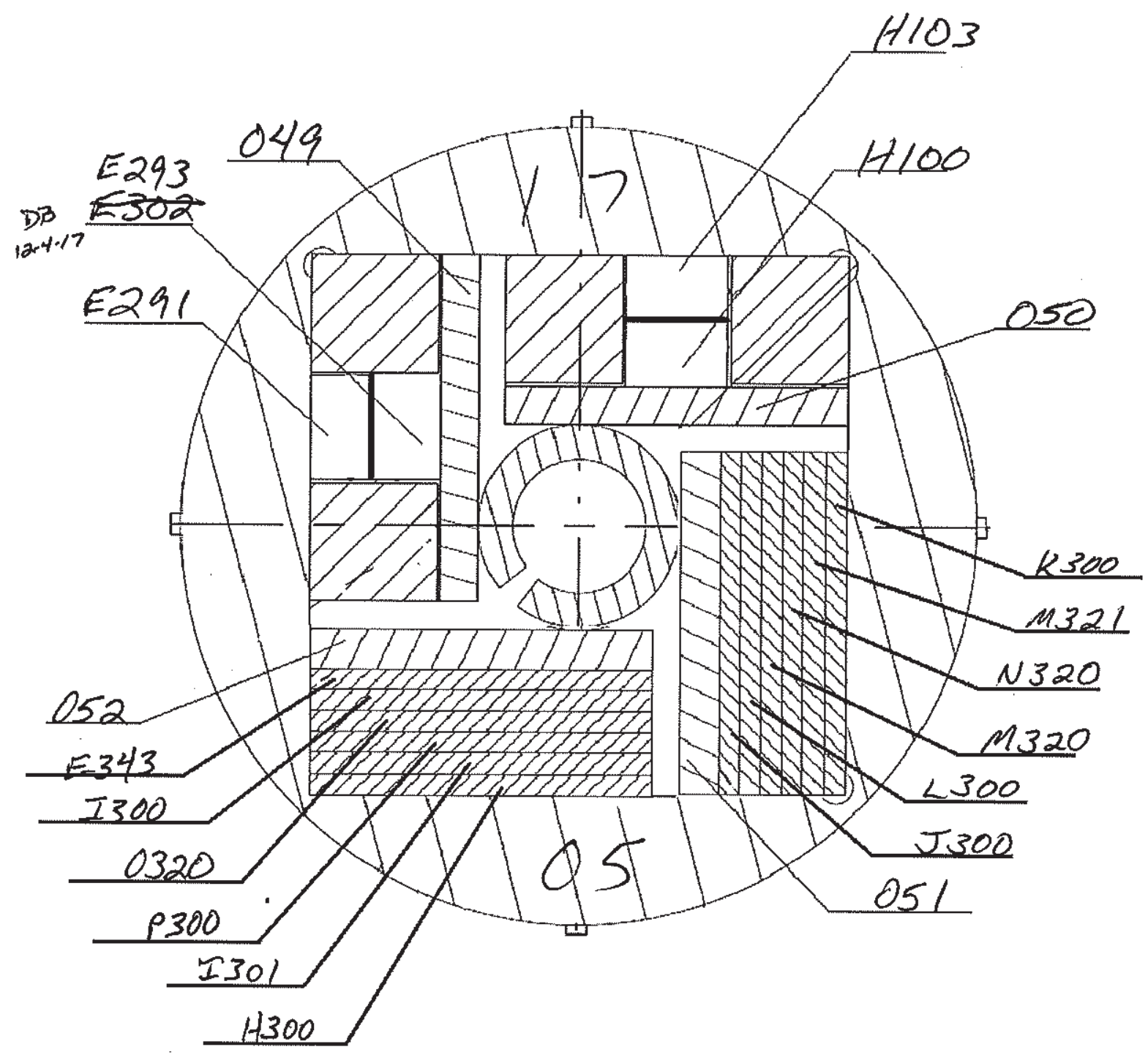


ES21

17-06

(Middle)

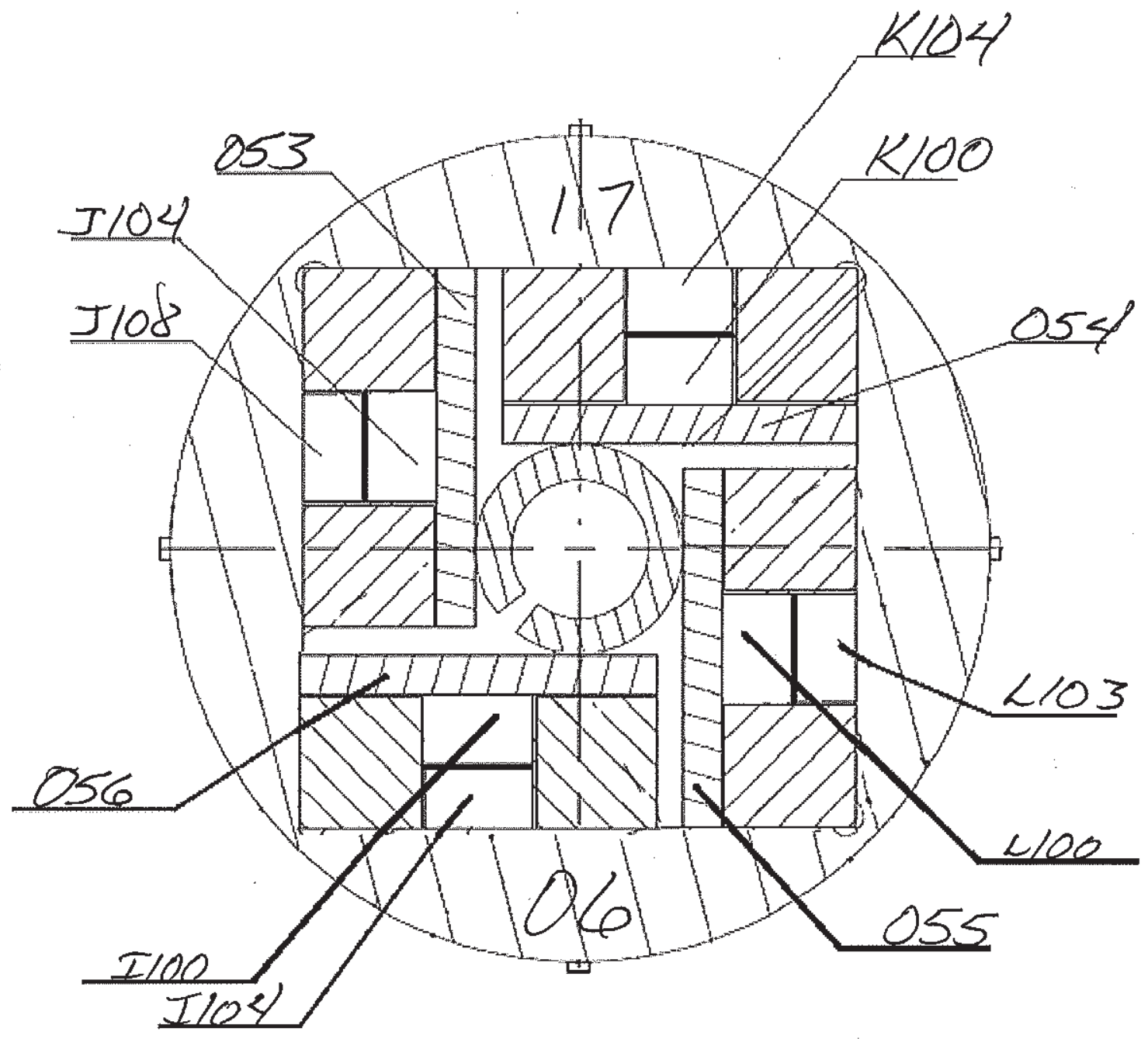




\section{ES21}

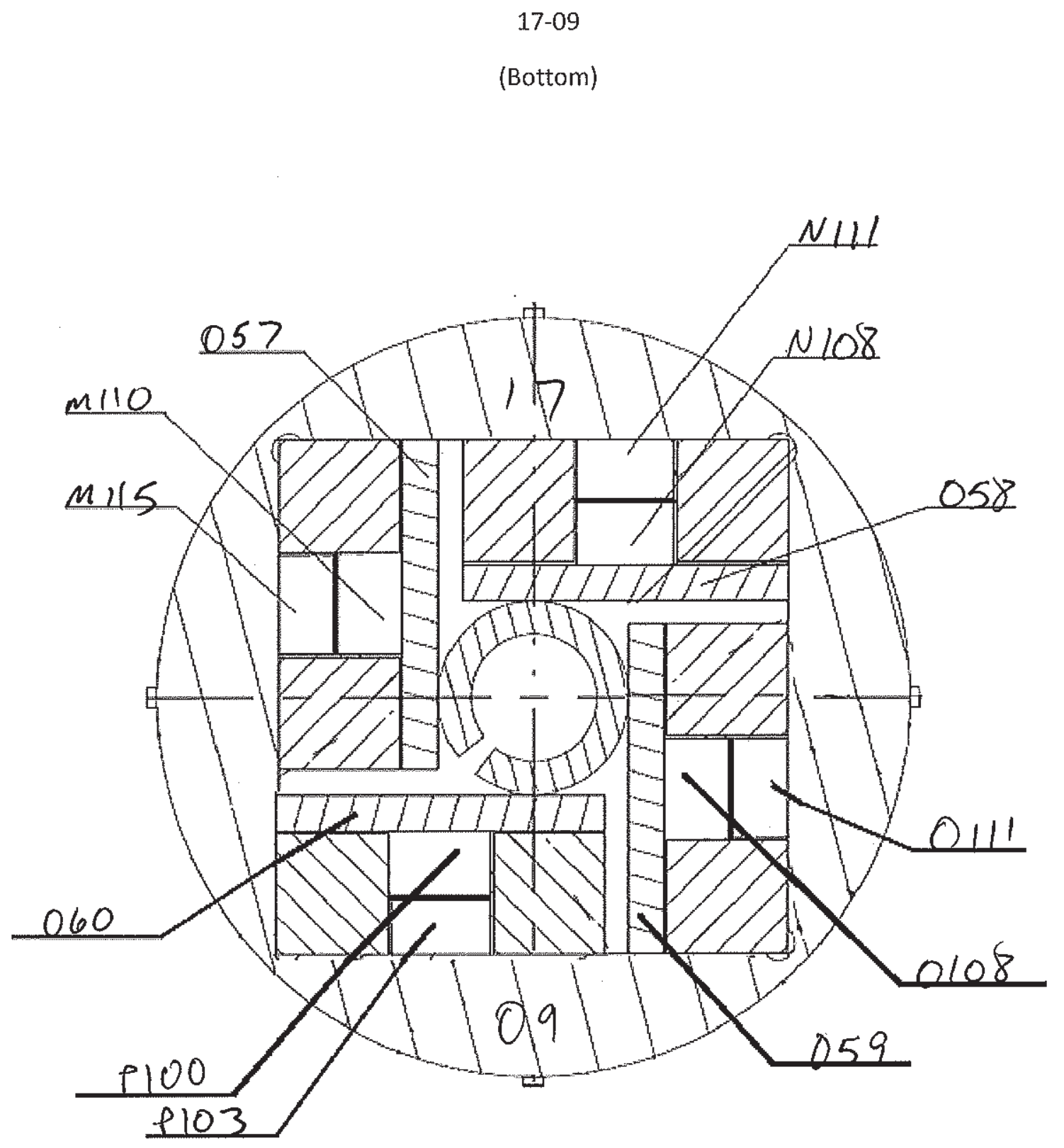




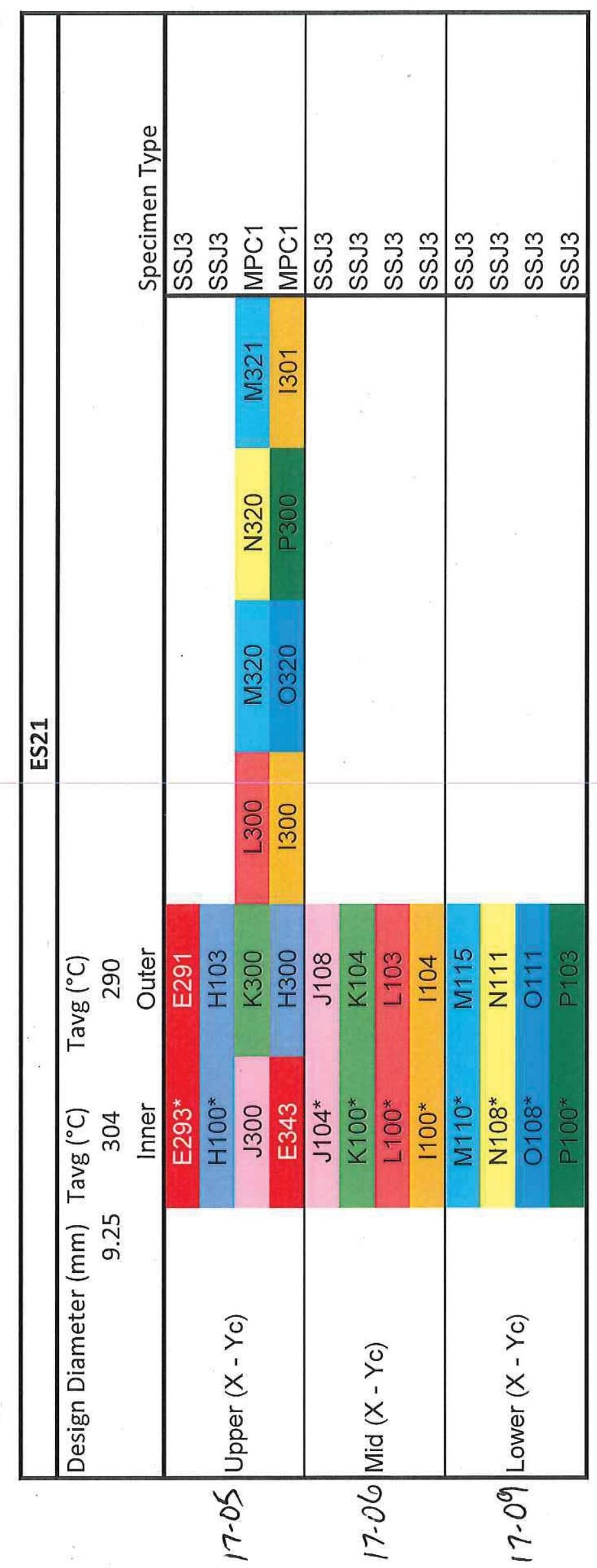




\section{RABBIT ASSEMBLY: ES 21 HOUSING}

\section{ES21}

SN / IR NUMBER:

$$
17-157 / 2 \varnothing 838
$$

INNER

DIAMETER:

$9.53 \mathrm{~mm}$

\section{$\operatorname{HOLDER}(\mathrm{S})$}

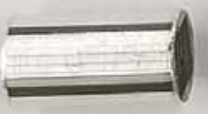

SN / IR NUMBER:

SN / IR NUMBER:

SN / IR NUMBER:

$17-\phi 5 / 2 \not 783$

$17-\not 6 / 20783$

$17-\varnothing 9 / 2 \varnothing 783$

OUTER

DIAMETER:

OUTER

OUTER

DIAMETER:

DIAMETER:

$$
9.24 \mathrm{~mm}
$$

$9.25 \mathrm{~mm}$ 


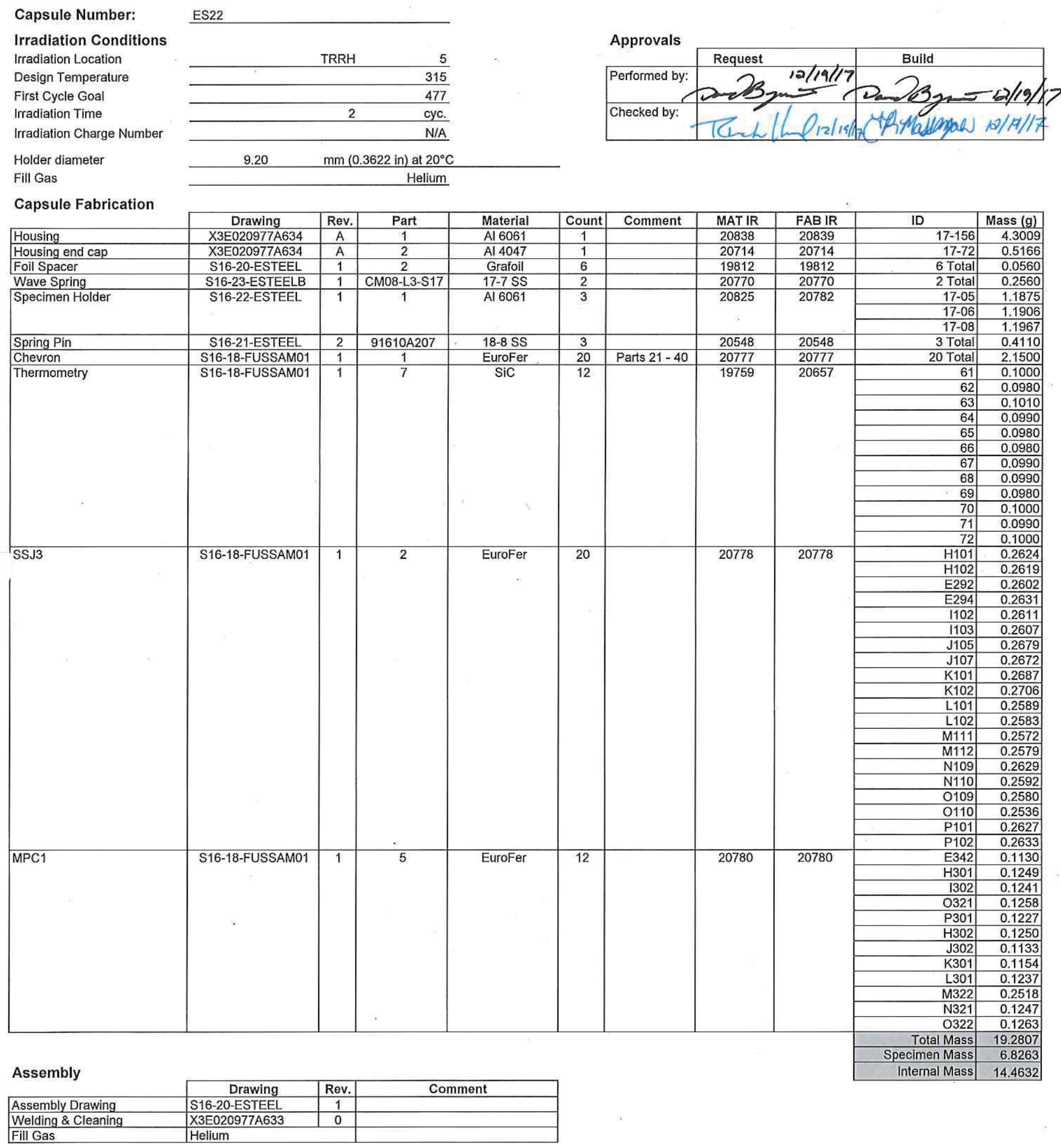


ES22

17-05

(Top)

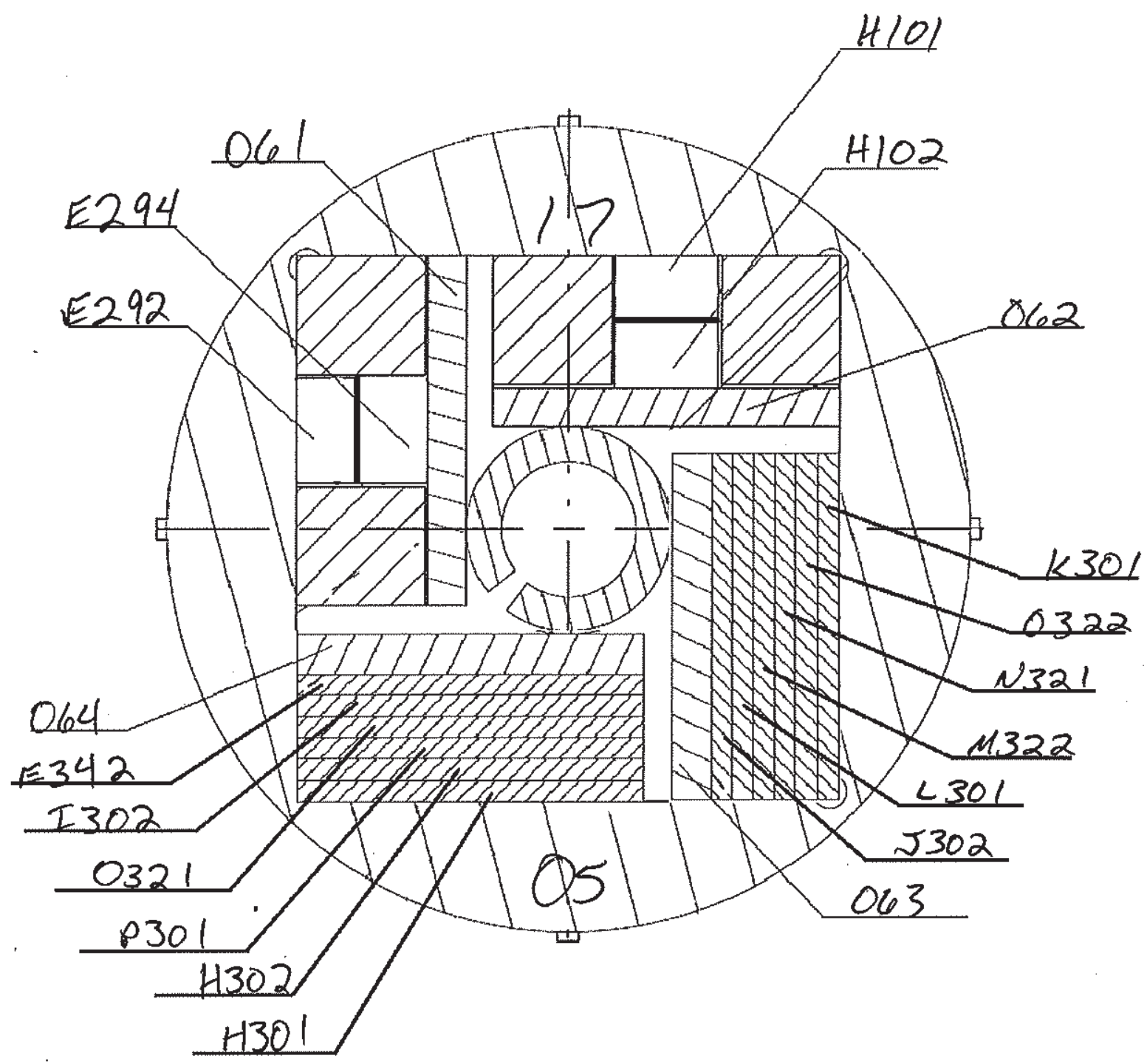


ES22

17-06

(Middle)

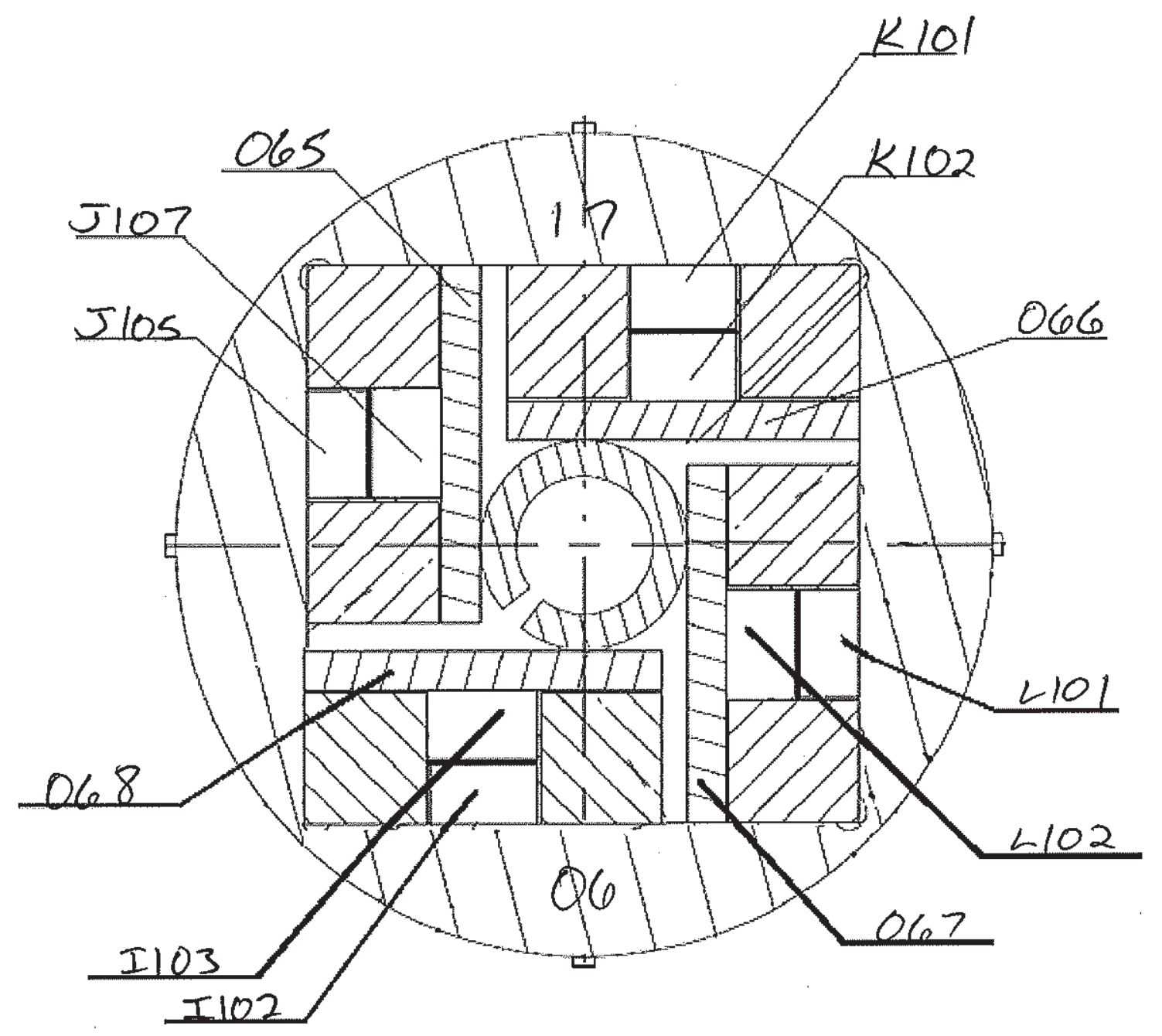




\section{ES22}

\section{7-08}

(Bottom)

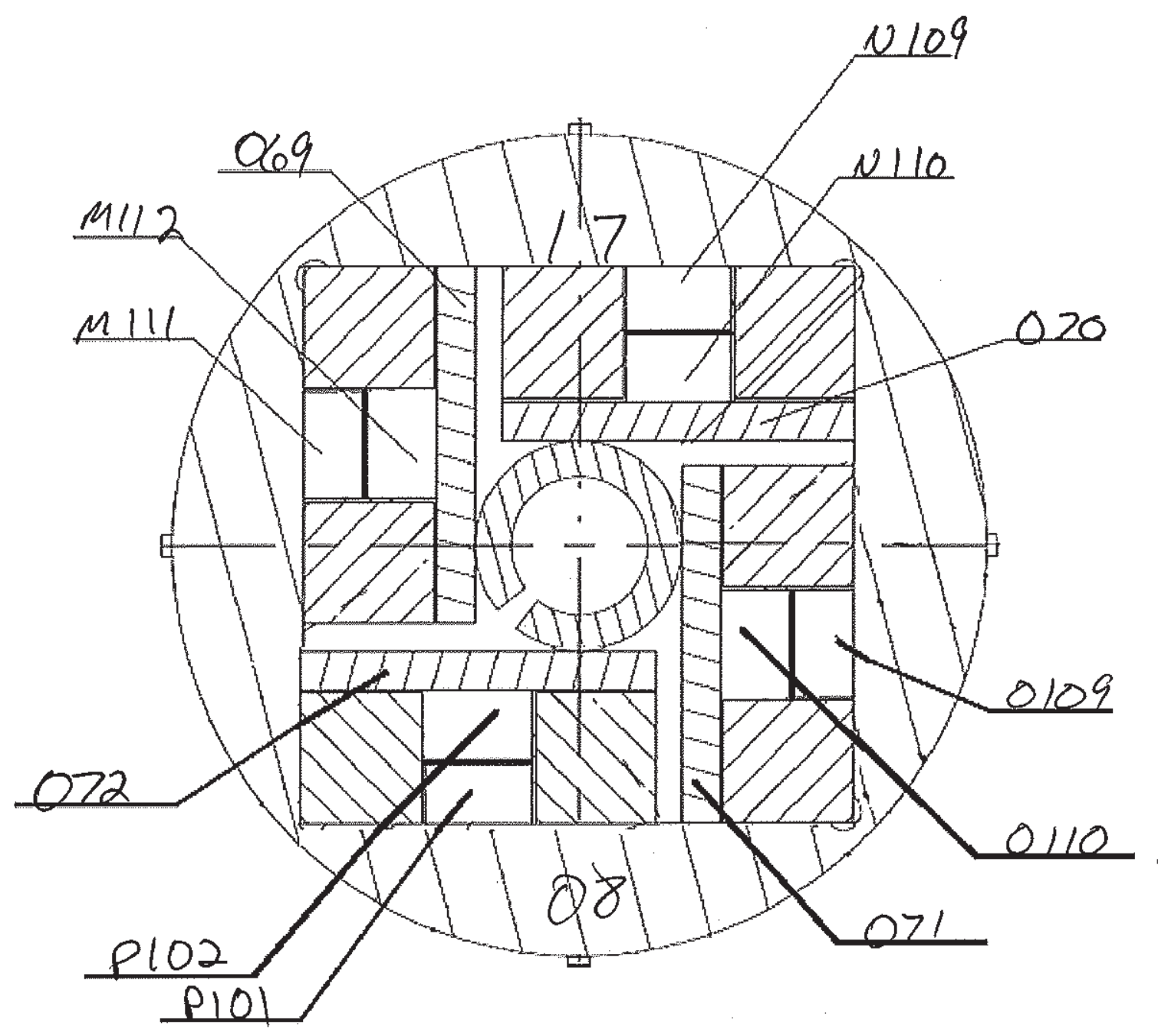




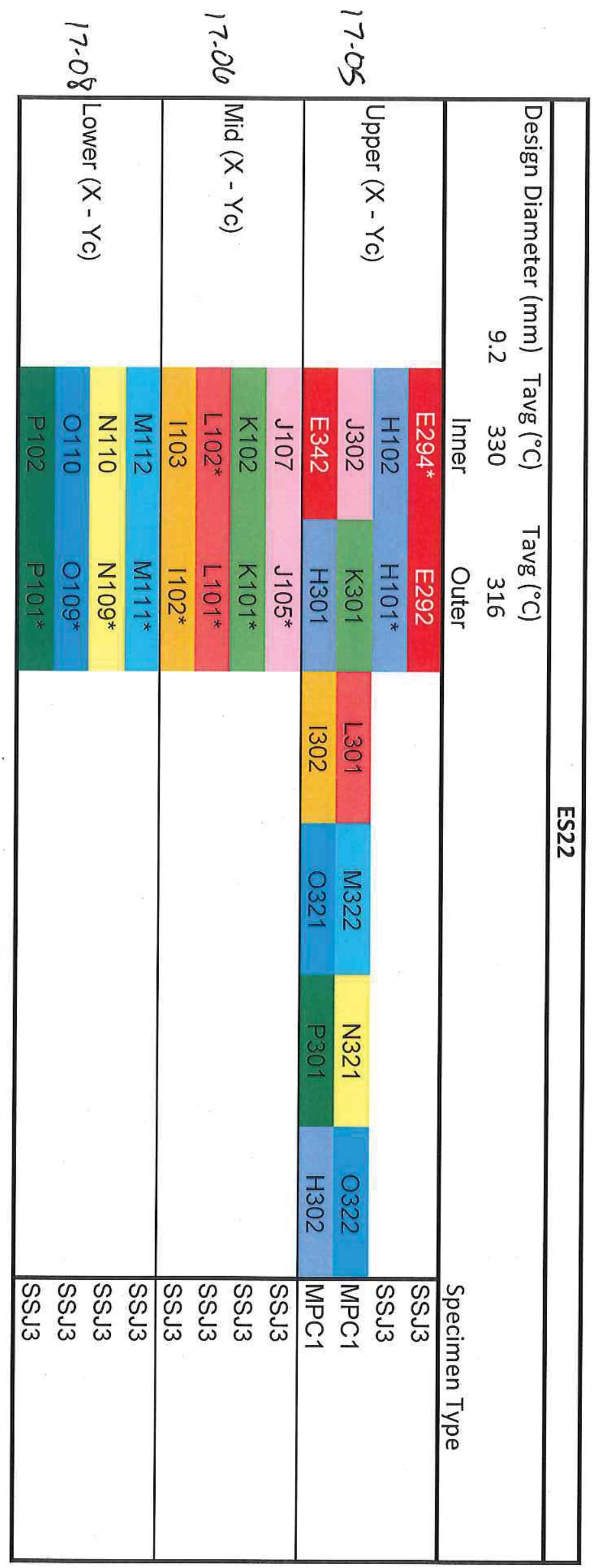




\section{RABBIT ASSEMBLY: ES 22 HOUSING}

\section{ES22}

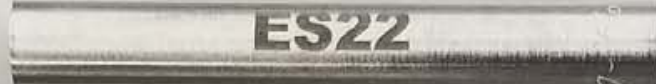

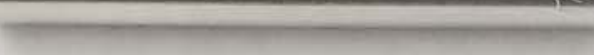

SN / IR NUMBER:

$17-156 / 288838$

INNER

DIAMETER:

$$
\underline{9.51 \mathrm{~mm}}
$$

\section{HOLDER(S)}

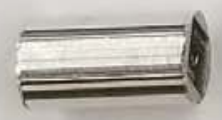

SN / IR NUMBER:

SN / IR NUMBER:

$17-\$ 55 / 2 \varnothing 782$

$$
17-\not 66 / 28782
$$

DIAMETER:
OUTER

q. $19 \mathrm{~mm}$

OUTER

DIAMETER:

q. $18 \mathrm{~mm}$

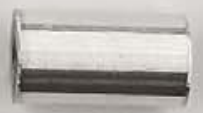

SN / IR NUMBER:

$17-\varnothing 8 / 28782$

OUTER

DIAMETER:

$9.2 \phi_{\mathrm{mm}}$ 


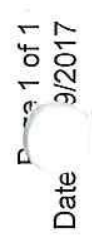

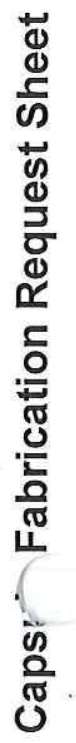
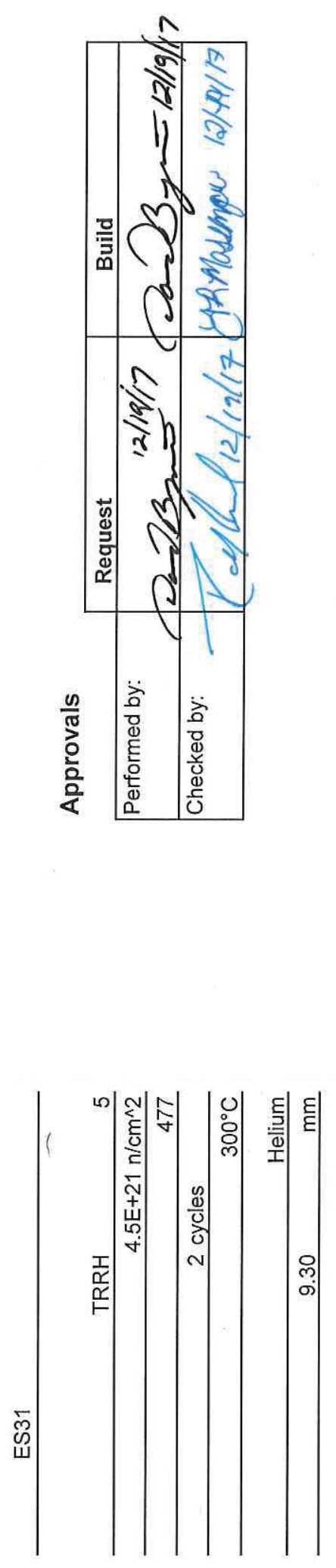
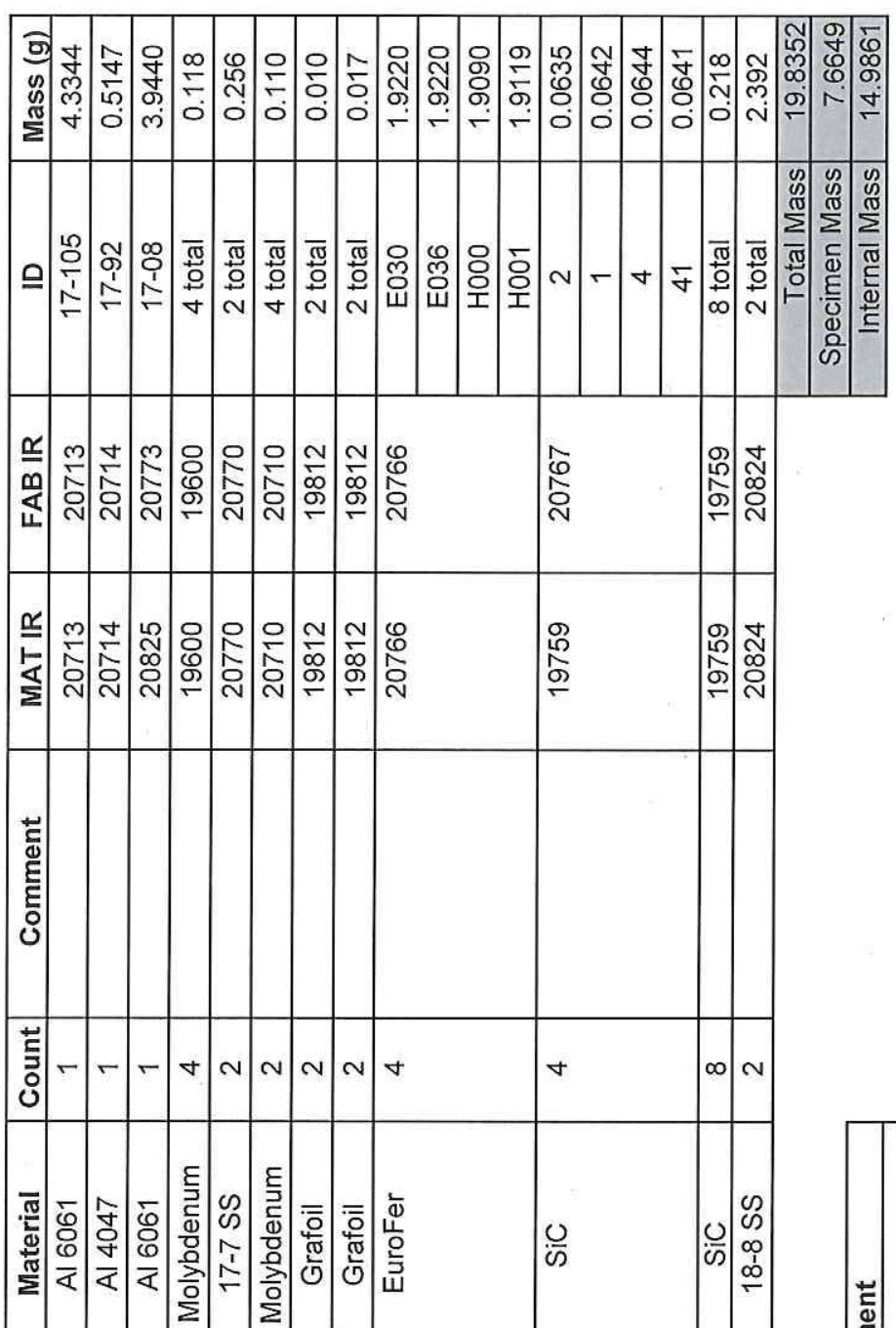
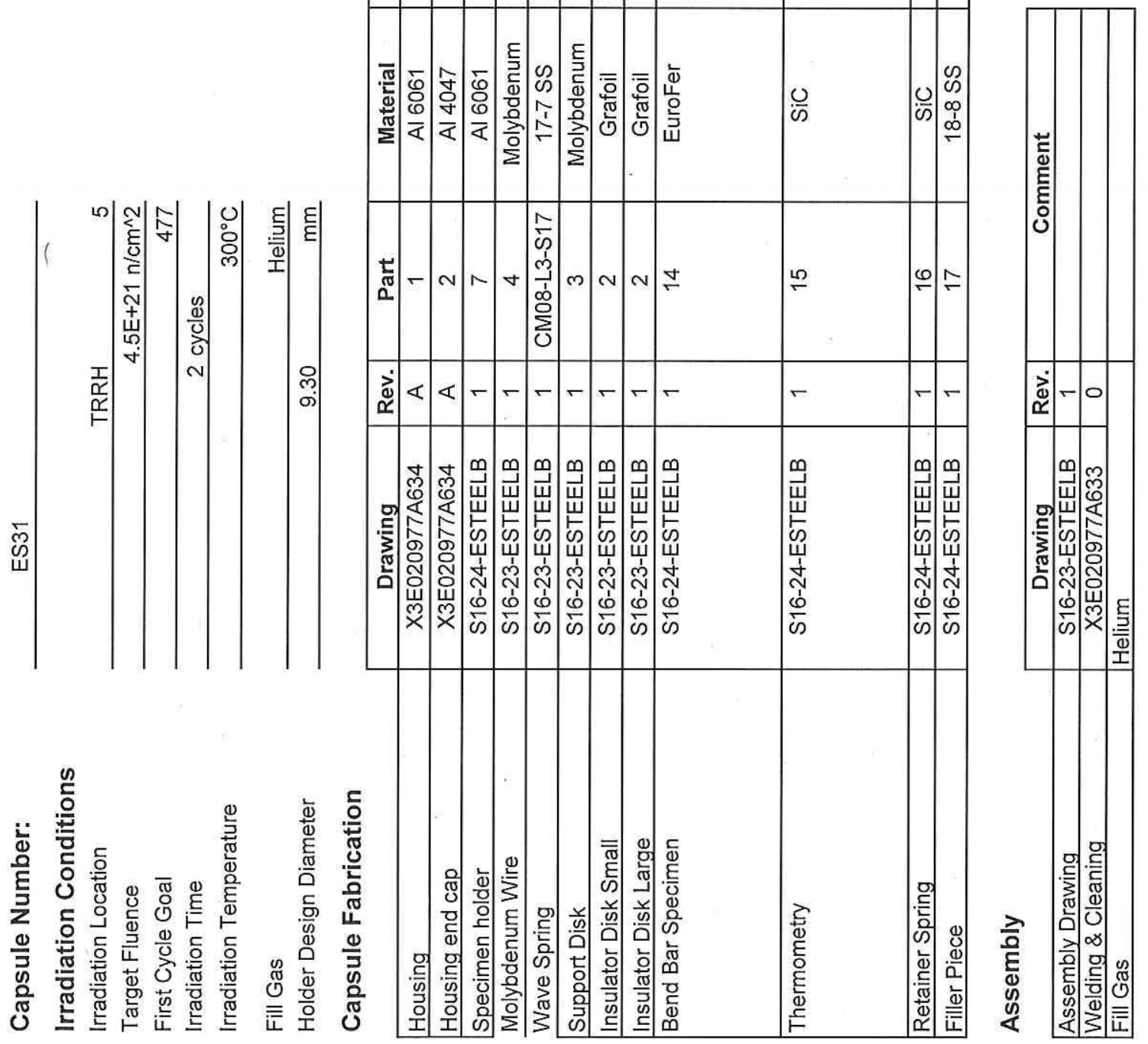
ES31

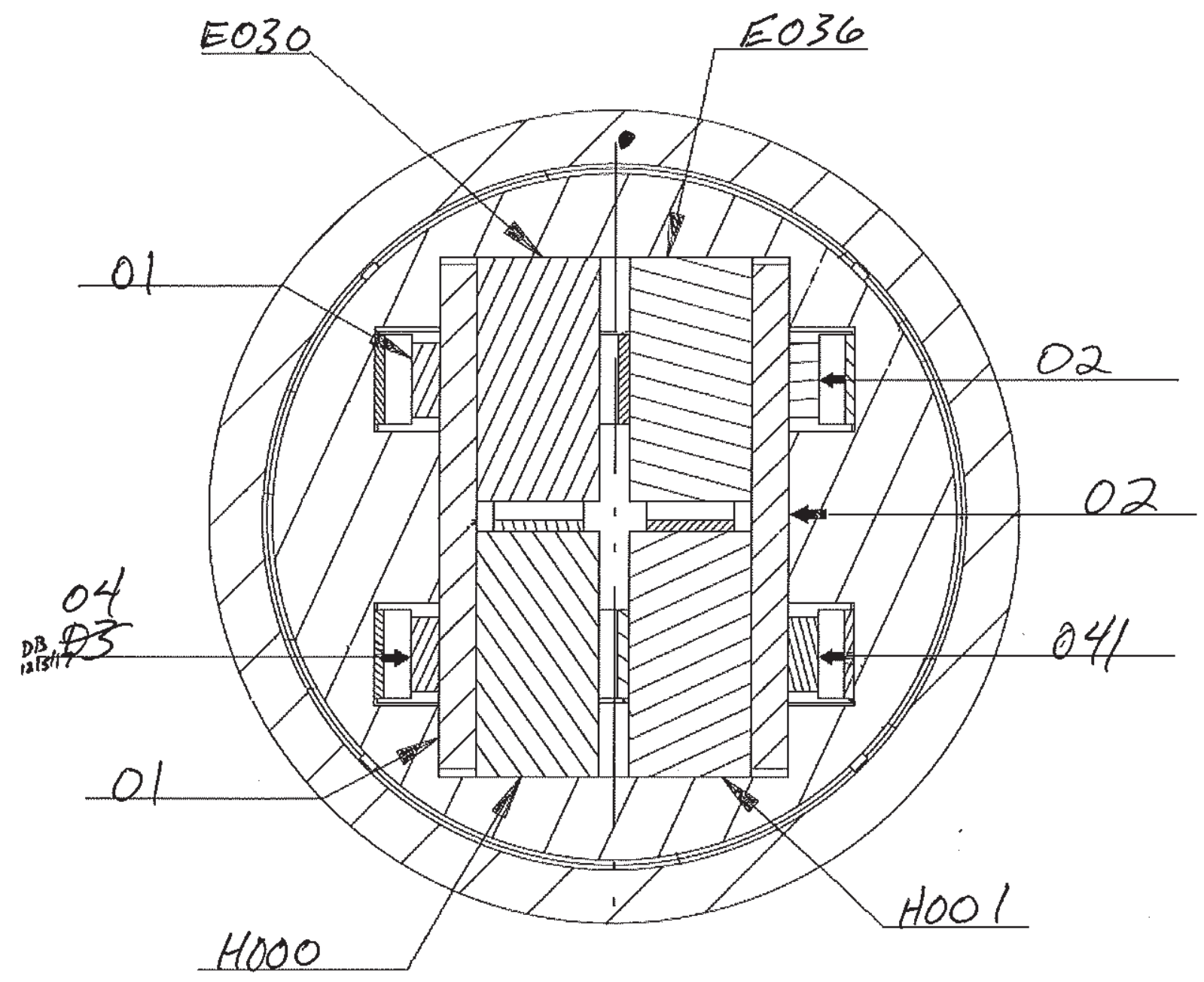




\section{RABBIT ASSEMBLY: ES3] HOUSING}

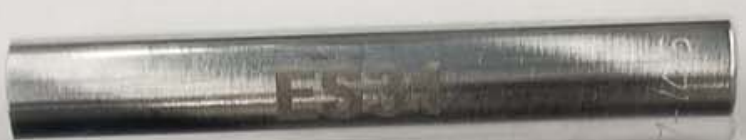

SN / IR NUMBER:

$17-1 \phi 5 / 2 \phi 713$

INNER

DIAMETER:

$9.53 \mathrm{~mm}$

$\operatorname{HOLDER}(\mathrm{S})$

SN / IR NUMBER:

$17-\varnothing 8 / 28773$

OUTER DIAMETER: 


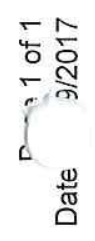

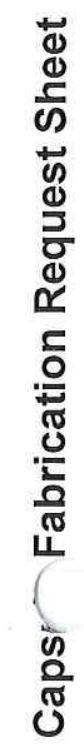

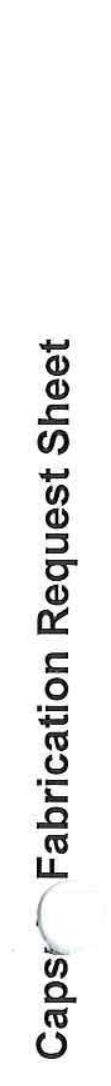

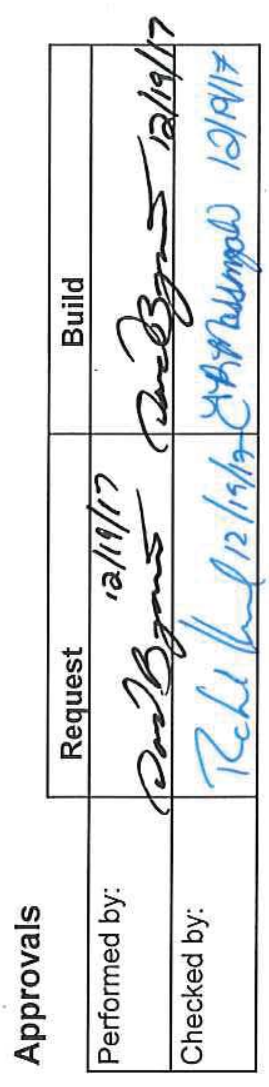

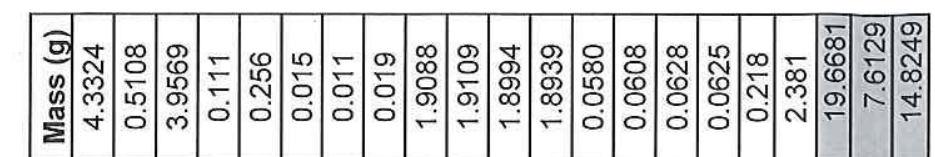

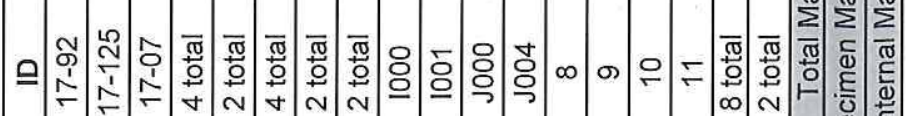

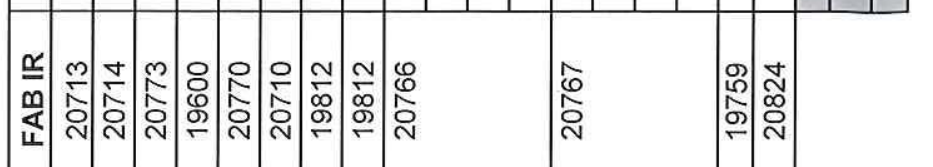

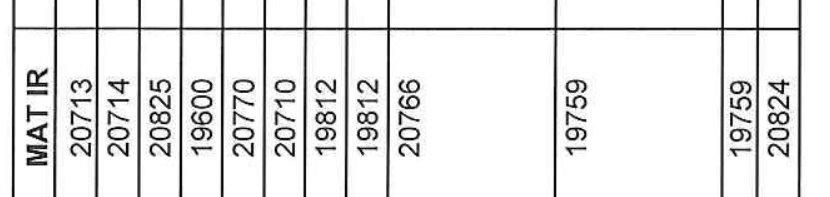
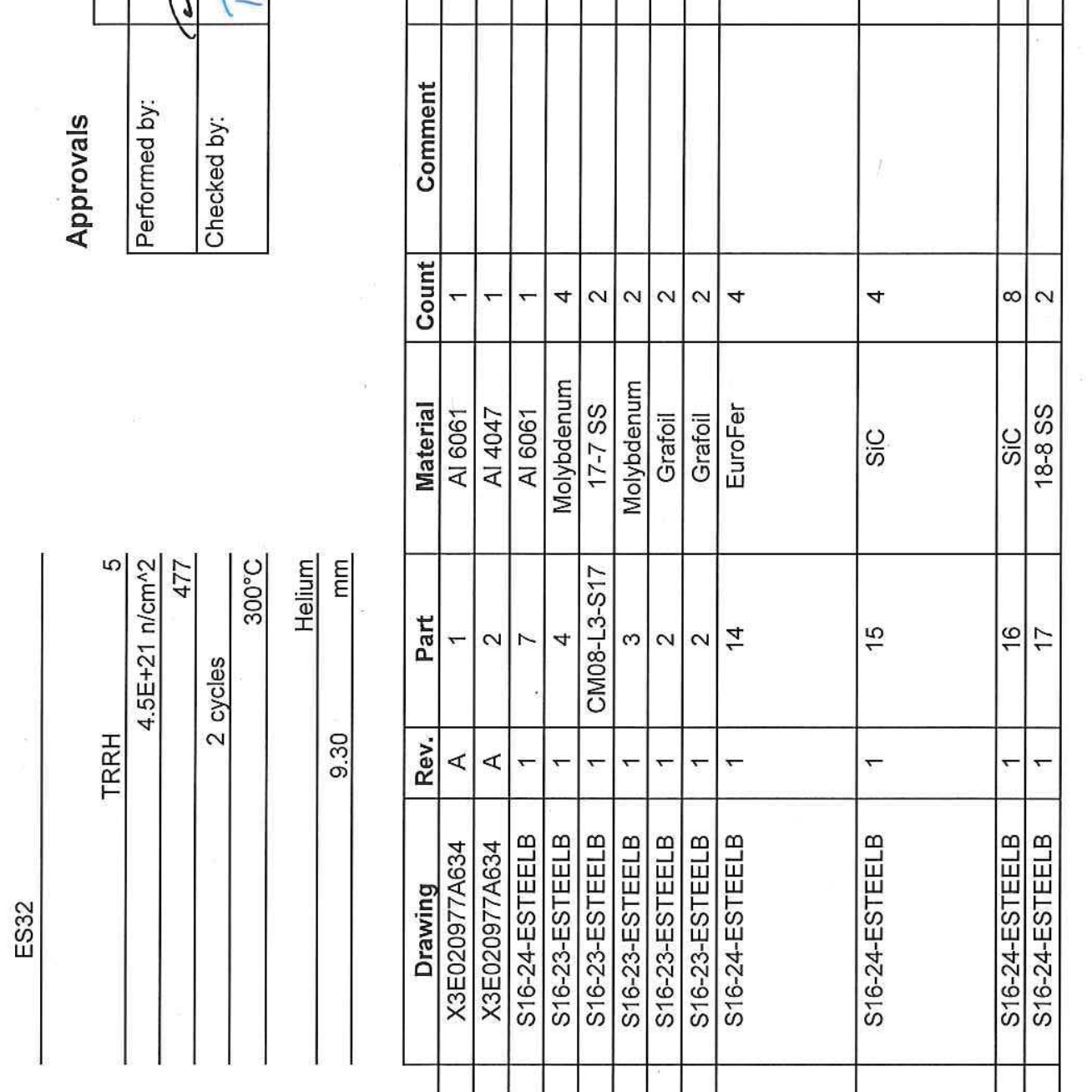
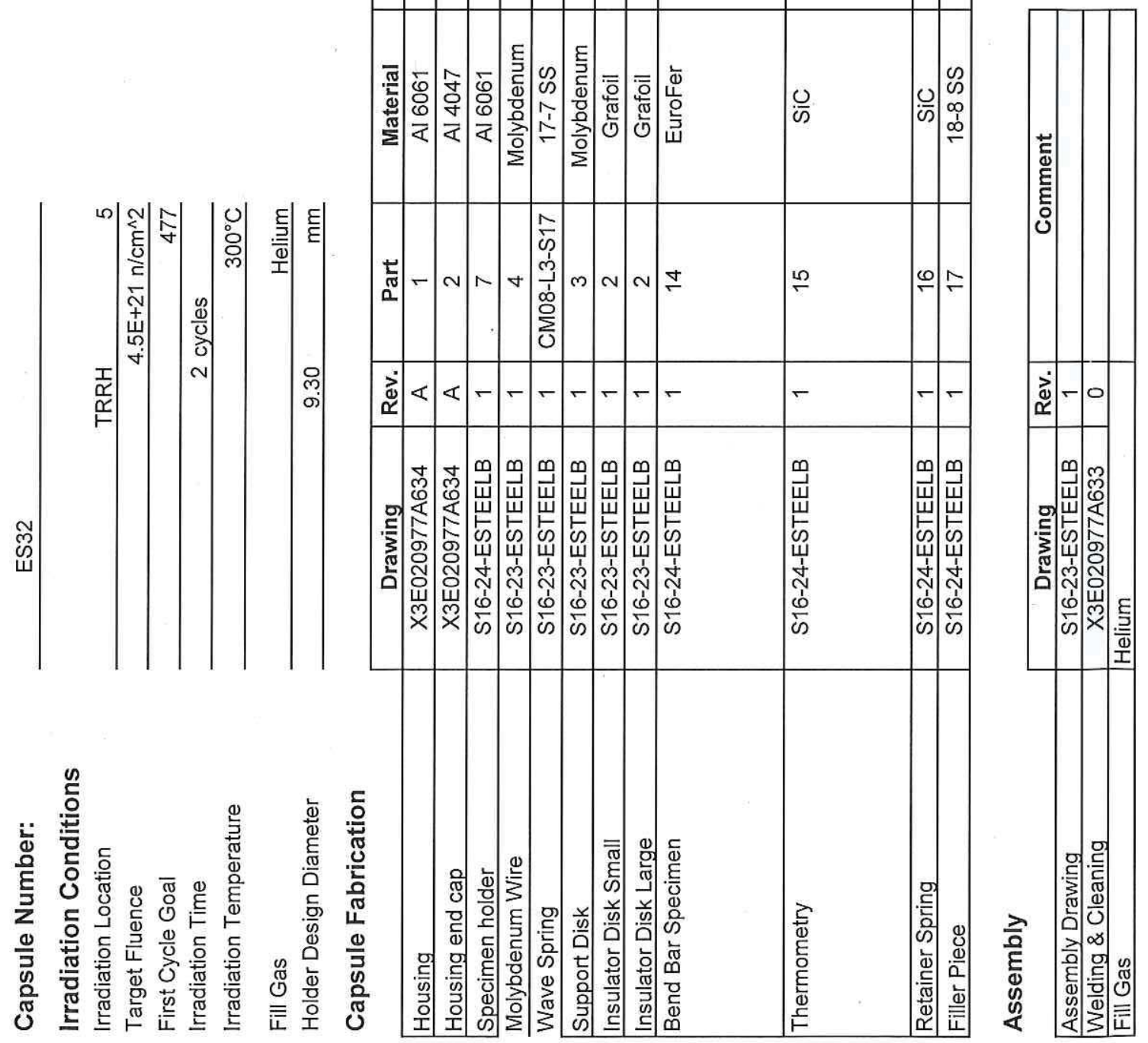
ES 32

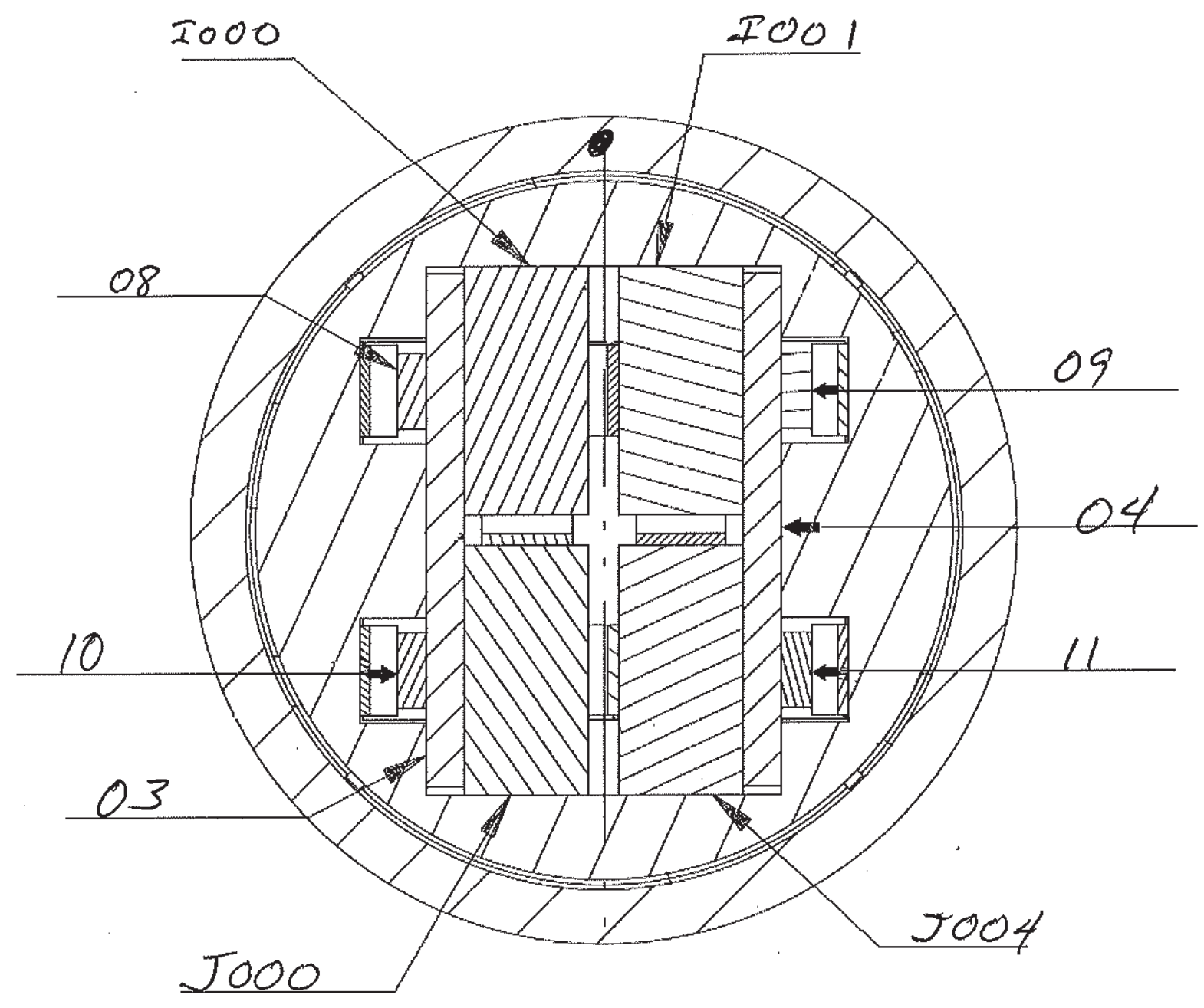




\section{RABBIT ASSEMBLY: ES 32}

\section{HOUSING}

\section{- 432}

SN / IR NUMBER:

$17-92 / 2 \phi 713$

INNER

DIAMETER:

$9.52 \mathrm{~mm}$

HOLDER(S)

SN / IR NUMBER:

$17-\varnothing 7 / 2 \varnothing 773$

OUTER DIAMETER:

9. $3 \varnothing \mathrm{mm}$ 
$\frac{5}{\frac{5}{0}} \frac{\bar{c}}{\sigma}$

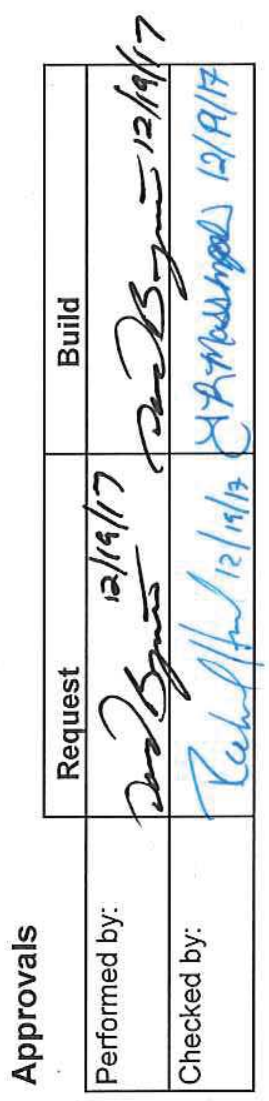

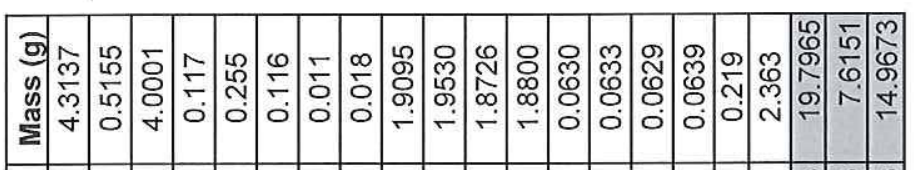

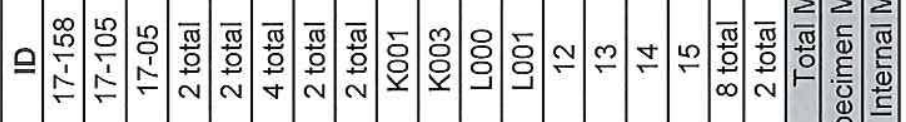

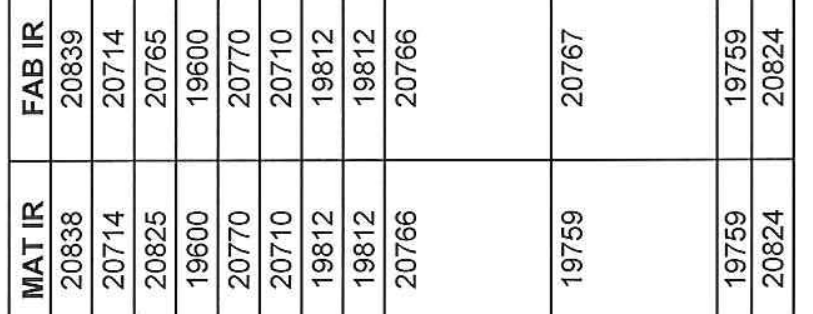

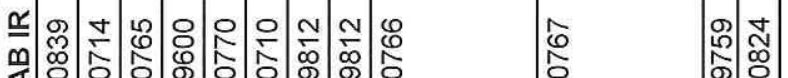

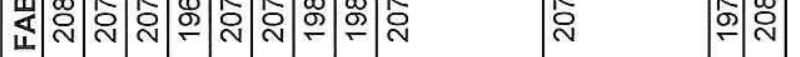

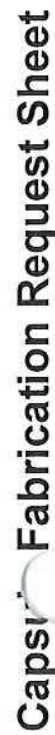

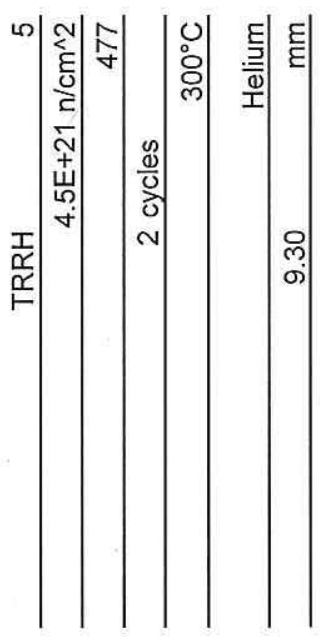

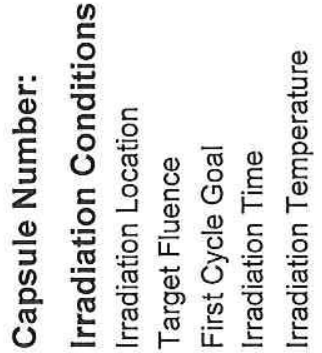

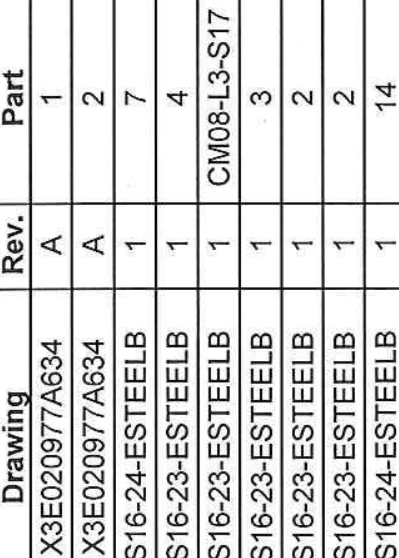

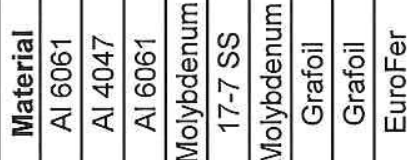
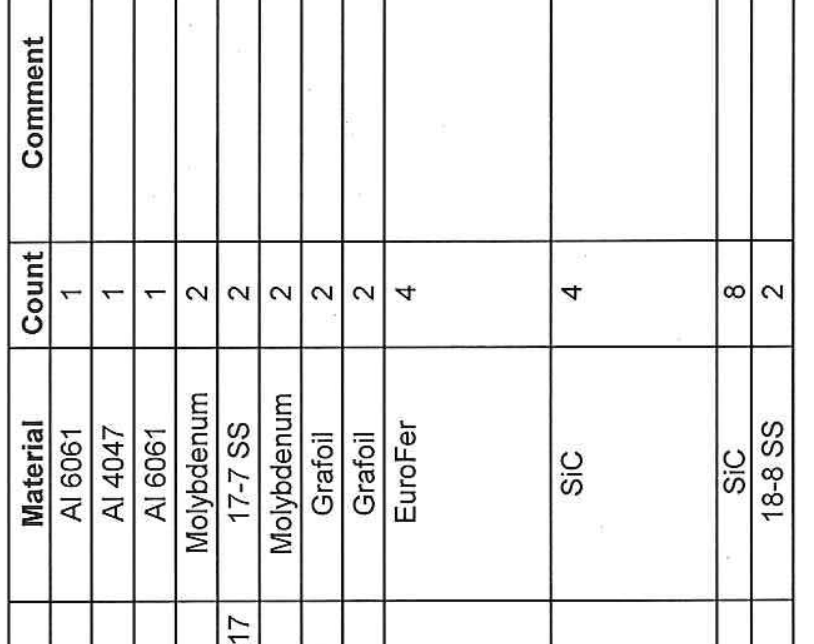

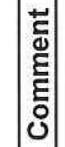

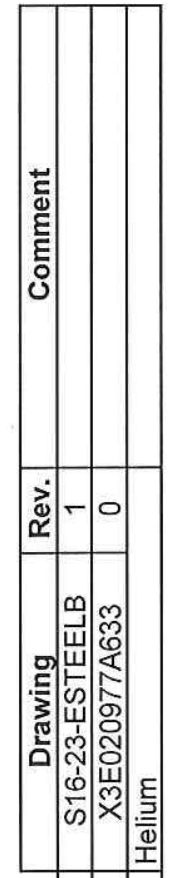




$$
5533
$$

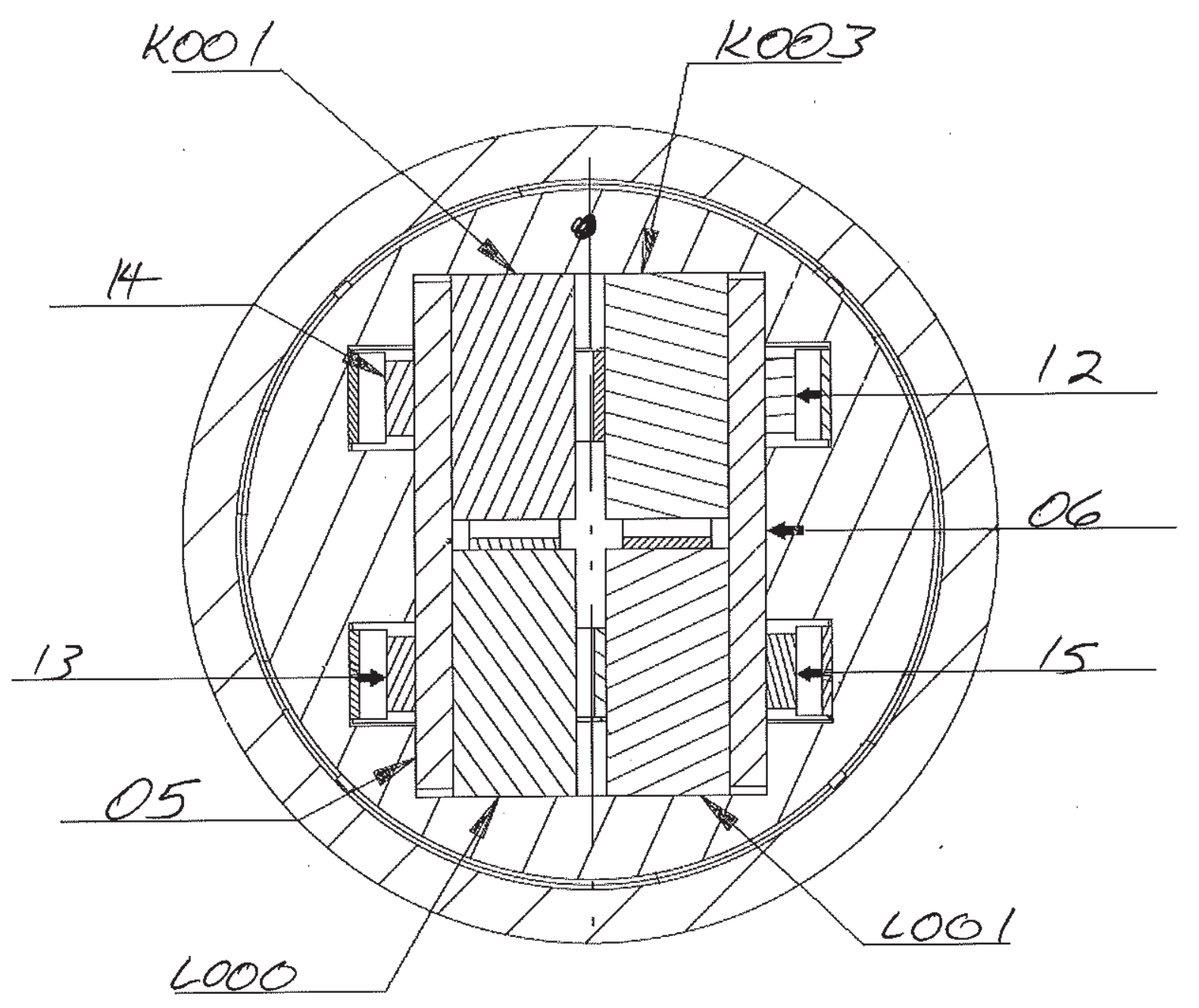




\section{RABBIT ASSEMBLY: ES 33}

\section{HOUSING}

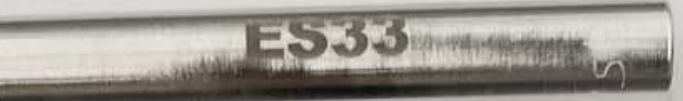

SN / IR NUMBER:

$17-158 / 2 \varnothing 838$

INNER

DIAMETER:

$$
9.53 \mathrm{~mm}
$$

\section{HOLDER(S)}

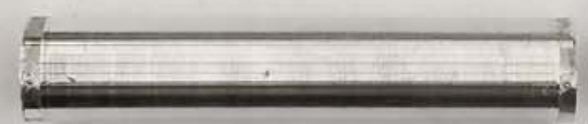

SN / IR NUMBER:

$17-\phi 5 / 2 \varnothing 773$

OUTER

DIAMETER:

9.32 
एँ.

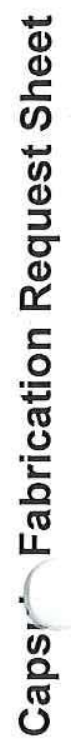
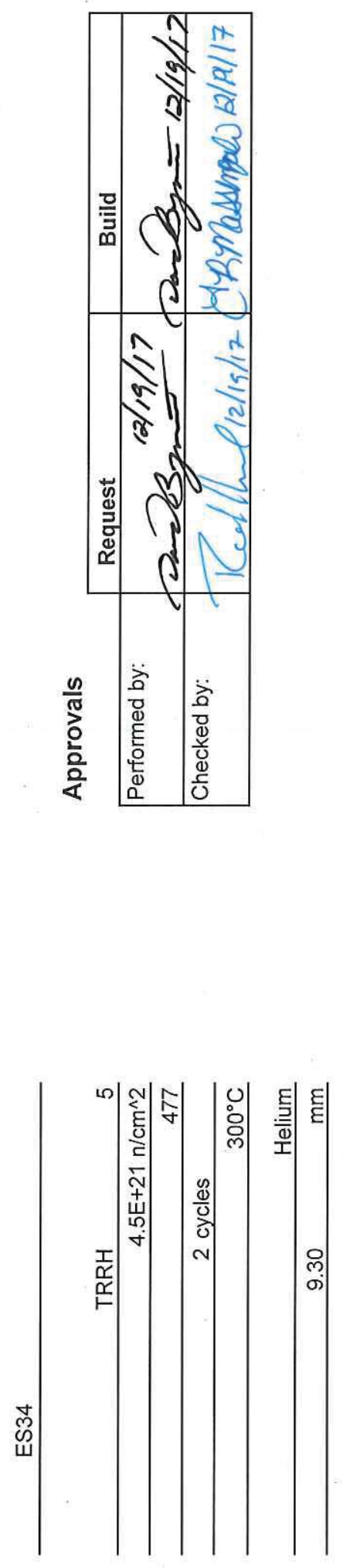

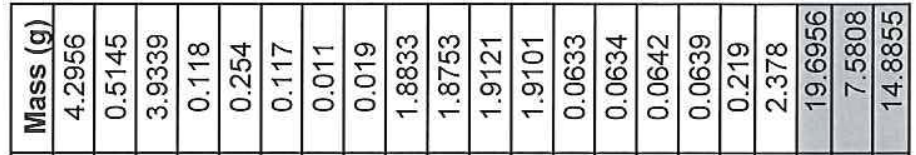

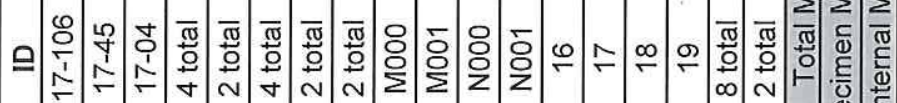
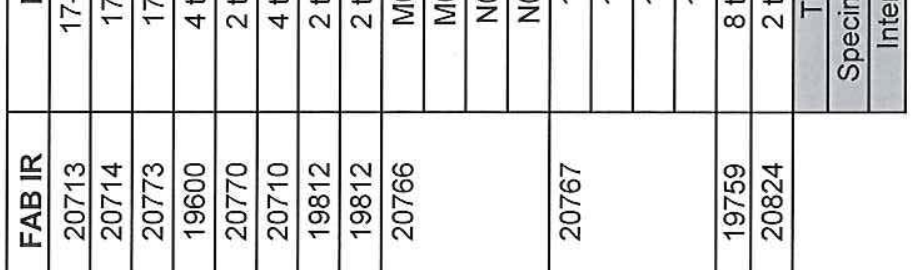

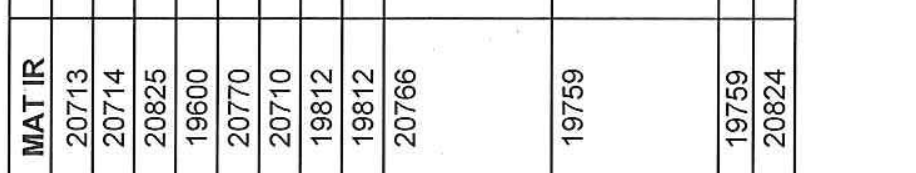

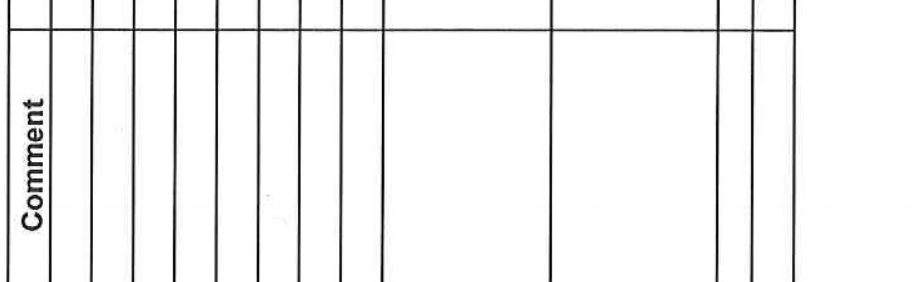

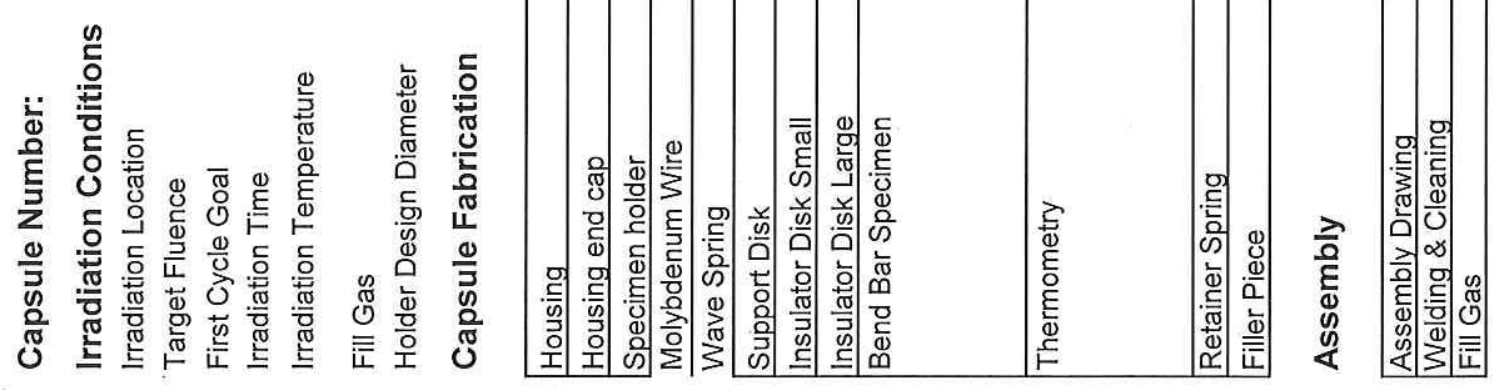




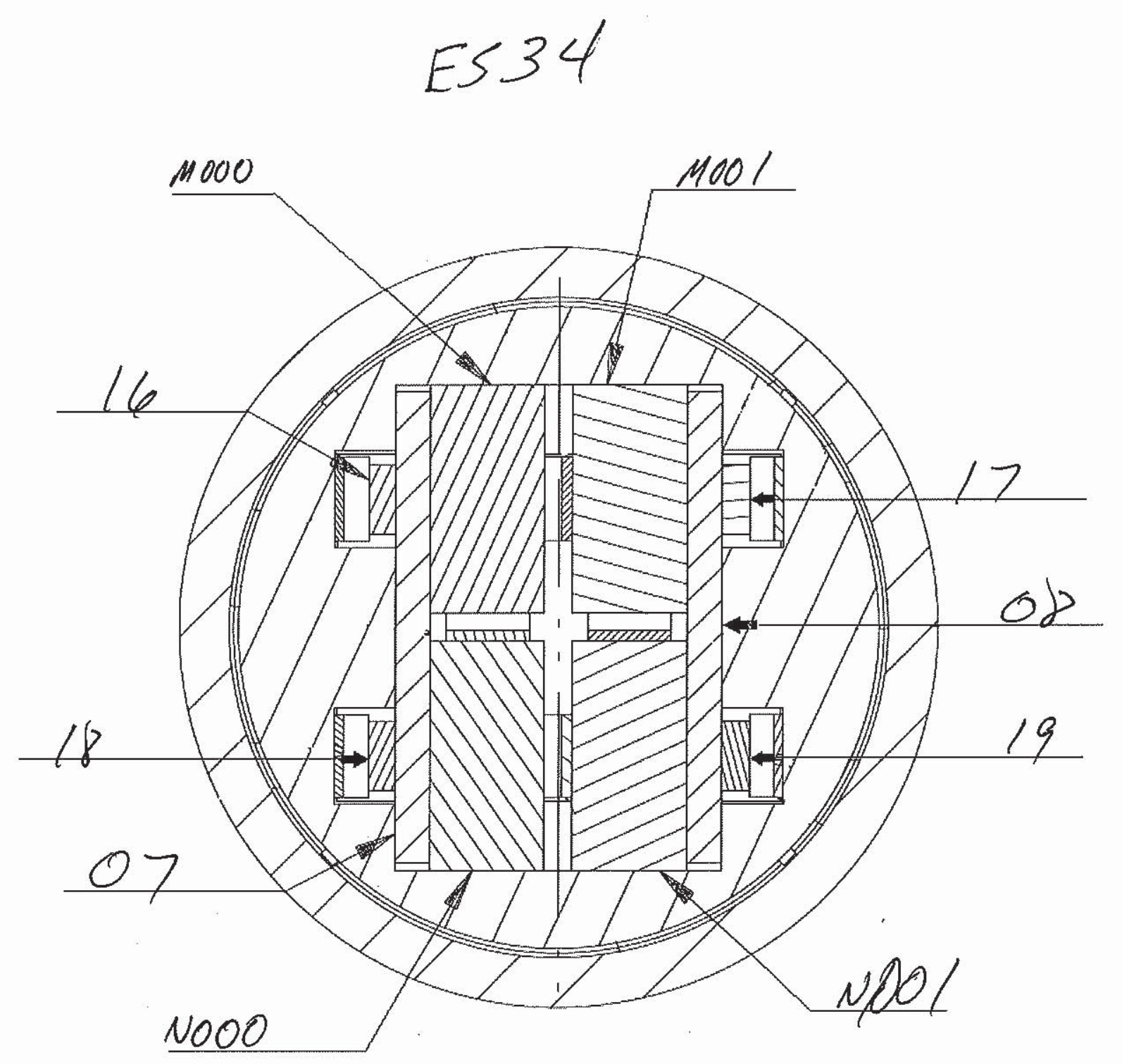




\section{RABBIT ASSEMBLY: ES 34 HOUSING}

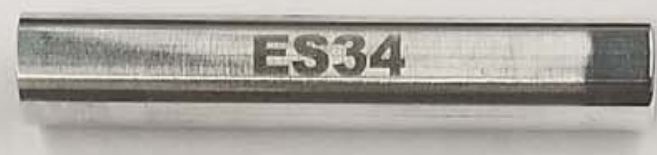

SN / IR NUMBER:

$17-106 / 2 \varnothing 713$

INNER

DIAMETER:

$9.52 \mathrm{~mm}$

\section{$\operatorname{HOLDER}(\mathrm{S})$}

SN / IR NUMBER:

$17-\phi 4 / 2 \varnothing 773$

OUTER

DIAMETER:

q. $29 \mathrm{~mm}$ 
产

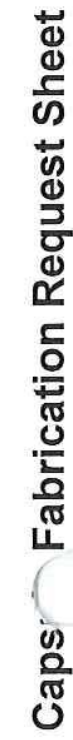
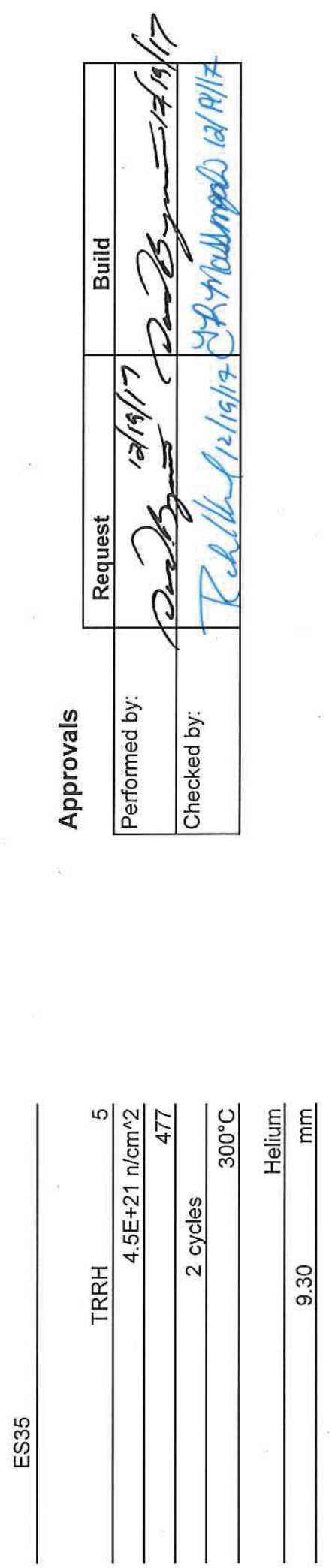

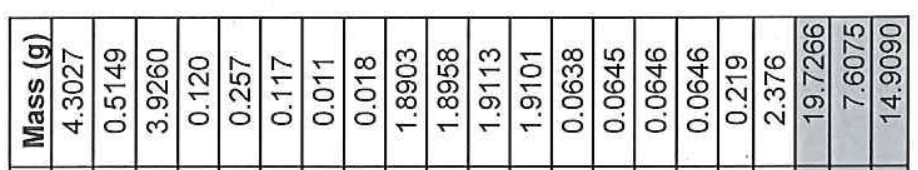

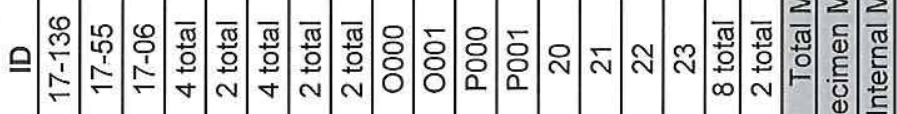

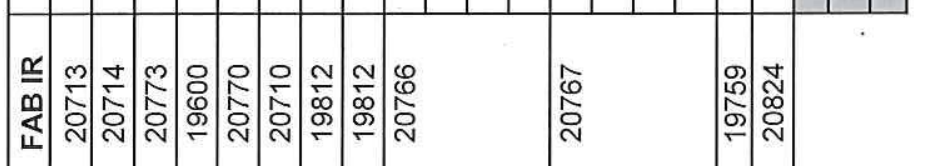

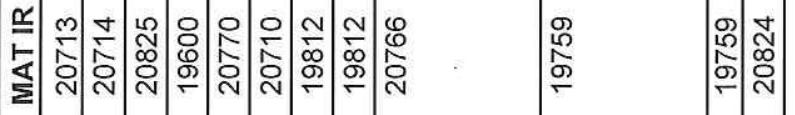
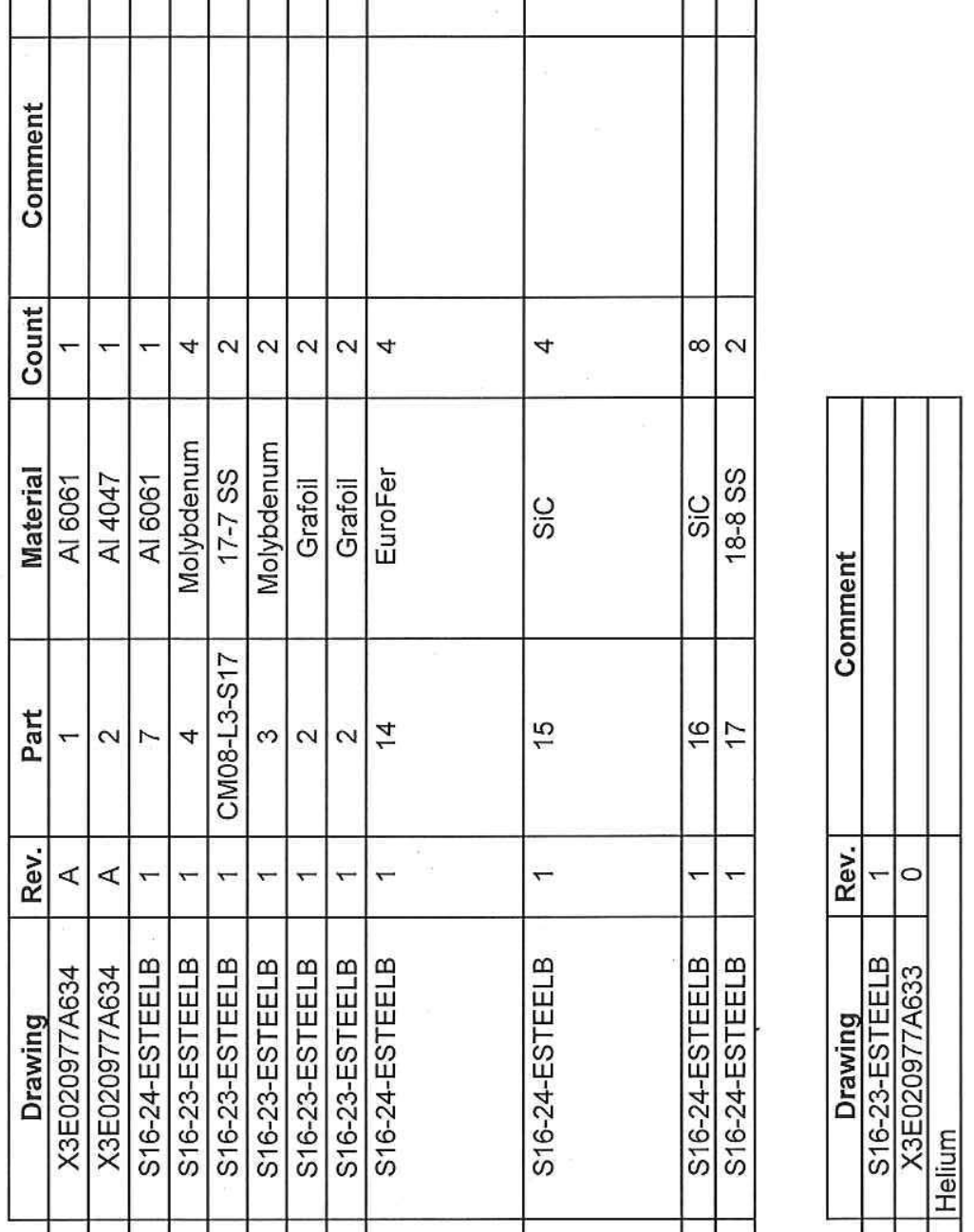

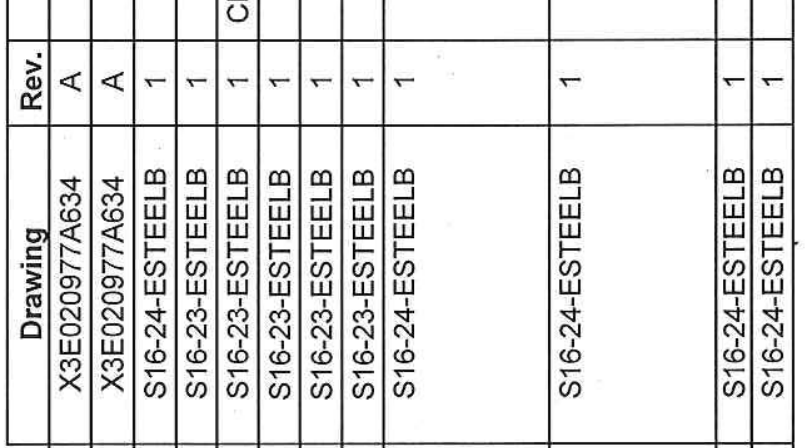
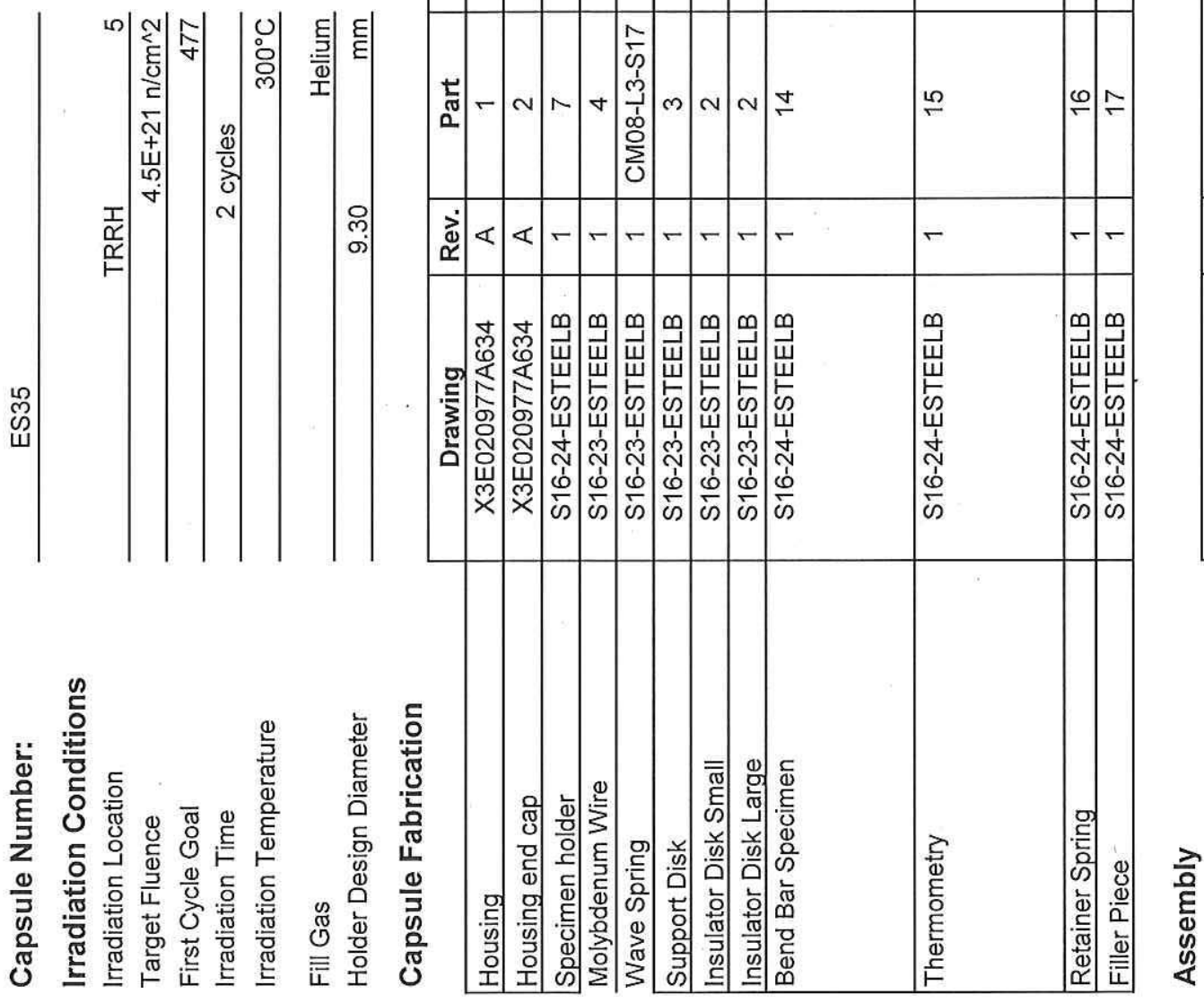

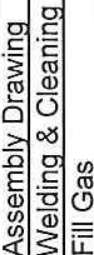




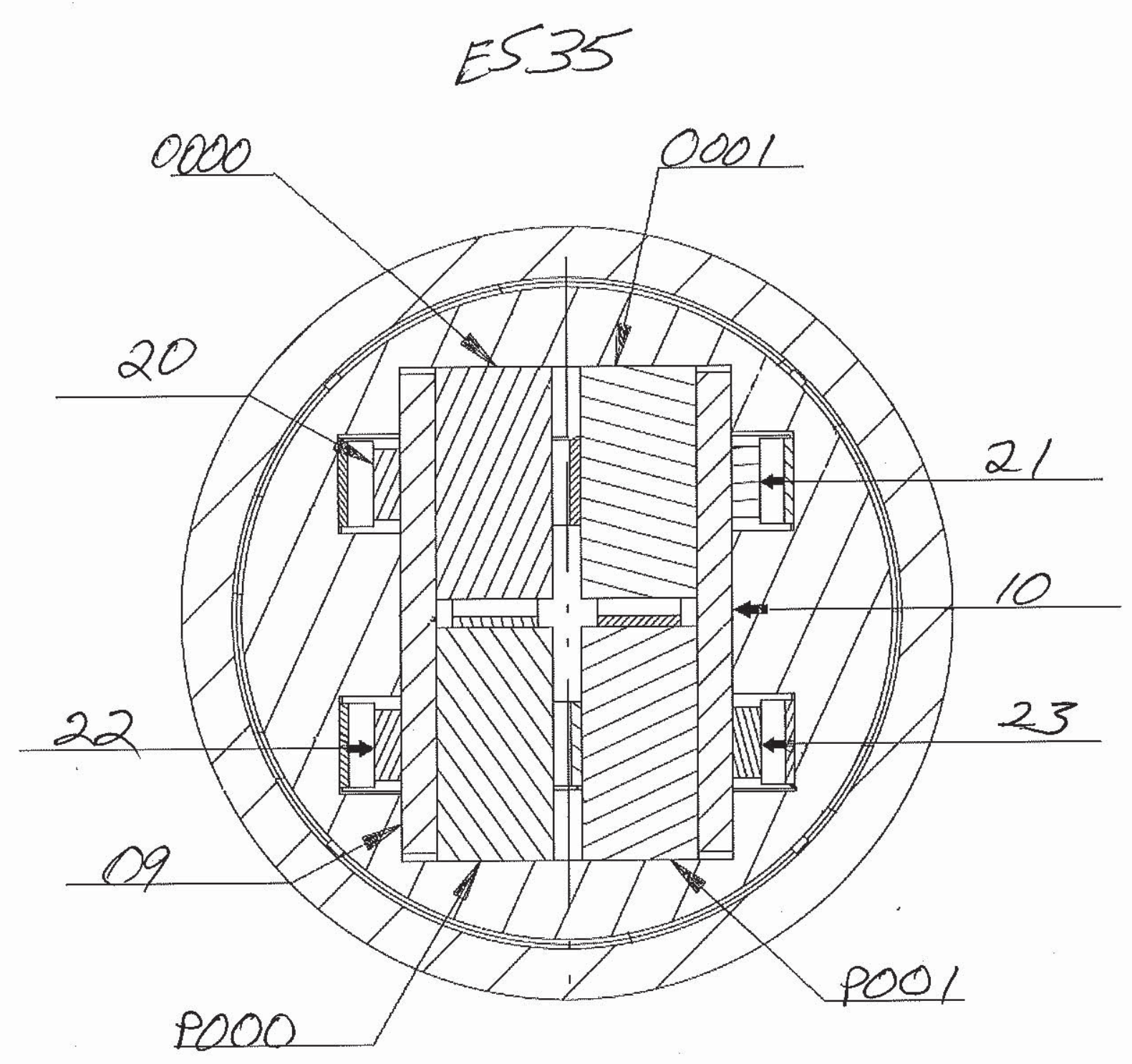


RABBIT ASSEMBLY: ES 35

HOUSING

ES35

SN / IR NUMBER:

$17-13 \underline{6} / 2 \varnothing 713$

INNER

DIAMETER:

$9.53 \mathrm{~mm}$

$\operatorname{HOLDER}(\mathrm{S})$

SN / IR NUMBER:

$17-\not 55 / 2 \not 765$

OUTER

DIAMETER:

$9.3 \varnothing \mathrm{mm}$ 